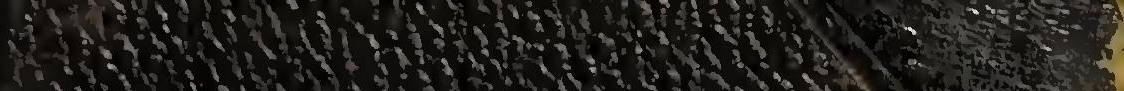

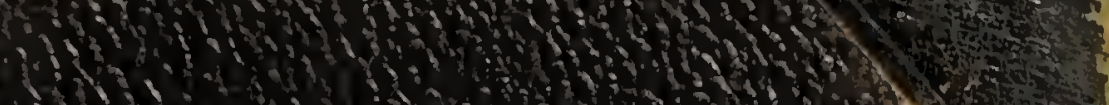

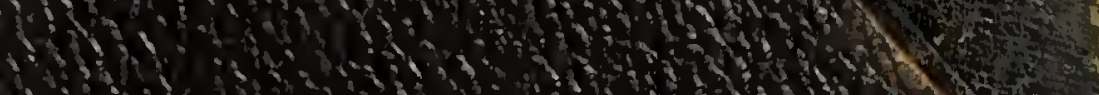

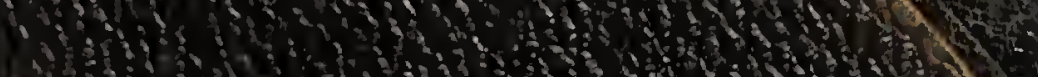

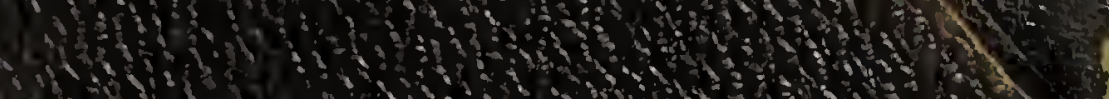
14.

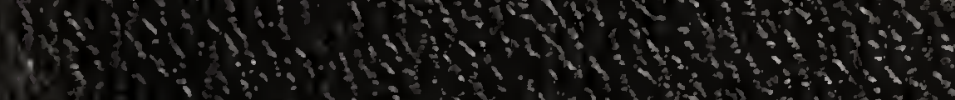

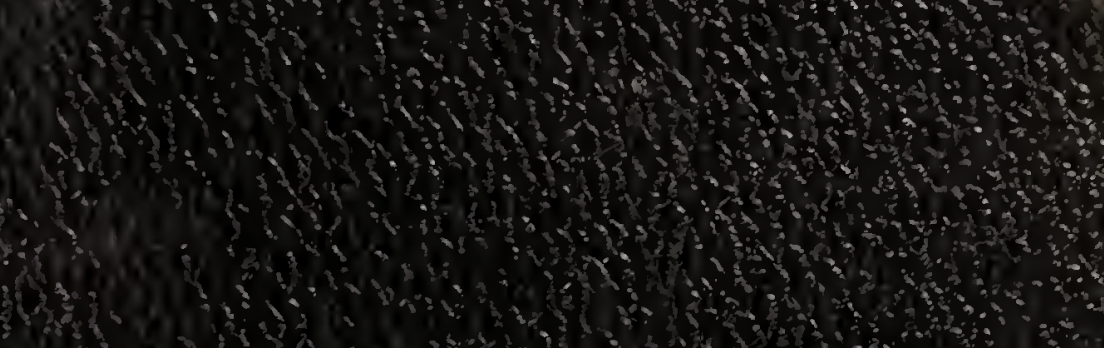

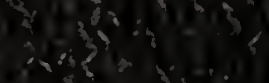

(5)

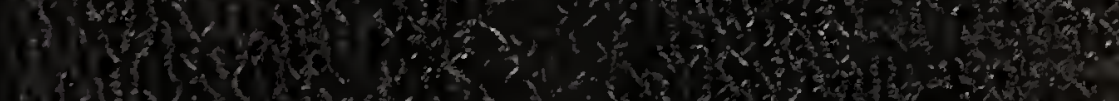
13 है

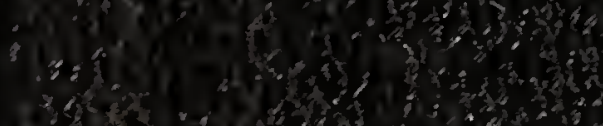

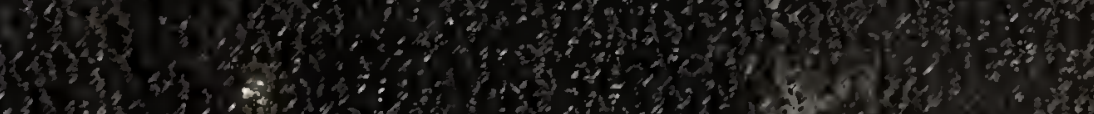

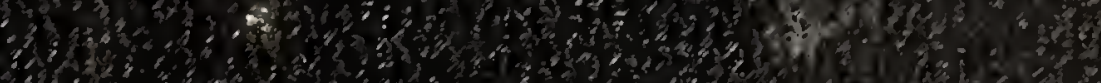

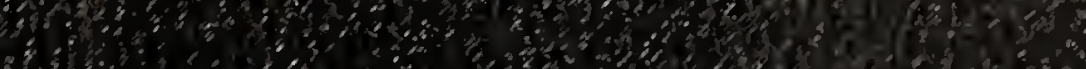
\%

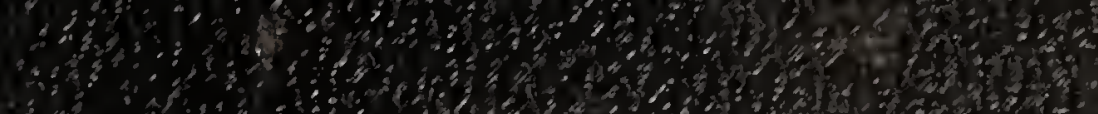

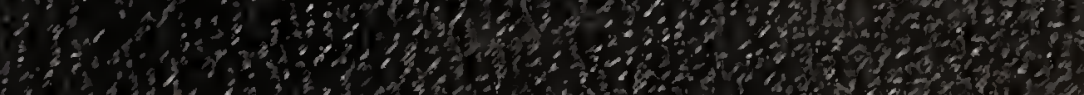
(3)

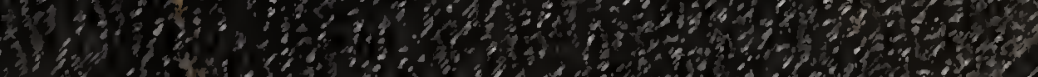

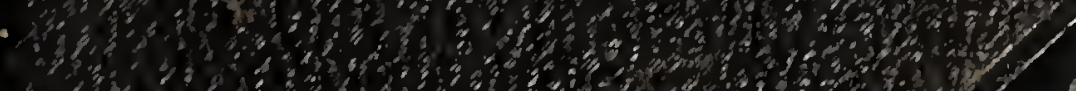
by

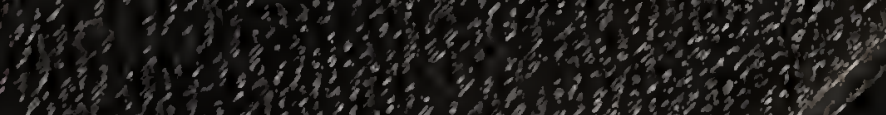



s
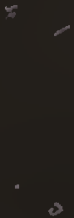

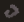

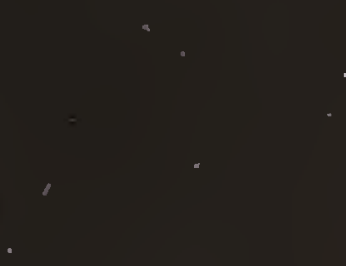

c. 

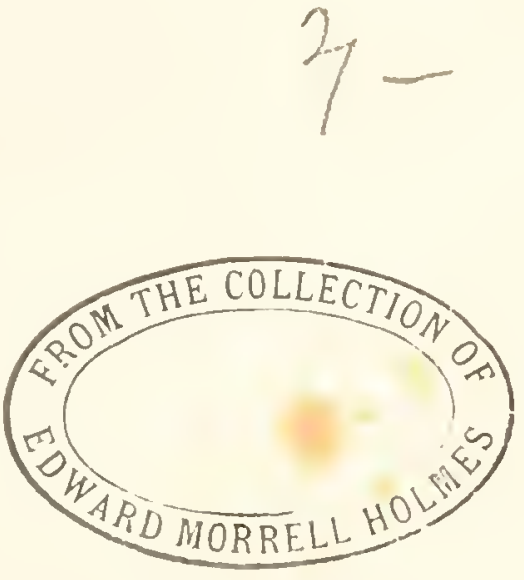


\section{SYNOPTICAL COMPEND}

\section{or \\ B R I T IS H B O T A N Y,}

ARPANGED AFTER TIH:

\section{LINNEAN SYSTEM ;}

ANU CONTAINING

THE ESSENTLAL CIIRACTERS̃ OF THE GENERA,

TIF SPECIFIC CIIARACTERS, ENGIISH XAHES, ILICES OF GROWTII, SOIL AND SITUATIUN, COLOUR OF TIIE FLOWERS, 'TMES OF FLOWEIING, DURATION, AND

RIFEREYCES TO FIGLRES.

ORIGINALLY

BY JOHN GALPINE, A. L. S.

\section{The $\sqrt{\text { Fourth }}$ Evition,}

INCLUDING

THE LINNEAX OUDER OF FEIXS, TOGETHER WITH ESSENTHAL CHAKACTERS AND TYPES OF TIE GENERA OF THE OTHEIR ORDERS OF CHY PTOGAMIA;

AND THE

XATURAL ORDERS OF THE GENEIRA IN ALL THE CLASSES.

\section{LONDON :}

SA $\|$ UE L, B A G S E R,

Paternosten Row, and, Vehe Street, Cavendish Square. 


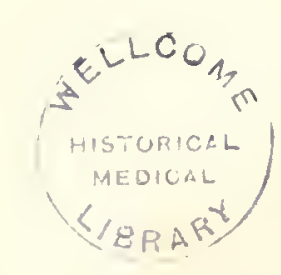


TO THE

PRESIDENT AND FELLOWS

oP

\title{
THE LINNEAN SOCIETY
}

OF LONDON,

\author{
THIS REVISED EDITION
}

OF A

\section{COMPEND OF BRITISH BOTANY,}

IS RESPECTFUILY DEDICATED,

BY TIEIR OBEDIENT SERVANT,

TIIE PUIBLISHER.

Yo. 15, PATERNOSTER ROW. 



\section{PREFACE}

\section{TO THE THIRD EDITION.}

Is the present state of botanical science, it has been thouglit advisable to add to the names of the Genera those of the Natural Orders to which they belong. In some few cases whcre botanists differ with regard to the genera in which a plant should be placed (as in the English Hyacinth, which has been repeatedly changing its place from IIyacinthus to Scilla, and from Scilla to IIyacinthus) the Editor has scrupulously followed the English Flova of Sir J.E. Smith: considering it important that students, for whom the work is especially adapted, should not be confused, alarmed, and detcrred by unnecessary contradictions, from even attempting to study the science. For the same reason Sir J. E. Smith's arrangement of the Filices has been adopted. The Musci, Hepaticere, Alga, and Lichenes, will be found to accord with Loudon's Encyclopedia of Plants, in which they are arranged by an able botanist, according to the latest and best authorities.

In consequence of the great attention latterly bestowed upon the Fungi, the genera are so considerably multiplied, as to becomc inadmissible without materially increasing the size of the volume. It is by no means certain whether sevcral of these are vegetables at all; and those which admit of more accurate classification, could be studied to no advantage from the specific characters of a few individuals: their inscrtion, thcrefore, in the manner followed with regard to the four preceding Orders, would afford no adequatc compensation for the increased bulk which would rendcr the volume less conveniently portable. This Order has not been wholly omitted; but one genus only has becn dcscribed of each subdivision in which the genera are ranged, instead of the types of the gcnera.

$$
\text { July } 7 t h, 1829 .
$$


Digitized by the Internet Archive in 2017 with funding from
Wellcome Library

https://archive.org/details/b29288770 


\section{MARKS AND ABBREVIATIONS.}

\section{MARKS.}

+ l'ut after the specific names of Plants which are not now found wild. s After the names of Plants which are doubtful natives.

! Ifter the Soil or Situation of suel Plants as are rare.

* In tle column for figures, denotes that the figure there quoted is given under a name different from that under which the Plant is deseribed in this work.

+ In the column for the soil, Sc, signifies or.

\section{SOIL, OR SITUATION.}

Alp. bogs ... Alpine bogs. Alp. bu.pl.. Alpine busliy plaees. Alp. hea. .. Alpíne heatlis. Alp.lak..... Alpine lakes. Alp. mar. .. Alpine marshes. Alp. mea. .. Alpine meadows. Alp.riv..... Alpine rivulets. $\Lambda$ lp. roc..... $\Lambda$ lpine rocks.

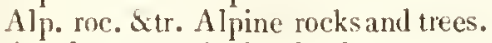
Ara. lan..... A rable land. Ba........ Janks. Bar. gro. .. Barren ground. Bar. hea. .. Barren heatlrs. Bar. past. .. Barren pastures. Bar.san. .. Barren sandy places. Boggy hea... Boggy heaths. Buggy in. .. Boggy mcadows. lioggy pl. .. Boggy plaees. Bo. on M. ? Bogs on Mountains. Bgs.on M. 3 Bogs 13or of fi. .. Borders of fields. Br. ...... Branehes. Bushy fi. .. Bushy fields.
Bushy hi. . Bushy hills. Bushy pl. .. Busliy places. Cale. ba. .. Calcareous banks. Calc. roc. .. Calcareous rocks. Chal. ba. .. Chalky banks. Clial.cl..... Chalky cliffs. Chal. fi..... Chalky fields. Clial. hil. .. Chalky hills. Chal. pa. .. Chalky pistures. Chal.so.... Chalky soil. Chal. wo. .. Chalky woots. Clov. fi..... Clover fields. Corn fi. .... Corn fields. Cult. gr..... Cultivated ground. Dit. ...... 1)itclres. Dit. ban. . Ditcl banks. Dry com. .. Dry commons. Dry fi. ... Dry fields. Dry liea..... Dry heatlıs. Dry m. pl. ? Dry mountainous I places. Dry pas. .. Dry pastures. Dry st. pl. .. Dry stony places. Dr. st. wds. . Dry stony woods. 
Dry wds. .. Dry woods.

Dunghit .... Dnunghills.

- Edges of D. . Edges of Ditches.

Grav. ban... Gravclly banks.

Grav. hea... Gravelly hcaths.

Grav. pas... Gravelly pastures.

Grav. so .... Gravelly soil.

Gro. Hed. .. Groves and hedges.

Hca. ...... Heatlis.

Ilea. \& wo. . Ilcaths and woods.

Hcd. ban. .. Hedge banks.

Highl. va. .. Highland valleys.

Hilly pas. .. Hilly pastures.

Ir. bogs .... Irish bogs.

Ir. mount. .. Irish mountains.

Ir. roc. .... Irish rocks.

Ir. shores .. Irish shores.

Ir. thick .... Irish thickets.

Lak. ...... Lakes.

Marg. lak. . Margins of lakes.

Marit. roc... Maritime rocks.

Mar. ..... Marshes.

Mea. ..... Meadows.

Mea. pas. . Meadows and pasturcs

Mic. rocks .. Micaceous rocks.

M. alp. pI... Moist alpine places.

M.alp. wo... Moist alpine woods.

Moi. chal. .. Moist chalky soil.

Moi. cha. h.. Moist chalky hills.

Moi. fi. .... Moist ficlds.

Moi. gr..... Moist ground.

Moi. lica. .. Moist hcaths.

Moi. hed. .. Moist hedges.

Moi. mea. .. Moist meadows.

Moi. pas. . Moist pastures.

Mioi. pl..... Moist plares.

Moi. roc. .. Moist rocks.

Moi. roc.S tr. Moist rocks and trees.

Mci.s. P. .. Moist sluady places.

Moi. wo. . . Muist woods.

Mos. bog. .. Mossy bogs.

Monut. .... Mountains.

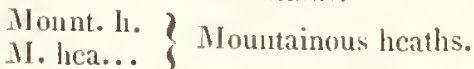

N. pas. .... Mountainous pastures.

Mou. pl. .. Monntainous places.

M.roc.hea $\left\{\begin{array}{c}\text { Mountainous rocky } \\ \text { leatls.s. }\end{array}\right.$
M. thick. . Mountainous thickcts.

M. wo. .... Mountainous woods.

Mud. dit. . Muklly ditches.

Mud.sh. .. Muddy slores.

N. of Eng... North of England.

N. of Sco... North of Scotland.

Nor. pas. .. Nortlern pastures.

Nor.wa.pl. $\left\{\begin{array}{l}\text { Watery places in the } \\ \text { North. }\end{array}\right.$

Nor. wds. .. Woods in the North.

Old w. ...

Old wa. .. Old walls.

Osicr hol. .. Osier holts.

Pas. ...... Pastures.

Peaty dit. .. Peaty ditches.

Riv. ban. .. River banks.

Rivul. .... Rivulets.

Ro. si. .... Road sides.

Rub...... Rubbish.

Salt mar. .. Salt marshcs.

S. W. dit. . Salt water ditches.

San. fi. .... Sandy fields.

San. gr. .... Sandy ground.

San. hea. . Sandy heaths.

san. mar. .. Sandy marshes.

San. pas. .. Sandy pastures.

San. pl. .... Sandy places.

San. sh..... Sandy shores.

San.w. pl. .. Sandy wet places.

San. t chal.. Sandy or chalky soil.

Sc. alps..... Scottish alps.

Sc. bogs.... Scottish bogs.

Sc. hea .... Scottish heaths.

Sic. isl. .... Scottisl islands.

Sc. mar.... Scottisl marshes.

Sc. mount... Scottish mountains.

Sc. pas. .... Scottish pastures.

Sc. roc. .... Scottish rocks.

Sc. sh. .... Scottivh shores.

sc.thick.... Scottish thickets.

Sc. val. .... Scotelı valleys.

sic. wo... I Scottish woods.

Sc.wds... ,

Sca co. .... Sea coast.

Siril. sh. ... Sea shore.

Siev. isles .. Severu islcs.

Sla. ba..... Sluatly banks.

shal bogs .. Shady longs. 
Sha. cops. . Shady copscs. Sha. la..... Shady lanes. Slı. m.pl... Shady moist places. Slin. pl. .... shady places. slia. roc. .. Shady rocks. So. coast .. South coast. S. of Sco. . South of Scotland. Sou. riv..... Southern rivulcts. Sp. bogs.... Spongy bogs. Stag. wa. .. Stagnant water. Ston. in wa. . Stones in water. Sto. \& wa... Stones and walls. Stony hi. .. Stony hills.

St. nnoor.... Stony moors. Stony pa. .. Stony paslures. Stony pl..... Stony placcs. Sun. hil..... Sunny hills. Sun, roc. .. Sunny rocks. Thick. .... Thickets. Tr........ Trees.

Tr. \& st.... Trees and stones. Tru. of trees, Trunks of trecs.
Tu. bogs .. Turfy bogs.

T'u. hea.... T'urfy heaths. Uncul.gr... Uncultivated ground. Uncul. pl... Uncultivatcd places. Wa. gr..... Waste ground. Wal. Bks... Walls and Banks. Wall. MIts... Walls and Mountains. Wat. com... Watcry commons. Wat. pl..... Watcry places. Wat. sh.pl.. Watery shady places. W. $\Lambda$ lps .. Welsh alps. Wel. bogs .. Welsh bogs. Wcl. lak. .. Welsh lakes. Wel. roc. .. Welsh rocks. Wct alp. hea. W'et alpine heaths. Wet. com... Wet commons. IV ct. gr..... W' Wret ground. Wet. gro. .. Wet groves. Wet. sa.p... Wet sandy places. Wet. sh. gr. . W' et shady ground. Wet. sha. pl. Wet shady placcs. Wet. thick.. Wet thickets.

\section{COLOUR OF TIE FLOWERS.}

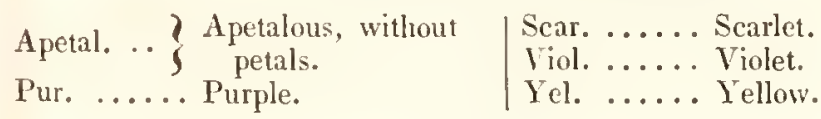

\section{TIME OF FLOWERING.}
l. January.
2. February.
3. March, Sic.

DURATION.
A. Annnal.
P. Perennial.
S. Shirub.
B. Biennial.
T. 'lree.

\section{DESCRIPTIONS.}

Androg. .... Androgynous.

Anth ...... Anther.

Bar. ...... Barren.

Bract. ... Bracta.

Cal. ..... Calyx.

Calypt. ... Calyptra.

Caps...... Capsule.
Cor. ...... Corolla.

Corymb..... Corymbus.

Cylind. .... Cylindrical.

Dichot. .... Dichotomous.

Fem. ...... Fenalc.

Fer. ..... Fertilc.

Filam. .... Filament. 


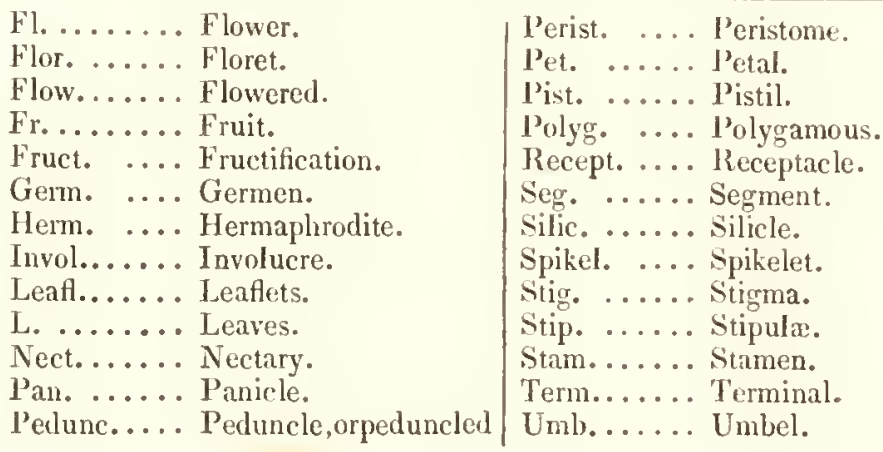

\section{BOOKS QUOTED.}

Achar . . . ....... $\}$ Acharius, Lichenographia Universalis. Amals of Bot. ...... Annals of Botany by C. Konig and J. Sims. Bot. Mag.......... Curtis and Sim's Botanical Magazinc.

De Candolle ..... De Candolle, Flore Française.

Eng. Bot. ......... Smith and Sowerby's Euglish Botany.

Eng. Flo. ......... Smith's English Flora.

Flor. Dun........... Icones Plantarum sponte nascentiun in Reunis Dania et Norwegia:

Gray's Brit. Pl....... Gray's Natural Arrangement of British Plauts. Mowiter's Musc. Brit... Ilooker and Taylor's Muscologia Britannica. Mrrt. Kew.......... Ilortus kewensis. Hel. 2.

Mudson ........... Hudson's Flora Anglica.

Iussien .......... Jussieu, Genera Plantarum.

Lam.......... Lamouroux in Annales du Museum d'llistoire Lamouroux....... j naturelle.

Limd. Sym.......... Lindley's Synopsis of the British Flora.

Linn............ Linnai Genera Plantarum.

Iondon's Ency. P'l. . Loudon's Fncyclopedia of Plants.

Plık. Phyt. ....... Plukenet, Pliytographia.

Suc. Eng. Fung. . . . Sowerlyy's Coloured figures of the English Fungi or Mushrooms.

Trams. Lim. Soc. .... Transactions of the Linnenu Society of Lonlon.

Ventenat.......... Ventenat, Choix des plantes.

W'illd. ......... Wildenow, Species plantarum. 


\section{BOTANICAL TERMS.}

ACEROSE, chaffy

Acicular, needle-sloaped

Aciniform, scimitar-sliaped

Aculeate, prickly

Aduate, athering

Aggrtgate, many fowers included in one common calyx

Alate, winged

Androgynous, bearing wale and female Howers, distinct from each other on the same root

Anther, the summit of the stameu, con taining the pollen

Apetalous, without petals

Apophysis, a swelling beneatl the capsule of a moss

Appressed, squeezed close

A rborescent, becoming woody

Arboreus, woody and permanent

Arcuate, bowed, bent like is bow

Articulated, jointed

Asci. small tubes in which the seeds of some Cryptogamic plants are placed

Atteuuated, growing slender

Auriculated, ear-shaped

Awn, or beard, a sharp process, issuing from the chaff in corn and grasses

A wnless, withour beard

Axillary, thowers, 8 c. proceeding from als angle formed by a branch with the stem

Beaded, granulated

Beaked, projectiug like the beak of a bird

Bearded, heset with parallel hairs, or tufts of hair

Bicapsular, having two capsules

Bieuuial, plants which continue alive two years

Bifid, two-cleft or cloven

Bifarious, pointing from opposite sides

Bilabiate, blossom with two lips

Bilocular, having two cells

Binate, in pairs

Bipartite, deeply divided into two parts

Bipinate, doubly-winged

Biternute, doubly three-fold

livalve, two valved

Border, the upper spreading part of a monopetalous thower

Bractea, foral leaf, may often be mistaken for the calyx, but is more permanent

Bulbiferous, Howers succeeded by bulbs iustead of seeds

Calyptra, veil, coveriug the anther of mosses like a hood

Calyx, the outer covering of the flower

Campanulate, bell-sliaped

Canaliculate, channelled

Cancellaterl, latticed

Capillary, liair-like

Capitate, growing in heads

Capsule, a membranous hollow seed re sel.

Carinated, kecled

Carnose, fleshy
Catkin, tlowers and chaff arranged on a long slender stalk like at cat's tail

Caudite, tailed

Caulescent, having a stem

Cell, the lollow in a capsule for lodging the seed

Centr,1] florets, those which occupy the misldle part of a compound flower

Chatt, the ary calyx of corn and grasses

Ciliate, fringed

Cirrlii, tendrils

Cirrhose, terminating in a tendril

Clavated, club-shaped

Claw, the lower narrow part of the petal in flowers

Clelt, divided into parts

Club-sbaped, thinner at the base than the termunation

Coadunate, joined together at the base

Coated, composed of layers, as the bulb of the onion

Cochleated, snail-shaped

Column, the ceutral pillar in a capsule

Columuar, like the sliat of a column

Common fruit stalk, bearing several flowers

Compact, growing close, aud as it were pressed together

Complicate-carinate, doubly keeled

Compound Hower's, consisting of many florets or little flowers

Compressed, a stem baving the two opposite sides, plain or that

Conduplicate, folded, or doubled together

Contluent, running one into another at the base

Congested, heaped togetluer

Conjugated leat, a pinuate leaf with only one pair of leaflets

Connate, united at the base

Connivent, converging or approaching

Converging, applied to the corolla, when the tips of the petals meet so as to close the Hower

Convoluted, twisted spirally

Cordate, heart-shaped

Cordate-lanceolate, heart spear-shaped

Coriaceous, leather-like

Cornute, horn-shaped

Corolla, blossom

Corona, crow!

Corymb, a bunch of flowers supported on separate foot-stalks

Costated, ribbed

Cotyledones, seed-lobes

Crenate, seolloped

Crenato-serrulated, finely notched

Crested, laving an appendage like a crest or tuft

Criuite, hairy

Cruciform, cross shaped

Cucullate, cone-shaped

Culın, striw

Cuneitorm, wedge-shaped

Curtail, the caly $x$ of agarics 
Cilspirlate, prickly pointed

Cuathitorn, rlass-shaped

Cylindrical, round witlout angles, like a walking stick

Cyme, tuft

Cymose, tulted

Deciduous, falling of

Declining, bent like a bow with the arch downwards

Decompound, doubly compound, or con. taining within a common calyx, smaller calyxes common to several thowers

Decumbent, tying down

Decurrent leaf, having its base runniug rlown the stem

Decussated, crossed at right angles

Deflected, bowed or bent down

Dehiscent, zaping

Deltoid, trowt-shaped

Dentate, toothed

l)enticuliate, set with little teeth

Dichotomous, torked

Didymous, double

Digitate, finger-like

1) Inistate, divided into two parts

Dioecious, male and female tlowers, growiug on difterent plants

Dipetalous, of two petals

Diphyllous, two leaved

Disk, the whole surtace of a leaf or flower

Divaricating, stradelling

Diverging, making a right angle

I) r upe, a pulpy seed vessel, containing a nut or stone

Duplicate, doubled

Echinated, set with prickles

Elliptic, ovit

Elliptic-lanceolate, narrow oval

Emarginate, notched at the eud

Ensitorm, sword-sliaped

Erose, gnawed

Exserted, protruded, opposed to inclosed

Extra-foliate, growing under the leaves

Firina, sce pollen

Fiscicled, bundled

lastiviate, Hat topped

leathered, downy

liemale tlowers, containing pistils but no stamens

Fibres, woody strings or nerves

f'ilament, the thread-like part of a stamen, silpporting the anther

I iliform, thread-shaped

istulous, hollow

filexuose, riz-zas, winding

Foral leit, the leat next the nower, sometimes mistatien for the calyx

loret, the separate little flower of all aggregite flower

Poliaceous, leaty

liroul, when the stem, root, and leaf, are all in one, as in ferms

Iruit-sta!k, astalk letaring flowers but not leaves

Fiuticose, shrub-like

Juliginous, sooty

Furcated, forty

lisitorm, spindle-straped

(ieniculated, knee-joluted

(itemen, or seed bud, the rudiment of the Iruit yet wn embiryo

Gibhous, bulain:

ribls, the thu plites on thr under side of it inushroom
Glambs, secretory vesuels

(ilandular, formed of glands

Glaucous, grey blueish green

Glomerate, flowers growing close together in a globular form

C: lume, husk

Grinslated, beaded

I astate, halbert-shaped

Ilastate-sinuaterl, jagged halbert-shaped

Herbaceous, succulent and tender, in opposition to woody

Ilermaphrodite flowers, containing botls stamens and pistils

llirsute, rough with strong hairs

llispid, bristly

Husk, dry semi-transparent membranes, lorming the calyx and blossoms of grasses

IIynenium, that part of a fungus which contains the reproductive organs

I mbricated, tiled

limpertiect Howers, such as want either anther or pistil, or both

Incise, serrated, gashed

Incrassated, thickened upwards

Incumbent, leaning or restung against

Incursed, bowing inwards

Indented, liollowed or deeply scolloped

listlected, bent inwards

Infundibutitorm, tunnel-shaped

Internode, the space between the joints

Intrafoliaceous, within the leaves

Involucrum, the calyx of an umbel

Involucret, a pas tial involucrum

Involute, rolled inwards

interrupted, divided by intervals of smilter flowers

Keeled, bent like the keel of a ship or boat

Tahiated, having lips

Laciniated, jagged

lactescent, milky

lamellax, the rills of a fungus

Laminated, the that surface of leaves lying close upon each other

Tanceolate, spear-shaped

Ianceolate-ovate, spear-egg-shaped

Leatlet, or little leat, one of the single leaves of a compound leaf

legumen, pod

Lenticular, spherically convex on both sides

Lid, a cover to the capsules of mosses

Lignowe, woody

I.mb, the upper flat broad part of the pe. tal is called the limb

Iine:ur, strap-shaped

linear-lanceolitte, strap spear-sliaped

lineated, streatied

Lip, the upper or under division of a gaping blossom

Lobed, divided nearly half way down into lobes

I.unated, crescent.shaped

lyrate, lyre-shaped

Male tlowers-Howers containing slatnens but no pistuls

Marbinate, bolitered

Mid-rıb, the principal nerve iu a leat

Mitritorm-nutre-formed

Moniliform, necklace-shaped, like be'stls strumer tosethes

Monopetialous, (tlower) one petaled

Vomonlyyllous, one teated 
Honuspermous, une seederl

Mucronated, sharp-pointed at the end

Multangulat, uawy-cormered

Iuricated, covered witl sharp points

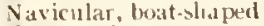

Vectary, honey cup, a part of a llowel deslyned to secrete and continill lioney

Neutral tlowers, without stamen or pistils. and producing 110 seeds

Node, knot

Nucule, a little put

Ubconic, inversely conic

Ubcordate, inversely heart-shaped

Obovate, mversely egs-shaped, that is, with the small end down wards

Obsolete, malistiuct

Octotid, ergbt-cleft

Olivaceous, liaving the qualities of olives

Operculum, lin

Orbiculate, round and tat

Osseus, hard as bone

Ovate, egg-shaped

Orate-linceolate, egrs-spear-shaped

Palate, the inner pait of the mouth of gapiug blossoms

Paleaceous, chatly

Palmate, hand-shaped

Panduritorin, fiddle-shaped

Panicle, an assemblage of flowers growing upon subdivided truit stalks, as in oats and some grasses

Papiliouaceous, butterfly-shaped

Papillose, pimpled

Parasitical, growing on some other plant, not on the ground

Partite, divided

Pectinated, comb-like

Pedate, bird-iooted

Pedicle, a partial peduncle, or little fruit stalk

Peduuculate, growing on a fruit stalk

Peduncle, fruit stalk

Peltate, whel the stalk is fixed in the disk, instead of the maruin

Pendulous, langing down

Pentagonal, five-cornered

Pentapetalous, tive-petaled

Pentaphyllous, five-leaved

Perennial, continuing for several years

Perfect Hower, haring both stamens and pistils

Pertorated leaf, full of small holes, very apparent when held up to the light

Perfoliate, the stem passing through the leat'

Perianth, cup or calyx

Pericalp, seed vessel

Perichatial.leaves, leaves surrounding the base of the truit stalk in mosses

Peristome, the rim surroundiug the inoutl, of the capsule in mosses

Permanent, applied to calyxes, \&c. remainiug on the plant till the fruit is ripe

Personate blossom, gaping

Petaliform, resembling a petal

Petals, the coloured leaves which consti. tute the blossoun

Petiolate, having leaf-stalks

Petiole, a leaf-stalk

Pileus, the cap of a fungus

Pilose, haily

Pinuatifid, teather.cleft

Pinnated, winged, a compound leaf formed ot several little leaves fastened to eacli sicle of it

Pinmulate, when each pinns is sublivided

l'istil. a part of the flower standing in the centre and composed of the gern, style, :lld summit

Plicate, plaited

Plumose, teathered

Pod, a seed re'sscl

Polleu, the dust contained in the anthes of fowers

Polypetislous, mauy-petaled

Procumbent, trailing

Prolilerous, wheu one blossom grows out of another

Pulvescent, covered with solt wool or hair

Punctated, dotted

Quinate, growing in fives

Raceme, a cluster of flowers, which is attached by a pedicle to a connous stalk; as in currauts

Rachis, the common stalk, or recept. to which the Howers of grasscs, \&c, are attached

Radiate, compound flowers in which the florets in the centre differ from those of the circumference

Radical, issuing immediately from the root

Radii, the outer florets of a radiate compound Hower

Ramose, branched

Receptacle, the base upon which the other parts of a Hower are placed

Reclining, bent downwards so that the point of the leat is lower than the base

Recurved, curved downwards

Reflected, bent back

Reniform, kidney-shaped

Repens, creeping

Replicited, folded or plaited

Reticulated, veined like net-work

Revolute, rolled back

Retroflexed, distorted

Rhomboicl-triangular, three colnered dia moncl-shape

Rhomboid-ovate, egg-dianoud sluaped

Ringent, gapiug

Root-leaves, leitves proceeding inmediateIy from the root without the interven. tion of a stem

Rostrate, beaked

Rotate, wheel-sliaped

Rugose, wrinkled

Runcinate, notched, the notches pointing backwards

Sagittate, arrow-sluaped

Salver shaped, a blossom tubular at bottom, Hat and expanding at top

Scaber, rough like a tile

Scape, stalk

Scious, sloots

Scariose, skiuny

Senibifid, lialf-cleft

Semicylindrical, balt a cylunder divided lengthwise

Seminal leaves, arising immediately from the seed-lobes

Serrated, toothed like a saw

Serrulated, very minutely serrated

Sessile, sitting

Setaceous, bristle-shaped

Setose, bristly

Sexangular, six-sided

Sliaggy, rough with stiff hairs 
Slueitly, a nembrane mvesting a stem or branch

Shicile a circular kind of fructification of some lichcas

Shrubly, pertmial with sevcral woody stems

Silicle, a little pod

Silıque, por

Simple, undivided

Sitting, leaves haviug no leaf-stalk, or flowers without it irujt-stalk

Spadix, sheatlied iruit-stalk

Spathe, sheath

Syatulate, battleilore-shaped

Spike, sessile flowers placed altermately on each side ot the truit-stalk

Epikelet, a little spike

Spindle-shajed, gradually tapering

Spinose, thomy

Sporidia, small transparent bodies contain. ing seeds

Spur, shaped like the spur of a cock

Squanose, scaly

Stantens, long slender bodies placed in the Hower cup round the pistul

Standard, the upricht petal of a butterfly. shaped blossoni

Stellate, starry, leaves surrounding the stem in a whirl

Stem.leaves, growing immediately upon the stem

Stigma, the summit of the pistil

Stipes, the stalk of a fungus

Stipitate, standing on a pedicle

Stipula, small leaves or scales at the base of the leat stalk

Stoloniferous, putting forth suckers

Strap-shaped, long and narrow

Striated, scored witl slender lines

Style, the inidrlle portion of the pistil, con wecting the stigina with the germ

Subcymose, some what tutted

Subrimose, a little branched

Subserrated, somewhat seirated

Subilate, awl-shaped

Succulent, juicy

Sulsessile, almost sessile

Sulliticose, somewliat wondy

Summit, the top of the pistil

suture, seam

Sword-shaped, long, narrow, and pointed
I emdril, at sparal shoot

I ermiltal, elution a brancli or stem

"lennate, leaves, yrowing three togetier trmm the same point

l'esselate, chequered

I'lallus, the part which bears the tructification, in lichens

Theca, the seed-ressel of Cryptogamic plants

lilell, one leat or scale partly covering another

Iomentose, cotiony

linifi, three-clett

Jivalved, three valved

- Truncate, lopped

Junirate, coated

lurbiluate, top-shaped

Uimbel. is composition of flowers, in which a number of slender fruit.stalks pro. ceerl trom the sane centre, like the spokes ot an umbrella

Uncunate, hooked at the end

Lindate, waved

Undilated, wated

Unequal thorets, when those in the circum. frence of un tmbel, ale larger than those in the centre

Ungulate, hool's! itped

Uuwalve, one-valved

Urceolate, pitcher-sliapel

Vaginate, sheathed

Valves, the several pieces which compose a capsule, alon the leaflets conposing the calyx aud corolla, in grasses

Veil, the calyx of mosses

Ventricose, distemuled

Verrucost, warty

Verticillate, growing in whirls

Vesicles, bladders

Villous, sott-llaired

Volva, wrappel

Whirls, leaves or flowers, surrounding the stem in a ring

Wiugs, the latiol petals of a butterflyshaped blosson!

Winged leat, formed of nany little leaves growing liom each side the stalk

Wing-clett, a leaf cut deeply down the midclle

If colly, clothci with a puhescence rescm. bling wool

\section{CLASSES AND ORI)YRS.}

\section{CLASSLS}

Monandria, (from wouns onc, and ayse a male) the titst class, compreliending plants with one stamen only

Diandria, (fiom sos two, und avep a male) plants with two stamens

Triandria, (from tous three, and alep it male) plints with tluce stamens

Tetraudria (tronl efoodes tour, and avep at male) plants witl four statueus

Pentandria, (from лерт live, and avep a male) plituts with hive stamens

llexandria, (from of six, and ove i male) plants with six statmens

Ileptandria, (from into seven, asd abe a male) plants with scren stamens

Octandria, from orsw ejglit, and ave al male) plants witli eiglat stamens

Enneandria, (from erva uine, and owp a male) plants with nine stamens

Decandria, from dna ten, and ayp a male) plants with ten stamens

Dodecandria, (fiom soudno twelve, and avep d male) plants with twclve stimens

Icosimblia, (from nososi twenty, and asep at Inale) plants with twenty stamens

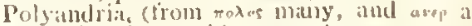
male) plants with many stamens

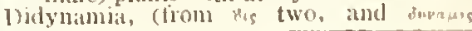


nower) plauts with four stamelts, two longer and two shorter

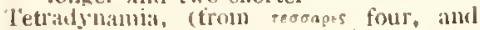
sururs powere plants with six stamens, fon longer and two shorter

Monallelphiat, from rovos one, and ase $\lambda$ pos * brotherhond) plants with the staneus all united

Diadelphiti, from ans two, and asedpos a brotherioml) plants with the staneus in two bodies

Polyadelphia, ftrom roגws many, and ase $\lambda \phi o s$ a brotherlood) plants witl the stameus in three or more parcels

Syngenesia, (tronl owy together, and yovors generating) plants with the anthers united into a cylinder
Gymandria, (lious rovy at woman, and avep it male) platuts in which the statnens grow upon the pistil

Monercia, (from powns alone, and orkots it house) plants producing male and fenuile thowers separate, on the same indivitual

Diccia, (from sis two, and oros it louse) plants producing male and fenale Howers on distinct individuals

Polygamia, (from roגes many, and yauns a marriage) plants bearing hermaphrodite Howers, with male or female How. ers, or both

Cryptogamia, (from $к р и \pi т \omega$ to bide, and you 5 a marriage) plants in which the fructitication is concealed

\section{ORDERS.}

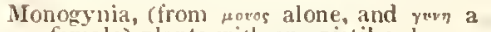
feniale) plants with one pistil only

Digynia, (from sis two, and $y^{\prime \prime n}$ a temale) plants with two pistils

Trigynia, (from spsis tliree, and yor a female) plants having three pistils

Tetragynia, (trom reorapes four, and yom a female) plants with four pistils

Pentagrnia, (from revre five, and rey a female) plants with five pistils

IIeptagynid, (trom $\varepsilon \pi \div a$ sevel, and yon a female) plants with seveu pistils

Decagynia, (from $x_{x \rightarrow}$ ten, and yoy a female) plants with ten pistils

Dodecagynia, (from soossa twelve, and yerm a temale) plants with twelve pistils

Polyeynia, (from $\pi \lambda^{2}$ us many, and ruv a female) plants with many pistils

Gynnospermia, (from row wos naked, and orepua seed) plants with four naked seeds

Angiospermia, (from aryos a vessel, and $\sigma \pi \rho \rho \mu$ seed) plants of the lith class, in which the seeds are enclosed in a capsule

Siliculosa, (the dim. of silinua a pod) plants of the 15 th class, in which the seed vessels are generally roundish

Siliquosi. (from silzgna a pod) plants of the 15 th class, in which the seed ressel is very loug

Polygamia Fqualis, plants of the 19th cldss, in which all the florets are per. fect, aud the whole flower regular

Polygamia Superflua, plants of the 19th class, witl perfect florets in the disk, and pistilliterous florets in the ray, both bearing seed

Polygamia Frustranea. plants of the 19th class, having perfect Horets in the disk, producing seed, while those iu the ray are imperiect, and without seed

Polygamia Moncecia, plants of the 23rd class, with perfect and innperfect flow. ers on the same individual

Filices, (Ferns) plants of the 24 th class, bearing steds on the back of the leaves

Musci, (Mosses) plants of the 94th class, with imperfect flowers, with the seeds in a capsule, often covered with a veil

Hepatica, plants of the 24 th class, partaking of the characters of the Musci and Alure

Alge, plants of the sth class, always aquatic

Lichene's, plants of the 24th class, with the fructification usually dispersed over the surface of the trond, or thallus. 'lley are found chietly on walls, stones, the bark of trees, or decayed wood

Fungi, ptants of the attl class, with no discernible thowers, but seerls in the gills, pores, cups, \&c., holding the lowest ranh in tle vegetable kinglom.

- The last three genera placed under this Order are exceptions, and are by most botanists

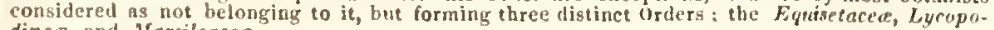
dinea, and Marsileacea. 



\section{PREFACE}

\section{TO THE FOURTH EDITION.}

Ls again presenting this little volume before the public, we are aware that has disadrantages to encounter in the present fashion of undervaluing the stem by which it is arranged. Even Science is under the dominion of shion; but she, like the moon, is proverbial for her fickleness, and we do t despair of seeing the Linnean system again honoured by her patronage. is too radically strong to sink for ever under the rude weapons aimed sinst it, and we hope ere long to see it go hand in hand with that which vuld be its brother, not its rival system. Both have their merits, both $y$ be studied with advantage : their purposes are different, but not incomtible, as was show long since by Mr. Bicheno.

According to the disciples of Inssieu, it would appear that the Linnean :tem was strangely limited: it would seem that accurately to ascertain the me of a plant, was to deprive ourselves of the power of acquiring any -ther linowledge concerning it. This is a singular notion. As well might say that loy tilking the most direct road in travelling towards a foreign untry, we becune incapable of exploring it when we arrived; but were mpelled to stand at the entrance, boasting of our familiarity with its uabitants, and lnowledge of its topography. As well might we say that grammatical knowledge of a foreign language incapacitated us for readthe author's who had written in that language. The Linnem system is the :mmar of the science, the Jussieuan is its literature. The studeut should mmence with the former. Botany, however studied, is still the sime sci.e; it includes all that can be learned respecting the vegetable world. One m inay choose to travel in the dark, anotler may choose to furnish himself th a light to guide him; ind, if he pleases, the former may value himself on the difficulty he finds in groping his way, as upon a merit. 'The very tuty and excellence of the linuean system is made a sulject of reproach; 1 because its rudiments are clear, and the student early finds himself a :ster of the ineaus by which he works, he is ridiculed, as fancying himself possession of lnowledge which he has not. 'The undervaluers of Linas have a curious mode of comparing the systems. By the limmean, say $y$, a man may know an infinity of names, but no more; while by the sural method he cannot ascertain one plant without acquiring much wlerge respecting it. The fact is, that the one system proceels from mes to things, the other from things to names. To us it sepms that the tree of hnowledge necessarily preceding the ascertaining of il plant, is a iect. So thought Mr. Roscoe: "Is it no disidvantage," says he, "on covering an unknown plant to be under the necessity, before we proceed its further investigation, of uscertaining in what manner it commenced its "wth, and whether it rose from the seed with one or with two cotyledons, without any cotyledon whatever? To whom are we to apply for this inmation? or are we to turn round to ascertain the primary distinction by 
the sensible appearance, and instead of saying that the plant rose from one cotyledon, and therefore is a grass; that it is a grass, and therefore rose from
one cotyledon?"

A student of the Linnean system will for a time confine himself to ascertaining the names of plants; having acquired the power of doing this with facility, he passes onwards witl rapid strides. It is said that the fastest walkers commence the exercise at a molerate pace; so is it with the disciple of Linneus. The reproach against the system, that its followers know nothing but names, is an unjust one. It is not to the system, but to its superficial students, that the reproacl applies; and if fewer of Jussieu's disciples are found to possess a very limited knowledge, it is, perhaps, because such persons are deterred by the diticulties of his method, from even at tempting the study.

It has been asserted that Linneus contemplated the discovery of a natural method, and considered an artificial system as merely introductory to it. Very true,-but he did not mean that the latter was to be superseded by it. It was to continue to be the introductory means of studying the science-the rudimental part of it. What else does he mean by tlie following passage?-

"Natural orders cannot constitute a method without a liey. In distinguishing plants, the artificial method alone is of any avail; a natural method being scarcely, if at all, possible. Natural orders are useful in acquainting us with the nature of plants, but an artificial method is necessary to their discrimination."

It has been said that Linneus, while he demonstrated plants by his own artificial system in his public lectures, developed the principles by which lie was guided in the construction of his natural orders, in lis conferences with his more distinguished pupils. What can more clearly prove that what is treated now as the extent of Linnean botauy, was at all times considered only as its commencement?

Much has been effected in favour of the Jussieuan method, by the use of the word natural; but it would puzzle Solomon to discoser in what respect his method is more natural than that of his predecessor. Mr. Roscoe, in the admirable papers before mentioned, lias very clearly sliown that both are equally artificial, and that the Linnean is greatly superior in many respects. The nethod lias doubtless been considerably improved since those papers were written, but we see not that it is a jot the more natural. We would recommend that the Linnean system should be followed up ly the study of the Iussieuan, not because it is a natural method, but because it is a definite mode of studying the physiological part of the science, and because the attempt to arrange plants according to their natural atinities necessarily accustoms the student to consider those affinities, and tends to prepare the way for a natural metlod.

Would our limits permit, we would willingly reprint the whole of $\mathrm{Mr}$. Roscoe's papers on the merits of the two systems: we luust content ourselves with referring such of our readers as may not be acquainted witl them to the "Transactions of the Linnean Society" for 1810 , or to the "Philo sophical Mtagazine" for 1830, in which they were repriuted.

May 1st, 1834 . 


\section{CLASS 1 .}

\section{MONANDRIA.}

\section{ORDER I. MONOGYNIA.}

* Seeds naked.

I Siliconsi. Cal. ventricose, entire. Cor.0. Stam. 1 or 2. Seed 1, in the bottom of the calyx.

2 Hipuris. Cul. a slight border. Cor. 0. Seed 1, inferior.

Taleriana 1. Alchemilla 3.

* Secds capsular.

3 Cuna. Berry many-scederl. Stule 0.

4 \%ostera. Caps. 1 -secded. Spadir flat, many-flowered. Drupa. nut with one kernel. Stig. 2.

Salix occasionally.

\section{ORDER II. DIGYNIA.}

5 Callitriene. Cal.0. Petul 2, inferior. Seeds 4, naked, marginate on one side. Flowers, some monoceious. 
(2)

LINNEAN NAMES.

ExGLISI NAMES.
SAMPIIRE.

\section{SALICORNIA.}

1 lierbacea......

marslı

2 procumbens ...

procumbent.....

3 radicans......

4 fruticosa......

creeping-rooted ..

\begin{tabular}{|c|c|}
\hline 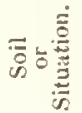 & $\frac{5}{3}$ \\
\hline
\end{tabular}

1.

Sea sh. apetal. $8,9 \quad 1$

Salt mar...... 8 A

2475

shrubby .

IIIPPURIS.

1 vulgaris

MARE'S-TAIL.

CIIARA. common

CIARA.

1 vulgaris con

$$
2
$$

2 hispida

3 Acxilis ......

- prickly .......

.

I

. smooth ........

4 translucens.... transparent.....

5 nidifica ..... prolifcrous ......

P

6 gracilis ......

ZOSTERA.

GRASS WRACK

1 marina common ........

Sea sh.

P. $\quad 1691$

- Sea sh.

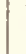

Ditches

Pools

I

\begin{tabular}{c|c}
$\ldots . .$. & 8 \\
$\cdots$ & 9
\end{tabular}

P. 2467

P.

763

apctal. 5,6

(1)

A? 336

1. 463

A. 1070

1. 1855

A. 1703

Pools!

9 A.

2140

Sca sli. apetal. $8,9 \quad \mathrm{P}$.

ORDER IT. DIGINIA.

6 CALLITRICHE.IWATER-STAR-WORT.

I verna ....... vernal ........ Ditches white 4,5 A. $72: 2$

2 autumnalis.... autumnal ..... lakes 
1. SALICORNIA. Chenopoded. R. Br.

1 Hcrbaccous, erect ; internodes obconic. joints compressed, notched : spikes tapering.

2 Hcrbaceous, procumbent; intcrnodcs obconic. branches simple: spikes tapering: 2 stamens.

3 Woody, procumbent; intcrnodes nearly cylind; joints compressed, notclied: spikes oblong: 2 stamens.

4 Woody, ascending; joints and internodes cylind: spikes nearly sessilc, cylind. obtuse.

2. IIIPPIRIS. Halorager. R. BR.

1 L. verticillate, lincar: Fl. scssile, one in the boson of each leaf.

\section{CIIARA. Naiades. Juss.}

1 Striated without prickles; leaves 0 . whorled branches tapering, with internal partitions: bracteas 4 . together.

2 Furrowed : leaves 0. whorled branches with internal partitions: bracteas verticillate : stem prickles bristly, deflexed.

3 Smooth, transparent, without prickles: leaves 0 . whorled branches cylind.obtuse, mucronulated, without internal partitions: bracteas 0 .

4 Smooth, tramsparent, without prickles: leaves 0 . whorled branches simple, cylind. obtuse, mucronulatcd, with transverse internal partitions : bracteas 0 .

5 Smooth, transparent, without prickles: leaves 0 . whorlcd branches simple, cylind. clongated, without internal partitions: anther sometimes stalked: bracteas unequal.

6 Smooth, transparent, shining, without prickles: branches dichotoinous, acute: lcaves 0 . bracteas 0 .

\section{ZOSTERA. Fluviules. Rici.}

1 Caps, sessilc: I. altcrnate, linear, long, flaccid : stem slightly compresscd.

\section{ORDER II. DIG INA.}

\section{CALIITRIClIE. Haloragea, R, Br.}

1 L. opposite: Fl. axillary, solitary, sessile.

2 L. triple-ribbcd; the uppermost crowded, obovate: margin of the seeds obtuse. 



\title{
CLASS 11 .
}

\author{
DIANDRIA.
}

ORDER I. MONOGYNIA.

*Fl. inferior, 1-pet. regular.

6 ligustrum. Cor. 4-cleft. Berry 4-seeded.

7 Finsixes. Cor.0. or 4-parted. Cups. eompressed, 2-sceded $F l$. some female.

* Fl. inferior, 1-pet. irregular, capsular.

9 Vinoxres. Cor. 4-parted, wheel-shaped. Caps. 2-celled.

10 l'nsicicula. Cor. ringent, spurred. Caps. 1-eelled. Cal.5-eleft.

11 Utucularia. Cor. ringent spurred. Caps. 1-celled. Cal. 2-leaved.

*** inferior, 1-pet. irregular: seeds naked.

13 Licorus. Cor. nearly equal, 4-cleft, one lobe notehed. Stam. distant, simple. Seeds retuse.

14 S.ILria. Cor. ringent. Filum. fixed trausversely on a little pedicle.

****s. superior.

8 Cincas. Cor. 2-pet. Cal. of 2. seg. Caps. 2-eelled. seeds solitary.

米米类草 Fl. apetalous, or 4 -petalled.

12 Illaxa. Cor. 0. Cal. l-leaved. Cups. 1-seeded.

15 Chabrim. Cor, 0, Glumes ehaffy and sheathing; the outer ones empty. Drupa, without bristles at the base.

Salicornia 2, 3. Fraximus 1. Schanns 4. Carex. Lepidium 2. Coronopus 2.

\section{ORDER 11. DIGINIA.}

16 Anthuxantiua. Cal. glume 2-valved, 1-flowered. Cor, glume 2 -valved, awned. Seed 1.

Hierochloe. n. 41 . 


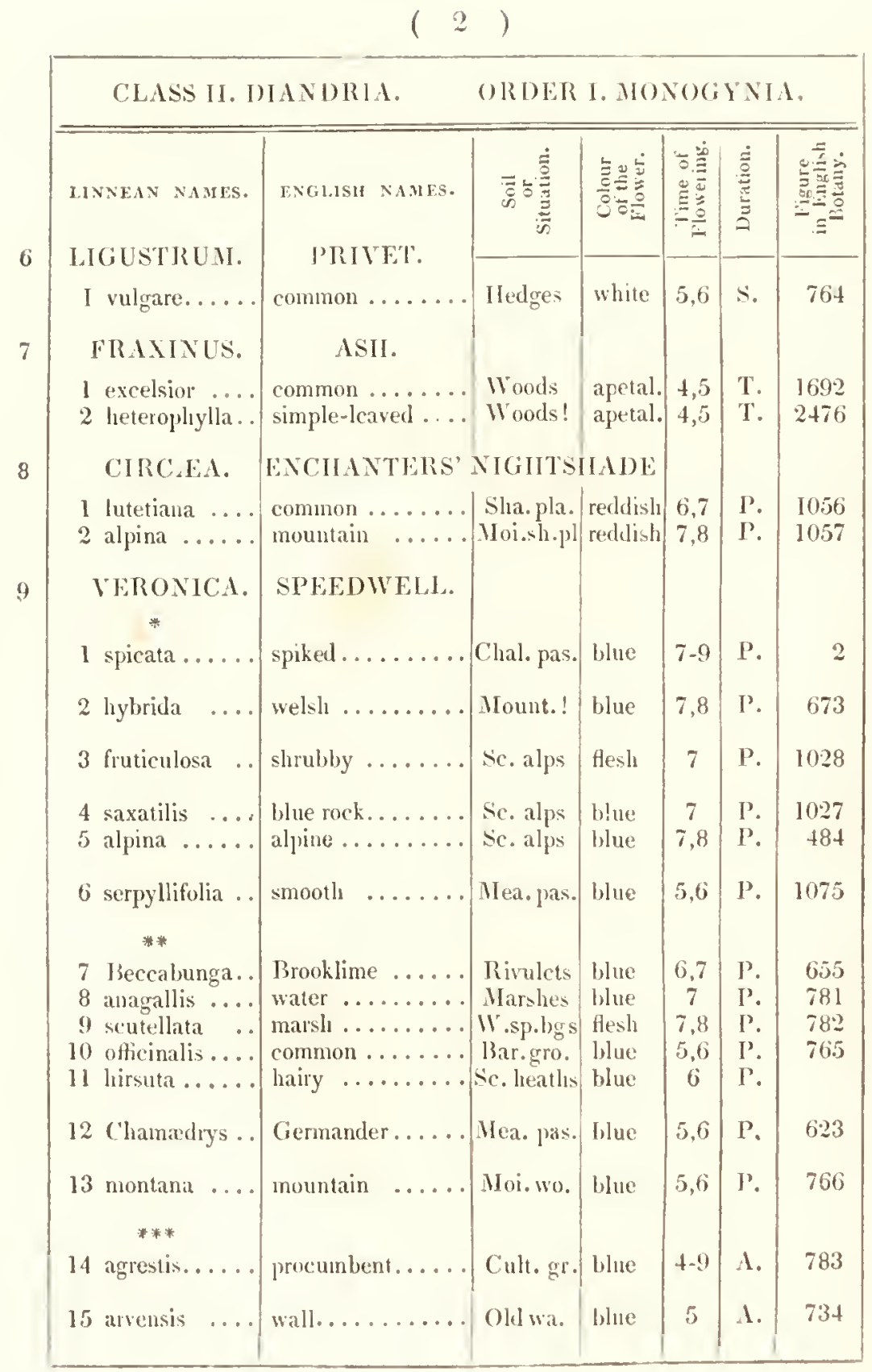


SIECIFIC CLARACTLR.

6. LIGUSTRUM. Oleinea. R. BR.

1 1. clliptic-lanceolate, obtuse, mucronulated.

7. FRAXINUS. Oleiner. R. Br.

1 l.eaf. serrated: Fl. destitute of calyx and corolla.

2 L. both simplc and compound, with tooth-like serratures.

\section{CIRCAג. Onagré. Juss.}

1 Stem ercet: L. ovate, denticulate, opaque, downy.

2 Stem ascending: cordatc, serrated, shining: cal. membranous.

9. TERONICA. Scrophularine. R. Br.

* Spikes or clusters terminal.

1 L. oppositc, bluntish, crenato-serrulated; very entire at the apcx: stem ascending, simple.

2 L. opposite, elliptic, obtuse, uncqually crenato-serrated: stem nearly erect.

3 Clustcrs clongated, many-flow: L. elliptic-lanceolate: stems erect: caps. ovate, 4 -valved.

4 Corymb. few-flow. I. clliptical: stems diffuse: caps. ovate, 4 -valved.

5 Corymb. somewhat spiked: L. ovate, smootl, slightly serrated: cal. ciliatc: stem ascending, simple.

6 Raccme somewhat spiked: L. ovate, slightly crenate, 3-nerved, smooth : caps. obcordate, shorter than the style.

** Spikes or clusters lateral.

7 L. clliptical, flat: stem crceping.

8 Racemes opposite: $\mathbf{L}$. lanccolate, serrated: stem erect.

9 Racemes alternate: fruit-stalks reflexed: L. linear, denticulate.

10 Spikes pedunc: L. opposite, rough: stem procumbent.

11 L. elliptic-lanccolatc, slightly serrated, ratlicr hairy: stems ascending: caps. abrupt, undivided.

12 IRaccmes lateral: L. ovate, sessile, rugged, inciso-serrated: stem lıairy bifariously.

13 Racemes elongated, filiform, few-flow: L. ovate, petioled, serrated: stem hairy all round.

$$
\text { *****avers solitary. }
$$

14 L. ovate, inciso-serrated, slorter than the jedune: stems procumbent: seeds pitcher-sliaped.

$15 \mathrm{~L}$. ovate, inciso-serrated; floral leaves lanceolate, longer than the pelunc: stem erect. 
ORDER I. MOVOG) MI.

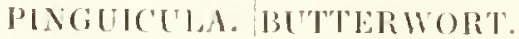

I lusitanica.... pale..........

2 vulgaris .... commun .......

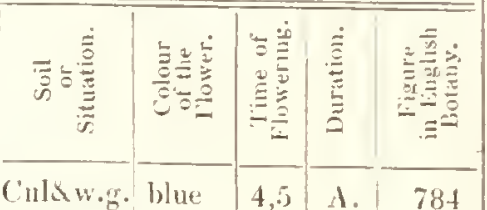

I6 hederifolia .. ivy-leaved ......C

\begin{tabular}{ll|c|c} 
san. fi.! blue & 4 & 1.
\end{tabular}

26

18 verna ..... v vernal........ Sian. fi. blue 4 S.

25

3 grandiflora ... large-foweled....

Bogs .. lilac 6.7 P.

145

liog: .. violet $\left.5,6^{\circ}\right)$ T'. 70

Ir. logs blue 5,6 P.

2184

UTRICLIAIRIA. IIOOJ)EI) MILFOII.

1 vulgaris $\ldots$. greater $\ldots \ldots . \mid$ s'tag. 1 ... yellow 6,7 1'.

253

2 intermedia .. internediate .... 4 r. logs

2489

3 minor ...... lesser ........

254

\section{LFINA.}

J) $\mathrm{L}(\mathrm{CK}-\mathrm{IIIEI).}$

I trisulca..... ivg-leaved ..... Stag. wa...... 6

2 ninor $\ldots \ldots$ lesser ......... stag.

3 gibba $\ldots . .$. gibbous $\ldots . .$. stag. wa. . . . . . 6.7

4 polyrhiza.... greater $\ldots . .$. Difches $\ldots . . .7,8$

1. 926

i. 1095

1. 1233

I. YOCOS. WATER-IIOREIIOUNO.

1 europaus .... common ...... Riv. lan. white 7,8 I

1. 2458

SAISII.

1 pratensis

CIAIRI.

2 verbenaca...

meadow ....... I ry pas.! violet $7 \quad \mathrm{P}$.

153

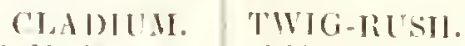

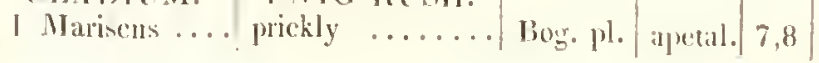

ORDER II. DIGINAA.

ANTIIOXANTIIIU. VRRNAL-GRASA.

I otloratun sweet-scentrol. 


\section{TERONIC.I.}

I6 I.. cordate, fat, 5-lohed : cal. segments cordate: sceds pitcher-shajed. I7 I.. mplement, with fingered divisions: pelunt. Ionger than the cal: seeds flat.

18 1. pimatifid, uppermost lanceolate, undivided: pedune. slorter than the cal : stem straight.

10. PIXUICLIA. I.entibularia. R. IBR.

1 Ject, obtuse, shorter than the petal : scape villous: caps. globosc.

2 Vect. cylind, acute, the length of the petal: caps. ovate.

3 Nect. cylind, acute, as long as the petal, which is reticulated; upper lip dilated: eaps, ovate.

I1. UTRICIIAIRIA. Lentibularia. R. BR.

I Nect. conical: scape fuw-flow: upper lip of the cor. the length of the palate, reflexed at the sicles.

2 Nect. conical: scape 3-flow: upper lip of the cor. flat, twice as long as the palate: L. erowded, in 3 deep-forked segments.

3 iect. keeled, very slort, obtuse, deflexed: cor. gaping, palate nearly flat.

12. J.L.MIN. Pistinceâ. Ricu.

1 L. petioled, lanceolate, proliferous.

2 1. sessile, flattish on both sides: roots solitary.

3 L. sasile, somewhat convex, hemisplerical beneatlı: roots solitary.

4 1. sessile, convex beneath: roots crowded.

1 L. sinuato-serrated.

I3. LICOPLS. Labiate. Juss.

\section{SALVIA. Labiala. Juss.}

1 L. cordate-oblong, crenate; upper embracing the stem : bracteas very small: cor, lıelmet glutinous.

2 L. serrated, sinuated, smoothish: cor. narrower than the calyx.

15. CLAI)IUII. Cuperaced. 1)E C.

I. Panicle repeatedly compound, leafy: spikes capitate: stem round:

L. prickly at the margin and keel. Schanus. Eng. Bot.

\section{ORDEL II. IOIG YNA.}

16. ANTIOXXNTIIUM. Grumined. Juss.

1 Spihe ovate-oblongr: Fl. longer than their awns, somewhat pedunc. 



\section{CLASS III.}

\section{TRIANDRIA.}

ORDER I. MONOG YNIA.

* Fl. superior.

17 I'serias. Cor. 5-cleft, gibbous at the basc. Seed 1, with a feathery radiating erown.

18 Fenil. Cor.5-eleft, protuberant at the base. Caps. crowned with the toothed calyx, without valves, of $1-3$ fertilc cells. Seeds solitary.

19 Crocts. Cor, 6-parted, equal, tube longcr than the limb. Stig. convolute.

20 Tricnosins. Cor. 6-parted, equal, tube shorter than the limb. Filam. downy. Stig. very slender, deeply divided. 21 InIs. Cor. 6-parted, alternate petals reflexed. Stig. petaliforn.

** Fl. inferior, glumose. Seed 1. (Grass-like.)

22 Schoexus. Cor. 0. Glumes clustered; outer ones barren. Style simple at the base, deciduous. Seed roundish.

23 Riricinospora. Cor. 0. Spikes few-flow. Glumes imbricated all round; outer smaller, barren. Seed beaked.

24 Crpercs. Cor.0. Glumes 2 -rowed, imbricatcd. Style simple at the base, dcciduous. Seed beardless.

25 Scirpus. Cor. 0. Glumes imbricated on every side. Style simple at the basc, deciduous.

26 Eleocharis. Cor. 0. Glumes imbricated all round. Seed crowned and articulated with the dilated hardened base of the style.

27 Erropnorva. Cor. 0. Glumesimbricated on cvery side. Seed besct with many very long hairs.

28 Ninous. Cal. 0. Cor. glume 2-valved.

Juncus 4.5. 16 .

ORDER II. DIGYNIA. (True Grasses.)

* Calynes 1-flow. ecattered.

32 lixarpa. Cal. 2-valved, truncated. Cor. 2-valved; valves uncqual, very hairy, awnless.

31 Alopeures. Cal. 2-valved. Cor. 1-valved, simple at the apex, awned at the basc. Styles combined. 
30 Pnleva. Cul.2-valved, lincar, acuminate, scssile. Cor. 2-valved, awnless, included in the calyx. Sopt loose.

29 Pialaris. Cul. 2-ralved; valves kecled, equal, including a double corolla.

33 Ponrogox. Cal. 2-valves, awned at the apex. Cor. included, outer valve awned at the apex. Seed loose.

34 Miniva. Cal. 2-valved, ventricosc, larger than the corolla. Stig. villous. Seed coated with the hardened corolla.

35 Acrostis. Cul. 2-valved; valves aeute, shorter than the corollia. Cor. membranous, hairy at the base, unchangerl. Stig. plumose. Seed loose.

36 Criodox. Cal. 2-valved; valves equal, laneeolate, shorter than the corolla. ('or. 2-valverl; valves unequal, compressed. Seed coated with the hardened corolla.

49 Spartixa. Cul.2-valved; valves laneeolate, clasping. Cor.2-valverl; valves rather unequal. Nect. 0 . Seeds loosc. Styles combined.

53 SтrрA. Cul 2-valved. Cor. awn terminal, jointed at its base.

55 L.Acurus. Cul. 2 -valved; valves fringed, with terminal feathery awns. Cor. outer glume 3-awned.

37 Digraria. Cul. 2 or 3 -valied; valves mnequal, close-pressel, awuless. C'or.2-valved; valves uncqual, depressed, awnless. Seed coatcd with the hardencel corolla. Arundo 2. 3. 4. 5. Melicu 1.

* Calyses 2, or 3-floucered, scattered, 2-valcad.

39 Airs. Flurets without a rudiment between then. ("or. unehanged. seed loose.

42 Mrica. Thudiment of a thirrl flower between the florets. Seed coated with the lardened corolla.

40 Houcus. ('or. awned; one floret barren. Cul. keeled. Seed conted witl the hardened corolla.

38 Paxicus. One floret neuter: Cor. of the perfect one awnless. Cal. ribled. Seed conted with the hardened corolla.

4l Ilikocutos: Florels 3. central one perfect, with 2 stamens; lateral ones barren, with 3. ('or. permanently membranous.

Seeds loose. Styles distinct.

43 stowna. Florets 2 or 3 , all perfeit. ('ir. outer valve tootluel; inner cloven. Seed loose. Stules combined.

*** Calyres many-flouered, scallered.

47 Burd. Con" awnless, tumid, expanded, concave, without a keel. sicel depres:ed, growing to the corolla. 
45 Por. Cor. valves ovate, rather acute, awnless. Seed loose, elliptic, oblong.

44 Grycomu. Cor. awnless, cylind. furrowed, ribbed, abrupt, not keelerl. Seed loose, eylind. oblong.

46 Triona. (ur, orbicular, obseurely ribbed, deeply eloven, witlı an intermediate point. Seed loose, depressed.

48 1).erris. Cor awned at the summit, keeled, eompressed ; inner valve folded, 2 -ribbed. Cal.compressed, taper-pointed, unequal. seed loose, oblong.

51 Frstuci. Cor. awned at the summit, or pointed; keeled, nearly eylind: inner valve flat, with 2 downy ribs. Cal. coneave, aeute, unequal. Seed loose, oblong.

50 Cryostrirs. Spikelets in pairs; one neuter. Cor. awned at the summit, keeled, eoneave; inner valve flat, 2 -ribbed. Cal. awned, equal. Seed loose, elliptie-oblong.

52 Browus. Cor. awned at the baek, eloven, eoneave; inner valve flat, with 2 bristly ribs. Seed elliptie-oblong, united to the inner valve.

54 Irisa. Cor. awned at the baek, eloven, nearly eylind; immer valve flat, ovate. Seed elliptic-oblong, united to the lard outer valve.

56 Anvino. Cor. surrounded with long permanent hairs. Flor. 1 or many.

******arers aggregute, on a jointed, or toothed, common stalk, with lateral excavations.

57 Lolium. Cal. 1-leaved, fixed, many flow.

58 liottbollia. Cal. 1-leaved, sometimes 2-parted, imperfeetly 2-flow.

$\mathrm{Fl}$. alternate, on a jointed raehis.

61 Tniтseum. Cal. 2-valved, solitary, many flowered. Rachis wavy, toothed alternately on each side.

59 lismus. Cal. 2-valved, lateral, aggtegate, with 2 or more florets.

60 llondeun. Cal.2-valved, lateral, 1-flow. growing 3-together; only the eentral flower perfeet.

ORIVR III. TRIGINIA.

62 Montra. Cal. 2-leared. Cor. 1-pet. Caps, 3-valved, 3-seeded.

64 Pozvarpon. Cal.5-leared. Pet.5. Caps.3-valved, many-seeded. 63 Hotostrus. Cal.5-leaved. Pet.5, jugged. Caps. nearly eylind. opening at the apex, with 6 teeth, many-seeded. 
CLASS III. TRIANDRIA.

ORDER I. MONOGYNA.

\begin{tabular}{l|l} 
INNEAN NAMIES. & ENGLISH NAMES. \\
VALERLANA. & VALERIAN.
\end{tabular}

1 rubra ......

2 dioica ......

3 officinalis....

red ......... old wa.

marsh .........

4 pyrenaica....

great wild ......

heart-leaved ....

Marshes

Marshes

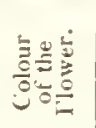

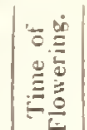

.

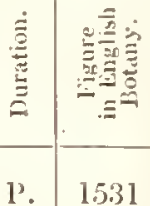

\begin{tabular}{r|r|r|r} 
rose.. & $6-9$ & $\mathbf{l}^{\prime}$ & 1531 \\
\hline & &
\end{tabular}

Sc.Wds.

'?. 628

flesh.. 6

l. $159 \mathrm{I}$

FEDIA. CORN-SALLAD

1 olitoria .......

eornmon .......

2 dentata......

oval-fruited ....

Corn fi. blue

4,6

A. $8 \mathrm{II}$

Corn fi.! pur-ish 6,7

A. $* 1370$

CROCUS.

1 sativus... saffron.........

2 vernus ....... spr

spring ..........

Meadow

Meadow

violet

$9 \mathrm{P}$

343

3 reticulatus $\$$.. net-rooted ......

Meadow bl.tyel

4 nudiflorus....

naked-flowering..

Meadow

violet

$10 \mathrm{P}$.

491

TRICIIONEMA. TRICIIONEMA.

1 Bulboeodium . channel-leaved ..

Guernsey blue

IRIS.

IRIS.

1 Pseudacorus... yel

2 fotidissima - stint

SCHONUS.

\section{BOG-RULII.}

1 nigricans ....

black ....

Moi.pla. yellow

Sha. pla. dullpur 5,6

I'.

596

RIIYNCIIOSIORA. BEAK-RUSII

1 alba Tur.bogs 


\title{
CLASE IIT. TRIANDRIA. ORDER I. MONOGYNIA.
}

\author{
SPICIFIC ClIARACTER.
}

17. TALERIANA. Talerianer. I)E C.

l Fl. monandrous, spurred: L. lanceolate, nearly entire.

2 Fl. triandrous, dioecious: L. radical ovate; stem pinnatificl.

3 Fl. triandrous: L. all pinnate: leaf. lanceolate, almost uniform.

$4 \mathrm{Fl}$. triandrous: L. of the stem eordate, serrated, petioled, the uppermost pinnate.

18. FEDIA. Vulerianea. DE C.

1 Fl. triandrous, stem dichotomous: L. linear-tongue-shaped, olstuse.

2 Fl. triandrous: stem diehotomous, with axillary flowers: L. lineartongue-slaped: fruit ovate, ribbed: crown erect.

\section{CROCUS. Iridec. Juss.}

I Stig. projecting, three-parted: segments linear.

:2 Stig. inelosed, trifid: lobes wedge-shaped, ineised: cor. tulse hairy at the mouth.

"3 Stig. inclosed, trifid: eor. three outer segments recurved: coat of the bulb strongly reticulated.

4 Stig. inclosed, trifid: with many-cleft laeiniated pencil-form lobes: Fl. leafless.

\section{TRICHONEMA. Iridea. Juss.}

I Stalk short, mostly 1-flowered: L. linear, ehannelled, recurved. Ixiu. Eng. Bot.

\section{IRIS. Iridea. Juss.}

11 Cor. beardless: inner segments less than the stigma, ereet: L. ensiform: seeds angular.

:2 Cor. heardless: inner segments greatly spreading: stem 1 -angled: L. ensiform: seeds globose.

$$
\text { ** Fl. inferior, chaffy. Seed } 1 \text {. }
$$

\section{SCIIENUS. Cyperacea. 1) E C.}

II Culm eylind. naked: head ovate: involuere 2 -leaved, one valve subulate, longer.

\section{RIIYNCHOSPORA. Cyperacea. DE C.}

Il Heads abrupt: Fl, diandrous: L. tapering: seed bristly at the base. Schanus. Eng. Bot. 


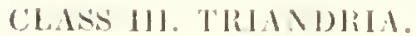

()liblik I. MONOCi)JIA.

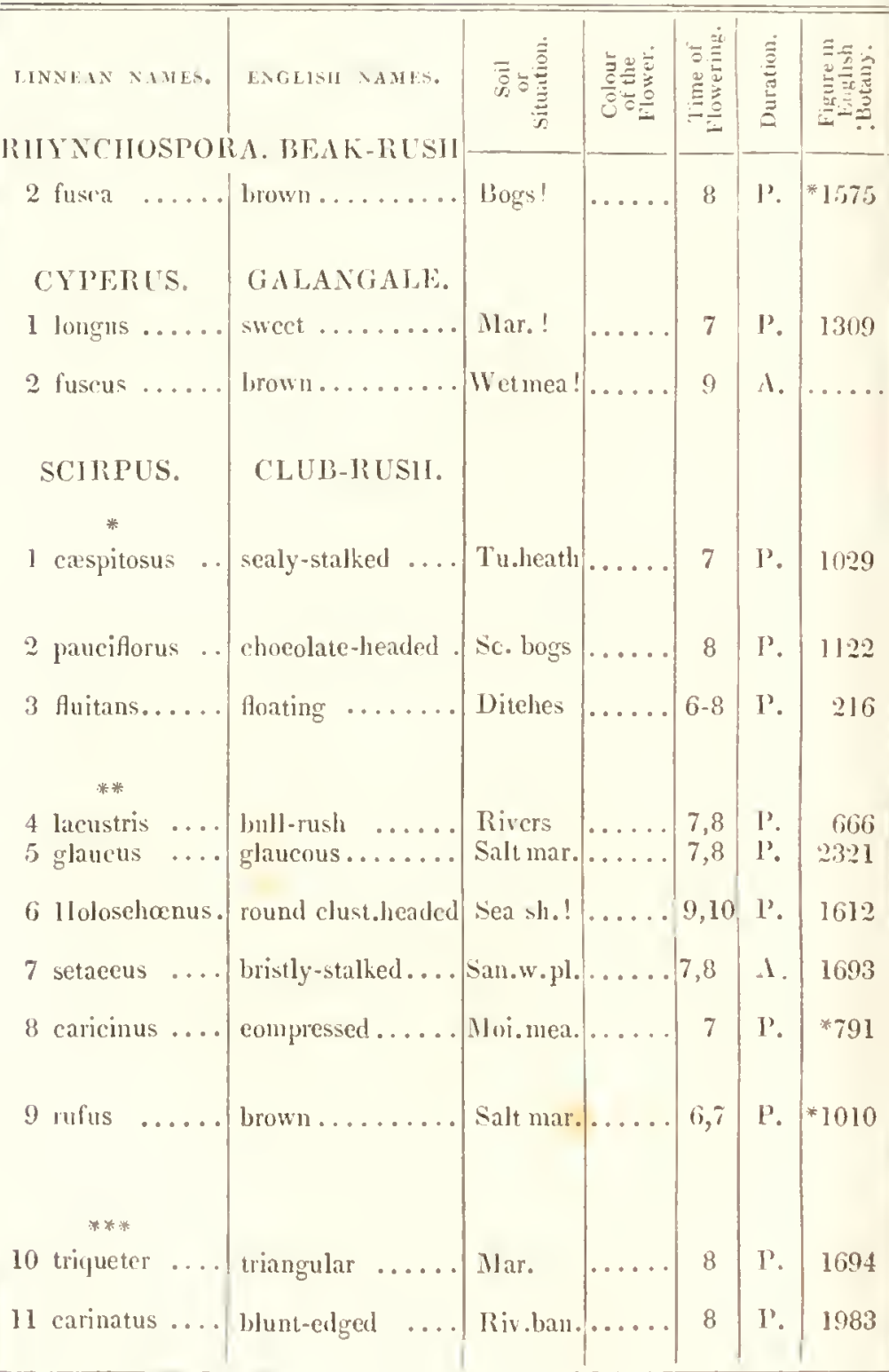




\section{SPFCIFIE CHARACTFR.}

\section{RHYNCHOSPORA.}

2 lleads ovate-oblong: Fl. triandrous: L. thread-shaped: seed with three bristles at the base. Schanus. Eng. Bot.

\section{CYPERUS. Cyperaccir DE C.}

I Culm 3-sided, leafy: umbel leafy, deeompound : pedune. nakerl : spikes alternate.

2 Culm 3-sided: umbel compound, with three unequal leaves beneath: spikes erowded, spreading every way: stig. 3.

\section{SCIRPUS. Cyperacer. DE C. \\ * Spike single.}

I Culm eylind. striated, sheathed, encompassed with numerous seales at the base: spike term. outer glumes largest, with leafy points.

2 Culm eylind. striated, sheathed at the base: spike term. few-flow. longer than the outer glumes.

3 Culm leafy, flaceid, floating: pedune. alternate, naked: spikes solitary, term. few-flow.

\section{** Spikes many: culm cylindrical.}

4 Culm naked: pan. cymose, decompound, term. spikes ovate.

5 Culm naked, glaucous: pan. eymose, not higher than the braetea: spikes ovate: stig. 2 .

6 Culm naked: heads elustered, pedune. or sessile: braeteas unequal: L. channelled: seed without bristles.

7 Culm naked, setaceous: spikes sometimes in pairs, sessile, surmounted by a leafy bractea: sced furrowed, without bristles.

8 Culm leafy at the base: spikes aggregate, 2-ranked, many-flow: L. flat, with rough edges and keel: seed 6-bristled at the base. Schonus, Eng. Bot.

9 Culm leafy at the base: spikes aggregate, 2-ranked, few-flow: L. channelled, smooth, without a keel: seed without bristles. Schanus. Eng. Bot.

$$
\text { *** Cutm 3-sided: panicle naked. }
$$

10 Culm ereet, naked, acuminate: spikes lateral, sessile, or peduneled: stig. 2. seed smooth.

11 Culm naked, triangular upwards : pan.eymose, term : bractea pungent, chanmellerl, ereet: stig. 2. 
CLASS 1H. TRIANDRIA.

ORDER I. MONOGYNIA.

ERIOPHORUM. COTTON-GRAS.

1 vaginatum ... harc's-tail

CLUB-RUSH.

12 maritimus.... salt-marsh ......

13 sylvaticus.... wood

ELEOCIIARIS. SPIKE-RUSH.

I palustris ... crecping ........ Mar.

\begin{tabular}{|c|c|c|c|c|}
\hline 点施竎 & 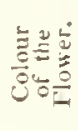 & 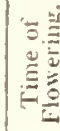 & 竎 & 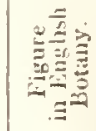 \\
\hline & & & & \\
\hline
\end{tabular}

2 multicaulis .. many-stalked ....

3 acicularis....

least

Tu. $\operatorname{logs}$

Moi.hea.

2 capitatum.... round-hcaded ....

3 alpinum .... alpine .........

Moors

3,4

P.

873

Sc.mou.

Sc. bogs

P. 2387

P. 311

***

4 polystachion.. broad-leaved .... Bogs

6,7

Marshes

5 pubescens.... downy-stalked

Bogs

7 gracilc

common

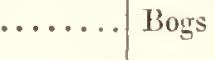

slender ........ St.mou

Se.mou. ......

7

P.

563

$P$. 4,5 ?

6 angustifolium

gracile ......

NARDUS.

MIAT-GRASS.

1 stricta common Moi.hea.

564

ORDER II. DIGYNIA.

$\begin{aligned} & \text { PIIALARIS. } \\ & 1 \text { canariensis } \$ .\end{aligned}\left|\begin{array}{l}\text { manured ........ Uncul.pl. } \\ \text {....... }\end{array}\right| 6-8 \mid$ 
SPECLFT CIARACTFR.

SCIRI'US.

***** Culm 3-sided: panicle leafy.

12 Pan. term. leafy. Spikes conglomerate: glumes torn, with an intermediate point: stig. 3 .

13 Culm leafy, eyme term: pedune. sheathed at the base, superdeconpound: spikes crowded.

26. ELEOCHARIS. Cuperaced, DE C.

I Culm round: root ereeping: stig. 2 : seed lenticular, most convex at one sille. Scirpus. Eing. Bot.

2 Culm round : root fibrous : stig. 3 : seed aeutely triangular, as well as the permanent base of the style. Scirpus. Eng. Bot.

3 Culm quadrangular: stig. 3 : seed numerously furrowed, without bristles at the base: filam. permanent. Scirpus. Eng. Bot.

27. ERIOPHORUII. Cuperaces. DE C.

* Sprike solitur'y.

1 Culms sheathed, cylind: 3 -sided at the apex: spike ovate: glumes membranous.

2 Culm sheathed, entirely cylind: spike roundish: glumes membranous.

3 Cuhn triangular, naked above the leaves, which are shorter than their sheaths: spike oblong-ovate: glumes firm, strongly keeled.

\section{*** Spikes several.}

4 Culms cylind: L. flat: stalks of the spikes smooth: hairs thrice the lengtl of the spike.

5 Culm angular upwards: L. flat: stalks of the spikes downy: hairs twice the length of the spikes.

6 Culms cylind : L. ehannelled, 3-sided at the apex: stalks of the spikes smooth : hairs four times the length of the spikes.

7 Culms compressed towards the top: L. 3-sided, ehannelled above: spikes pedunc. longer than the braetea: hairs twice the length of the spike.

28. NARDUS. Graminer. Juss.

I Spike setaeeous, straight: L. thriee the length of their sheaths.

ORDER II. DIGYNIA.

29. PIIALARIS. Graminee. Juss.

1 Pan. ovate, spike-like: eal. glumes boat-slaped, entire: outer cor. 2-valved, naked. 
CLASS III. TRIANDRIA.

ORDER II. DIGYNIA.

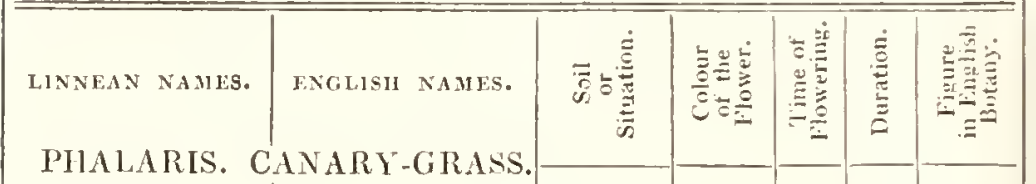
2 arundinacea. . reed...........

\section{PHLEUI. CAT'S-TAIL-GRASS.}

1 pratense .... common ....... Mea.pas......6-10

Ditches $\ldots . .787$

402

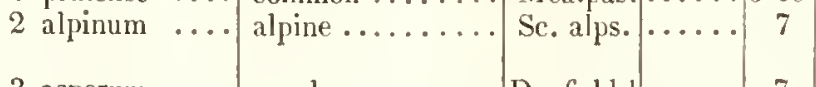

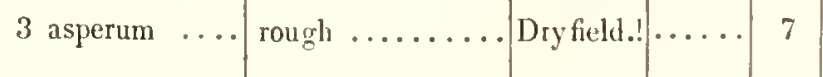

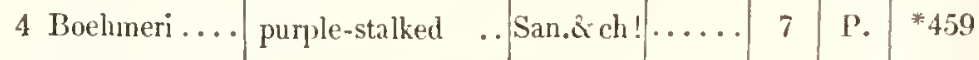

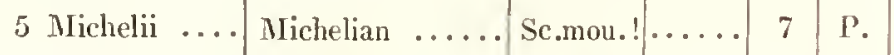

2265

6 arenarium....

Sca.......... San. fi.

ALOPECURUS. FOX-TAIL-GRASS.

1 pratensis ... meadow ....... Mead.

5,6 A. 222

2 alpinus...... a a

Sc. mou.

3 agrestis

......

alpine.........

4 bulbosus

slender

Road side

P. $\quad 1076$

P. 519

A. 1077

$$
5
$$

bulbous

. Salt mar.!

5 geniculatus .. floating

Ponds dit.

.

6 fulvus .

\section{KNAPI'A.}

KNAPPIA.

1 agrostidea....

early .........

POLYPOGON: 13FARD-GRASS.

1 monspeliensis . anmal

Salt mar.

2 littoralis perenuial. Salt mar.

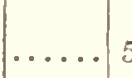

Pond:

P. 1467 
SPECIF1C CHARACTE1.

\section{PIIALARIS.}

2 Pan. upright with spreading branches: Fl. crowded, leaning one way: outer cor. 2-valved, hairy.

\section{PILEUM.}

1 Spike cylind. very long: glumes ciliate on the back, longer than the awns.

2 spike ovate-cylind: glumes ciliatc ou the back: awns the length of the glumes.

3 Pan. cylind. spike-like: glumes naked, thickening upward: culm branched. P. paniculatum. Eng. Bot.

4 Pan. nearly cylind: spike-like: glumes linear-lanceolate, their inner margin abrupt: stem simple. Phaluris. Eng. Bot.

5 Pan. nearly cylind: spike-like : glumes lanceolate, taper-pointed, hairy, fiingcd: cor. oblong, hairy : inner valve cloven.

6 Spilc ovate-lanceolate, blunt: glumes lanceolate, fringed: cor. shortened, crenated. Phalaris. Eng. Bot.

\section{ALOPECURUS.}

1 Culm erect, smooth: spike somewhat lobed: cal. glumes united at their base, villous, shorter than the awn of the corolla.

2 Culmerect, smooth : spike ovate: cal. glumes downy, obliquely abrupt, ncarly as long as the awns of the corrolla.

3 Culm erect, roughish : spike very simple, attcnuated: glumes nakedisl, unitcd at their base; keel dilated.

4 Culm erect:spike very simple, attenuated : cal. glumes separate, linear, pointed, villous: root bulbous.

5 Culm ascending, geniculate: spike somewhat lobed, cylind: glumes combined at the basc, retuse, hairy, shorter than the awn: cor. notehed.

6 Culm bent at the joints: spike somewhat lobed, cylind: glumcs combined at the base, retuse, hairy, inclosing the awn: anth. roundish.

32. KNAPPIA.

1 Agrostis minima. Fl. Brit. 82.

\section{POLYPOGON.}

$1 \Lambda$ wn much longer than the cal: root fibrous. Agrostis panicea, Ling. Bot.

2 Awn not longer than the cal: root creeping. Agrostis littoralis, Eng. Bot. 


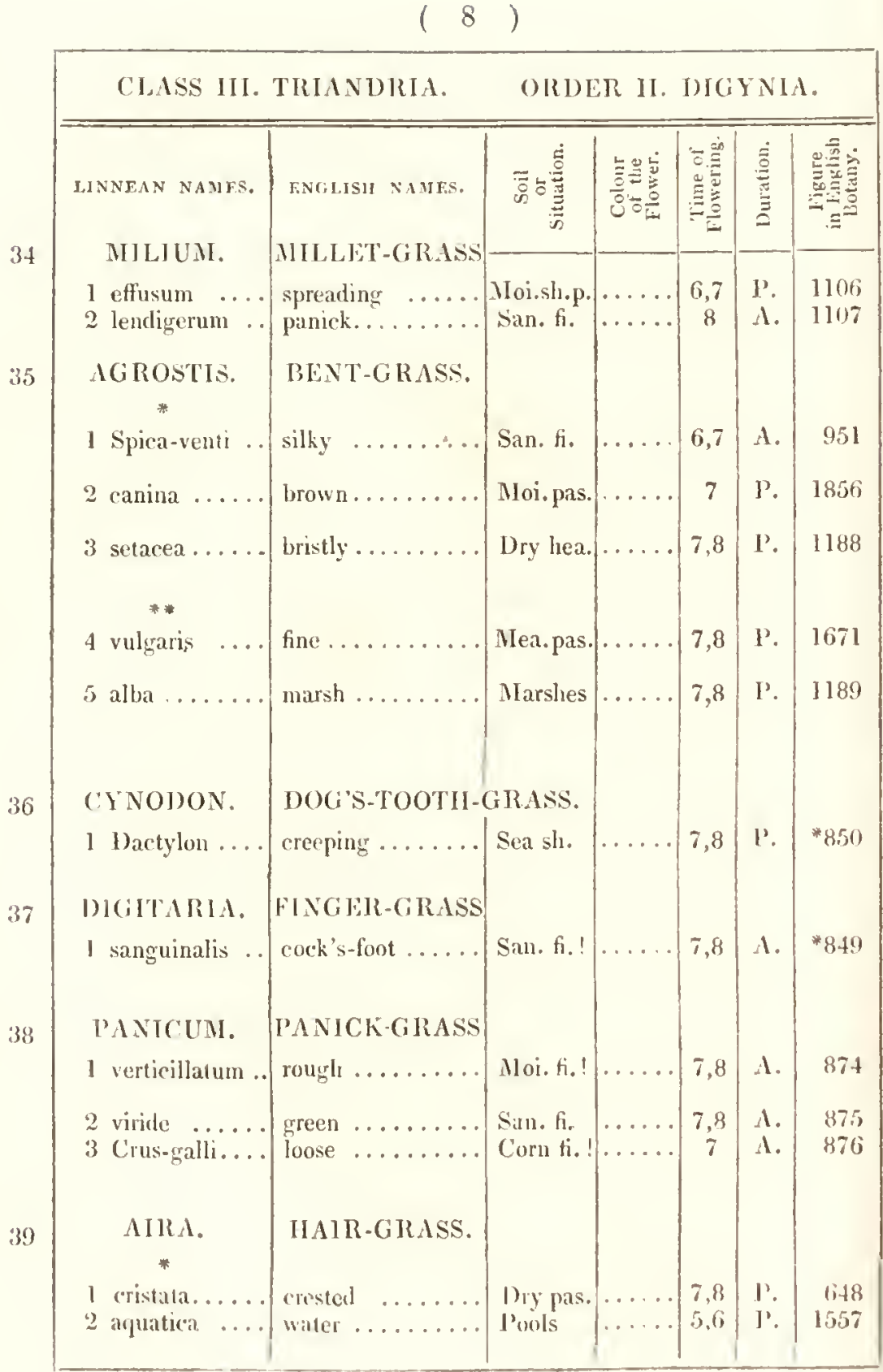


34. NIILIUM.

1 Fl. panicled, diffuse, awnlcss.

2 l'an. somewhat spiked: Fl. awned.

\section{AGROSTIS.}

* Awned.

1 Outer pet. with a very long straight stiff awn, beneath the apex: pan. spreading.

2 Cal. ovate, coloured: cor. naked: awn on thc back incurved: culms prostrate, somewhat branched: stipula lanceolate.

3 Cal. lanceolate: cor. awned at the basc: awn geniculate: L. radical setaccous: culm nearly erect: pan. close, oblong.

\section{***anless.}

4 Pan. spreading: branches divaricate, capillary: cal. equal, inner pctal twice as short, retuse : culm erect: stipula abrupt.

5 Pan. condensed at the basc of the main divisions: stalks rough: culm branched, crceping: Fl. hcaped: calyx equal, lanceolate, keel bristly: "stipula oblong, ribbed.

\section{CYNODON.}

1 Spikes 4 or 5 crowded together: cor. smooth. Panicum. Eng. Bot.

\section{DIGITARIA.}

$1 \mathrm{~L}$. somewhat hairy, as well as their sheaths: $\mathrm{Fl}$. in pairs: cal, larger valve rough at the edges. Janicum. Eng. Bot.

\section{PANICUM.}

1. Pan. spiked, verticillatc: spikelets in $4 \mathrm{~s}$ : several bristles, rough with reversed teeth.

2 Pan. spiked, cylind. with prominent bristles, rough with erect teeth.

3 I'an. crect, branched, bristly: Fl. awned, unilateral: L. lanceolate, harsh, naked, without stipulæ: rachis mostly 5 -angled.

$$
\begin{aligned}
& \text { 39. AIRA. } \\
& \text { * Awnless. }
\end{aligned}
$$

1 Pan. spiked: cal. longer than the pedunc: glumes all pointed.

2 Pan. spreading: Fl. awnlcss, smooth, obtuse, longer than thc calyx: I.. flat: stipula oblong. 
CLASS IH. 'TRHANDRIA.

ORDER H. DIGINIA.

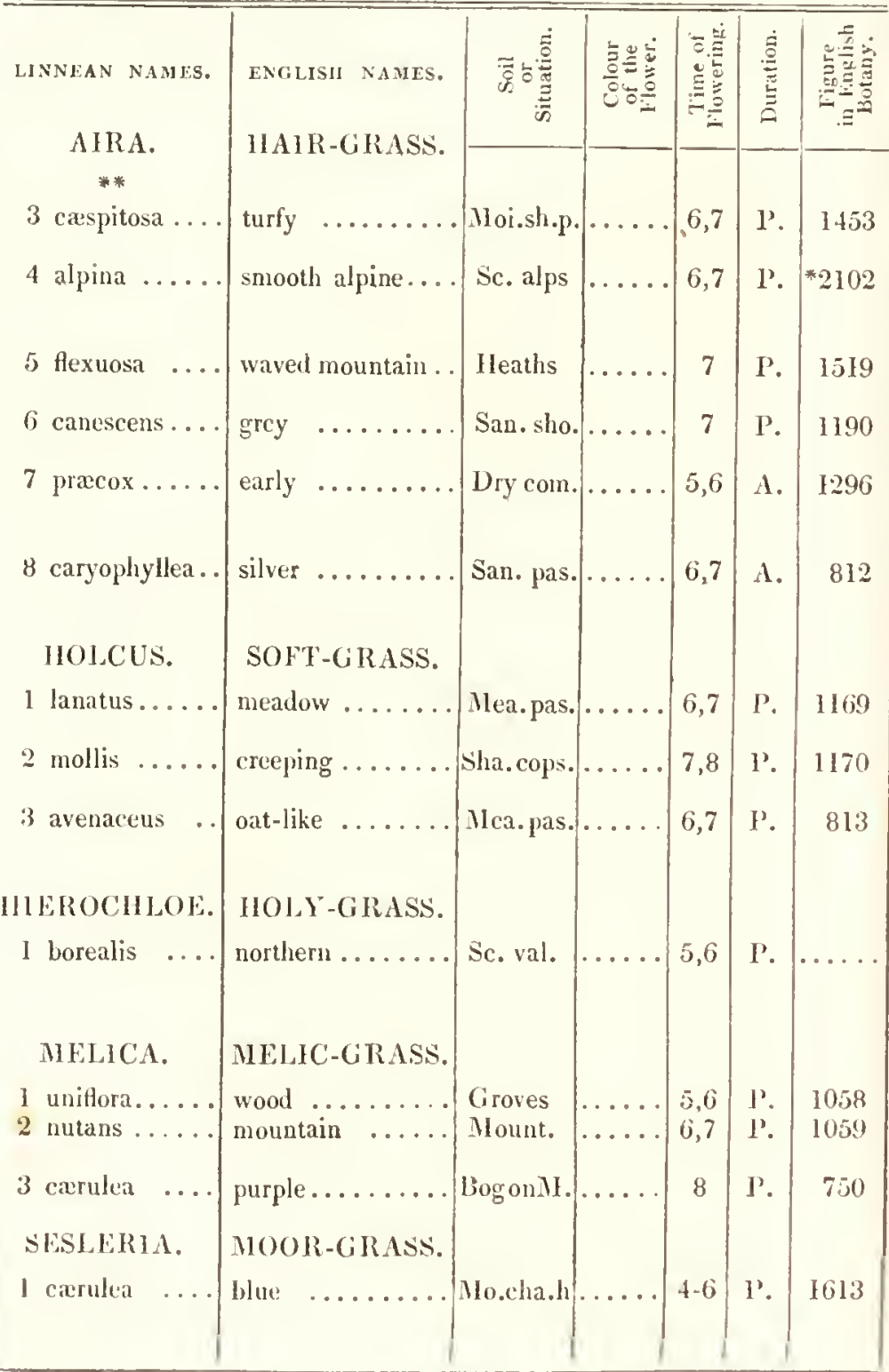




\section{SPFCIFIC CHARACTER.}

\section{AIRA.}

* Awned.

3 L. flat: pan. sprcading: Fl. villous at the base, one of them on a hairy stalk: awn short, from the base of the outer valve.

4 L. involute, with very smooth sheaths: pan. close: Fl. awned, villous at the base, onc on a smooth stalk: awn short, from near the top of the outer valve. A. livigata. Eng. Bot.

5 L. setaccous: culm ahmost naked: pan. spreading, trichotomous: pedunc, flexuose: awn from the niddle of the outer valve, twisted.

6 L. setaceous: culm leafy: pan. compact: awns clubbed at the apcx, not longer than the calyx, hairy at the joint.

7 L. setaceous: sheaths angular: pan. close, erect: flor. length of the calyx, both sessile; awn nearly twice as long, from the base of the valve.

8 L. setaceous : sheaths close: pan. trichotomous, divaricate: flor. sessile; awn twice as long, from above the middle of the valve.

\section{HOLCUS.}

1 Glumes villous: hermaph. floret awnless: male with a bowed-recurved awn: L. downy on both sides: root fibrous.

2 Gilumes nearly equal, almost naked : inferior hermaph. floret awnlcss: male with a geniculate awn: root creeping.

3 Glumes unequal, smonth: hermaph. floret superior, scarcely awned: male with a geniculate refracted awn: L. lather harsh: root knotty.

\section{IIIEROCIILOE.}

1 Pan. somewhat unilateral: pedunc. smooth: perfect flor. awnless; barren ones slightly awned. Nect. 2-parted: seg. unequal, linear, L. Hat.

\section{MELICA.}

1 Pan. 1-rowed, branched: Fl.crect: spikelet with only one perfect foret.

2 Pan. compact, 1-rowed, almost simple: Fl. pcndulous: spikelet with two perfect florets.

3 Pet. acutc: pan. compact: Fl. erect, cylindrical.

\section{SESLERIA.}

I spike ovate-oblong, imbricated: bractca alternate: outcr valve of the cor. 3-toothed. 
CLASS III. TRIANDRIA.

ORDER II. DIGINIA.

\begin{tabular}{|c|c|c|c|c|c|c|}
\hline LINNEAN NAMES. & ENGLISII NAMES. & 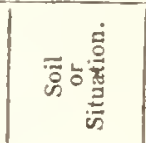 & 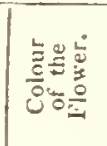 & 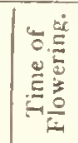 & 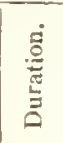 & 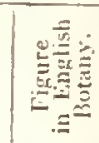 \\
\hline $\begin{array}{l}\text { GLYCERIA. } \\
\text { I aquatica ... }\end{array}$ & $\begin{array}{l}\text { SWEET-GRASS. } \\
\text { reedy .......... }\end{array}$ & Ditehes & & 7 & P. & $* 1315$ \\
\hline 2 fluitans...... & floating ....... & Pools & & 6,8 & P. & $* 1520$ \\
\hline 3 distans ..... & reflexed ........ & San. pl. & & 7,8 & P. & *986 \\
\hline 4 maritima .... & ereeping sea .... & Sea sl.. & & $7-10$ & P. & $* 1140$ \\
\hline 5 procumbens.. & procumbent sea .. & Sea sh. & & 7,8 & A. & $* 532$ \\
\hline 6 rigida $\ldots \ldots$ & liard ... & Wall.bks. & & 6 & A. & $* 1371$ \\
\hline $\begin{array}{c}\text { POA. } \\
1 \text { compressa }\end{array}$ & $\begin{array}{l}\text { MEADOW-GRA } \\
\text { flat-stalked ...... }\end{array}$ & Walls & & $6-8$ & P. & 365 \\
\hline 2 alpina ..... & alpine .......... & Se. alps. & & 7,8 & P. & 1003 \\
\hline 3 laxa ....... & wavy $\ldots \ldots \ldots$ & Se. alps & & 7 & P. & $* 1123$ \\
\hline 4 bulbosa .... & bulloous ........ & San. pl. & & $4-6$ & P. & 1071 \\
\hline 5 trivialis..... & rouglish ........ & Mea.pas. & & $6-10$ & P. & 1072 \\
\hline 6 pratensis ... & smooth-stalked .. & Mea.pas. & & 5,6 & P. & 1073 \\
\hline 7 annua $\ldots .$. & annual $\ldots . .$. & Past. & & $3-11$ & A. & 1141 \\
\hline 8 glauca ...... & glaueous.... & Mount. & & 6,7 & P. & 1720 \\
\hline 9 nemoralis.... & wood $\ldots . . . \ldots$ & Woods & & 6 & $\mathrm{P}$ & 1265 \\
\hline $\begin{array}{l}\text { 'l'RJOIDIA. } \\
1 \text { decumbens }\end{array}$ & $\left\{\begin{array}{l}\text { HLATII-GRASS. } \\
\text { decumbent ...... }\end{array}\right.$ & Spo. bogs & & 7 & P. & $* 792$ \\
\hline
\end{tabular}




\section{GLYCERI $\Lambda$.}

I Pan. ereet, branehed, spreading: Fl. many, oltuse, 7-ribbed: Nect. cloven, acute. Por. Fing. Bot.

2 P'an. ollong, branched, spreading: spikelets close-pressed: Fl. many, olstuse, 7 -ribbed, with short intermediate ribs at the base. Nect. obtuse, tumid. Por. Eng. Bot.

3 Pan. branched, spreading: branehes finally reflexed: Fl. about 5: obtuse, 5-ribbed, polished: root fibrous. Poa. king. Bot .

4 P'an. branched, close; creet after flowering. Fl. about five: somewhat pointed, 5-ribbed: root creeping. T'oa. Eing. Bot.

5 Pan. laneeolate, unilateral, 2-ranked, close, with rough stalks: the main one cylind. Fl. about five: bluntish, 5 -ribbed. Poa. Fing. Rot.

6 P'an. lanceolate, unilateral, close, 2 -ranked, with smooth stalks; the main one bordered: Fl. about seven: acute, searcely ribbed. Pou ling. Bot.

\section{POA.}

I Pan. I-rowed, compaet: culm aseending, compressed: florets angular, united at their base by a web: root creeping.

2 Pan. difluse: spikelet 4-flow, cordate: glumes ovate, rather falcate, free: lower stipula very short.

3 Pan. flexuose: spikelet 3-flow: glumes ovate, united at their base by a web: stipulæ all lanceolate. P. flexuosu. Eng. Bot.

4 Pan. sliglitly flexuose: spikel. 4-flow: Fl. hairy at the keel, united by a web: leaves serrulated: eulm bulbous at the base.

5 Pan. diffuse : spikel. 3-flow: glumes lanceolate, 5-nerved, united at their base by a wel : stipulæ elongated : root fibrous.

6 P'an. diffuse: spikel. 4-flow: glumes lanceolate, 5-nerved, united at their base by a web: stipula short, obtuse: root creeping.

7 Pan. divaricate: spikel. ovate, 5-flow: flor. rather remote, 5-nerved, free; eulm obliopue, eompressed.

8 Pan. spreading: spikel. ovate, about 3-flow. glumes bluntish, silky at the keel, and lateral ribs, villous at the base: stipular very short.

9 Pan. and 1. attenuated: spikel. lanceolate, about 3-flow: glumes adute, indistinctly 5 -nerved; silky at the keel, and lateral ribs: stipula very short, notched.

\section{TIRIODI $\Lambda$.}

1 l'an. wearly simple, close, erect: Fl. 4. their middle tooth shortest: calyz-glumes smootlı: stipula lairy. l'ot. Ling. Bot. 


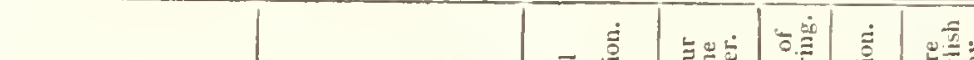

LINNEAN NAMES.

NAMES.

BRIZA. QUAKING-GRASS.

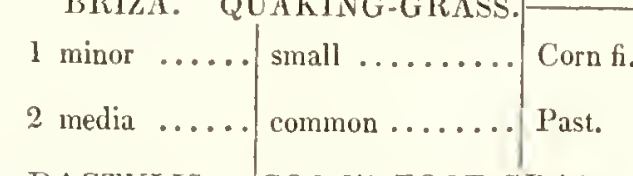

DACTYLIS. COCK'S-FOOT-GRASS.

1 glomerata... rough ......... Mead.

6-8 I'.

335

SPARTINA. CORD-GRASS.

1 stricta $\ldots . .$. twin-spiked.....

Sal. mar.

CYNOSURUS. DOG'S-TAIL-GRASS.

1 cristatus .... c c

2 cchinatus $\ldots . .6$ r

crested ........ Past.

FESTUCA. FESCUE-GRASS

l ovina ...... shcep's Dis

2 vivipara .... viviparous .....

3 duriuscula .. hard .........

4 rubra

. crecping ........

5 bromoides....

6 Myurus .... wall...........

San. gr.

7 uniglumis.... single-husked ....

8 gigantca .... gigantic ........

9 calamaria.... recd-like ........

10 loliacea..... 
SPECIFLC CIARACTER.

\section{BRTZA.}

1 Spikel. triangular, 7-flow: cal. longer than the flor: stipula lanceolate, very long.

2 Spikel.ovatc, 7 -flow : cal. shorter than the flor : st ipula very short, obtusc.

\section{DACTYLIS.}

1 Pan. distantly branched: Fl. in globular tufts, unilateral : eor. awncl 5-ribbed, tapcr-pointed.

\section{SPARTINA.}

3 Spikes 2 or 3 erect, with smooth stalks: glumes downy, outer valve sinallest. Dactulis. Eng. Bot.

\section{CYNOSURUS.}

1 Neutcr spikelets awnless: spike simple, linear.

2 Neuter spikelets awned: spike compound, ovatc.

\section{FESTUCA.}

1 Pan. 1-rowed, compact: flor. roundish, smooth at the base: eulm square: L. folded, setaceous, rough.

2 Pan. 1-rowed, compact: flor. compressed, kccled, awnless, somewhat downy, as well as the ealyx: culm, square: L.folded, setaceous, smooth.

3 Tan. 1-rowed, diffuse: flor. awned: eulm cylind: stem leaves flat: root fibrous.

4 Pan. l-rowed. flor. roundish, awned: L. downy above, more or less involute: root creeping.

5 l'an. l-rowed, erect: flor. sulsulate, awned, rough at the apcx: L. sctaceous, shorter than the sheath: upper half of the culm nakcd.

6 Pan. 1-rowed, elongated, nodding: flor, subulate, awned, rough at the apex: L. awl-sliaped, somewhat keeled, very short. Culm leafy to the top.

7 Pan. 1-rowed, erect, nearly simple: flor. subulate, eompressed, awned: cal. l-valve very short.

8 Pan. nodding, branched, lax, decompound : flor. 3-6 ovate laneenlatc, awned : 1. ensiform, nerved: stipula auricled, stem-clasping.

9 Pan. 1-rowed, erect, muel branched, compact: flor'. oblong, angular, awnless, inner valve folded in the middle: L. ensiform, striated.

10 Spike 2-rowed, nodding: spikel. subsessile, linear-oblong: flor. cylind. nervcless, awnless, with 5 slight ribs at the top. 
CLASS III. TRIANIRIA.

ORDES II. DIGYNLA.

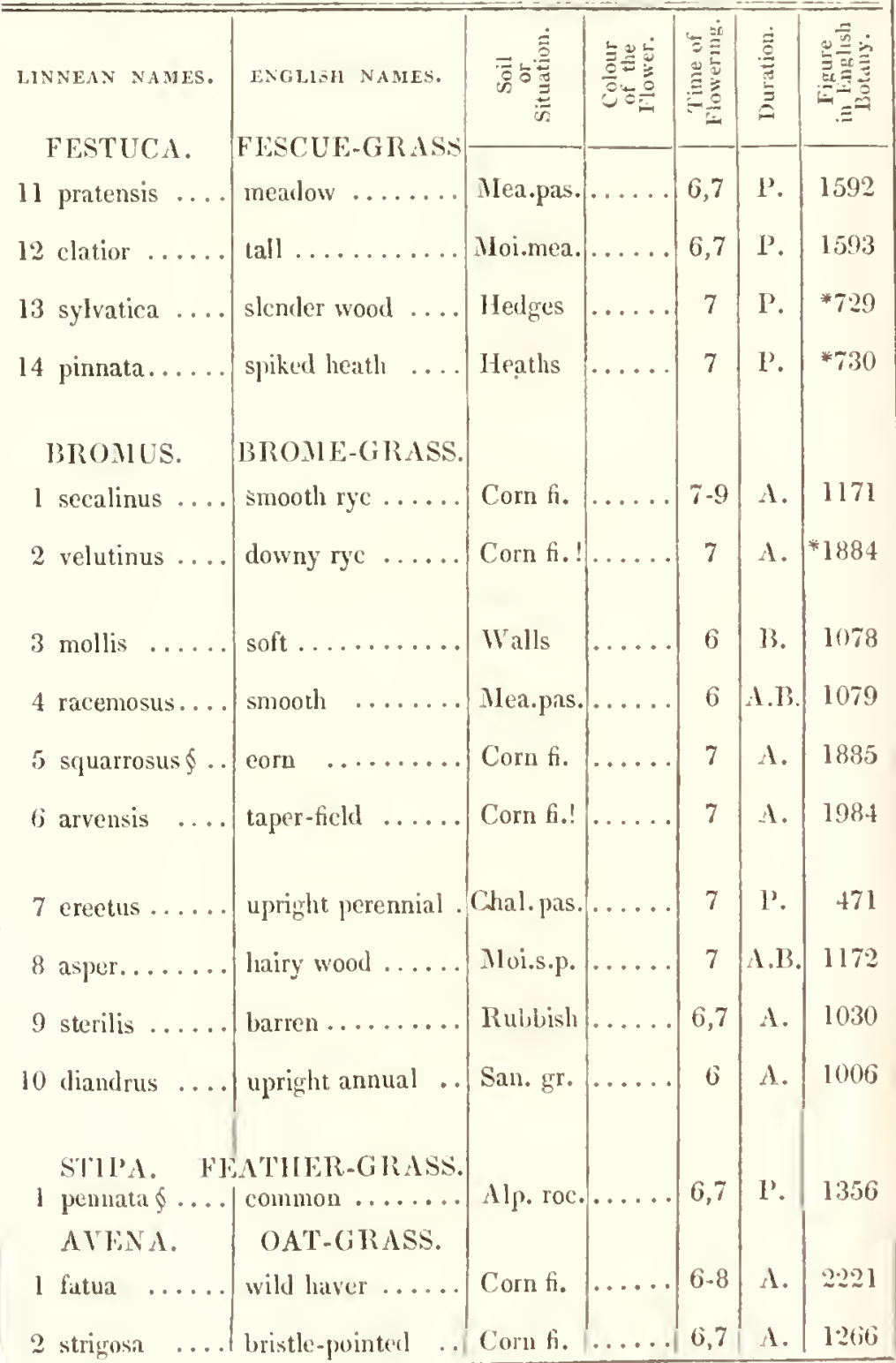




\section{FESTUCA.}

11 Pan. 1-rowed, rather upright, lax: spikel. lincar, compressed, rather obtuse: flor.cylind. indistinctly nerved : nect. 4-cleft . root filsrous.

12 Pan.sululiffuse, nodding, much branched, lax : spikel.ovate-lanccolate, acute: flor. cylind. ind istinctly nerved : nect. 4-cleft : root cree ping.

13 spike simple, nodding, 1-rowcd: spikel. sessilc, roundish: awns longer than the glume: L. hairy: root fibrous. Bromnes. Fing. Bot. 14 Spike simple, erect, 2-rowed: spikel. sessile, roundislı: awns shortcr than the glume: L. nakedish : root ereeping. Bromus. Eng. Bot.

\section{BROMUS.}

1 Pan. spreading: pedunc. mostly simple: spikel. orate, 10-flow: flor, distinct, roundish: awns wavy: L. slightly hairy.

2 Pan. spreading: pedunc. nearly simple: spikel. ovate-lanceolate, 10-15-flow: Hor. somcwliat imbricated, roundish, downy: L. slightly lairy. B. mulliflorus. Eng. Bot.

3 Pan.erect, compact: pclunc. branched: spikel. ovate: flor. imbricatel, depressed, nerved, downy: L. and sheaths soft and downy.

4 Pan. rathcr erect, diffuse: pedune. simple: spikel. ovate, 6-thow: flor. imbricated, depresscd, nerved, smooth: L. somewhat downy.

5 Pan. nodding: pedunc. simple: spikel, ovate, 12 -flow: for. imbricated, depressed: awns divaricate: L. downy.

$6 \mathrm{Pan}$. spreading: pedune. branehed drooping, half-whorled: spikel. lanccolate, 8-flow: flor. elliptical, imbricated, depressed, with 2 elose ribs on eaeh side.

7 Pan. ereet: pedunc. gencrally simple : flor. 8 . loosely imbricated, lanecolate: L. radical, very narrow, with hairy ciliæ.

8 l'an. nodding, branched: for. 8. lanceolate, roundish, almost nerveless, downy: L. uniform; lower hirsute.

9 Pan. nodding, generally simple: floret 7. laneeolate, nerved, furrowed: 1. downy.

10 Pan. ercet, spreading, searcely branehed: flor. laneeolate, nerved, furrowed, diandrous.

I Awns feathery.

\section{STIPA.}

\section{AVENA.}

1 Panicled: spikelets pendulous: cal. about 3-flow: flor. all awned, hairy at the base, with an oblique scar: nerveless.

2 pan. oblong, 1-rowed: eal. 2 -flow: flor, tipperl with a double bristle. 
CLASS III. TRIANDRIA.

ORDFIR I1. DIGYNIA.

\begin{tabular}{|c|c|c|c|c|c|c|}
\hline IINNEAN RAMES. & ENGILISI NAULS. & 司告高 & 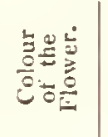 & 它蓄 & & 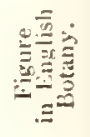 \\
\hline 3 pubescens... & $\begin{array}{c}\text { OAT-GRA.S. } \\
\text { downy........... }\end{array}$ & Clıal. pas. & & 6 & P. & 1640 \\
\hline 4 pratensis .... & narrow lcaved... & Chal.pas. & & 7 & P. & 1204 \\
\hline 5 alpina ..... & great alpine $\ldots$. & Se. alps & & 7 & P. & 2141 \\
\hline 6 flavcscens... & yellow......... & Mca.pas. & & 6,7 & P. & 952 \\
\hline $\begin{array}{l}\text { LAGURUS. } \\
1 \text { ovatus } \ldots . .\end{array}$ & $\begin{array}{l}\text { II ARES-TAIL-G } \\
\text { oval spiked ....... }\end{array}$ & $\begin{array}{l}\text { RASS. } \\
\text { Guernsey }\end{array}$ & & 6 & A. & 1334 \\
\hline $\begin{array}{l}\text { ARUNDO. } \\
\text { l Phragmites .. }\end{array}$ & $\begin{array}{c}\text { REED. } \\
\text { common ........ }\end{array}$ & Ditehes & & 7 & P. & 401 \\
\hline 2 epigejos .... & wood...$\ldots \ldots$ & Moi.wo.! & & 7 & I. & 403 \\
\hline 3 Calamagrostis. & small ... & Moi. wo. & & 6,7 & P. & 2159 \\
\hline 4 stricta.... & smallest close... & Sc. mar. & & 6 & $P$. & 2160 \\
\hline 5 arenarià $\ldots$ & sea $\ldots \ldots \ldots \ldots$ & Sea coa. & & 7 & P. & 520 \\
\hline LOLHUM. & DARNEI. & & & 6 & & \\
\hline $\begin{array}{l}1 \text { perenne .... } \\
2 \text { temulentum . }\end{array}$ & perennial ... & $\begin{array}{l}\text { Mea.pas. } \\
\text { Corm fi. }\end{array}$ & & $\begin{array}{l}0 \\
7\end{array}$ & A. & $\begin{array}{l}010 \\
1124\end{array}$ \\
\hline 3 arvense...... & white...$\ldots \ldots$ & Corn fi. & & 7 & $\Lambda$. & 1125 \\
\hline $\begin{array}{l}\text { ROTBOLLTA. } \\
1 \text { ineurvata ... }\end{array}$ & $\begin{array}{l}\text { II } \Lambda R D-G R A S S . \\
\text { sea } \ldots \ldots \ldots \ldots\end{array}$ & Sen coa. & & 8 & ג. & 760 \\
\hline $\begin{array}{c}\text { ELlils. } \\
1 \text { arenarius .... }\end{array}$ & $\begin{array}{l}\text { LYMH-GRASS. } \\
\text { upright sea ...... }\end{array}$ & Sca coa. & & 7 & I'. & 1672 \\
\hline 2 genieulatus .. & pendulous ...... & Sea sh.!! & & 7 & r. & 1586 \\
\hline
\end{tabular}




\section{A VENA.}

3 Pan. generally simple, ereet: eal. about 3-flow: partial stalks bearled: L. Hat, downy: root somewhat creeping.

4 Spikc erect: cal. ahout 5-How: partial stalks hairy: L. involute, serrulated, naked; sheaths smooth.

5 Pan. erect: eal. about 5-How: partial stalks bearded under eacin: L. serrulated, naked; shcaths rough : culm eompressed.

6 Pan. much branched, lax: eal. about 3-flow. unecpual: partial stalks hairy: L. flat, somewhat pubesecnt.

1 Spike ovate.

\section{IAGURUS.}

\section{ARUNDO.}

1 Cal. 5-flow: paniele lax.

2 Cal. 1-flow. longer than the eor: pan. crect: Fl. imbrieated, crowled, 1-rowed: cor. awned at the back: L. lanceolate.

3 Cal. 1 -flow. longer than the cor: pan, ereet, diffusc: Fl. scattered, awn term. short: L. linear.

4 Cal. 1-flow, pointed, equal with the eor: pan. ereet, close: cor. awned at the baek.

5 Cal. 1-flow. longer than the cor: pan. spiked: Fl. erect, slightly awned: L. involute, pungent.

\section{LOLIUM.}

1 Cor. slightly awned: spikel. longer than the cal: flor. laneeolate.

2 Awns longer than the eorolla: spikelet shorter than the calyx: flor. elliptical: culm rough on the upper part.

3 Spike searccly awned: spikel. the length of the calyx: Fl. elliptical: eulm very smooth.

\section{ROTBOLLIA.}

i Spikc cylind. subulate: cal. glumes combined below: flor, solitary: cor. awnless.

\section{ELYMUS.}

I Spike ereet, close: eal. laneeolate, the length of the spikelet: L. with a spinous point.

2 Spike bent downwards, lax; main stalk not winged: cal. setaccous, spreading, longer than the spikelet: $\mathbf{L}$. with a spinous point. 
CLASS III. TRIANIORIA.

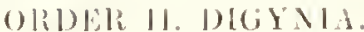

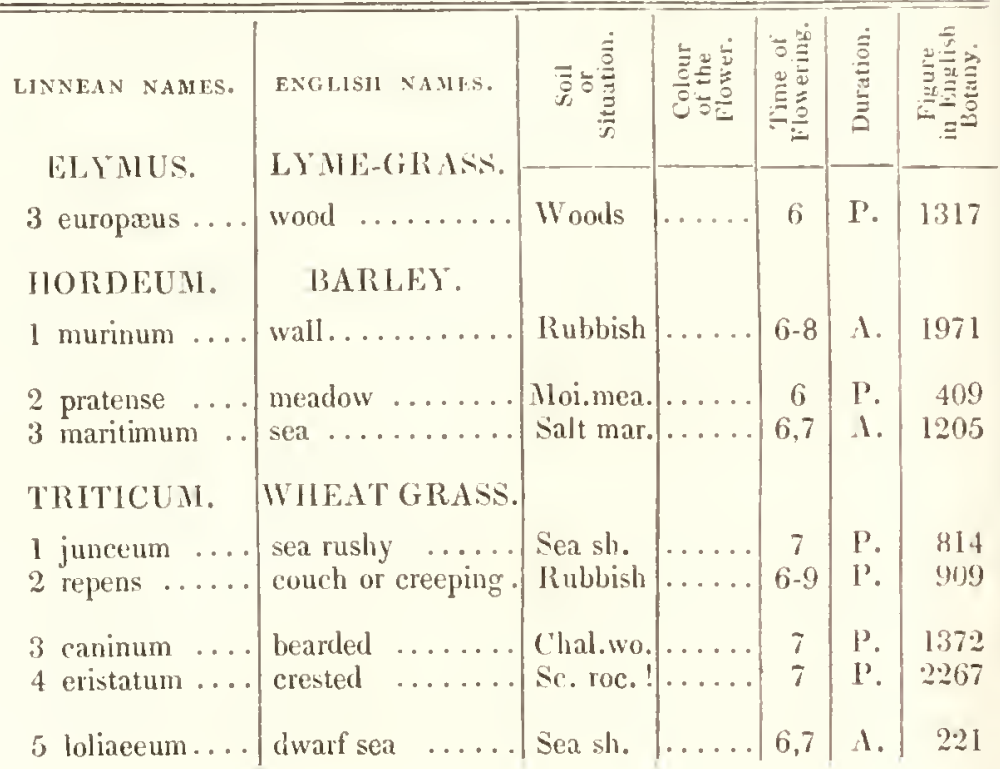

()RDER III. 'TRIGYNIA.

63

\section{MONTIA.}

l fontana......

BLINKS.

Spring

whitc

11OLOSTEUM. JAGGFI) CHICKWEED).

1 umbcllatum.. umbclliferous... 
SWFCIFIR FHARACTFR.

ELYMUS.

3 Spike erect; main stalk wingcd: spikel. 2-flow, awned: invol. without streaks.

\section{HORDEUM.}

1 Flor. lateral male, awned: calyx glumes of the intermediate one lanreolate, fringed.

2 Flor, lateral male, with shorter awns: cal. glumes all setaceous, rough. 3 Flor. lateral male, with shorter awns; their inner calyx glume semi-ovate.

\section{TRITICUM.}

1 Cal. blunt, 5-How: L. involute, with a spinous point: root crecping.

2 Cal. lanceolate, many-nerved, 5-flow: Hor, sharp-pointed: L. Hat: root creeping.

3 Cal. pointed, about 5-nerved, 4-flow : flor. awned : L. flat : root fibrous.

4 Cal, elliptical, awned, keeled: flor. awned: spikel. 2-rowed, close: culm simple.

5 Cal. olbtuse, many-flow: spike simple, 1-rowed: flor. awnless: culm branched: root fibrous.

\section{ORDER III. TRIGYNIA.}

62. MONTIA. Portulucer, Juss.

1 Much branched, smooth: L. opposite, spatulate, entire.

63. HOLOSTEUM. Caryophyller. Juss.

1 Fl. in umbels: stam. various in number: I. ovatc, acute.

64. POLICARPON. Illecelrer. R. Br.

I Much branched: L. opposite, often in double pairs. 


\section{CLASS IV.}

\section{TETRANDRIA.}

\section{ORDER I. MONOGYNTA.}

* Fl. 1-pet. 1-seeded, superior.

65 Dipsacus. Cal. common many-leaved; proper superior, 1-leaved, eup-shaped, erowning the seed.

66 Scabiosa. Cal.common many-leaved; properdouble, superior, erowning the seed.

**Fl. 1-pet. 2-seeded, superior.

70 Rußia. Cor. eampanulate. Fruit pulpy.

69 Galiun. Cor. flat. Fruit dry.

68 Aspinula. Corr. tubular. Fruit without a erown.

67 Sinerardia. Cor. tubular. Fruit erowned with the ealyx. Seed 3-toothed.

**** Fl. 1-pet. many-seeded, inferior.

71 Exacum. Cor. salver-sliaped. Stam. shorter than the limb. Caps. 2-eelled, opening at the apex.

72 Plantago. Cor. refraeted. Caps. 2 or 4 -eelled, eut round. Stum. very long.

73 Centunculus. Cor. tubular. Caps.1-eelled, eut round. Stam.inelosed.

\section{Some Gentiunte.}

***** F1.4-petalled.

75 Erimenum. Nect. 4, ineumbent on the petals, saek-like. Pont many-seeded, superior, 1-eelled. Cal. eadueous.

76 Connus. Nect. 0 . Drmpe an inferior 2-eelled nut. Cardamine, 2. Senebiera, 2.

*********io apetalous.

77 Panetaria. Cal. 4-eleft, inferior. Seed eovered with the clongated ealyx. Stum. elastic. Fl. some female, whose ealyx remains unchanged.

78 Issanns. Cal. 4-eleft, superior. Caps. quadrangular, t-celled, erowned with the calys.

74 Saxguisoras. Cal. 4-eleft, superior, eol. Stum. dilated upwards. Caps. quadrangular, l-eelled, not bursting.

79 Alenemuld. Cal. 8-eleft. Seed 1-2. nakert. 
80 Burrosis. Cor. 4-pet. Caps. 2-valved, 2-seeded.

Alchemilla. Gentianse. Cuscute.

ORDER 1II. TETRAGYNIA.

81 ILEx. Cor. 1-4-pet.wheel-shaped. Berry4-seeded. Style.0. Fl. some male.

85 Moexcuia. l'et. 4. Caps. I-celled, I-valved, 8-toothed. Cal. 4-leaved.

84 Samixa. Pet. 4. Caps. 1-celled. Cal.4-leaved.

87 lianiola. l'et. 4. Caps.8-celled, 8-valved. Cal. many-cleft.

86 Tillea. P'et. 3-5. C'aps. many, many-seeded.

82 Potamogeton. Pet. 4. Cal.0. Seed 4, naked, sessile.

83 Rupria. Pet. 0. Cal.0. Seed 4, pedicelled. 
CLASS IV. TETRANDRIA.

ORIER I. MONOGYNIA.

(1)

SIERARDIA. FIELD MADDER.

1 succisa .......

1 fullonum \& .. fullers.........

2 sylvestris ....

wild

3 pilosus ...... small

TEAsEl.

2 arvensis ....

devil's bit ......

3 columbaria ..

ficld.

(

\begin{tabular}{|c|c|c|c|c|}
\hline 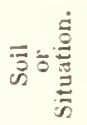 & 当 & 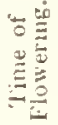 & & 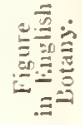 \\
\hline & & & & \\
\hline
\end{tabular}

Hedges purple 7 B. 2080

\begin{tabular}{ll|l|l} 
Moi.hed. purple 7 & b. & 1032
\end{tabular}

Moi. pla. white 8,9 B.

877

smal

Dry pas.

\begin{tabular}{c|c|c|c|c} 
Past. .. & violet & $8-10$ & P. & 878 \\
Corn fi. & purple & 7 & P. & 659
\end{tabular}

\begin{tabular}{c|c|c|c|c} 
Past. .. & violet & $8-10$ & P. & 878 \\
Corn fi. & purple & 7 & P. & 659
\end{tabular}

\begin{tabular}{c|c|c|c|c} 
Past. .. & violet & $8-10$ & P. & 878 \\
Corn fi. & purple & 7 & P. & 659
\end{tabular}

\begin{tabular}{c|c|c|c|c} 
Past. .. & violet & $8-10$ & P. & 878 \\
Corn fi. & purple & 7 & P. & 659
\end{tabular}

1 arvensic a... blue a............

Corn fi. blue 6-8 A.

891

\section{ASPERULA.}

WOODRUFF.

1 odorata......

sweet .........

2 cynanchica.

small

Wools

Chal, so. bluslı

6.8

I.

1311

G MLIUN.

BEI)-STRAIV.

1 cruciatum....

2 palustre $\ldots .$.

cross-wor

white

3 Witheringii ..

4 saxatile

$$
\text { s }
$$

5 uliginosun ..

6 erectun ....

Rourterater....

smooth heath...

Heaths

rough mars

upright marsh...

7 cinercum ....

8 aristatum.... grey

bearded .
Bushy pl. yellow 5 P'.

143

Moi. mea. white

7

I’. 1857

l'. 2206

Ileaths white

7

l'.

815

Marsles whita

Moi. pas. white

Scotland white

sic. hills white 
SPECIFIC CHARACTER.

\section{DIPSACUS. Dipsacer. Juss.}

1 L. connate, serrated: chaff recurved: invol. rcflexed.

2 L. opposite, serrated: chaff straight: invol. inflexed, longer than the head.

3 L. petiolerl, appendiculate: invol. deflexed, equal to the head.

\section{SCABIOSA. Dipsucer. Juss.}

1 Cor. 4-cleft equal: L. stem toothed : heads almost globular.

2 Cor. 4-cleft radiating: L. pinnatifid, incised : stcm rough.

3 Cor. 5 -cleft radiating: I. ladical ovate or lyrate, crenate:"stcm lcaves pinnatifid: segm. linear.

\section{SIIERARDIA. Rubiacer. Juss.}

l L. all whorled: Fl. terminal.

\section{ASPERULA. Rubiacer. Juss.}

1 L. eight together, lanceolate: Fl. in tufts, peduncled: fruit bristly.

$2 \mathrm{~L}$. linear, four together; upper very unequal: Fl. all 4-cleft: fruit smooth.

\section{GildUM. Rubiacer. Juss.}

* Fruit smooth.

1 1. in fours, ovatc, hairy: stem simple above, hairy: pedunc, axillary, 2-leaved.

2 L. in fours, obovate, unequal, obtuse: stcms diffuse, branclied above.

$3 \mathrm{~L}$. in fives, spreading, lanceolate, awned, ciliate: stem slightly branched, upright, rough with reversed hooks.

$4 \mathrm{~L}$. in sixes, obovate, olstuse, mucronate: stem much branchcd, prostrate, smooth: fruit granulated.

5 L. in sixes, lanceolate, edges rough, like the stem, with reversed prickles; inueronate, stiff; cor. larger than the fruit.

6 L. alsout 8-together, lanceolate, aculeato-serrulated forward, mucronate - stem flaceid, slightly hairy under cach joint. Fr. smooth and even: cor. taper-pointed.

7 L. 6-8 together, linear, mucronate, aculeato-serrulated forwards. Stem weak, much branched, smooth: Fr. sulooth : cor. taper-pointerl.

8 I. fi together, stalkerl, lanceolate, flat, reticulated, mucronate, with minute narginal prickles: stem branched, smooth: secls snooth, killney-shaped, separated : cor. taper-pointed. 
CLASS IV. TETRANDRIA.

ORIDER 1. MONOGYNIA.

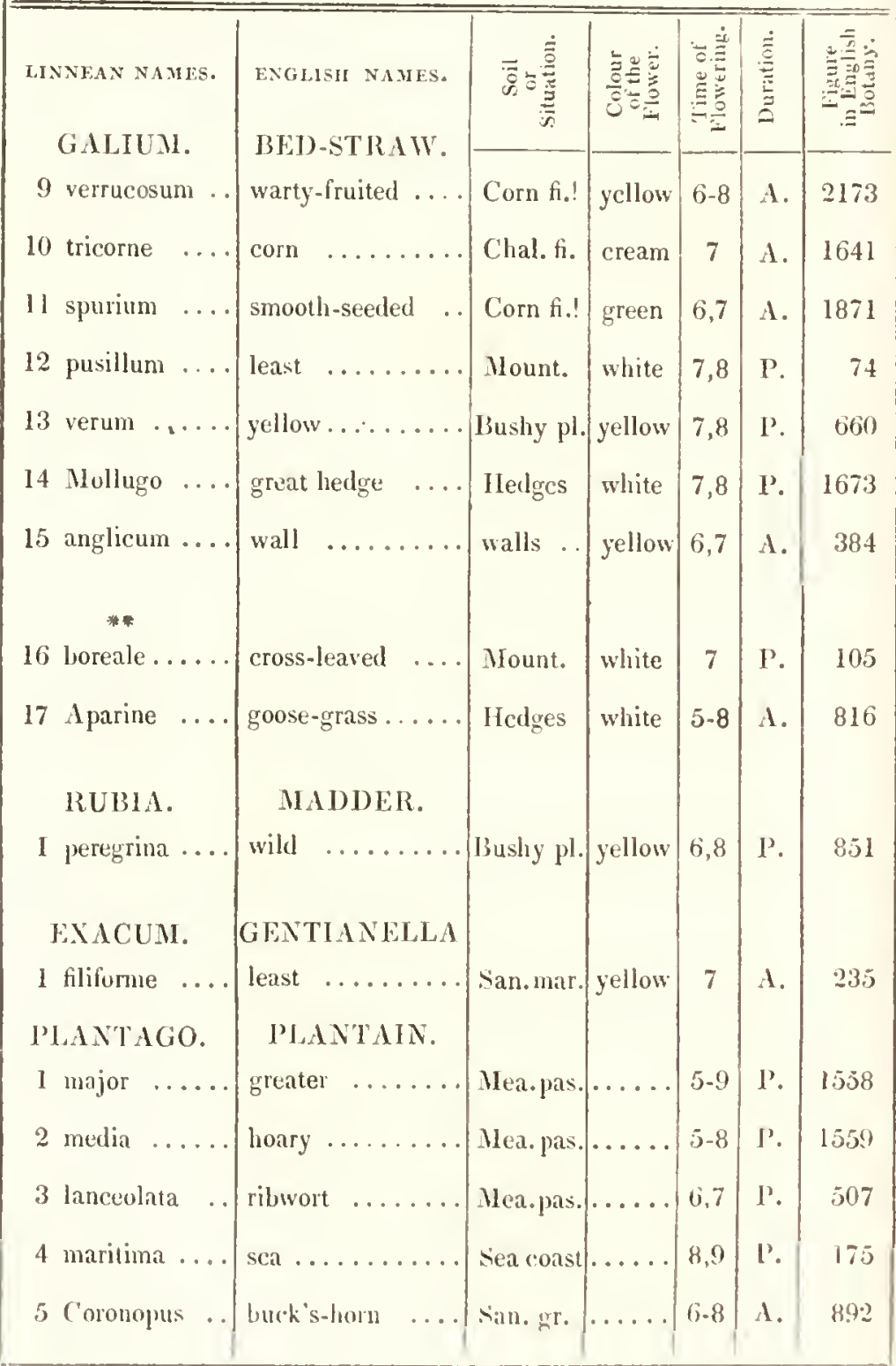




\section{G ALIUNI.}

9 L. in sixes, lanceolate, with marginal prickles pointing forwark: perdune. axillary, 3 -flow : fruit warty, drooping.

0 L. about 8-together, lanceolate; margin and stcm aculeate baekwards : pedunc. axillary, 3-flow: fruit granulated, nodding.

1 L. about 8-together; margin and stem priekled baekward: pedune. axillary, eymose: fruit smooth, ereet.

2 L.8-together, linear-laneeolate, mueronate, very entire, subpubescent: pedune. dichot: fruit very smooth.

3 L. 8-together, linear, furrowed, very entire, rough: Fl. panicled, heaped: Fr. smooth.

4 L. 8-together, elliptieal, rather obtuse, mucronate, margin rough : Fl. panieled, divarieate: cor. thiek-tipped : seeds smooth.

5 L. about 6-together, laneeolate, mueronate, margin and stem rough: pedunc. bifid: fruit granulated.

\section{** Fruit hispid.}

$6 \mathrm{~L}$. in fours, lanceolate, 3 -nerved, smooth: stem ereet : Fr. rough with hooked bristles.

7 L. 8-together, lanceolate, keeled, rough : priekles pointing baekwards: stem flaecid: Fr. bristly.

\section{RÜBIA. Rubiacer. Juss.}

1 L. generally in fours, elliptieal, shining and smooth on their upper surface: Fl. 5-cleft.

\section{EXICUM. Gentumer. Juss.}

1 L. sessile: stem filiform diehot: pedunc, elongated.

\section{I'LANTAGO. Plantagener. Juss.}

1 L. ovate, smoothish, shorter than the petiole: seape eylind: spike with imbrieated flow: seeds numerous.

2 L. ovate, pubesecnt, longer than the petioles: scape eylind: spike cylind: seeds solitary.

3 L. lanceolate, acute at each end, woolly at the base: spike ovate, naked: scape angular.

4 L. linear, mostly entire, eliannelled, woolly at the base: spike cylind. scape cylind.

5 L. linear, piunato-dentate: seape cylind. 
CLASS IV. TETRANDRIA.

ORDER I. MONOGYNA.

LINNEAN NAMES. $\mid$ INGLISH NAMES.

CINTUNCULUS CHAFF-WEED.

I minimus ....

74 SANGUISORB $\mathrm{A}$

I officinalis ....

2 media ......

small ...........

Moi.s.he.

-

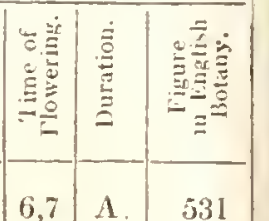

EPIMEDIUM.

I alpinum ....

CORNUS.

1 sanguinea....

2 Suecica.....

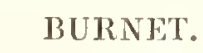

great ..........

oblong.......... S

BARRENWORT

alpine

M.thick! blood

CORNEL.

wild........... Chal. so. white

M.al.pas. purple

dwatf ..........

PARIETARIA.

PELLITORY OF THE WALL.

1 officinalis....

common

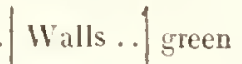

$6-9$ P.

879

ISNIIRDIA.

ISNARDIA.

1 palustris marsh.

Ponds... green

ALCHEMILLA. LADIES'MANTLE.

I vulgaris $\ldots .$. common ........ Dry pas. green

2 alpinat ..... alpine........ Riocks . green

3 arvensis .... field ......... sian. fi. green

ORDER II. DIGINIA.

BUFFONIA. IBUFFONIA.

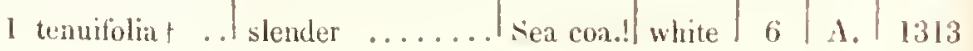

ORDER HI. THTRAGYNIA.

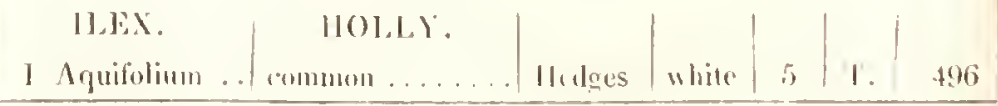


SPECIFIC CIIARACTER.

73. CLNTUNCULUS. Primularer. VENT.

1 L. ovate: Fl. axillary, sessile.

74. SANGUISORBA. Rusacer. Juss.

1 Spikes ovate.

2 spikes eylindrieal.

75. EPIMEDIUM. Berberider. D: ('.

1 L. twiee ternate.

76. CORNUS. Caprifoliacea. Juss.

1 Branches ereet: L. ovate, green on both sides: cymes naked, flat.

2 Herbaceous: branches in pairs: umb. axillary, peluneled, involuered: nerves of the leaves almost all separate.

77. PARIETARIA. Urticer. Juss.

1 L. laneeolate-ovate, 1-nerved from the base: segm. of the invol. 7. ovate, 3 -flowered: stem rather upright.

78. ISNARIDIN.

1 llerb floating: stems bluntly 4 -angled: L. ovate, entire: Fl. axillary, solitary, sessile.

79. ALCHENILLA. Rouacere. Juss.

1 L. lohed, plaited.

2 L. digitate, serrated, silky lseneath.

3 1. flat, trilobate, ineised.

\section{ORDER II. DIGYNIA.}

80. BUFFON1. Caryolingllea, Juss.

1 L. opposite, awl-shaped.

\section{ORDER 111. TEITAGNNA.}

81. ILEX, Hirinea. Jinong.

I L. ovate, acute, spinous, aml wavy: lit. axillary, somewhat eymose. 
CLASS IV. TETRANDRIA. ORDER III. TETRAGINIA.

LINNEAN NAMES. ENGLISII NAMES.

POTAMOGETON. POND.WEED.

1 natans ..... broad-leaved .... Rivers. .

2 heterophyllum various-leaved....

3 perfoliatuın ...

4 derfiatum ... perfoliate........

5 fluitans.. .6 close-lcaved $\cdots$...

Ditches

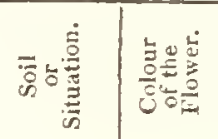

\begin{tabular}{|c|c|}
\hline \\
\hline & \\
\hline \\
\hline & 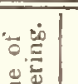 \\
\hline
\end{tabular}

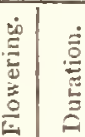

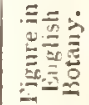

6 lucens ...... long-leaved......

Rivers. .

Ditches

Ditclies reir

purple

7,8

aldisl

6

$P$.

\begin{tabular}{l|c|l|l} 
green & 7 & $P$ & 1822 \\
green & $7-9$ & P. & 1285
\end{tabular}

7 lanecolatum.. laneeolate ......

Ditches

green

$6,7 \mathrm{P}$.

376

Wel.lake olive

7,8

I'.

1985

8 crispun .... curled ......... Thir

Rivulets reddish

6,7

P. 1012

9 compressum..

Rivulets

10 cuspidatum ..

flat-stalked ....

Por

11 gramineum .. grassy .........

Ponds . .

grcen

6,7

$P$.

418

P?

Ditches

green

7

I'.

2253

12 pusillum ....

small

Ditehes

13 peetinatum .. fennel-leaved ....

Ditehes

green

RUPIIA.

l maritima

RUPIIA. SAGINA.

1 procumbens...

...

Sal. dit.

$7-9$

I'?

136

2 maritima .... PEARL-WORT. procumbent ....

3 apctala..... annual small fiow.

Rubbish white

5-8

I'.

880

sea coa.

5-8

A.

2195

Rubbish white $5,6 \Lambda$.

881

\section{MOENCHIA.}

MOENHIA.

1 creetis

MONCHIA.

$$
\text { TILLASA. }
$$

THLl, EA.

I museos

Gra

Grav.pa. white

1. $* 609$

RADIOTA. mossy......... śan. hea. pellu. 


\section{POTAMOGETON. Fluviales. Vent.}

l L. upper ollong-ovate, petioled, floating; lower-linear, sessile.

2 L. inferior membran. linear-lanceolate, sessile: floating ones elliptical, petioled: pedunc. clavated.

3 L. cordate, embracing the stem, all immersed.

4 L. ovate, acuminate, opposite, crowded: stem dichot: spike 4-flow.

5 L. inferior lanceolate, acute, membran: upper ones elliptic-lanceolate, somewhat coriaceous.

$6 \mathrm{~L}$. ovate-lanceolate, that, attcnuated into petioles, repeatedly 3 -ribbed : spike many-flow. compact.

7 L. lanceolate; membranous, entire, tapering at the base: spike ovate, dense, few flow.

8 L. lanccolate, altcrnate, undulated, scirated; upper opposite.

9 L. linear, oltuse: stem compressed.

$10 \mathrm{~L}$. linear, obliquely pointed; many-ribbed; 3 principal ribs; all distinct: stem compressed.

11 L. linear-lanceolate, alternate, scssile, broader than the stipula: stem cylind. forked: pedunc. from the forks: scarcely longer than the spikes.

12 L. linear, opposite or altcrnate, much narrower than the stipula, spreading from the bave: stem cylind: pedune, axillary; many timcs longer than the spikes.

13 L. setaceous, parallel, app)roxinate, 2 -rowed, sheathing at the base: spikes interrupted.

1 L. setaceous, immersed.

\section{RUPJIA. Fluviales, Vist.}

\section{SAGINA. Caryophylloc. Juss.}

1 Stems procumbent, smooth: pctals very short.

2 Stems almost crect, divaricated, smooth: L. obtuse, without bristles: petals obsolete.

3 Stems almost upright, puloscent : petals indistinet: L. bristly.

\section{MiENCllid. Caryophyller. Juss.}

1 Stem erect, mostly 1 -flow: cal. leaflets acute: petals entire. Sagina. Eing. Bot.

86. 'TILJAEA. Crassulaced', DE C.

1 Stems procumbent: Fl. sessile, mostly 3 -clcft.

I Stem dichotomous: J., ovatc.

87. RADIOLA. Liner. DE C. 



\section{CLASS V.}

\section{PENTANDRIA.}

ORIER I. NONOGNNA.

\section{* H. 1-pet. inferior, 2-4-secded. (Rough leaved.)}

97 Frnus. Cor. throat naked, irregular. Stigr. 2-parted.

92 l'v.mosinu. Cor. throat naked, fumel-shaped. Cal. prismatic, 5-cleft.

89 Litnosperuer. Cor.throat naked, funnel-sliaped. Cal. 5-parted.

93 sумритим. Cor. closed with awl-shaped converging valves; limb lesll-shaped.

94 Bomago. Cor. closed with awl-shaped or notched valves; wheclshaped.

96 Lrcopsis. Cor. closad with concave obtuse valves, funnel-shaped, with a doulbly-bent tube.

95 Ispervin. Cor. closed with concave obtuse valves, salver-shaped. Cal. of the fruit compressed, lamella sinuated.

91 C'vociossum. Cor. half closed with rounded valves, funnel-sliaped. Seed depressed, imperforate, fixed laterally.

90 Arrutess. Cor. closed with eoncave obtuse valves, funnel-sliaped, tube straight, tumid below. seed hollowed out at the base.

88 Mrosoris. Cor. half closed with rounded valves, salver-shaped: lobes olstuse. Seeds perforated at the bast, borne by the calyx.

* Fl. 1-pet. inferior, secols capsular.

03 Axasulss. Caps. 1-celled, cut round. Cor. whecl-shaped, Stam. hairy.

02 Lysinaciua. Caps. 1-celled, 10-valved, glolose. Cor. whicelshaped.

99 Crolamin. C'aps. 1 celled, pulpy within. Cor. wheel-shaped, reflexed. Srim. acute.

98 Prumua. Caps. I-celled, mouth 10-eleft. Cor. salver-slaped, throat pervious, tule cylind. Stig. globose.

01 llotrous. Caps. 1-celled, 5-toothel. Cor. salver-shaped. Stum. standing on the throat. Stig.globose. Cal.5-parted.

(J0 Mexranturs. Cups. I-celled. Cor, hairy. Stig. 2-cleft.

114 1)atiri. Caps. 2-celled, 4-valved. Cor. funnel-shaped. Cal. decidnous.

115 Irosorauts. Caps. 2-relled, covered with a lid. Cor. fumelshaped. Stig. capitate. 
113 Verbasevu. Cups. 2-celled. Cor. wheel-shaped, irregular. Stig. simple. Stum. declining, bearded.

118 Eryturax. Caps. 2-celled. Cor. salver-shaped. Anth. becoming spiral.

105 Convolvulus. Caps. 2 or 3-celled. Seed 2. Cor. bell-shaped, plaited. Stig. 2.

106 Pormuonum. Caps. 3-celled. Cor. 5-parted, tube closed by 5 valves. Stau. letween the valves.

104 Azalea. Caps. 5-celled. Cor. bell-shaped. Stum. from the receptacle. Stig. capitate. Anth. with 2 pores.

128 VInca. Follicles 2, ercet. Cor. salver-shaped, twisted. Seed simple.

117 Solanua. Berry 2-celled. Cur. wheel-shaped. Anth. with 2 pores.

116 \твора. Berry 2-celled. Cor. bell-shaped. Stım. distant, illcurved. Anth, cordate.

$$
\text { * } F \text {. 1-pet. superior. }
$$

119 Samozus. Caps. 1-celled, with 5 recurved valves. Cor funnelshaped, 5-cleft, with intermediate scales.

109 Jastone. Caps. imperfectly 2-celled, opening at the apex. Cin: wheel-shaped, 5-parted. Stig. cluly-shaped. Anth. united at the base.

108 Pirterma. Caps. 2 or 3 -celled, bursting latcrally. Cor, wheelsliaped, 5-parted. Stig. 2 or 3 -clefi.

110 Lobelas. Caps. 2 or 3-celled. Cor. irregular, slit. Stig. capitate, hairy.

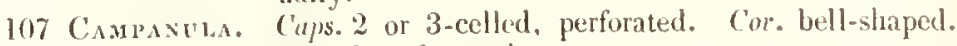
Stig. 3-cleft, revolute.

120 Lonicrina. Berry of 1 or more cells, many-seedcd. Cor. irregular. Rubia 1.

*** Fl. 4 or 5-pet. inferior.

121 Ronunus. Berry 2-4-celled. Cai. funnel-shaped, bearing the eorolla.

122 livonsuts. Caps. 4 or 5 -celled. Seed in a pulpy covering. Cal. flat. 111 Inptriens. C'aps. 5-celled, elastic. ('al. 2-leaved. (or. irrcinular. 112 Viol.A. Caps. 1-celled, 3-valved. Cul. 5-leaved. Coro irregular, spurred behind.

$$
\text { ****** Fl. 5-pet. snperim. }
$$

123 Risis. Rerry many-seeded. Cal. bearing the eorolla. Style 2-cleft. 124 11sners. Berry 3-5-seeted, surounded by the calyx. Style simjite. P'et. broad at the base. 
******* $1 . \%$. incomplete.

126 (il.mx. Cips. 5-seeded, superior. C'al. coloured, I-leaved.

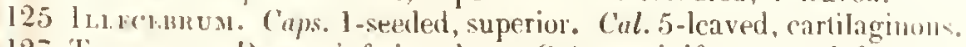

127 Thrsuvs. Drmpa. inferior, dry. ('al. staminifernus, 5-cleft.

()RDLER II. DIGYNAS.

* Fl. 1-pet. injerim.

1135 Simrin. Caps. 1-celled. Cor. wheel-shaped, with 2 nertariferous pores at the base of each scgmen.

136 Gentiana. Caps. 1-celled. Com.tulular at the base, destitute of nectariferous pores.

134 Ciss'11. Caps. 2-celled. Cor. bell-shaped.

$$
\begin{gathered}
\text { ** F1. 5-pret. inferior. } \\
\text { Stuphylen } 1 . \\
\text { ** Apetul. seeds solitury. }
\end{gathered}
$$

I30 Curvopony. Seed lenticular, tunicated, superior.

132 SAdsol... Caps. closed, imberded in a fleshy calyx. Seed with a spiral embryo.

31 BET 4. Seed reniform, immersed in a fleshy calyx.

29 HELNAMA. Caps. closed, membranous, invested with the calyx. Stum. with 5 imperfect intermediate filaments.

33 U1,3l's. ('ap)s. closed, membranous, compresed, bordered, superior. rolygumum 1.

**** Fi. 5-per. superion, 2-seeded. Umbelliferous.

A. Fruit a single an double globe.

53 Comannum. Cal. broad, unequal. Pet. radiant. Floral recept.0. $F r$. smootl, wilhout rib.

\section{Irnit beakel.}

44 sicasisis. Cial. 0). Pet. unequal, undivided. Fr. bristly: benk much longer than the seerls. $1 \%$ recept. 5-lobed, col.

13 ANommeres. Cal. 0 . P'et. equal, obcordate. Fr. scattered witl prominent bristles: beak slouter than the seeds. $1 \%$ recept. slight1ly hordered.

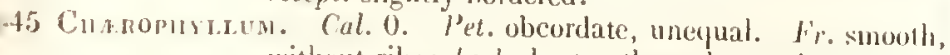
without rils: beuk shorter than the seeds, angular. Fl. recept. wavy. 


\section{Frrit solid, priclly, withont a beak.}

137 Errigum. Cal. pointed. Pet. oblong, equal, inflexed, undivided. Fl. aggregate. Fr. ovate, with straight bristles. Com. recept. scaly.

138 Saxicula. Cal. acute. Pet. lanceolate, inflexed, nearly equal. Fl. separated, dissimilar. Fr. ovate, with hooked bristles.

139 Echinophora. C'al. spinous. Pet. obcordate, unequal. I\%. separated. Fr. ovate, imbedded in the enlarged armed recept. Seed solitary.

140 Ddicus. Cul.obsolete. Pet. obcordate, unequal. Fl. separated. Fr. elliptic-oblong, compressed transversely. Seeds with 4 lows of flat prickles, and rough intermediate ribs.

141 Cauratis. Cal. broad, acute, unequal. Pet. obcorclate, unequal. Fl. imperfectly separated. Fr. elliptic-oblong, compressed transversely. Seeds with 4 rows of ascending, aw-shaped, hooker prickles; tlue interstices prickly or rough.

149 Sison. Cal. obsolete or blunt. Pet. elliptical or olycordate, with an involute point. Fl. uniform, uniterl. Fl. recept. 0 . Siules short, thick. Fr. orate, ribbed.

150 Cicuta. Cal. broad, acute. Pet. ovate, or cordate. Fl. uniform, united. Fl. recept. depressed, withering. Fr. nearly orbicular, coldate at the base, with 6 double ribs.

152 Cnsius. Cal. obsolete. Pet.obcordate. Fl. united. Fl.recept. dilated, depressed, wavy, permanent. Stules a little tunid at the base. Fr. ovate, 10-ribied, wary wlien uniije.

156 Smyrsium. Cal. small, acute. Pet. lanceolated, incurved;-or obcordate. Fl. partly barren. Fl. recept. 0. Stules tumid and depiessed at the base. Fr. broader than long, sides concave, back6-ribhed, intersticrs convex.

157 Apıus. Cal. obsolete. Pet. roundish, with an inflexed point. Fl. united. Fl. recent. thin, orbicular, wayy. Stules much swelled at the base. $F$. roundish, with 6 dorsal ribs, interstices flat.

158 Egopontus. Cal.0. Pet. obcordate, broad. Fl. united, all perfect, slightly ratiant. H. recept. 0. Stules unate at the base. Fr.elliptic-oblong, witl equidistant ribs; interstices flattisl.

162 II:3. Cal.0. Let. ubovate, with an inflexed point. Fl. united, 
all perfect. Fl. recept. 0. Styles tumid at the base, short, recurverl. Fr. elliptic-oblong, with equidistant ribs; interstices flattisl.

163 Carum. Cal. small, acute, often obsolete. Pet.obcordate. Fl. separated. Fl. recept. ammular, thin, wavy, permanent. Styles tumid at the base. Fr. elliptic-oblong, with cquidistant ribs; interstices convex.

166 Cxinum. Cal. 0. Pet. obovate, or obcordate. Fl. imperfectly separated. F\% recept. annular, thin, wavy, erect; afterwards depressed. Styles hemispherical at the base; subsequently elongated, spreading, cylind. Fr. ovate, with equidistant ribs; intersticcs deep, concave; juncture contracted.

1167 Bepifurus. Cal.0. Pet. wedge-slaped, short, involute. $\mathrm{Fl}$. all perfect. Fl. recept. 0. Styles short, broad and tumid at the base. Fr. ovate, obtuse, with acute, abrupt ribs; interstices flat; juncture contracted.

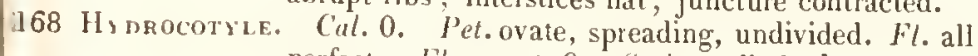
perfect. Fl. recept. 0 . Styles cylind. short, tumid at the base. Fr, nearly orbicular, angular, compressed; juncture very narrow.

142 Tosit.s. Cal. short, broad, acute. P'et. obcordate. Fl. united. Fr. ovate. Seeds ribless, scattered with prominent, ascending, rigid prickles.

\section{Murrhis 1.}

D. Fruit solid, neurly round, unarmed, without wings.

46 Mrrans. ('al. 0. Pet. obcordate, rather unequal. Fl. imperfectly separated. Fl. recept.0. Fr.deeply furrowed.

47 Busir. C'al. small, acute, unequal. P'et. obcordate, unequal. $\mathrm{Fl}$. imperfectly separated. H. recepl. U. Fr. slightly ribbed.

54. (Exantur. Cal. large, lanceolate, acute, spreading. l'et. obcordate, radiant, very unequal. $1 \%$ separated. $F$. recept. dilated, depressed. F'r. ribbed, spongy.

55 Cнiтнuия. Cal. small, broad, acute, incurved. Pet. elliptical, acute, incurved, equal. $\%$. united, all perfect. $F$. recept. 0 . Fr. ribbed, coriaceous.

64 Atramanta. Cal. lanceolate, acute, incurved. Pet. obcordate, equal. $F l$. imperfectly separated. $F l$. recept. 0 . Styles short. F'r. ribbed, ovate, hairy.

65 Prmpraria. Cal. O. Pet. obcordate. Fl. either united or diocious. Fl. recept. 0 . Styles capillary, as long as the fruit. Fr. ovate, ribled, witl convex interstices. 
E. Fruit solid, unammed, whoml uings, eompressed laterally, its transverse diumeter being at least twice the hrealle of the juncture.

148 Sium. Cal. small, arute, unequal, or obsolete. J'et. obeordate, or ovovate, equal. Fl. uniform, united. $F$. reeept. 0. Stryles cylind. shorter than the petals. $r \%$ ovate, ribbed, furrowed.

F. Frnit solid, unarmed, compressed transversely, the junchure being broud'r than the transrevse diameter.

151 Airursa. Cal. very small, pointed. L'el. obcordate. Fl. all perfect, slightly radiant. $F l$. reespt. 0. Sieds ovate, convex, with 5 tumid, keeled ribs; interstices deep. angular ; border 0 .

159 Impiratoria. Cal. 0. Pet. olscordate. Fl. all perfect. Fl. recept. 0 . Seeds orbicular, eonvex, notched at each end; with 3 dorsal ribs, and a dilated, flat, even border.

169 Srinum. Cal. small, pointed, spreading. l'et. obcordate, involute: Fl. perfect. Fl. recept. olysolete. Seeds elliptical, convex, with 3 dorsal ribs, and a dilated, Hat. even border.

160 Ancirica. Cal.0. Pet.lanceolate, flattish, unclivided, contraeted at each ent. Fl. perfert. Fl. reept. thim, wayy, narrow, permanent. Seeds elliptieal, convex, with 3 dorsal wings, and a narrow, flat, even border.

161 Ligusticum. Cal. small, pointed, erect. Pet. elliptical, Hattish, undivided, contracted at each end. Fl. perfect. Fl. recept. 0 . Seeds oblong, convex, with 3 dorsal, and 2 marginal, equal wings.

\section{G. Frnit thin, amd almost flut : compressed transrersely, withont} dorsul wings.

170 Peverdanem. Cal. pointed, ascending. I'at. obcordate. 1\% imperfectly separated. F. recept. 0 . Seets elliptical, convex, notched at each end, slightly 3-ribbed; interstices striated ; border narrow, flat, entire.

171 'satisaca. Cul. small, obsolete. l'et. lanceolate, involute. I'. uniform, perfect. Fl. recept. orbicular, wavy, thiu. concealing the ralyx. Seeds elliptical, notched at the summit, with 3 dorsal, and 2 marginal ribs; border narrow, flat, thin, entire.

172 Heracleum. Cal. of 5 small, acule, evalescent teeth. Pet.ob. cordate, radiant. $\%$ separated. $1 \%$ rerept. way. crenate, obture. Sepls obeorclate, notrled at the 
summit; with 3 dorsal rilss, 2 distant marginal ones, and 4 intermediate coloured abrupt lines, from the top; border narrow, entire, slightly tumid.

173 lonbusm. Cal. of 5. awl-shaped, unequal teetl. Pet. obeordate, radiant. Fl. separated. Fl. recent. 0 . Seeds orbieular, roughish, ribless; border tumid, wrinkled or crenate, naked or bristly.

\section{ORDER III. TRIGYNIA.}

* Il. superior.

174 Vhumsu. Cor. 5-cleft. Berry 1-seeded.

175 Sambucus. Cor. 5-eleft. Berry 3-seeded.

* * Fl. inferior.

I176 SrapHylea. Cor. 5-pet. Caps. inflated, 2 or 3 joined together. 1177 ' Trsar. Cor. 5-pet. Caps. 3-valved. Seed feathery.

1178 Corriolola. Cor.5-pet. Seed 1, naked, triangular.

Chenopodium. Stellaria 2.

\section{ORDER IV. TETRAGYNIA.}

179 I'ARAassia. Neet. fringed with bristles terminated by globules. Caps. 4-valved.

\section{ORDER V. PENTAGINIA.}

181 Lixun. Met.5. Caps. 10-celled.

182 sibbalma. Pet. 5. Seeds 5, naked. Cul. in 10 segnents.

180 Sтатісе. P'ct. 5. Seed 1, eovered by the funmel-shaped calyx.

Cerastium 3. Spergula.

\section{ORDVR VI. HEXIGINIA.}

83 Drostus. P'et.5. Caps. 3-valved, many-sected.

\section{ORDER VII. POLYGYNIA.}

.84 Miosults. P'ot. 5, with neetariferous tubular claws. Seed naked.

Cul. of 5 leaves, eaeh spuried at the base. linunculus. 
CLASS V. PENTANDRIA.

ORDER I. MONOGYNA.

LINNEAN NAMES.

ENGLISII NAMES.

MYOSOTIS. SCORPION-GRASS.

1 palustris .... water .........

Wat.pl. Fi.blue 6-8

\begin{tabular}{|c|c|c|}
\hline 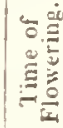 & 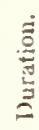 & 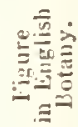 \\
\hline & & \\
\hline
\end{tabular}

2 capitosa .... tufted water ...

Wat. pl. blue 5,6

3 intermedia

trailing hairy

Dry sh.pl. Fi.blue

4,5

B. P?

1973

4 sylvatica

wood

Woods

5 alpestris

r $r$

rock.

6 arvensis

- field.

-

Sc.mou. blue

7,8

P. $* 2559$

7 versicolor.... yellow and blue... San. pl.

blue

$6-8$

1.

2558

LITHOSPERMUNI. GROMIWELL.

1 officiuale ... common ....... Chal hill yellow

4.6

I.

2 arvense...... corn

Corn fi. white

3 purpuro-caru-

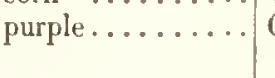

Clial.so.! purple

5,6

(n)

2558

leum

4 inaritimum

Sea sh.

purple

5
7,8

A.

480

ANCIIUSA.

\LKANET.

1 officinalis ....

2 sempervirens..

common

Sea coast purple evergreen ...... Rubbisl ! blue

6,7

$l^{\prime}$.

662

CYNOGLOSSUM. HOUNDS-TONGUE.

1 officinele ... common ....... Rubbish pur. red 6,7

13. 921

2 sylvaticum .. green-leaved .... Sha. la.! blue

13. $16+2$

PULMONARIA. LUNG-WORT.

1 whicinalis ....

rommon ........

Woorls! blue

P. $* 368$

2 angustifolia. 
SPECIFIC CIIAACTlE.

88. MYOSOTIS. Boratrinea. Juss.

1 Cal. funnel-shaped, broad-toothed, rough with close bristles. Clusters leafless : cor. limb longer than the tube : root creeping.

2 Cal. funncl-shaped, broad-toothed: sprinkled with erect bristles. Clusters lcafy at the base: cor. limb the length of the tube: root fibrous.

3 Cal. tube bell-shaped, clothed with hooked bristles; segm. with straight upright hairs. Clusters lcafless : root creeping: stems decumbent.

4 Cal, tube hell-shaped, clothed with looked bristles; segm. with straight upright hairs. Clusters with a leaf at the base: root fibrous : stems ercet.

5 Cal. deeply cleft, clothed with upright hairs, lowermost incurved. Clusters leafless, forked at the base: root fibrous, tufted. $M$. rupicola. Eng. Biot.

6 Cal. cleft half way down, clothed with spreading lrairs; those of the tube hooked. Clusters leafless, with one remote axillary flower : root fibrous.

7 Cal, longer than the partial stalks, hairs of its tube hooked. Clusters on long naked stalks: root fibrous. M. scorpioides. Eng. Bot.

\section{LITIIOSPERUM. Boraginer. Juss.}

1 Seeds smooth: cor. scarccly exceeding the calyx : L. lanceolate, rather acute, veined.

2 Seeds rugose : cor. scarcely cxceeding the calyx : L. obtuse, veinless.

3 sceds smooth : cor. greatly cxceeding the lcnuth of the calyx: L. lanceolate, acute, veinless.

4 Seeds keeled, smooth: 1. ovate, glaucuus, sprinkled with callous points. Pulinonaria. Eng. Bot.

\section{ANCIIUSA. Boraginer. Juss.}

1 Spikes imbricated, 1-rowed, bracteas ovate: I.. lanceolate.

2 Pedunc. axillary, 2-leaved, capitate: L. ovate.

91. CYNOGLOSEUAI. Boraginer. Juss.

I Stam. shorter than the cor: L. broad-lanceolate, downy, sessile.

2 Stam. shorter than the cor: L. spatulato-lanccolate, shining, partly naked, rough beneath.

92. PULMONARIA. Bornginer. Juss.

I Cal. nearly the length of the tube: $\mathrm{L}$. ovate, rough.

2 Cal. nearly the length of the tube: L. all lanceolate, rough. 
C1ASS I. PENIANORA.

OHDER I. MONOGYN1.

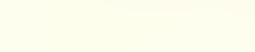

LINNEAN NAMES ENGLISH NMNES.

|

SYMPHITUM. COMFRHI.

1 officinale .... common a a.

2 tubcrosum ... tubr

tuberous-rooted

BORAGE.

1 officinalis

\section{ASPERUGO.}

1 procumbens..

MАDWOR'T.

L YCOPSIS.

1 arvensis

ECHIUM.

1 vulgare...... c

\section{VIPER'S BTGLOSS.}

common .......

I vulgaris ....

BUGLOS心.

2 elatior .......

common ........

3 veris .......

4 farinosa cowsli bird's ey

5 sicotica ..... sicottisl

CYCLAMFN. SOIV-BREAD.

I hederifolium $\oint$.

MEN Y A N'THES.

I trifoliata BUCK-BKAN.

2 mymphieoiles. common fringed Ficla

II oods yellow IV oods! yellow

4,5 4

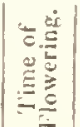

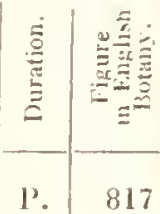

I'.

1502

loi.sh.p. cream

5,6

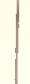


SPECIFIC CIIARACTER.

93. SYMPHITUM. Roragined. Juss.

! L. ovate-lanceolate, decurrent.

2 L. ovate, slightly decurrent ; highest oppositc.

94. BORAGO. Borugines. Juss.

1 C'or. limb nuch longcr than the tube, throat closed by a double row of valves, innemost awl-shaped, bcaring the stamens.

95. ASPERUGO. Boraginer. Juss.

1 Calyx of the fruit Hattened.

96. LYCOPSIS. Boraginea. Juss.

1 L. lanceolate, bristly: cal. crect when flowering.

97. ECHIUM. Boraginer. Iuss.

1 stem tubercled, bristly: L. stem lanceolatc, hispid; spikcs lateral, laairy, deflexed.

98. PRINula. Primulacer. Vext.

1 L. dentatcd, wrinkled : scapc l-flow: limb. of the cor. Hat.

2 L. dentatcd, wrinkled, contracted towards the middle: scapc manyHow: limb. of the cor. Hat.

3 L. dentated, wrinkled, contracted towards the middle: scape manyflow : limb. of the cor. concave.

$4 \mathrm{~L}$. dentated: even, powdery beneath : limb. of the cor, flat: stig. undivided.

5 L. crenate, smooth, powdery: limb. of the cor. Hat: stig. 5-cleft.

99. CYCLAMEN. Primulacer. Vext.

1 Col retroflexed: L. cordatc, angular, denticulate, ncrves and footstalks rough. C. europarum. Eng. Bot.

100. MENYANTHES. Gentiana. Juss.

1 L. ternate: cor. very plumose on the upper surface.

2 L. curdatc, waved, Hoating: cor. ciliated.

101. HOTTONIA. Primulacere. Vent.

1 sitem many-flow: pcluncles in whorls. 
LINNEAN NAMES.

LYSIMACHIA. LOOSESTRIFE.

1 vulgaris yellow
tufted

Wat. sh. yellow

2 thyrsiflora....

Bog. pl.! yellow

7

7

P.

761 **

3 nemorum ....

4 Nummularia.

wood ........... Moi.sh.p. yellow

moncywort

Bog.pas. yellow

$5-9$

$P$.

527

ANAGALLIS.

1 arvensis

\section{PIIPERNEL.}

2 cxrulea ....

scarlet..........

3 tenella ......

Fi.Ro.si. scarlet

Corn fi.! blue

6,7

A.

529

blue

Boggy 1

pl. ruse

6,7 A.

1823

104

AZALFA.

AZALEA.

1 procumbens .. trailing ........ Sc. moun. ruse

105

CONVOLVULUS

IBINI WEED.

1 ar'vensi

...

small

Fi.Ro.si. pink

6,8

P.

312

2 sepium...

great

Moi.hed. whitc

7,8

P.

313

3 Soldanella Sea sh. flesh

POLEMONIUM. IACOB'S-LADDFR.

1 caruleum .... b bluc

$$
\text { ......... Bushy p.! blue }
$$

CAMPANLTA. BELL-FLOWIR

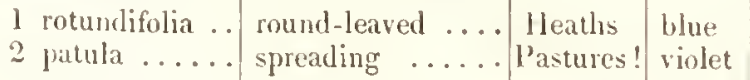

8,9

$P$.

866

7,8

b.

3 Rapunculus.. rampion ....... Hedge b. purple 7,8

l'.

283

4 persicifolia .. peach-leaved ....

Sc. wds. white

P.

5 latifolia...... griant 


\section{SPFCIFIC CIIARACTER.}

102. LISIMACIIA. Primulacer. VENT.

* Peduncles many-flow.

1 Panicled, racemes term: 1. ovate-lanceolate, acuie.

2 Racemes lateral, axillary, peduncled.

$$
\text { * Peduncles 1-flow. }
$$

3 L. ovate, acute: Fl. solitary : stcm procumb: stam. smooth. 4 L. somewhat cordate: Fl. solitary: stem creeping: stam. glandular.

\section{ANAGALLIs. Primulacer. Vent.}

1 L. ovate, dotted beneatl : stem procumbent : cor. minutely notched.

$2 \mathrm{~L}$. lanceolate-ovate, dotted beneath : stem erect: cor. strongly crenate.

3 L. roundish, rather acute, petioled: stem creeping: stigma acute.

\section{A\%ALEA. Ericer. Juss.}

I Branches diffuse, procumbent: L. oppositc, revolute, very smooth.

\section{CONVOLVULUS. Convolvulacer. Juss.}

1 L. arrow-shapcd, lobes acute: pedunc. mostly 1-flow: bracteas minute, remote from the flower.

2 L. arrow-shaped, lobes iruncated : pedunc. square, 1-flow : bracteas cordate, close to the calyx.

3 L. kidney-shaped, pedunc. 1-flow. with membranous angles: stems procumbent.

\section{POLEMIONIUM. Polemoniacer. Juss.}

1 L. pinnated : Fl. erect : cal. longer than the tube of the corolla: root fibrous.

\section{CAMPANULA. Campamelarea. Juss.}

1 L. radical kidney-shaped, serrated; those of the stem lincar, entire.

2 L. straight; radical lanceolate-oval : pan. spreading : cal. denticulated. Stem witl several fringed angles.

3 L. waverl; radical lanceolate-oval: pan. compact. Cal. entire : stem angular, hairy below.

4 L. smooth, slightly serrated; radical obovate; stem linear-lanccolate, sessile, remote. Stcm round, smooth, few-flow.

5 1. ovate-lanceolate: stem very simple, cylind : pedunc. 1-flow : fruit drooping. 
ORJER I. MONOGYNA.

$=$

LINNEAN xitus.

CAMPANULA.

6 rapunculoides.

ENGLIGI NAMES.

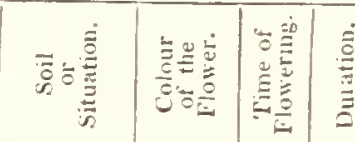

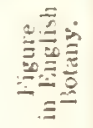

7 Trachelium ...

creeping..$\cdot$.

Woods! blue

$\begin{array}{ll}7,8 & 1\end{array}$

1369

8 glomerata....

nettle-leaved ....

Woods violet 7 l'.

12

8 gromerata...

chustered........

Chal. pa. violet

7,8

I'.

90

9 liybrida ....

10 hederacea....

corn

C

ivy-leaved ........

Chal. fi. violet 8 A

375

Moi.sh.p! blue 6-8 P.

73

PIIYTEUMA.

RAMPION.

1 orbiculare....

round-headed .... Chal. pa.. violet

J ISIONE.

SHEEP'S-SCABIOUS.

J montana com

J.OBEI.IA.

1 Dortmanna...

\section{LOB FLIA.}

2 urens

ivater

acrid

\section{IIPATIENS.}

1 Nolimetangere

IIOLA.

l liirta.

2 otorata.

3 palustris ....

4 canina

5 lactea

6 Hiticornis.

7 tricolor

\section{BALSAM.}

yellow.

VIOLET.

- hairy

sweet

marsl ..........

. 1

$\log$.

crean-coloned y

yellow-spurred

pans
- Lakes.. blue

Heaths! blue

$$
\text { S }
$$

San. pas. blue

6,7

A.

$88 \cdot 2$

$7 \quad \mathrm{I}^{3}$

140

$8,9 \cup \mathrm{P}$.

953

937

\begin{tabular}{ll|l|l} 
W.sh.pl! yellow & 7,8 & 1.
\end{tabular}

\section{7}

Chal. so. blue 4 P'.

894

Sha. pl. purple 3,4 l'.

619

Mlos.log hlue 4 I'.

44

lleaths blue 4-8 I'.

$6: 20$

M. liea. creim 5 l'.

445

Pa

Cult.grr. yel. pu. 5-9
I'.

1. 


\section{CAMTANLA.}

6 L. cordate-lanceolate: stem branched: Fl. scattered, l-lowed, nodding: cal. refiexed.

7 Stem angular: L. cordate-lanceolate, sharply serrated: cal. hispirl: pedunc. axillary, few-flow.

8 Stem angular, simple: Fl. srssile, in a trminal head: L. ovate, crenate.

9 Stem often branched at the base, straight; J. oblong, crenate, undulated : cor, shorter than the calyx: caps. prismatic.

10 L. cordate, 5-lobed, petioled, smootl : sten lax.

\section{PHYTEUMA. Campanulacea. IIIss.}

l Ilead roundish: I. crenate; radieal ones cordate.

109. JASIONE. Lobeliaced. JUSS.

1 L. oblong, bluntish, wavy, sessile, alternate.

\section{T,OBFLIA. Lobeliaced. Itiss.}

1 L. linear 2-celled, very entire: stem nearly naked.

2 Stem rather upright : L. lower olsovate, denticulated; uppermost lanccolate, serrated : Clusters terminal.

111. IMHPTIENS. Balsumines. Ricn.

1 Pedunc. many-flow. solitary : L. ovate: joints of the stems swelling.

112. VIOLA. Tiolacea. Juss.

l Stemles: : L. cordate, rough with lıairs, as well as their petioles : cal. obtuse: lateral pet. with a hairy central line.

2 Stemless: scyons creeping: $L$. cordate, smonthish, as well as thrit petioles: cal. obtuse: lateral pet. with a hairy central hine.

3 Stemless: 1. kidney-shaped, smooth : root creeping: lateral pet. with a liairy central line.

4 Sten when becoming adult, aseending, ehannelled: I. oblong-cordate : cal, acute: stip. serrated: bract. awl-slaped, entre.

5 Stem ascending, cylind: L. ovate-lanceolate: stıp. inciso-sermated: bracl. lanreolate, somewhat serrated.

6 Stem ascending, wondy, angular, branched: L. eordate, eoriaceous, smooth: Cal. lraves laneeolate: stip. and braet. fringed.

7 Stem angular, diffuse: 1. ololong, deeply erenate: stip. lyrate, piuuatifir]: bract. obsolete. 
VERBASCUM.

1 Thapsus ....

2 Lychnitis.... wh

MULLEIN.

8 lutea....... yellow mountain.

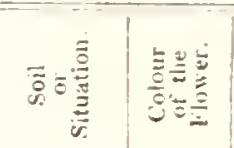

LINNEAN NAMES.

MISII NAMES.

VIOLET:

3 pulverulentum

great ......... Chal. so. yellow

white ........ Chal. so. cream

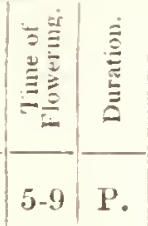

721

4 nigrum......

yellow hoary .... I

black ......... Chal.so. yellow

7,8

B.

549

5 virgatum ....

large-flowered....

6 Blatiaria ...

moth

Grav.pl.! yellow

7,8

P.

114

115

H YOSCYAMUS.

DATURA.

THORN-APPLE

I stramonium

common ........

Riubbis 1 white

7 A.

$1: 288$

1 niger.......

HINBANE。

1 niger. common

l Belladonna .. common ....... $\mid$ Rublish $\mid$ violet

\section{SOLANUII.}

NIGHTSIIADE.

1 Dulcamara ..

2 nigrum.....

woody.........

common ....... Rubbish white 6-9 A.P.

565

566

\section{ERYTHRAA. CENTAURY.}

1 Centaurium .. common ........ Grav. pa. rose $\quad 7,8 \mid$ A.

2 littoralis .... dwarf-tufted .... Sea coa. rose 
SPECIFIC CIARACTER.

VIOLA.

8 Stem triangular, simple: 1. ovate, crenate, ciliated: stip. Iobed palmate: bract. minute, scarcely toothed: spur, the length of the calyx.

\section{V'ER13\SCUM. Solaner. Juss.}

1 L. decurrent, woolly on both sides: stem simple.

2 L. oblong-wedge-shaped, almost naked on the upper surface: stem angular, panicled.

3 L. ovate-oblong, slightly serrated, powdery on both sides: stem. crliud. panicled.

4 L. oblong-cordate, petioled, crenate, undulated, somerimes pubescent.

5 L. oblong-lanceolate, dentate, sessile: radical ones somewhat lyrate, pubescent: stem branched: Fl. clustered, almost sessile.

6 L. embracing the stem, oblong, smooth, serrated, radical sinuated: pedunc. 1 -flow. solitary : pedunc. longer than the bracteas.

\section{DATURA. Solaner. Juss.}

1 Pericarps spinous, erect, ovate: L. ovare, smooth, sinuated.

115. IIYOSCYAIIUS. Solanere. .luss.

I L. embracing the stem, sinuated: Fl. sessile.

116. ATROPA. Solanea. Jiss.

1 Stem herbaceous: L. ovate, entire: F'l. solitary.

117. SOLANUMI. Solanea. Juss.

1 Stem unarmed, shrubby, flexuose: upper leaves hastate: racemes cymose.

2 Stem unarmed, herbaceous: L. ovate, dentate, waved: umbels lateral, nodding.

\section{ERYTHRAA. Gentianc. Juss.}

1 Stem dichotomously panicled: L. ovate-lanceolate: cal. shorter than the tube; seg. partly combined by a membrane.

2 Stem simple, straight: L. linear-obovate, obscurely 3-ribbed : cal. as long as the tube; teeth awl-shaped: Fl. corymbose, nearly sessilp. 


\section{CLASS T. PENTANTIRIA.}

URDER 1. MONOGYNHA.

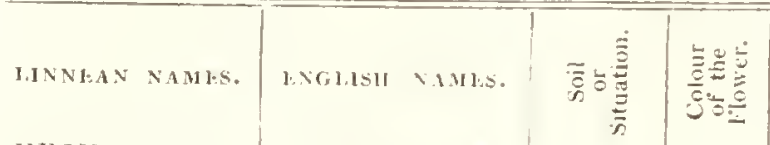

ERTIIR ASA.

3 latifolia...... broad-leaved .... Sea sh. rose

4 pulchella .... dwarf-branched .. sea coa. rose

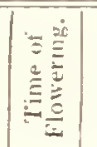

\begin{tabular}{|c|c|}
\hline 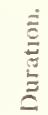 & 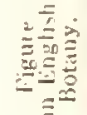 \\
\hline & \\
\hline
\end{tabular}

SAMOLUS.

\section{BROUKWEED.}

I Vralerandi... commols.......W W. grav. whitc

LONICHRA. HONEYSIC'KLE.

1 Caprifolium ...

2 Periclymenum

3 Xylosteum .. pale perfoliate. .

Woods! ye!low 7

$\lambda$

$\int 8,9 d$

+...

\section{RHAMNUS.}

common

Iledges yellow $6-10$

upright

\section{IVoods! yellow}

1 catharticu

BTCKT1IORN.

2 Frangula ....

purging .......

Hedges

\begin{tabular}{l|c}
7 \\
\end{tabular}

Meds

alder-leaved

. 11 oods

grcen

is.

1629

800

S. 916

EUONYUUS. SINDLE-TREE

I curopaus....

common ........ llenges

white

$36^{\circ} 2$

$$
\text { RIBHS. CURRANT. }
$$

1 rubrum......

common ........ Riv ban. yell-ish

"2 petraeum ....

$r$

3 spicatum ....

4 alpinum ....

acid mountain.... Nor.wds ! green tast

5 nirrum $\ldots . .$. blark ess mont untain Nor.wds! green (1)

S. .250

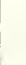

6 Grosiularia .. rough gooseduerry . Hedges lor.t the.

$\therefore$ I. 1289

s. 705

s. $\quad 1290$

S. 704

S. $\quad 1: 291$

S. $\quad 1 \div 92$ 


\section{ERITIIRAL.}

3 Sitem 3-cleft at the top. Cal. as long as the tube. Fl. in dense forked tufts: L. broadly elliptical, 5 or 7 -ribbed.

4 Stem winged, much branclied on all sides: L. ovate: cal. segments awl-shaped, shorter than the tube: Fl. solitary, stalked.

\section{SAMOLUS. Primulacer. VENT. \$}

1 L. obtuse: raceme many-flow: pedicels furnished with bracteas.

\section{LONICERA. Caprifoliacer. Juss.}

l Fl. ringent, whorled, term: L. deciduous; upper united and perfoliate.

2 Heads ovate, imbricated, term: L. all distinct, deciduous : Fl, ringent. 3 stalks 2 -flow: berries distinct L. entire, downy.

\section{RHAMNUS. Rhamnea. Juss.}

1 Spines term: Fl. 4-cleft, diøcious: L. ovate, serrated: stem erect: berry 4 -seerled. Style 4 -cleft.

2 Unarmed: Fl. hermaph. L. very entire, smooth: berry 2 -seeded. Style simple.

\section{EUONYMUS. Celustrimers. R.Br.}

1 Fl. mostly 4-cleft: pet. acute: branches smooth.

123. RIBES. Grossulacer. Juss.

* Unarmed. Currants.

1 Racemes smooth, pendulous: Fl. flattish: pet. obcordate.

2 Racemes hairy, erect: fruit pendulous: pet. obtuse, bract. shorter than the flower-stalks: Stem erect.

3 Spikes erect: Fl. nearly sessile : pet. oblong; bract. shorter than the flower-stalks.

4 Racemes erect: bract. longer than the flower-stalks: I. shining beneath: stem erect.

5 Racemes, hairy, pendulous, with a separate flower-stalk at the base of each, Fil. oblong.

$$
\text { ** Prickly. Gooseherries. }
$$

6 Prickles 1-3. under each bud: branches otherwise smooth, spreading: pedunc. 1-flow: bract. separate: fruit hirsute: cal. seg. reflexed, shorter than the tube. 
CLASS V. PENTANDRIA.

ORDER I. MONOGYNIA.

$=1$

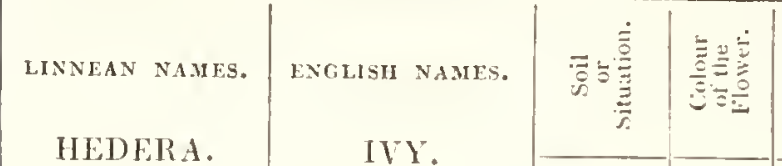

1 Itelix eommon

IILECEBRUM. KNOT-WEED.

1 verticillatum.

whorled

GiLAX.

1 maritima .... THESIUM.

1 linophyllum .. flax-leaved ...... Chal. pa. white

\section{VINCA.}

1 minor ......

2 major

\section{PERIWINKLE.}

$$
\text { less }
$$

greater
IVY.

Wds.lla green

10

S. 1267

SILT-WEED. black ......... Salt mar. flesh BASTIRD-TOAD-FLAX.

\section{Bushy pl! violet Wet grov. blue}

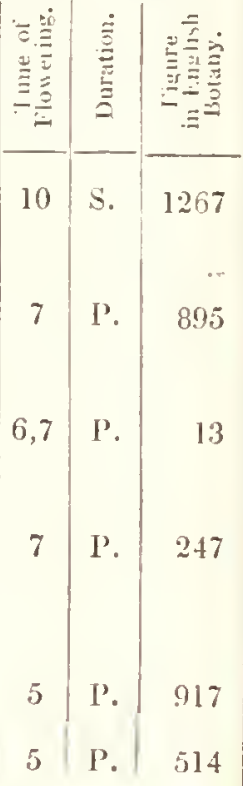

ORJER II. DIGINIA.

129 IIERNIARLA. RUPTURE-WORT.

1 glabra ..... smooth ....... San. gr. green

2 hirsuta..... hairy ........ San. gr.! green

\begin{tabular}{|c|c|c|}
7,8 & P. & 206 \\
7,8 & I. & 1379 \\
& & \\
& & \\
5,6 & P. & 1033 \\
8,9 & A. & 717 \\
8 & A. & 1721 \\
8,9 & 1. & 2247 \\
8,9 & A. & 1722 \\
\hline
\end{tabular}


124. HEDERA. Caprifoliacer. Juss.

1 L. some ovate, some lobed.

125. ILLLCEBRUMI. Illecebrer. R.Br.

$1 \mathrm{Fl}$. whorled, without braeteas: stems procumbent.

126. GLAUX. Primulacer. VENT.

1 Stem erect, leafy: FI. solitary, axillary.

127. TIIESIUM, Santalacer. R. BR.

1 Spike brancherl: braeteas ternate: L. linear-lanceolate: tube of the calyx very short.

128. VINCA. Apocunea. Juss.

1 Stems proeumb: L. elliptic-laneeolate, margin smooth : Fl, peduncled: ealyx segments lanceolate.

2 Stems uprightish: L. ovate, ciliate: Fl. peduncled; calyx segments setaeeous, elongatcd.

\section{ORDER II. DIGYNIA.}

129. HERNIARIA. Hleccbrea. R. BR.

1 Herbaceous: leaves and calyx snooth.

2 Herbneeous: leaves and calyx hairy.

130. CHILYOPODJUM. Chenopoder. VENT.

* Leates angular.

1 L. triangular, arrow-shaped, entire: spikes term. eompound, leafless.

2 L. triangular, denticulated: spikes erowderl, very straight, approximate to the stem, very long; almost leafless.

3 L. rhomboid-triangular, sinuate-dentate: spikes crect, eompound, leafy.

4 L. triangular, somewhat toothed, upper ones bluntish : spikes erect, eompound, rounded, leafy.

5 L. ovate, shining, acute, dentate: panicles much branehed, cymose, leafless. 


\section{CLASS Y. PENTANDRIA.}

ORDER II. DIGYNIA.

LINNEAN NAMS. | ENGLISH NAMES.

6 hybridum .... maple-leaved ....

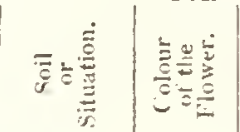

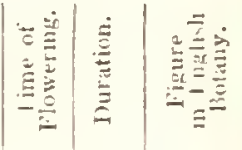

7 album ..... white .........

Rubbish

green

8

A.

1919

8 ficifolium.... fig-leaved ......

Rubbish

green

7,8

1.

1723

9 glaucum .... oak-leaved ......

Dung hill green

8 A.

$17: 4$

Rubbish green

8

A. 1454 * 步

10 olidum ..... stinking ....... Rubbish

11 polyspermum . round-leaved .... Rubbish

12 acutifolium .. sharp-leaved .... Uncul.gr.

13 maritimum .. sea ......... Salt mar.

green

green

green

7,8

A.

1034

A.

1480

7,8

A.

1481

BET $A$.

BEF:T.

1 maritima

\section{SALSOLA.}

1

Kiali.

$\ldots$

2 fruticosa ....

\section{SALTIVORT.}

Sea coast green

$7,8 \cdot 1$.

633

green

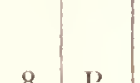

285

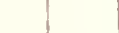

(1)

7 A.

634

Sea shore Alesh

Sea shore green

\begin{tabular}{|c|c|c|c|c|c|c|}
\hline ULN1US. & ELM. & & & & & \\
\hline 1 campestris .. & common ........ & IJedges & brown & 3,4 & T. & 1886 \\
\hline 2 suberosa ... & cork-barked & Iledges & reddish & 3 & T. & 2161 \\
\hline 3 majors...... & dutch ............ & Jledges & brown & 3 & $\mathrm{~T}$. & 25.42 \\
\hline 4 montana ... & broad-leaved .... & Hoods & brown & 3,4 & T. & 1887 \\
\hline 5 glabra ..... & wych $\ldots \ldots \ldots$ & Hedges & brown & 3 & $\mathrm{l}$. & 2248 \\
\hline
\end{tabular}




\section{SIECIFIC CIIARACTER.}

\section{CIIENOPODIUM.}

6 L. cordate, angular-dentatc, acuminatc: panicles much branched, subeymose, divaricate, leafless.

7 L. rlomboid-ovate, jagrged, entire towards the base, uppermost ollong, entire: seeds smooth.

8 L. hastate-sinuated, jagged, entire towards the base; uppermost oblong, entire: serils dotted.

9 L. all oblong with deeply waved edges, glaucous beneath: spikes leafless, clustered. šeeds minutely dotted.

$$
\text { ** Leaves entire. }
$$

10 L. rhomboid-orate: spikes conglomerate, leafless.

$: 11$ L. ovatc : stem prostratc : clusters cymosc, divaricate, leafless.

:12 L. ovate, acute: stem erect: clusters spiked, compound, erest, elongated, leafl : lowermost, in strong plants, cymose, leafless.

13 L. awl-shaped semicylind: Fl, axillary, sessile.

\section{BE"'L. Chenopolea. Yext.}

1 Stems decumb. Fl. in pairs : calyx-segments entire at the keel.

\section{SALSOLA. Chenopoder. VExt.}

1 Herbaccous, decumbent: L. awl-shaped, spinous-pointed, rough: cal. marginate.

2 Shrubby erect: I. semicylind: rather obtusc, spineless.

\section{U1.MUS. Ulmacer. Mirb.}

1 L. cloubly-serrated, scabrous, unequal at the base: Fl. subsessile, 4-cleft : fruit oblong, deeply cloven, naked.

2 L. pointed, doubly serrated, rough, unequal at the base: Fl. peduncled 4 or 5-cleft: fruit round, decply cloveı, naked: branches spreading: bark corky.

$3 \mathrm{~L}$. unequally-serrated, rough, unequal at the base: Fl. subsessile, 4-cleft: fruit obovate, slightly cloven, naked; branches drooping : bark corky.

4 L. doubly-scrated, acuminate, round, uncqual at the base: Fl. peduncled, 5 or 6-eleft: fruit round, slightly cloven, naked: branclies drooping.

5 L. doulbly serrated, smooth, unequal at the base: Fl. subsessile, 5-cleft : fruit oblong, naked, deeply cloven. 


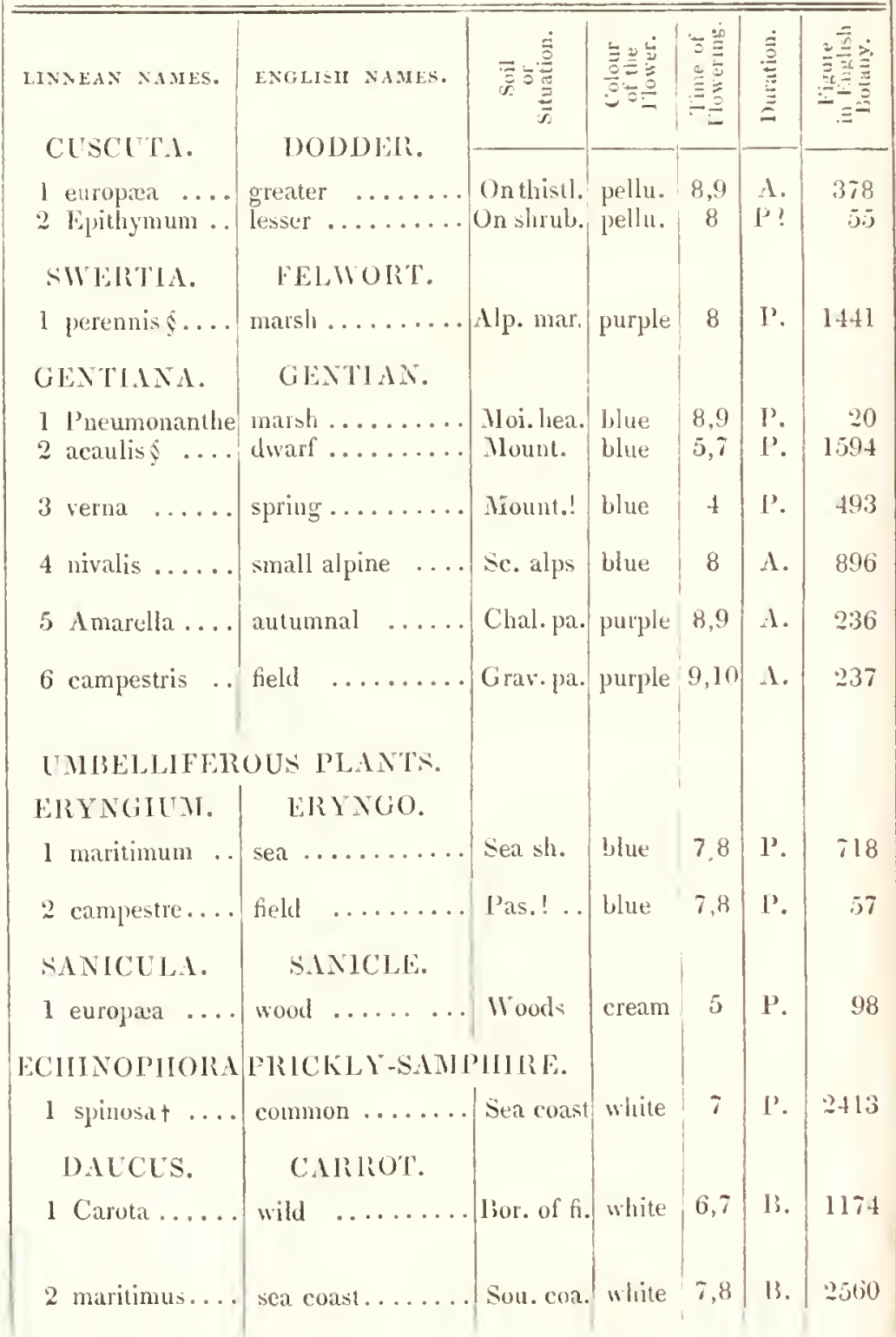


SPICIFIC CIIIRACTIR.

134. CUSCLTA. Convolvulacer? Juss.

1 Fl. nearly sessile: cor. throat naked : stig. acute.

2 Fl. sessile: stam. with minute crenate scales at the base: stig. acutc.

\section{SWER'TIA. Gentiance. Juss.}

1 Cor. 5-cleft: radical lcaves ovate.

\section{GENTINAA. Gentiand. Juss.}

1 Cor. bell-shaped, 5-cleft: Fl, peduncled : L. lincar.

2 Cor. 5-cleft, bell-shaped solitary : stem 4-angular, scarcely the length of the flower.

3 Cor. 5-cleft, salver-shaped, crenate : scoments toothed at the base: $L$. ovate, crowded.

4 Cor. funnel-shaped, 5-cleft, with notched intermediate scgments: cal. angles equal, acute: stem many-flow.

5 Cor. 5-cleft salver-shaperl; throat bcarded: cal. segments nearly equal.

6 Cor. 4-cleft salver-shaped; throat bearded: outer calyx-segments larger.

UMBELLIFEROUS PLANTS. Umbelliferc. Juss. 137. ERYNGIUM.

1 L. radical roundish, plaited, spinous: heads peduncled: Recept. scales 3-cleft.

2 L. embracing the stem, pinnatifid. Recept. scales undivided.

138. SANICULA.

1 L. radical simple, deeply lobed. Fl. ncarly sessilc.

\section{ECIINOPIIORA.}

1 Lcaflets awl-shaped, spinous pointed, entire.

\section{DAUCUS.}

1 L. pinnatifid, with lincar-lanceolate segments: umbels with one central, ncutral, blood-red flower;--when in seed concave. Seedbristics slender.

2 L. pinnatifil, with rounded scgments, succulent. Umbels when in seed convex. Secd-bristlcs flattencd. 
CLASS I. PENTANDRIA.

ORDER H. DIGYNIA.

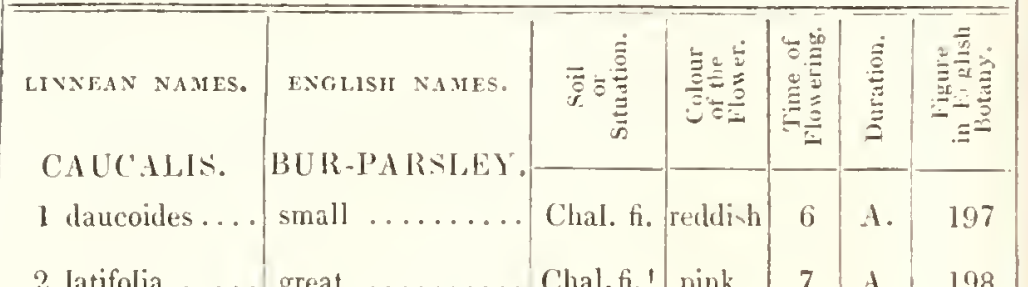

2 latifolia Chal.fi.! pink

TORISIS. IIEICE-I'ARSLEY.

\begin{tabular}{ll|l|l|l|l|l}
1 & Anthriscus .. upright $\ldots . .$. & Herges & white & $\tau$ & A. & $* 987$
\end{tabular}

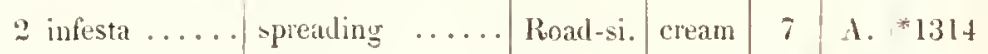

3 nodosa ..... knotted .......

ANTHRISCUS. BEAKED-PARSLEY.

\begin{tabular}{ll|l|l|l} 
l vulgaris $\ldots .$. & common ....... Waste gr. \\
SCANDIX. & SHEPHERD'S-NEEDLE.
\end{tabular}

145 CHAROPHYLUM. CIERTIL.

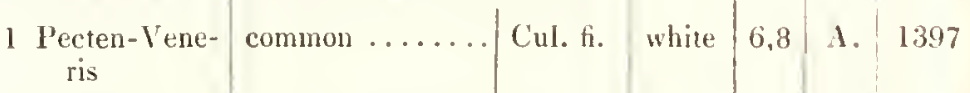

I sativum $\oint \ldots$. garden......... IVa

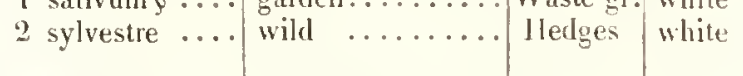

Waste gr.' white

6 1.....

\begin{tabular}{l|l}
4,5 & P. \\
& 752
\end{tabular}

\section{MYRRHIS.}

1 odorata.

2 temulenta...

3 aurea

4 aromatica....

BUNIUN.

1 flexuosum....

\section{CICELY:

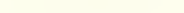

rough ............

tawny-seeded ....

....

broal-leaved .... scotland! white EAR'TI-NUT.

common ....... Pant. . white 


\section{CAUCALIS.}

1 Umbels trifil, nakcd: umbeltets 3-seeded : partial bract. 3: L. morc than doubly compound.

2 Umbels trifid, with membranous bracteas: umbellets 5-seeded: L. pinrate, serrated.

\section{TORILIS.}

1 Umbels many-cleft, crowded: bract. severaI. Leaf. pinnatifid: branches nearly upright. Caucalis. Eng. Bot.

2 U'mbels many-cleft, crowded: gcneral bract. few or none: partial many. Leafl. pinnatifid : branches spreading. Caucalis. Eng. Bot . 3 Umbels lateral, simple, nearly sessile: stem prostrate. Fr. partly granulated. Caucalis. Eng. Bot.

\section{ANTHRISCUS.}

1 L. triply pinnate, pinnatifid. Fr. ovate, twice the length of its beak. Scundix. Fng. Bot.

\section{SCANDIX.}

1 Umbels simple, solitary, or in pairs : bract. jagged: Pet. inflexed at the point. Fr. witls a long bristly-edged beak.

\section{CIIROPHYLLUN.}

1 Umbels sessile, Iateral: bracteas lanccolate.

2 Umbels term. pedunc: bracteas ovate, membranous.

\section{MYRRHIS.}

1 Seeds with sharp prominent angles; roughish towards the summit. Scaudix. Eng. Bot.

2 Seeds furrowed, nearly smooth. Stem rough, swelled under each joint. Charophyllum. Fing. Rot.

3 Seeds ribbcd, col : stem slightly swelled, angular, hairy: L. pointed, pinnatifid: general bracteas few; partial deflexed. Charophyllum. Jing. Bot.

4 Seeds ribbed. Styles elongated. Leaflets ovate, acute, serratcd undivided.

\section{BUNIUM.}

1 stcm tapering and zig-zag at the basc: styles nearly upright. lir. beaked : gencral bracteas $1-3$. 
CLASS V. PENTANDRIA.

ORDER II. DIGYNIA.

LINNEAN NAMIES. ENGLISII NABLS.

SHUN. WATER-PARSNEP.

I latifolium .... broad-leaved ....

के⿻三丨口

Rivulets

white

它䓠

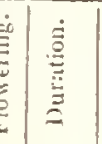

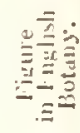

149

150

151

152

153

2 angustifolium. narrow-leaved ...

Rivulets

3 nodiflorum .. procumbeit ....

Rivulets white $7,8 \mathrm{P}$.

639

4 repens ..... crecping ....... Bog.mea. white $6-8$ P'.

1431

5 inundatum .. least

Ditches white

6 verticillatum

salt mar. white SISON.

IONE-WORT.

1 Amoinum...

hedge ..

.

2 segetum eorn

Chal. fi.! white

5

$I^{\prime} ? 227$

CORIANDRUMI. CORIANIDER.

1 sativum $\$ \ldots$ common ....... Fields

(ENANTIE. WATER-DROP-WORT.

1 fistulosia .... common ....... Ditches

2 pimpinelloiles

parsley

Salt mar. white

3 peucerlanifolia

Ditches white

204

139

227

*395

951

228

8 A.B?

$-28$

COWBANE.

1 virosa ..... water ........ Ditches white

$\mathrm{P}$.

479

white $7,8 \quad 1$.

1192

」リMLOCK.

IIedges

white

6,7

b.

$119 \mathrm{~J}$

white

6

A.

67

7,8 P. 363

7 P. 347

6 Y. 348 


\section{SPECIFIC CHAIACTER.}

\section{SIUM.}

1 L. pinnate: leat. oblong lanceolate, equally serrated.

2 L. pinnate: leafl. unequally lobed and serrated: umbels peduncled, opposite the leaves: stem erect.

3 L. pinnate: leafl, orate, cqually serrated: umbels nearly sessile, opposite the leaves: stem procumbent.

4 L. pimate: leafl. roundi.h, deeply toothed: umbles pedunclcd, opposite the leaves: stcm creeping.

5 L. pimnate, cut; lowermost in many compound, capillary segments. tmbek 5-How. in pairs. Sison. Eng. Bot.

6 Leafl. whorled, capillary, many-cleft. Sison. Eng. Bot.

\section{SISON.}

I L. pinnate; uppermost ternate: umbels erect, about 4-rays: bases of the styles globose.

2 L. pinnate: leafl. roundish, numerous: umbels drooping variously: bases of the styles dilated, depressed.

\section{CICLT $\lambda$.}

1 L. bi-ternate: Leaf. lincar-lanceolate, decurrent.

\section{ATHUSA.}

1 L. uniform: leafl. wedgc-shaped, decurrent, with lanceulate seg.

\section{CONTUM.}

1 Stem polished and spotted, much branched.

\section{CORIANDRUM.}

1 The two hemispherical seets making onc uniform globe: leafl. of the lower leaves wedge-shaped.

\section{GN ANTIIE.}

1 Stoloniferous : stem leaves pinnate, filiform, fistulous: gencral bracteas mostly wanting.

2 Rardical leafl. wedge-shaped, cloven; those on the stem linear, very entire, elongated : general bracteas, many, linear.

3 Leaf. all linear: general bracteas none: knobs of the root sessile, clliptical. 
ORDER II. DIGYNIA.

LIVFAV LINNEAN NAMES.

ENGLISII NAMES.

\begin{tabular}{|c|c|c|c|c|}
\hline$\overline{\mathscr{D}}$ & $\begin{array}{l}50 \\
5=0 \\
0\end{array}$ & 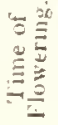 & $\stackrel{\bar{\Xi}}{\stackrel{\Xi}{E}}$ & 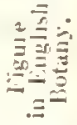 \\
\hline RT & & & & \\
\hline
\end{tabular}

QENANTIE.

WATER-DROP-WORT.

4 crocata......

hemlock ........ I Jitches

5 phellandrium.

fine-leaved ...... Rivulets

white

7 P. 2313

CRITHMUM.

1 maritimum ..

S. 11 IIIIIRE.

SMYRNIUMI.

1 Olusatrum ... common ........ APIUM.

1 graveolens ... smallage......

EGOPODIUM. GOAT-WEED.

1 Podagria .... common .......

IMPERATORIA MASTERHORT.

1 Ostruthium...

ANGELICA.

1 Archangelica $\$$

2 sylvestris ....

LIGLSTICUM.

1 scoticum ....

2 Cornubiense. . great .......... Scot.men. flesh

Ditches gre-ish 8,9

Rocks.

gre-ish 5

H.

230

819

\section{\NGELIC 1 .}

garden ........ Wat. pl.! green wild ......... Wat.pl. Hesh

P.

1128

Scottish ....... Nor. coa, reddish

LOVAGI:

P. 1207

I'. 683

MEU.M.

SPIGIEI.

1 athamanticum. bald-money .... Nor.pas.yell-ish 5,6

I. 2249

2 Funiculnu .. conmon fennel .. Cha. chif. goldco.

\section{CARLil.}

C $A R \perp W N Y$

I ('anif..... conmon ....... Mea.pas. white 
SPECIFIC CHARACTER.

\section{OENANTHE.}

4 Leafl. all wedge-shaped, many-cleft, nearly equal.

5 Segments of the leaves divaricated. Fr. ovate, with 5 broad ribs. Phellandrium. Eng. Bot.

\section{CRITHNIUM.}

1 Leafl. lanceolate, flcsliy: bracteas ovate.

\section{SMIYRNIUM.}

1 stem-leaves ternate, petioled, serrated.

\section{AI'IUN1.}

1 Leafl. of the stem-leaves wedge-shaped: stem furrowed: partial bracteas 0 .

$$
\text { 158. EGOPODIUN. }
$$

1 L. radical bi-ternate; uppcrmost, ternate, ovate.

\section{IMPERATORIA.}

1 L. bi-ternate, undivided or 3-lobed, rough-edged: pedunc. alternate.

$$
\text { I60. ANGELICA. }
$$

1 Terminal leaflet lobed.

2 Leafets equal, ovate, scrrated.

\section{LIGUSTICUM.}

1 L. bi-ternate.

2 L. radical decompound, cut; those on the stem ternate, lanceolate, entire: furrows of the seed indistinct.

\section{MEUM.}

1 Leafl. in many sctaceous scgments: bracteas both general and partial.

2 L. tri-pinnate: leaf. awl-shaped, drooping: bracteas 0.

\section{CARUMl.}

1 Sitem branched: partial bracteas 0 . 
CLASS V. PENTANDRIA.

ORDER II. DIGYNIA.

\section{ATIAMANTA.STONE-PARSLE}

1 Libanotis.... mountain ..... Cha.hills! white

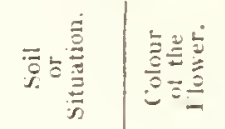

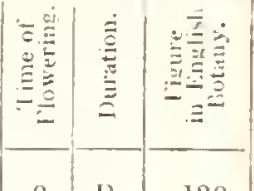

PIMPINELLA. BURNET-SAXIFRAGE.

I saxifraga .... common

. Chal pas. white

7,8

$2 \operatorname{magna} . . .$.

Chal.so. white

7,8

P.

138

3 dioica

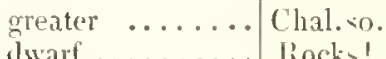

cream

\section{CNIDIUM.}

PEPI'ER-SAXIFRAGE.

1 Silaus ..... Neadow ........ Moi. men. yell-ish 8,9 P'

2142

\section{BUPLELRUH. HARE'S-EAR.}

I rotundifolium. common ....... Chra. fi. yellow

2 Odonites.... narrow-leaved.... Rocks.. cream

3 tenuissimum... slender Salt mar. yell-ish 8,9

IIDROCOTYLE. WHITE-ROT.
1 vulgaris No. hea. white

5,6

P.

751

SLLINUM.

NILK PARSLEY

I palustre marsll

Marshes white

7 13.P?

229

PEUCEDANUM.SULPIIRUOR'T

1 officinale .... sea ........... Sial. mar.! buff .

$7-9$

l'.

1767

\section{PASTINACA.}

\section{PARSNEP.}

1 sativa ..... common ....... Bor. of ti. yellow

HLRACLEUN. COW-PARSNEP

1 Spondylium .. common ........ Bur. of fi. white

TORITLIUM.

HARTWORT.

I officinale $\$$ small ......... Cult. gr.! white 


\section{ATHAMANTA.}

1 L. bi-pinnate, cut: umbels henispherical.

\section{PIMINIELLA.}

1 L. pinnate: leatl. of the radical ones roundish, of the uppermost in livear segments.

2 L. pinnate: leafl. ovate, serraterl, somewhat cut; terminal one 3-lobed.

3 I.catl. all nearly linear: umbels panicled: Fl. dicecious.

\section{CNIDIUMI.}

1 LeaA. dceply pinnatifid; their segments oppositc, decurrent. General bracteas 1 or 2 .

\section{BUPLEURUM.}

1 Gcneral liracteas wanting: L. perfoliate.

2 Partial loracteas 5: acute, 3-ribbed; general 3-4: branches widely spreading: umbels stalked. L. lanceolate.

3 Umbels simple, alternatc, 3 -flowered, with 5 . aw]-shaped bracteas.

\section{IIYDROCOTYLF.}

1 L. orbicular, peltate, smooth, cloven at the base: umbels aggregate: Fl. ncarly sessile. 169. SELINUM.

1 Milky: I. tri-pinnate: leaf. pinnatifid: rays of the umbel rough: ribs of the seeds broad and olstuse.

\section{PEUCEDANUM.}

1 L. 5 times 3-cleft: leaf. lincar, undivided, flat: bracteas lincar.

\section{PASTIN $\Lambda C A$.}

I L. simply pinnate: downy beneath.

\section{IIERACLEUM.}

1 L. pinnate: leatl. pinnatificl, cut, scrratel.

\section{TORISLIUM.}

I Partial loractcas about as long as the flowers: leafl. ovatc, cut, crenate: radiant pet. in pairs, witl very unequal lobes. 
CLASS V. PENTANDRIA.

ORDER II. DIGYNIA.

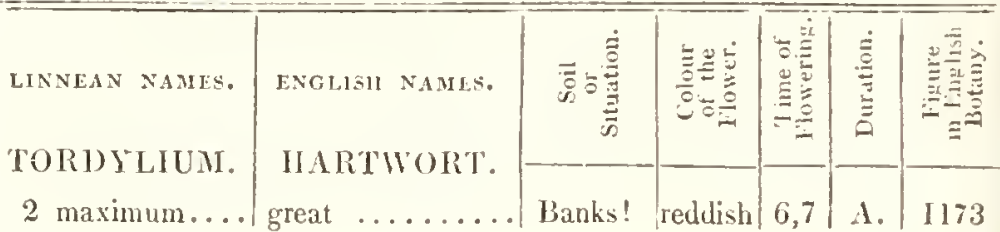

ORDER IH. TRIGYNHA.

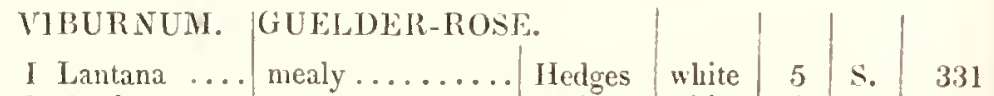

2 Opulu...... comnon........ Mloi. pl. white 6 s. 332

SATIBICUS.

ELDER.

I Ebulus......

2 nigra.......

dwarf .......... Wa. gr.

common ....... Hedges white 6

ST.APIIYLEA. BLADDER-NUT:

1 pinnata.....

\section{TAMARIX. TAMARES.}

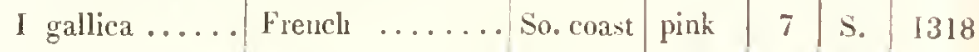

CORRIGFOLA. STRAPWORT,

I littoralis ... sand ........ So. coast $\mid$ white $|7,8|$ A. 668 ORDER N. TETRAGYNA.

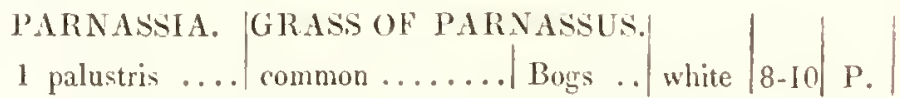

ORDER V. PENTAGYNH.

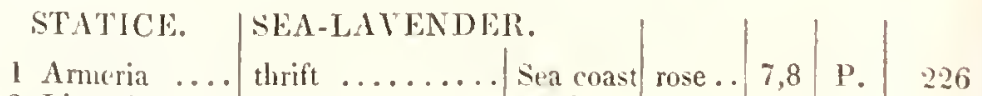

2 Limonium .. common ....... Mtid.sl. blue.. 7,8 P. 102

\begin{tabular}{ll|l|l|l|l|r}
3 reticulata.... & matted $\ldots . . .$. & Mud.sl. & blue.. & 7,8 & P. & 328
\end{tabular} 
SILCIFIC CIIARACTIR.

TORDYLIUM.

2 Leatl. fanceolate, deeply serrated and notched: Fl. somewhat radiaul; outermost pet. of 2 equal lobes: stem rough with close deflexed bristles.

\section{ORDER III. TRIGINIA.}

174. VIBURNUM. Caprifoliacer. Iuss.

I L. cordate, serrated, veiny, tomentous beneatl.

2 L. lobed: petioles glandular.

175. SAMBUCUS. Caprifoliacer. Trss.

1 Cymes 3-parted: stipula leafy: stem herbaceous.

2 Cymes 5-parted: leafi. ovate, serrated: stipula obsolete.

176. STAPHILEA. Celastrint. R. Br.

1 L. pinnate: styles and rapsules 2-together.

177. TAMARIS. Portulaced. Jiss.

1 Fl. pentandrous: lateral clusters numerous: L. lanceolate, spurrerl, acute: branches smooth.

178. CORRIGIOLA. Ilevemea. R. Bis.

I intems inumerous, prostrate.

\section{ORDER H. TEIRAGYNIA.}

179. PARNASSLA. Smifiugra. Juss.

I L. cordate: Nectaries many-bristled.

\section{ORDER I. PENTAGYNIA.}

180. STATICE. Plumbuginet, Juss.

1 scape simple, headed: L. linear: calyx-awns minute.

2 scape panicled, cylind: spikes level-topped: 1. smooth, nerveless, awnerl at the apex.

3 Sicape panicled, prostrate, zigzig, donted; lower branches bancan: L.. werdge-slaped, alwnless. 
CLASS V. PENTANDRIA.

ORDER V. PENTAGYNTA.

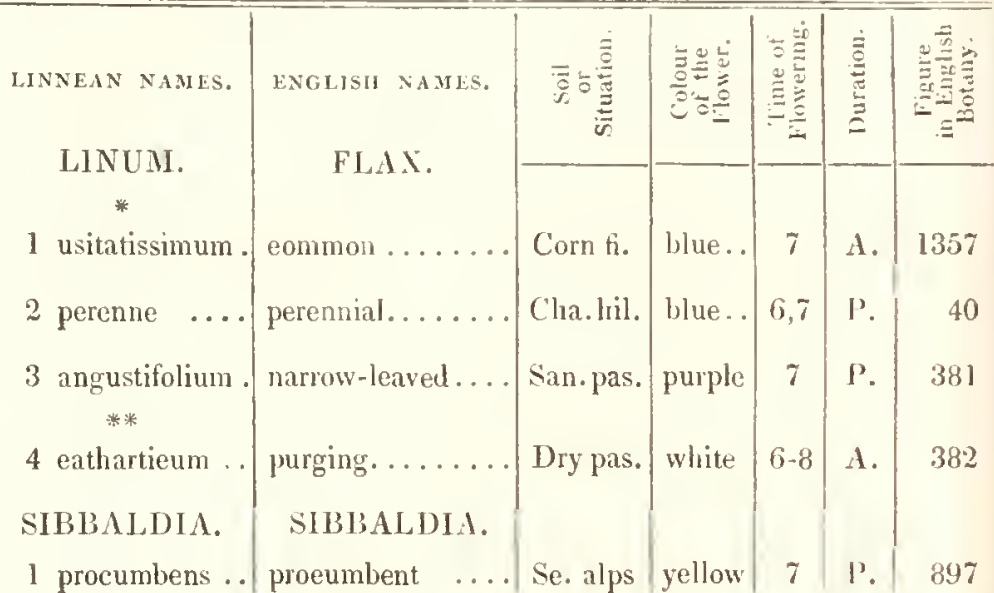

ORDER VI. HEXAGYNIA.

\section{DROSERA. SUN-DEW.}

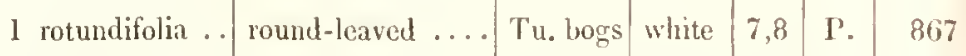

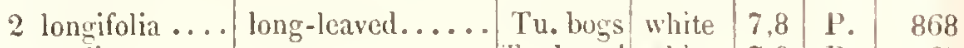

3 anglica..... great ........ T'u. bugs! white 7,8 P. 869

ORDER VII. POLYGYNLA.

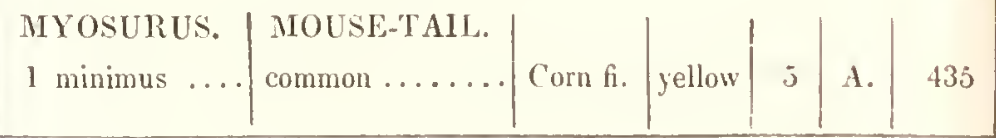


181. LINUM1. Linea. DE C.

* Leaves alternate.

1 Lcat. of the cal. ovate, acute, 3-nerved: pet. crenatc: L. lanccolatc, alternate: stem mostly solitary.

2 Leaf. of the cal. obovate, obtuse, about 5 -nerved, smooth: stems numcrous, asccriding: L. linear-lanceolatc.

3 Leaf. of the cal. elliptical, about 3 -nerved, acuminate as well as tho capsules: L. linear-lanceolate 3-ncrved: stems numcrous. * Lentes opposite.

4 L. olovate-lanceolate: stcm dichotomous above: petals acute.

182. SIBBALDIA. Rasacea. Juss.

1 Leathets wedge-shaped, 3-toothed.

\section{ORDER V1. HEXAGYN1 .}

183. DROSERA. Droseracea. Juss.

I L. depressed, nearly orbicular, radical; petioles hairy: scape bearing a simple racemc.

2 L. obovate, erect, radical: scape bearing a simplc racemc.

3 L. ollong, obtuse, radical: Fl. with 8-styles; caps. 4-valved.

ORDER VII. POLYGYNIA.

184. MYOSURUS. Rammatacé, Juss.

1 L. entire, lincar. 


\section{CLASS VI.}

\section{HEX A N D R I A.}

\section{()RDER I. MONOGINIA}

* Fl. furmished with caly.i and corolla.

202 Frankenia. Cor.5-pet. Cal. 1-leaved, inferior. Caps. I-celled, many-sceded.

201 13rraeris. Cor. 6-pet. Cal.6-leaved, inferior. Bury 2-sueded. 203 Penurs. Cor. 6-pet. C'al. with 6 deep segments, and 6 intermediate plaits. Caps. 2-celled.

$$
\text { Lythrim } 2 .
$$

*** Fl. without a calur, superior.

186 Leucouum. Cor. 6-pet. bell-shaped. Stam. efjual.

185 Ginantius. Cor. 6-pet; 3 inner petals shorter, emarginate.

187 Narcissus. Cor.6-pet. Nect. bell-sliaped, bearing the petals, inclosing the stamina.

*** $F l$. rithout a calyr, inferior.

197 Convaluaria. Cor. 6-cleft. Berry 3-celled. Stig. 3-corired.

193 Ilracintius. Cor. 6-cleft, with inflater tube. stam. unifom. Cips. 3-celled. Seeds globost.

196 Aspanagus. Cor. 6-parted. Berry 3-celled. Stig. 3.

198 Alidum. Cor. 6-pet. P'et. ovate, sessile. Stam. awl-slraped, fattened. Stig. acute. Seeds angular.

194 Anthericum. Cor. 6-pet. Silam. filiform. Stig. obtuse. Seeds argular.

195 Nartinecium. Cor.6-pet. spreading. Sefds tapering at pach end. Siam. hairy.

191 Onxmogatu. Com.6-pet. Stam. dilated at the base.

192 Scilla. Cor. 6-pet. Stam. filiform.

189 Fritumaria. Cor. 6-pet. each with a nectariferous lrollow at 1 lie base.

190 Truma. Cor. Inell-sliaped, G-pet. Myle O. seds flat.

198 Acorus. Cor. 6-pet. Spualix many-flow. Style 0 . Seeds orate, olilong.

$$
\text { ****Fl. apelalous. }
$$

199 Juress. Cul. 6-leaved. Cor.0. Cups. superior, 3-valved, 3-cenlled, nany-seeded.

200 l.ecuns. Cal.6-lenved. Cm.0. Caps. superior3-valved, 1-celled, 3 -seceled.

$$
\text { l'eplis 1. l'olygonum. }
$$




\section{ORIER II. DIGYNI $\Lambda$.}

204 Oxyra. Cal.2-leaved. l'et. 2. Seed 1, compressed, winged.

\section{ORDER III. TRIGYNIA.}

207 Schfuchzeria. Cal. 0. Pet.6. Caps. 3 inflated. Seeds solitary. 209 Coscuicur. Cal. a spathe. Cor. 6-cleft, with a tube from the root. Caps. 3 , inflated. Seeds numerous.

208 Triglocirs. Cal. 3-leaved. Pet. 3. Caps. opening at the base, with 3 valves.

205 Rumex. Cal. 3-leaved. P'et. 3. Seed I, 3-cornered.

206 Tofieldia. Cal. 3-cleft. Pet.6. Caps. 3. many-seeded.

Elatine.

ORDER IV. POLYGYNIA.

210 Alsma. Cal. 3-leaved. Pet. 3. Caps. numerous, aggregate, 1 or 2 -seeded. 
CLASS TI. HEXANDRIA.

(ORIER 1. MONOGYNA.

$=1$

LINNEAN NAMES.

GALANTIIUS. SNOW-DROP.

1 nivalis ..... common .......

\begin{tabular}{|c|c|c|c|c|}
\hline 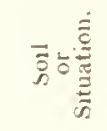 & 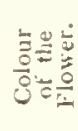 & 它施 & 总 & 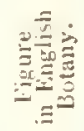 \\
\hline Mead.! & white & 2 & P. & 19 \\
\hline
\end{tabular}

LEUCOJUM.

SNOW-FLAKE.

1 estivum ....

summer ........

\section{NARCISSUS.}

NARCISSUS.

1 poeticus

poetic .........

2 bitlorus......

pale........... San. fi.

3 Pseudo-narcis- daffodil

Hoi.mea!

(white

187 sus

ALLIUM.

\section{GARLICK.}

1 Ampeloprasum great round-hearled Sun. Iril.! purple

2 arenarium ... sand ........ M. woods red

3 carinatum

nor

$$
\text { *** }
$$

4 oleraceum....

5 vineale $\ldots . .$.

****

6 ursinum ....

7 schoenoprasum
FRITHLLARIA. FRITIILARI.

1 Meleayris....

'TULII'A.

I sylvestris .... wild
TULHP. - Woorls yellow

$\mathrm{l}^{3}$.

621

275

276

$4,5 \quad l$.

1657

1358

$7 \quad 1^{\prime}$

1658

1

1088

streaked field .... Corn fi. green

7 P.

488

crow $\ldots \ldots \ldots$......

Chal. so. blish

I?.

1974

broad-leaved ....

Woods

white

5,6

P.

122

Mea.pas.! Hesh 60 l'.

2441

GRITILLAR .....

Moi.pas. purple 4 P.

622

('lualk pits yellow +1 . 
185. GALANTHUS. Amaryllider. R. BR.

l liner pet. tipped with green.

186. LEUCOJUM. Amaryllider. R. Br.

I spatle many-flow. style elub-shaped: pet. uniform dashed with green.

187. NARCISSUS. Amaryllider. R, Br.

1 Spathe 1-How: nect. depressed, very short, membranous, crenate: L. obtusely kccled, margin reflexed.

2 Spathic 2-flow: nect. very short, membranous, crenate: L. 'aeutely kecled, margin inflexed.

3 spathe 1-flow: nect. bcll-shaped, ereet, curled, indistinctly 6-eleft, cqualling the ovate petals.

188. ALLIUM. Asphodelere. R. Br.

* Stem leaves flat.

1 Umbcl globose without bulbs: 3 altcrnate stam. 3-cleft: pet. rough on the back.

2 Umbel bearing bulbs: sheaths cylind: spathc blunt: 3 altcrnate stam. 3-eleft: pet. roughish on the keel.

3 Unbel bulbiferous: stam. all awl-shapcd. spathe aeute.

* Stem leaves culindricul.

4 Umbel bulliferous: L. rough, channelled, furrowed beneath: stam. simplc, awl-shaped.

5 Unbel bulbiferous: 3 alternate stamens 3 -cleft.

**** Scape naked.

6 Scape semicylind: L. lanceolate, petioled: umb. flat-topped: stam. simple.

7 Seape rylindrical, as long as the leavcs: L. cylindrical, somcwhat tapering: stam. simplc.

189. FRITILIARIA. Litiacer. Juss.

1 L. all alternate: stem 1 -flow: nertary linear: pet. points inflexed.

190. TU1.IPA. Liliarea. Juss.

$1 \mathrm{Fl}$. solitary, somcwhat nodding: L. lanceolate: stigma obtuse, triangular: stam. hairy at the basc. 
CLASS V1. HEXANDRIA.

ORDER I. MONOGYNIA.

ORNITHOGALUM. STAR OF BETHLEILE.

l luteum..... yellow......... Woods! yellow

2 pyrenaicum .. tall .......... Pasture! gre-ish

4 P.

$P$.

21

3 umbellatum... common ....... Mea.pas. white

4 nutaus ...... drooping. Dry mea.! white 5

P.

499

HYACINTHUS.

IIYACINTH.

1 racemosus

SQUILL.

I verna vernal.

2 bifolia .

two-leaved ......

3 autumnalis autumnal .

4 nutans la

ANTIERICUM. SPIDER-WORT.

I serotinum... mountain Wel.mo.!

white

6

P.

793

NARTHECIUM.

ASPHODEL.

1 ossifragum. Lancashire .. Tu San. fi.

blue

P.

130

1997

$P$.

ASPARAGUS.

SPERAGE.

1 officinalis common Sen coast gre-ish 
191. ORNITHOGALUMI. Aspholece. R. Br.

1 Stcm angular, 2-leaved: pedunc. umbellate, simple.

2 Raceme very long: filaments all dilated: pcdunc. when in flower, spreading, equal; when in fruit, approaching the scape.

$3 \mathrm{Fl}$. corymbous: pedunc. surpassing the scape: filam. tapering, very entire.

4 Fl. pendulous on one side: filam dilated, cohering, bell-shaped, cloven; alternate ones longer.

\section{SCILLA. Asphodelea. R. Bn.}

1 Bulb coated: corymb. hcmispherical, few-flow: bract. lanceolate, obtuse: L. linear, channellcd, numerous.

2 Bulb coated: Fl. somewhat corymbose. nearly erect, witlout bract: L. generally 2 , lanceolate.

3 L. linear: Fl. somewhat corymbose: pedunc. without bract. ascending, the length of the flowers.

4 L. linear: spike nodding: Fl. drooping, bell-shaped, somewhat cylind. reflexed at the points: bract. in pairs.

193. HYACINTHUS. Aspholeter. R. BR.

I Fl. ovate, 6-furrowed; the upper ones sessile, barren: L. lincar, channclled, loosely spreading.

194. ANTIIERICUM. Asphaleter. R. Br.

1 L. semicylind: those on the stem dilated at their base; stcus mostly I-How.

195. NARTHLCIUM. Juncer. DE C.

I L. ensiform: cluster uninterrupted: bract. one at the basc, the other above the niddlc, of each partial stalk.

196. ASPARAGUS. Asphodeler. R. Bk.

1 Stem herbaceous, unarmed, ncarly crect, cylind: L. setaceous, soft: stipulix mostly solitary.

197. CONVALLARIA. Smilacer. R. Bn.

I Stalk naked semicylind: Fl. spiked, pedicelled, nodding.

2 J. verlicillate, linear-lanceolate. 


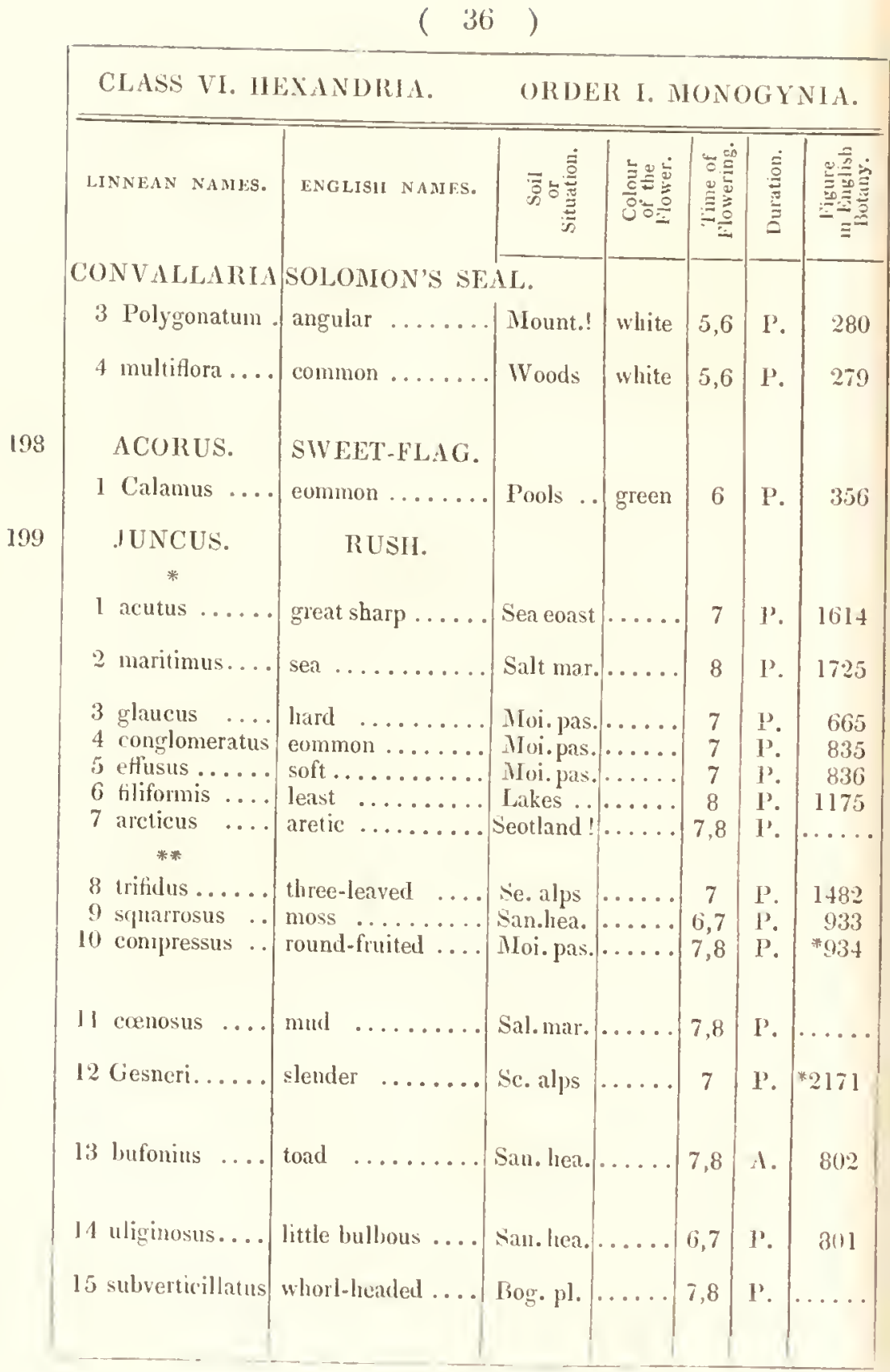


SPECIFIC CIIAACTIR.

CONVALLARIA.

3 l. altcrnate, embracing the stem: stem angular: pedunc. axillary, mostly 1-flow: stam. smooth.

4 I. alternate, embracing the stem: stem cylind: pedunc, axillary, many-flow: stam. downy.

198. ACORUS. Aroider. Juss.

1 scape with a very long leaf-like point above the flowers.

199. JUNCUS. Juncea'. DE C.

* Culms naked.

1 Culm cylind: pan. term: bract. spinours: caps. roundish, bluntly pointed.

2 Culm cylind: pan. term. proliferous: bract. spinous, erect: caps. oblong.

3 Culm straight, glaucous: pan. lateral, erect: caps. elliptical, acute.

4 Culm straight: pan. lateral, conglobatc: caps. retuse: Fl. triandrous.

5 Culm straight: pan. lateral, loose, thrice-compound: caps. obtusc.

6 Culm filiform, rodding: pan. lateral, mostly simple: caps. roundish.

7 Culm straight: pan. near the summit, few-How : caps. oblong.

** Culms leafy.

8 Culm terminated by 3 flowers and 3 bracteas.

9 L. radical, channelled: pan. term. compound, with cymose branches.

10 Culm compressed: L. linear, incurved at the edges: pan. cymose, term: caps. obovate, longer than the obtuse calyx. J.bulbosus Eng. Bot.

11 L. linear, channelled: pan. cymose, term. caps. obovatc, as long as thic calyx.

12 L. slightly channclled: pan. forked, mcemose: calyx-leaves lanccolatc, taper-pointed, 3-ribbed, longer than the caps. J.gracilis. Eng. Bot.

13 L. angular, channelled: pan. forked, raccinose: calyx-leaves lanceolate, taper-pointerl, membranous, 2-ribbed, longer than the capsule.

14 C'ulm bulbous at the base: L. setaceous, channelled: heads lat. and term. about 3-flow. eaps. oltuse, rather longer than the calyx.

15 Cnlm trailing: L. sètaccous, channelled, slightly jointed : pan. forked: heads lat. and term. about 5-flow. whorled: eaps. obtuse, rather longer than the calyx. 
CLASS VI. HFXANDRTA.

ORDER I. MONOGYNHA.

\begin{tabular}{|c|c|c|c|c|c|c|}
\hline LINNLAN NAMES. & ENGLISI NAMES. & 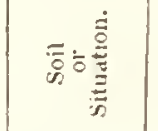 & 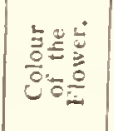 & 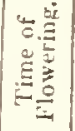 & 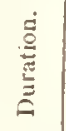 & 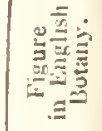 \\
\hline $\begin{array}{c}\text { JUNCUS. } \\
16 \text { capitatus.... }\end{array}$ & $\begin{array}{c}\text { ROSll. } \\
\text { dense-headed .... }\end{array}$ & san. gr.! & & $5-7$ & $\Lambda$ ? & \\
\hline 17 biglumis .... & two-flowered .. & Alp. rills & & 8 & P. & 398 \\
\hline 18 triglumis .... & three-flowered.... & Alp. rills. & & 7 & P. & 899 \\
\hline 19 castaneus... & clustered alpine. & Sc.Hlighl. & & 6,7 & P. & 900 \\
\hline 20 acutiflorus & sharp flowered .. & Moi.pas. & & 6 & P. & 2143 \\
\hline 21 lampocarpus.. & shining-fruited & Moi.pas. & & $6-8$ & P. & 2143 \\
\hline 22 obtusiflorus .. & blunt-flowered .. & Marshes & & 7,8 & $P$ & 2144 \\
\hline 23 polycephalus. . & many-headed .... & Sc. alps & & & P. & \\
\hline $\begin{array}{l}\text { LUCIOLA. } \\
1 \text { pilosa } \ldots . . .\end{array}$ & $\begin{array}{l}\text { WOOD-RUSH. } \\
\text { hairy } \ldots \ldots \ldots \ldots\end{array}$ & Groves & & 3,4 & $P^{\prime}$. & *736 \\
\hline 2 Forsteri .... & narrow-leaved... & Gr.Thick. & & 5 & P. & ${ }^{*} 1293$ \\
\hline 3 sylvatica .... & great & Woods & & 5 & P. & *737 \\
\hline 4 campestris .. & field $\ldots \ldots \ldots$ & Bar. past. & & 4,5 & P. & $* 672$ \\
\hline 5 congesta $\ldots$. & many-headed .... & Tu. bogs. & & 6 & P. & \\
\hline 6 spicata ..... & spiked.......... & Sc. alps & & 7 & P. & $\$ 1176$ \\
\hline 7 arcuata..... & curved......... & Sc.nou. & & 7 & P. & $\cdots \cdots$ \\
\hline $\begin{array}{l}\text { BERBERIS. } \\
\text { I vulgaris ... }\end{array}$ & $\begin{array}{l}\text { BARBFRTY } \\
\text { common ....... }\end{array}$ & Bushy pl. & yellow & 5.6 & s. & 49 \\
\hline
\end{tabular}




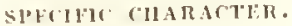

\title{
JUNCUS.
}

6 L. setaceous, channcllerl: heads 1-2. lat. and tem: stam. 3: cal. keeled, bristle-pointed, twice as long as the capsule.

7 Culm simple: 1. Hat: heals solitary of 2 unilateral flowers, surmountel by a leafy bractea.

8 Culm simple: I. flat: heads solitary, term. 3-flow. with oblong braeteas.

9 Culm simple: L. keelerl, flat, sheathing at the base: hearls term. mostly in pairs, many-flow: with leafy loract: eaps. Iwice the length of the calyx.

to 1.. knotty-jointed, somewhat compressed : culm jointless : pan. repeatedly forked, dense: cal. leaves sharp pointed.

21 L. knotty-jointed, complessed : eulm jointless: pan. erect, eompound forked: inner calyx-leaves bordered: caps. coloured. polished.

22 L. and stem knotty-jointed, cylind: pan. divaricated: eal. leaves blunt, as long as the capsule.

23 1. jointed, awl-shaped: pan. ereet, twice or thrice forked: heads many-How: calyx-leaves lanecolate, icute, shorter than the cap. sule.

\section{LUCIOI.A. Juneer. DE C.}

1 1. hairy: pan. cymose, divarieated: Fl. lateral, solitary: eaps. pointed: crest of the seeds looked.

2 Pan. cymose, erect: Fl. solitary: eaps. pointed: crest of the seeds straight, obtuse.

3 Pan. cymuse, deeompound: Fl. and braet. aggregate: eaps. pointed: erest of the seerls obsolete.

4 Pau. of 3 or 4 . partly stalked clusters: eaps, obtuse, with a small point, shorter than the calyx: seeds stalked, without a crest: L. Hat.

5 Pan. of numerous clusters, partly stalked; caps. obtuse, with a small point, as long as the ealyx: seeds stalked, witlrout a crest: 1.. flat, rough-edged.

6 Pan. dense, compound, lobed, drooping: caps. oblong, with a small point: rrest of the seeds obsolete: L. of the stem channellet.

7 Pan. somewlat umbellate, partly compound, with drooping branehes: heads globose, few-flow: bract. membranous, fringed: caps. oblong: L. channelled.

201. BliRBlisis. Berberider. Vext.

J Fil. in pendulous clusters; pet. entire: spines 3 -cleft: scrratures of the leaves brisily. 


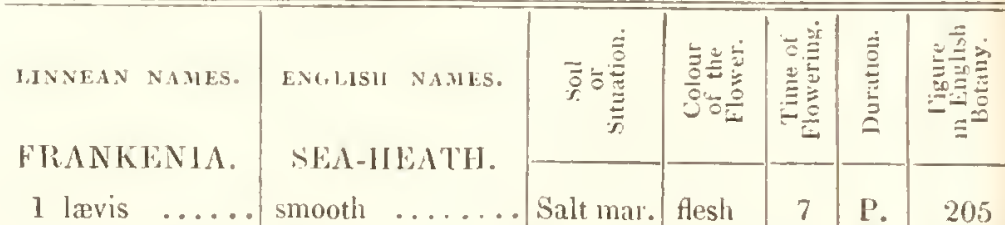

2 pulverulenta.. powdery ....... Sea coast! rerldish 7,8 S 2222 PEJIIS. PURSLANF.

1 Portula .... water ......... Wat. pl. red .. 7,8 A. 1211

\section{ORDER 1I. DJGYNIA.}

OXYRIA. MIOUNTAIN-SORRFI.

I reniformis.... kidney-shaped ..|Alp.bog green $|6| \mathrm{P} . *^{*} 910$

ORDER III. TRIG Y'NIA.

\begin{tabular}{|c|c|c|c|c|c|c|}
\hline 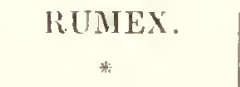 & )OCK. & & & & & \\
\hline I sanguineus .. & bloody-veined.... & Sha. pla. & $\ldots \ldots$ & 7 & $I^{\prime}$. & 1533 \\
\hline 2 crispus...... & curled......... & Rubhle & $\ldots \ldots$ & 6,7 & P. & 1998 \\
\hline 3 acutus ..... & sharp $\ldots \ldots \ldots$ & Wat. pl. & $\ldots \ldots$ & 7 & $l^{\prime}$. & 724 \\
\hline 4 obtusifolius .. & broad-leaved .... & Rubble & $\ldots \ldots$ & 7,8 & '?. & 1999 \\
\hline 5 pulcher...... & fiddle ........ & Grav.pa. & $\ldots \ldots$ & 8 & P. & 1576 \\
\hline 6 maritimus.... & golden......... & Salt mar. & $\ldots \ldots$ & 7,8 & l'. & 725 \\
\hline 7 palustris .... & yellow marsh .... & Marshes & $\ldots \ldots$ & 7,8 & l'. & 1932 \\
\hline 8 Hydrola pathum & great water... & Riv, han. & $\ldots$ & 7.8 & $\mathbf{I}^{\prime}$. & 2104 \\
\hline 9 Acetosil .... & commousoret . & Mca. pas. & & 6 & l'. & 127 \\
\hline () Acotosella .. & sheep's sorrel .... & Grave pa. & & 6,7 & I'. & 167.1 \\
\hline IOFLELI)I A. & TOFl $\mathrm{Fl}, 1) 1 \mathrm{~A}$. & & & & & \\
\hline 1 polustio & S'reottislı & Bog ond1. & gr'l'-ish & 8 & 1'. & $3: 3$ \\
\hline
\end{tabular}


202. FRANKENIA. Fronkeniacer. Sr. Hulani:

'1 L. linear, revolute, crowded, ciliate at the base : Fl. solitary.

2 L. ohovate, abrupt, villous and mealy bencath.

203. PEPLIS. Salicaria. IUss.

1 L. opposite, obovate, stalkerl: pet. wanting, or searcely visillle.

\section{ORDER II. DIGYNIA.}

\section{OXYRIA. Polygomer. Iuss.}

1 L. mostly radical, with ribs radiating from the petiole: Fl. whorlcd, drooping.

\section{ORDER III. TRIGYNLA.}

\section{RUMEX. Polygonea. Iuss.}

* Flouers hermaphrodite.

1 Valves entirc, oblong, one at least bearing a grain: L. cordate-lanccolate.

2 Valves ovate, entire, all grained: L. lanceolate, undulated, acute.

3 Valves oblong, somcwhat toothed, all graincd: 1. cordate-oblong, acuminate: racemes lcafy.

4 Valves toothed, one chiefly grained: radical leaves cordate, obtuse: stem rather rough.

5 Valves toothed, one chiefly grained: radical leaves fiddle-shaped: stem smooth, divaricated.

6 Valves deltoid, fringed with bristly teeth, grained: L. linear; whorls crowded.

7 Valves lanceolate, grained, toothed at the hase: L. linear-lanecolate, whorls distant.

8 Valves ovate, cntire, indistinctly graincd: L. lanceolate, acute.

$$
\text { ** Flovers diacious. }
$$

9 L. ollong, arrow-shaped: valves grained.

10 1. lanceolate, hastate: valves grainless.

206. TOFILIDIA. Melmutherer. R. Br.

I Terak obovate, obtuse. 


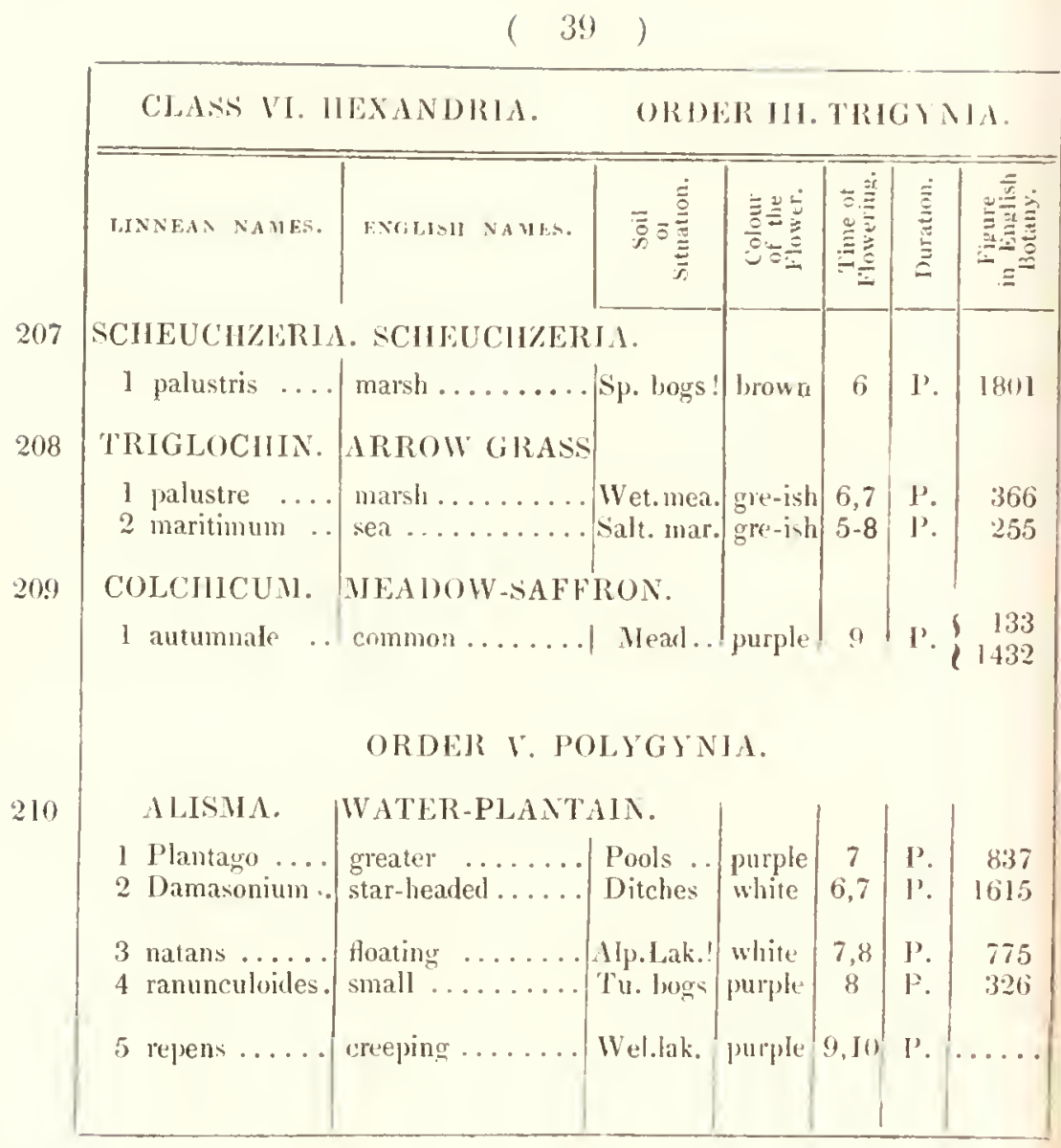


SPECHIC CHARACTER.

207. SCHEUCHZERIA. Juncugineu. Richard.

208. Triglochin. Juncuginer. Richarn.

I Caps. 3-celled, almost lincar, tapering at the base: root fibrous.

2 Caps. 6-cellcd, ovate: root somewhat woody.

209. COLCHICUM. Melanthaces. R. Br.

I L. Alat, lanceolate, erect: segment of the corolla oblong.

\section{ORDER I. POLYGYNIA.}

210. ALISMA. Alismacer. Juss.

I L. ovate, acute: caps. obtuscly triangular.

$: 2$ L. cordatc-oblong: Fl. with 6-styles: caps. 6 , awl-shaped, spreading in a star.

:3 L. clliptical, obtuse: pedunc, solitary: caps, striated.

4 L. linear-lanceolate: caps. 5-cornered, incurved, collected into globular heads: stem none,

5 L. lanceolatc: caps. compressed, acute, numerous, in a globular licad: stcms prostrate, creeping. 



\section{CLASS VII.}

\section{HEPTANDRIA.}

\section{ORDER I. MONOGINIA.}

П1 Trifutans. Cal. 7-leaved. Cor. T-parted, equal, flat. Caps. 7 -valved. Teeds funicated. 


\begin{tabular}{|c|c|c|c|c|c|c|}
\hline \multicolumn{2}{|c|}{ CLASS VII. HEPTANIDRIA. } & \multicolumn{5}{|c|}{ ORDER I. MONOGYNIA. } \\
\hline LINNEAN NAMES. & ENGLISII NAMES. & 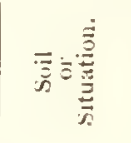 & 题焉 & 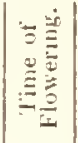 & 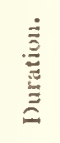 & 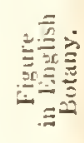 \\
\hline $\begin{array}{l}\text { TRIENTALIS. } \\
\text { I europar .... }\end{array}$ & $\begin{array}{l}\text { TRIENT } \Lambda \text { LIS. } \\
\text { chickweed .... }\end{array}$ & Nor,wds. & white & 5,6 & l'. & 15 \\
\hline
\end{tabular}


$40 \quad)$

CLLASS VII. IIEPTANDRIA. ORDER I. MONOGYNIA.

SPECIFIC CHARACTER.

211. TRIENTALIS. Primulacea. Vent.

1 L. obtuse, obovate-oblong; the lowermost very obtuse. 



\section{CLASS VIII.}

\section{OCTANDRIA.}

\section{OIDDIR I. MONOGINLA.}

* Fl. complete.

no Ackr. Pet.5. Cal.5-cleft, inferior. Caps. 1-seeded, winged. :1S Eprlobius. Pet. 4. Cal. 4-cleft, superior. Caps. 4-celled. Seeds fenthered.

:12 Oevothera. Pet. 4. Cul. 4-cleft, superior. Caps. 4-celled. Seed naked. Anth. linenr.

14. Curona. Cor.8-parted. Cal. 8-leaved, inferior. Caps. 1-celled. :15 Vaccriva. Cor.1-pet. Cal.4-toothed, superior. Seed vessel a Berry.

16 Mrxirsia. Cor.1-jet. Cal,1-leaverl, inferior. Seed vessel a Capsule.

$\because 18$ Ennca. Cor. 1-pet. Cal.4-leaved, inferior. Seed vessel it Capsule. "17 Calluxa. Cor.1-pet. Cal. double: encli of 4 leaves, inferior. Seed vessel a C'apsule.

Monotropa.

** Fl. apetalons.

¿19 Daprine. Cal.col,4-cleft, inferior. Berry 1-seeded.

(DIGYNIA.)

Polygonum. Chrysosplemium. Scierunthus.

ORJ)ER 11I. THIGYNIA.

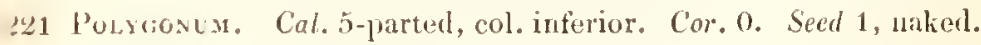

ORDER IV. TETRAGYNIA.

"zy3 Anoxa. Cal. lualf-inferior. Cor. 4 or b-cleft. Caps. covered with the callyx. Seeds 4-bordered.

Eye l’uns. Cal.4-leaved. I'el.t. Berry superior. Seeds numerous, rlobose.

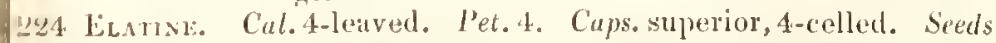
oblong. 
CLASS VIII. OCTANDRIA.

ORDER I. MONOGINIA.

OENOTHERA.

L

LINNEAN NAMES.

ENGLISII NAMES.

1 biennis .... common.......

EVENING
PRIMROSE.

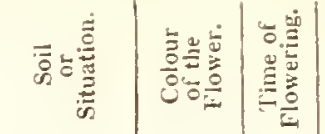

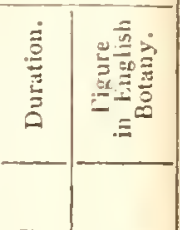

San. sh. yellow $7,9 \quad \mathbf{B}$

1534

EPILOBIUA. WILLOW HERB

*

1 angustifolium

rose bay ...........

Mead... red pur

7,8

P.

19.47

\begin{tabular}{l|l|}
2 hirsutum .... & great hairy ...... \\
3 parviflorum... & small flowered ...
\end{tabular}

Wat. pl.

.

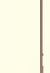

5 roseum $\ldots .$.

broad smooth.... D

Wat.pl.

pink

7 P.

838

6 tetragonum ..

pale smootl ....

Marshes! rose .. 7

P. 693

7 palustre ....

square stalked ..

Marshes purple 7

P. 1948

8 alsinifolium..

marsh .........

Marshes purple 7

P. $\quad: 316$

P. 2000

9 alpinum ....

alpine .........

Alp.riv. red...

6,7

$I^{3}$

2001

\section{CHLORA. YELLOW WORT.}

1 perfoliata... common ....... Chal. so. yell . $v 7,8$

A.

60

VACCINIUM.

BILBERRY.

1 Myrtillus....

bleaberry .......

2 uliginosum ..

great

(1)

lleaths

red .

M. heith flash . 4,5

$\$$.

4.56

S.

581

3 Vitis Idiea ..

cowberry

MI.leith flesh.

S.

508

+Oxycoccus..

cranberry ......

'Tu. bogs rose.. 
SPECIHIC CHARACTER.

212. OENOTHERA. Onagraria. Juss.

1 L. orate-lanceolate, flat: stem rough, somewhat hairy: stam. regnlar: pet. undivided.

213. EPILOBIUM. Onagraria. Juss.

$$
\text { * Fl. irregular. }
$$

1 L. scattered, linear-lanceolate, veined, smooth: Fl. unequal: stam. declined.

$$
\text { ** Fl. regular. Stig. deeply 4-cleft. }
$$

2 L. half embracing the stem, ovate-lanceolate, hairy : stem much branched, root creeping.

3 L. sessile, lanceolate, pubescent: stem nearly simple, woolly : root fibrous.

4 L. petioled, ovate, toothed: stem cylind.

$$
\text { *** Fl. regular. Stig. undivided. }
$$

5 L. petioled, orate, toothed; stem erect, obscurely 4-ingled.

6 L. lanceolate, sessile, finely toothed: stem square.

7 l. sessile, lanceolate, somewhat denticulated: stem cylind.

8 L. petioled, ovate, acute, toothed : root creeping : stem decumbent, 4-ingular.

9 L. opposite, elliptic-lanceolate, obtuse, mostly entire: stem decumbent at the base: few-flow.

214. CHLORA: Gentiana. Juss.

1 L. perfoliate : pan, forked, many-flow. elegant.

\section{VACCINIUA. Vaccinier. De.}

\section{* Leaves deciduous.}

1 Pedunc. 1-flow: J. ovate, serrated: stem angular: cal, wavy, nearly entire.

2 Pedunc. 1-flow : L. obovate, very entire, smooth: branch cylind.

$$
\text { * Leaves evergreen. }
$$

3 Racemes term. nodding: L. obovate, revolute, denticulated, dotted beneath: cor. bell-shaped.

4 L. ovate, very entire, revolute, acute: stems creeping, filiform, snooth : Fl.torm: cor. deeply l-cleft. 
CLASS VIII. OC'TANDRIA.

ORDFR I. MONOGYNIA.

-

LINNEAN NAMES.

(2)

\begin{tabular}{|c|c|c|c|c|}
\hline 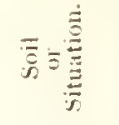 & 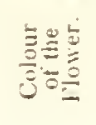 & 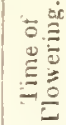 & $\stackrel{\Xi}{\mathscr{E}}$ & 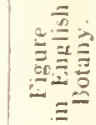 \\
\hline leaths! & & 6,7 & S. & 2. \\
\hline
\end{tabular}

MENZILSIA.

1 cirrulea .... Scotrish .......

2 Polifolia .... Jrish

Nount.

\section{CALLUNA.}

LING.

1 vulgaris

.

common ........

POLYGONUM. JPSRICARIA.

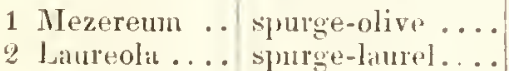

HEATII.

1 Tetralix cross-leaved ....

2 cinerea...... fine-leaved ......

3 vagans......

Cornish

4 ciliari

ciliated

\section{MEZFREON.}

\section{1) $\triangle$ PHNE。}

Woods!

Woods

rose. . 3,4 green :

S.

1331

s.

11!

\section{ACliR.}

[nus $\}$

1 Pseudo-platia- sycamore .....

2 cimpestre... con

\section{MIAPLE.}

cycimore ..... lledges

grienen

srreen 5,6

S. * 1013

S.

101.1

101.3

s.

\begin{tabular}{ll|l} 
Boggygr. & red $\ldots$ & 0,7
\end{tabular}

i)

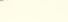

\section{ORDER HI. TRIGYNIA.}
1 anphibium ..
2 Persicurial...
anuphibions. ....
Ditches
\begin{tabular}{l:c}
$\operatorname{ros}{ }^{2}$ & 7,8 \\
\hline $\operatorname{rose} \ldots$ & 7,8
\end{tabular}
\$ lapmothifolium.
pale-flowemol.... Junghill gre-ish i,8
4. Hydropipure..

P.

436

d. $7(i$.

1. 1:38: 
216. MFNZIESIA. Ericer. Juss.

1 L. linear, obtuse, with cartilaginous teeth: jedunc. term. nggrogate, simple: Fl. 5-cleft, decandrous.

2 L. ovate, revolute, downy beneatli : racemes tem. leafy, mayHow. Ericu Daboci, Eng. Bot.

217. CALLUNA. Ericea. Juss.

1 L. arrow-shiaped, closely imbricated: Fl. in long, unilateral clusters. Erica rulgaris, Fing. Bot.

\section{ERICA.}

1 Anth.horned : style nearly concealed : cor. ovate: L. in fours, ciliated : Fl. in round heads.

2 Antl. crested : style a little protruded: stig. capitate : cor. ovate: L. in threes.

3 Anth. simple, deeply cloven; protruded as well as the style: cor. bell-shaped: L. in fours: Fl. axillary, crowded.

4 Anth. simple, bificl, included: cor. ovate, inflated: L. 4 in a whorl, ciliate, glandulose: FI. in terminal unilateral ricemes.

219. DAPHNE. Thymelex. Juss.

1 Fl. sessile in threes on the stems: L. lanceolate, deciduous.

" Racemes ixillary, about 5-flow. draoping: L. lanceolate, smootl, evergreen: cal. obtuse.

220. ACER. Acerined. Juss.

1 L. 5 -lobed, unequally serrated: clusters pendulous.

2 L. b-lobed, obtuse, notched : clusters corymbose, erect.

\section{ORDER III. TRIGYNIA.}

221. POLYGONUM. Polugoned. IUSS.

* Digynous.

1 Fl. pentandrous, styles 2, united half-way ul' : spike ovite.

2 Fl. lexandrous: strles 2, united half-way up: spikes orits-oblong, erect: perlunc. smooth: stip. fringed.

3 Fl. hexandrous: styles distinct : perlunc, rough : stip. not fringed : seeds concave on both sides.

4 Fl. hexindrous: styles 2, uniterl half-way up : L. linceolate, undulated, spotless: clusters lax, nodding : stem croct. 
CLASS VIII, OCTANDRIA.

ORDER III. TRIGYNIA.

\begin{tabular}{|c|c|c|c|c|c|c|}
\hline LINYEAN NAMES. & ENGLISH NAMES. & 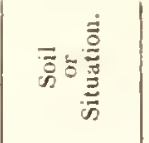 & 部密 & 证产 & & 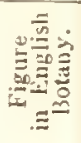 \\
\hline 5 minus ..... & small creeping .. & Wat. com & red .. & 9 & A. & 10.13 \\
\hline 6 Bistorta .... & snakeweed...... & Mea.pas. & rose.. & 6 & I'. & 509 \\
\hline 7 viviparum .. & alpine bistort... & Alp.pas. & rose.. & 6,7 & P. & 669 \\
\hline 8 aviculare .... & knotgrass $\ldots . .$. & Rubble & gre-ish & $4-10$ & $\Lambda$. & 1252 \\
\hline 9 Fagopyrum 9. & buck-wheat ... & Corn fi. & flesh & 7,8 & A. & 1014 \\
\hline 10 Convolvulus & black bind-weed & Corn fi. & white & $6 \cdot 8$ & A. & 941 \\
\hline \multicolumn{7}{|c|}{ ORDER IV. TETRAGYNIA. } \\
\hline $\begin{array}{c}\text { PARIS. } \\
1 \text { quadrifolia .. }\end{array}$ & $\begin{array}{l}\text { HERI PARIS. } \\
\text { common....... }\end{array}$ & Itoods & green & 5 & P. & 7 \\
\hline ADOXA. & MOSCHATELL. & & & & & \\
\hline 1 Moschatellina & tuberous........ & Sha. jl. & green & $\cdot 1,5$ & I. & 4,53 \\
\hline ELATINE. & WATERWOR'T. & & & & & \\
\hline 1 tripetala .... & small ......... & Mar.Lak! & white & 7,8 & A. & 955 \\
\hline
\end{tabular}




\section{SPEEIFIC CIIARACTER.}

\section{POLTGONUM.}

5 Fl. hexandrous: styles eombined: L. linear-lanceolate, fat: elusters lax, filiform, almost ereet: stem trailing at the base.

\section{* Trigynous.}

6 Stem quite simple, single spiked: L. ovate, undulated, decurrent into the petiole.

7 Stem quite simple, single spiked: L. lanceolate, margin revolute.

8 Fl. axillary: L. elliptic-lanceolate, margin rough: nerves of the stip. remote: stems procumb. herbaeeous.

9 L. heart-arrowshaped - stem almost upright, smootlı: angles of the seeds equal.

(1) L. heart-arrowshaped: stem angular, twining: segments of the calyx obtusely keeled.

\section{ORDER IV. TETRAGYNIA.}

222. PARIS. Smilucer, R. Br.

I Stem simple: 1. four, ovate: Fl. solitary.

223. ADOXA. Saxifragea. Juss.

I L. hi-ternate, lobed.

224. FLATINE. C'aryophyllear. Juss.

I L. in pairs, rough with minute points: Fl. mostly 3-cleft. 



\section{CLASS IX.}

\section{EN NEA N D R I A.}

\section{ORDER I. HEX゙AGYNIA.}

25 L'vтомus. Cal.0. P'et. 6. Caps. 6, many-sceded. 


\begin{tabular}{|c|c|c|c|c|c|c|}
\hline \multicolumn{2}{|c|}{ CLASS IX. ENNEANDRIA. } & \multicolumn{5}{|c|}{ ORDER I. HEXAGYNIA. } \\
\hline LINNEAN NAMES. & EXGLISI NAMES. & 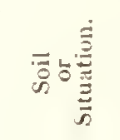 & 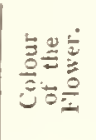 & 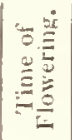 & 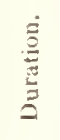 & 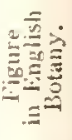 \\
\hline $\begin{array}{l}\text { BU'TOMUS. FLO } \\
1 \text { umbellatus . }\end{array}$ & $\begin{array}{l}\text { WERING RUSH } \\
\text { common ........ }\end{array}$ & Ditches & rose.. & 6,7 & $1^{\prime}$. & 651 \\
\hline
\end{tabular}


SHECITIC CIARACTER.

225. BUTOMUS. Butomere. Ricinard. 


\title{
CLASS $X$.
}

\author{
D E C A N D R I A.
}

\section{ORDER I. NIONOGYNIA.}

* Fl. many-petalled.

226 Monotropa. Pet. 8 or $10 ; 4$ or 5 outer unes gibbous at the base. 229 Pyrola. Pet. 5. Antherce 2-eelled, with 2 -pores.

$$
\text { * Fl. 1-pet. equal. }
$$

227 Andromeda. Cor. ovate. Caps.5-celled.

228 Aniutus. Cor. ovate, pellucill at the base. Berry 5-cellerl. Vacrinium 1, 2. Mensiesiu 1.

\section{ORDER II. DIGYNIA.}

232 Sclemantius. Cor. 0. Cal. 1-leaved. Seeds 2.

230 Cimsosplexium. Cor.0. ('al.coloured. Caps.2-beaked, manyseeded.

231 saxilraci. P'et.5. C'al.5-parted. Cups. 2-beaked, many-seeded. 233 Saposaria. P'et.5. Cal. I-leaved, tubular, without angles, naked at the base. Caps. oblong.

234 Diantinus. Pet. 5. Call. 1-leaved, tubular, scaly at the base. Caps. oblong.

\section{ORDER III. TRIGYNIA.}

237 Arenaria. Caps. 1-celled. P'et. entire, spreadung.

236 Strelania. Cips. 1-celled. Pet.2-parted, spreading.

238 Cuentina. Caps. l-celled. Necturiferous cilunds 5 at the base of the stimina. Pet. 0.

235 Silene. Cups. imperfeetly 3-celled. P'et. 2-cleft, with claws. Cal. 1 -leaved. 
99 Cotylenos. Caps.5. Nect. of 5 seales at the base of the germen. Cor. 1-pe:.

10 Strum. Caps.5. Nert. of 5 seales at the hase of the grermen. Cor. 5-pet.

1 Oxatrs. Caps. 5-celled, angular. Seeds 2, coated. Pel. united at the base.

3 Licusis, Caps. 5 or 1-celled, many-seeded. Cal. tubular, membranous.

2 Agrostemma. Caps. 1-celled. Cal. tubular, eoriaceous.

4 Cerastium. Caps. 1-celled. Pet. 2-eleft. Cal.5-leaved.

5 Spergula. Caps. 1-celled. Pet. entire. Cal.5-leaved.

Stellaria 6. 7. Adora. Rhodiola. 
CLASS X. DECANDRIA.

ORDER I. MONOG INIA.

LINNFAN NAMES. $\mid$ ENGLISH NAMES.

1 IIypopitys .. yellow.

ANIROMEDA.

1 polifolia...

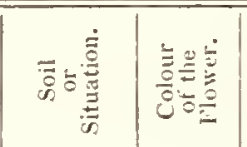

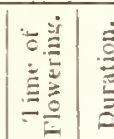

\begin{tabular}{l|l}
\hline P. & \\
\hline & 69
\end{tabular}

ARBUTUS.

marsh ......... Nor. bogs: flesh

1 Unedo......

\section{ARBUTUS.}

strawberry tree ..

2 alpina $\ldots . .$.

black alpine .... Se. moun. white

3 Uva-ursi .... red trailing ..... Alp.hea. flesh

\section{PJTOLA.}

WINTER-GREEN.

1 rotundifolia ..

2 media.... in

3 minor $\ldots . .$.

4 seeunda... s sern intermediate .... Woods! white

\section{ORDIRK II. DIGYNIA.}

230 CHRYSOSPLENIUM. GOLDEN SAXIFRAGE.

1 alternifolium . alternate-leaved .. Wt.sh.p ! yellow

2 oppositifolium. opposite-leaved .. Wet sl.p. yellow

SAXIFRAGA.

\section{SAXIFRAGE.}

1 Geum ....... kidn

kidney-leaved .... 1r. moun. white

2 hirsuta......

lirsute ...... Ir. moun. purple

3 umbrosa I

4 stellaris. Mount. flesh Alp. riv. white $6,7 \mathrm{I}$. 
226. MONOTROPA. Pyroled?

Fl. lateral, with 8 -stamens: term. with 10 . -in a drooping cluster.

227. ANDROMEDA. Ericer. JUSS.

Pedunc. aggregate, term: L. alternate, lanceolate, revolute, glaucous beneath.

228. ARBUTUS. Ericex. Juss.

Stem arboreous: L. smooth, obtusely serrated: pan. term: berrics many-seeded.

2 Stems procumb: L. wrinkled, serrated: clusters term.

B Stems procumb: L. very entire: clusters term.

\section{PYROLA. Pyroler?}

1 Stamens ascending: pistil declining: raceme many-flow : cal. as long as the stamens.

2) Stam inflexed: style curved downwards, clongated : stalk spiral: Fl. pendulous: cal. shorter than the, stamens.

B Stam. inflexed: pistil straight: stig.5-lobed, pointless, without a ring. A Raceme unilateral: style straight: stig. 5-lobed: L. ovate, acute, serrated.

ธ Peduncle 1-flowered: stig. with 5-pointed rays.

ORDER II. DIG Y NIA.

230. CHRYSOSPLENIUM. Sarifragea. Juss.

1 L. alternate.

2 L. opposite.

231. SAXIFRAGA. Saxifrager. Juss.

* Leaves undivided: stem almost naked.

11 L. reniforn, notched: petioles linear channelled, much longer than the leaves: caps. superior.

2 L. oval, with sharp, cartilaginous notches: petioles linear, much longer than the lcaves: caps. superior.

L. olsovate, with sharp, cartilaginous notches; tapering at the base into dilated flat petioles: caps. superior.

:1 L. wedge-shaped, coarsely serrated; tapering and cntire at the base: pan. few-flow: caps. snperior. 


\begin{tabular}{|c|c|c|c|c|c|c|}
\hline LINNEAN NAMES. & ENGLISH NAMES. & 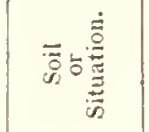 & 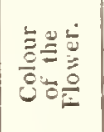 & 焉 & 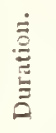 & 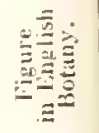 \\
\hline $\begin{array}{l}\text { SAXIFRAGA. } \\
5 \text { nivalis ..... }\end{array}$ & $\begin{array}{l}\text { SAXIFRAGE. } \\
\text { clustered alpinc .. }\end{array}$ & Sc. alps & white & 7 & $P^{\prime}$. & 440 \\
\hline$* *$ & & & & & & \\
\hline $\begin{array}{ll}6 & \text { oppositifolia .. } \\
7 & \text { Hirculus .... }\end{array}$ & $\begin{array}{l}\text { purple ......... } \\
\text { yellow marsh .... }\end{array}$ & $\begin{array}{l}\text { Alp. roc. } \\
\text { Tu. bogs! }\end{array}$ & $\begin{array}{l}\text { purple } \\
\text { yellow }\end{array}$ & $\begin{array}{l}4 \\
8\end{array}$ & $\begin{array}{l}\text { I?. } \\
\text { I?. }\end{array}$ & $\begin{array}{r}9 \\
1009\end{array}$ \\
\hline 8 aizoides $\quad \ldots$. & yellow mountain.. & Alp.riv. & yellow & 7,9 & $P$. & 39 \\
\hline * 类菜 & & & & & & \\
\hline 9 granulata .... & white meadow .. & San. pas. & white & 5 & P. & 500 \\
\hline 10 cernua $\ldots .$. & drooping bulbous . & sc.alps. & white & 7 & $P^{\prime}$. & 664 \\
\hline 11 rivularis $\ldots$. & alpine brook $\ldots$. & Alp. riv. & white & 6,7 & $P$. & 2275 \\
\hline 12 tridactylites .. & rue-leavad ..... & Walls .. & white & 5 & A. & 501 \\
\hline 13 muscoides .. & mossy alpine .... & Nor.mou. & yellow & 5 & P. & \\
\hline 14 pygmas .... & dwarf alpint $\ldots$... & Sc.alps! & yellow & 6,7 & P. & *2314 \\
\hline 15 caspitosa .... & tulted alpine $\ldots$ & Wel. alps & cream & 5,6 & $1^{\prime}$. & 794 \\
\hline 16 hirta....... & hairy alpine $\quad . .$. & Sc. alps & white & 5,6 & $\Gamma^{\prime}$ & 2291 \\
\hline 17 affinis ..... & involute alpine . & lr. mou. & white & 5,6 & P. & \\
\hline 18 platypetala .. & broad-petalled .. & IIa.-Seot & white & 6 & $P$ & 2276 \\
\hline 19 incurvifolia .. & curve-lcaved .... & Ir. moun. & white & $\cdots$ & P. & \\
\hline 20 rlenudata .... & smooth Grampian. & Sc.Iligh. & white & $\cdots$ & $P^{\prime}$ & \\
\hline 21 hyproides.... & mossy $\ldots \ldots \ldots$ & Wal. Kocl & white & 5,6 & P. & 454 \\
\hline 22 elongella .... & long-stalked & s̀c. alp. & wlite & 6 & I'. & 2277 \\
\hline 23 leptopliylla & narrow speatling. & II el.mou. & whitc & 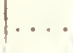 & $1 '$. & \\
\hline
\end{tabular}




\section{SAXIFRAGA.}

5 L. obovate, serrated; tapcring and entire at the basc: cluster capitate, few-flow: cal, spreading: caps. half inferior.

\section{** Leaves undivided. Stem leafy.}

6 Branches 1-flow: L. opposite, imbricated, fringed: pct. ovate.

7 Stem crect: 1. alternate, lanceolate, naked: cal. reflexed, obtuse, friuged : caps. superior.

8 Stem decumbent at the base: L. alternate, lincar, with fringe-like teeth : cal. spreading: caps. half superior: Fl. recept : depresscd.

\section{**** Leaves lobed.}

9 Stem leafy: L. reniform, lobcd: root granulated: germ. half inferior.

.0 Stem with aggregate axillary bulbs: L. palmate, stalked: pct. obovate: germ. superior.

1 Stem few-flow: L. palmate, stalked: uppermost spatulate: root fibrous: germ. half inferior.

$\therefore 2$ L. wedge-shaped, with 3 or 5 segm. uppermost undivided: pedunc. 1-flow. alternatc: germ. inferior.

:3 L. linear, obtuse, smooth, 3 ribbcd: undivided, or with 2 small lateral lobes: pet. nearly linear: cal. almost naked.

4 L. linear abrupt, smooth, undivided: pet. obovate, scarcely longer than the obtuse glandular calyx.

.5 L. radical crowded 3 or 5 -cleft, obtuse, veiny, fringed; lowermost undivided : Fl. 1.5 or more: pet. rounded, 3 -ribbed : cal. obtuse : germ. half inferior, hairy.

6 L. radical rather crowded, 3 or 5-cleft, pointed, vciny, fringed: cal. acute: pet. obovate, 1-ribbed: germ. half inferior.

7 L. 3-5-clcft, lobes linear, pointed : cal. segm. awl-shaped, channelled, pointed, recurvcd: pet. oblong: edges inflexed.

8 L. 3-5-cleft; lobcs bristle-pointed: cal. segm. ovate, pointed, erect: pet. nearly orbicular, flat, with many latcral vcins.

9 L. 3-5-clcft: segm. lanccolate, obtuse, incurved: cal. scgin. ovatc, acute: pet. roundish, slightly cloven.

20 L. 3-5-cleft; segm. awl-shaped, bristlc-pointcd, smooth : cal. scgm. lanceolate, minutely pointed: pet. obovate, cloven.

"1 L. radical 3-5-cleft; uppernost undivided; all bristlc-pointed, fringed: cal. segm. ovate, pointed: pet. obovatc: stig. ncarly smooth.

22 L. 3-5-cleft: bristle-pointed, fringed : pedunc. very long: cal. pointed: pet. obovatc.

23 L. 3-5-cleft, scgm. lincar-lanccolate, acute, widely spreading: cal. ohlong, ovate: pet. spatulatc, undividcd. 
CLAŚS X. DECANDRIA.

ORDER 11. DIGYNLA.

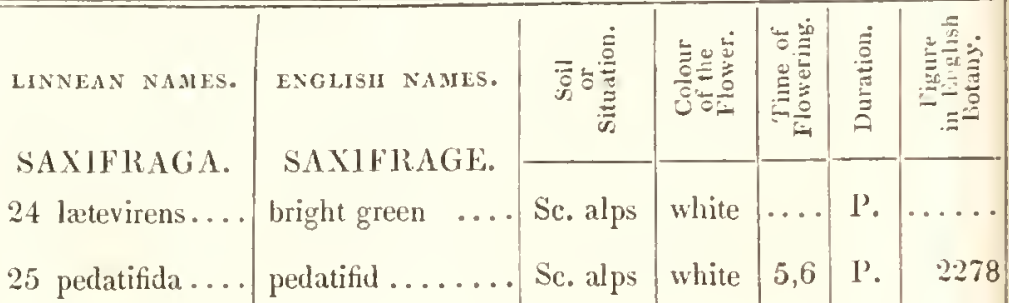

SCLERANTIIUS

1 annuus.....

KNAWEI.

2 perennis .... aninual.........

San fi. perennial San. hea.!

\section{SAPONARIA.}

SOAPIVORT.

1 officinalis.... common....... Hedges

DIANTHUS.

*

1 Armeria...

2 prolifer ........

\section{PINK.}

Deptford....... Grav.pas. red .. 7,8

A.

317

proliferous ...... Grav.pa.! purple

A. 956

\section{***}

3 Caryophyllus.

4 deltoides ....

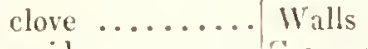

maiden

Grav.pas.

flesh

7

I'. 214

I'.

****

5 casius

\section{ORDER 11I. TRIGYN1A.}

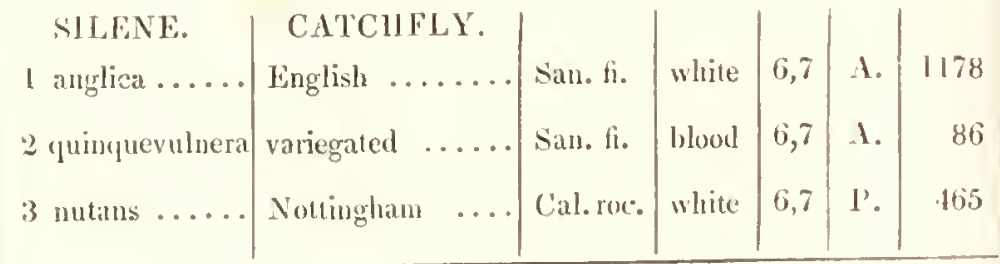


SPECIFIC CIIARACTER.

\section{SAXIFRAGA.}

L. 3-5-cleft: segm. linear, acute: shoots long and trailing: cal. lanceolate, pointed: pet. spatulate, slightly cloven.

L. radical reniform, divided in a pedate manner into 7-lobes: pan. level-topped, many-flow: cal. superior; segm. linear-lanccolate, as long as the capsule.

\section{SCLERANT11US. Illecebrea. M. Br.}

1 Cal. of the fruit spreading, acute: stems spreading.

2 Cal. of the fruit closed, obtuse: stems procumbent.

\section{SAPONARIA. Caryophyllea. Juss.}

II Cal. cylind. L. elliptic-lanceolate.

\section{DIANTHIUS. Caryophyller. Juss.}

\section{* Flowers aggregate.}

1 Fl. fascicled: cal. scales lanceolate, downy, equal to the tube: pet. serrated.

2 Fl. capitate: cal. scales ovate, blunt, beardless, membranous, surpassing the tube.

\section{** Flowers solitary: stem many-flow.}

3 Cal. scales almost rhomboid, very short: pet. crenate, hcardless.

4 Cal. scales mostly 2, ovate-lanceolate, acute: L. rather obtuse, somewhat pubcscent: pet. crenate, smooth.

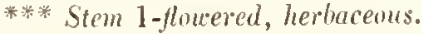

5 Cal. scales roundish, short: pet. crenate, pubescent: L. rough in the margin.

\section{ORDER III. TRIGYNIA.}

235. SILENE. Caryophyller. Juss.

1 hairy, viscid: pet. notched: Fl. lateral, crect, altcrnate: lower ones when in fruit, divaricated or reflexed.

2 liairy: pet. roundish, very eutire: Fl. lateral, alternate, ercet, as well as the capsules: cal. shaggy.

3 Fl. pan. 1-rowed, drooping: pet. cloven, segmeuts linear, with an acute cloven scale: $\mathbf{1}$. lanceolate, pubescent. 
ORDER HI. THIGYNIA.

\begin{tabular}{|c|c|c|c|c|c|c|}
\hline LINNEAN NAMES. & CATCHFLY. & ") & 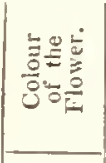 & 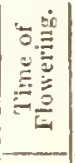 & 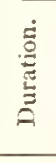 & 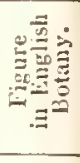 \\
\hline 4 inflata $\ldots .$. & bladder ....... & Pas.Ro.si & white & 7 & P. & $* 164$ \\
\hline 5 maritima .... & sea $\ldots . . . .$. & Sea sli. & white & 8,9 & $P$. & 957 \\
\hline 6 Otites $\ldots .$. & Spanish ....... & Grav.so.! & yell-ish & 7,8 & $\mathrm{I}^{\prime}$. & $\therefore 85$ \\
\hline 7 conica ...... & rorn $\ldots \ldots \ldots$ & San. ti. & rose.. & 7 & A. & 922 \\
\hline 8 noctiflora .... & night-flowering .. & San. fi. & blush & 7 & I. & 291 \\
\hline 9 Armeria $\ldots$. & Lobel's ........ & $\mathrm{Fi} .1 \mathrm{Talls}$ & $\operatorname{rose}$. & 7,8 & A. & 1398 \\
\hline 10 acaulis ...... & $\operatorname{moss} \ldots \ldots \ldots$ & Sc. alps & rose.. & 6,7 & P. & 1081 \\
\hline SFELLARH. & STITCHWORT. & & & & & \\
\hline 1 nemorum .... & wood $\ldots \ldots \ldots$ & Moi.wo. & white & 5,6 & P. & 92 \\
\hline 2 media ...... & com. chickweed .. & Cult. gr. & white & $4-10$ & A. & 537 \\
\hline 3 holostea $\ldots$. & greater $\ldots . .$. & Gr. lled. & white & 5 & $1^{\prime}$. & 511 \\
\hline 4 graminea .... & lesser $\ldots \ldots \ldots \ldots$ & san. so. & white & 5 & $\perp^{\prime}$. & 803 \\
\hline 5 glauca ..... & glaucous marsh .. & Moi.mea. & white & 6,7 & $P$. & 825 \\
\hline 6 uliginosa .... & $\operatorname{bog} \ldots \ldots \ldots$ & Rivulets & white & 6 & A. & 1074 \\
\hline 7 cerastoides .. & alpine ......... & sic. alps & white & 6 & P. & 911 \\
\hline 8 scapigera .... & many-stalked .... & Sc. rivul. & white & 6 & I. & 1269 \\
\hline$A R E N \backslash R I A$. & SANDWOR'. & & & & & \\
\hline 1 peploides .... & & Sea shore & white & 6,7 & $P$. & 189 \\
\hline 2 trinervis $\ldots$. & plantain-leavel .. & Mlo.sli.pl. & white & 5,6 & 1. & 1483 \\
\hline 3 serpyllifolia... & thyme leaved .... & Walls & white & 6,7 & A. & 92.3 \\
\hline 4 tenuilolia .... & fine leaved ...... & San.ti. & white & 6 & A. & 219 \\
\hline 5 verna & vernal. & Mount. & white & $5-8$ & P. & 512 \\
\hline
\end{tabular}


SPECIFIC CIIARACTER.

\section{SILENE.}

4 Fl. pan. drooping: pet. semibifid, almost naked: eal. smooth, with reticulated veins, stcm ereet. Cucubalus Behen, ling. Bot.

ü Fl. mostly solitary, term. pet. bifid, crowned: cal. smooth, with reticulated veins: stem decumb: L. lanceolate.

Fl. pan. dioxcious: pet. linear, undivided, naked: L. spatulate, roughish. Cucubulus, Eng. But..

7 Stem diehot: pet. bifid, crowned: L. soft: cal. in fruit eonical, with 30 furrows.

:8 Stem dichot: pet. eloven, bluntly erowned: eal. with 10 hairy ribs; teeth as long as its tube.

9 Pan, diehot. level-topped, many-flow : pet. notched, acutely erowned: L. smooth: eaps. not longer than its stalk.

$.0 \mathrm{~L}$. linear, eiliated at the base: pedunc. solitary, term. l-flow: pet. crowned: cal. smooth.

\section{STELLARIA. Caryophyllea. Juss.}

1 L. lower cordate, petioled; upper ovate, sessile: pan. diehot.

2 L. ovate: stems procumb. with a hairy, alternate, lateral line.

$.3 \mathrm{~L}$. lanceolate, serrulatel : pet, obcordate: eal. nerreless.

4 L. linear-lanceolate, very entire : pan. term. divarieated: eal. 3-nerved, nearly equal to the petals.

5 L. linear-lanceolate, very entire, glaueous: pedune. erect: cal. 3nerved, shorter than the petals.

6 L. elliptic-lanceolate, very entire, callous at the apex. 17. irregularly panicled, lateral or term : pet. shorter than the calyx.

7 L. elliptic-oblong, obtuse: stems with a laairy lateral line; generally 2-flow: cal. l-nerved, pubescent: pedunc. downy.

8 Stem shorter than the pedunc: L. linear-lanceolate, rough-edged: eal. 3 -nerved, the length of the petals.

237. ARENARIA. Caryphyllea. IUss.

1 L. ovate, acute, fleshy: cal. obtuse, nerveless.

2 L. ovate, acute, petioled, nerved: cal. keel rough, indistinctly 3 nerved.

3 L. ovate, almost sessile, rough : eal. hairy, 3 outermost leaves 5 nerved.

4 L. awl-shaped, acute: stem panieled: caps.erect, 3-valved : pet. lauceolate, shorter than the calyx.

5 L. awl-shaped, rather oltuse: stem panicled: pet, obovate, shorter than the eal: eal. 3-nerved, nerves remote, equal. 
CLASS X. DECANDRIA.

ORDER III. TRIGYNIA.

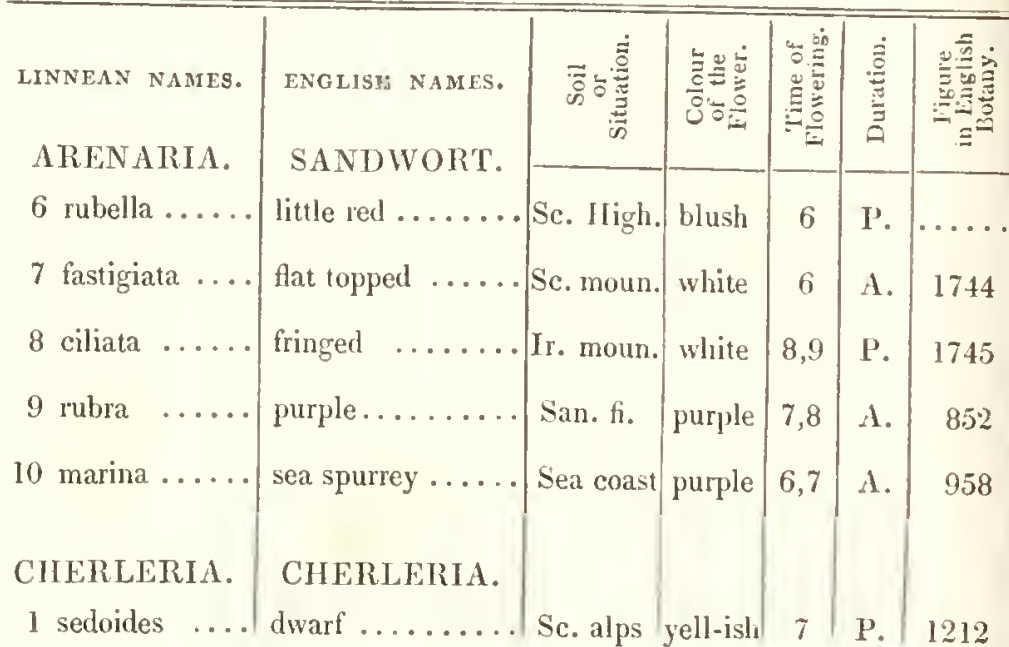

ORDER IV. PENTAGYNIA.

\section{COTYLEDON. NAVELWORT.}

I Umbilicus ..

common ....... Sha. roc. yell-ish 6,7 $\mathrm{I}^{\prime}$.

325

2 lutea........

greater yellow .. Moi. roc.! yellow

6,7

P.

1522

SEUUM.

米

1 Telephium .. **

2 dasyphyllum.

3 anglicum ....

4 acre .......

5 sexangulare..

6 villosum ....

7 albun

\section{STONECLOP.}

Orpine

$$
\text { thick-leaved .... }
$$

English ........

biting

Rocks.. white

Walls .. yellow

Walls! yellow

6,7

1.

hairy

Mo. al.pl. white

6,7

l'.

Rocks!

white 71 1'.

1'. 1578 


\section{ARENARIA.}

3 L. awl-shaped, obtuse: stems 1-flow: ealyxuleaves longer than the petals; with 3 equal ribs.

7 L. awl-shaped: stem ereet eorymbose: pet. very short: eal. nerves lateral, dilated.

\$ L. spatulate, fringed at the base: stems prostrate, branehed downy:

Fl. term. solitary: eal. 5 or 7 -nerved.

9 L. linear, mueronulated: stip. membranous, sheathing: seeds compressed, angular, roughish.

(1) L. semieylind. fleshy, awnless: stip. membranous, sheathing: seeds

eompressed, marginate, smooth.

238. CHERLERIA. Caryophyller. Juss.

ORDER IV. PENTAGYNIA.

239. COTILFDON. Crassulacer. DE C.

1 L. peltate, erenate: stem mostly simple: Fl. pendulous, braeteas entire: root tuberous.

$: 2$ L. erenate-toothed: lower ones peltate: stem spiked, mostly simple:

Fl. erect: root ereeping.

240. SEDUM. Crassulacea. Juss.

* Leaves flat.

1 L. flattish, serrated: eorymb. leafy: stem ereet.

** Leaves cylindrical.

" 2 L. ovate, obtuse, fleshy, sessile; the lower ones opposite: stem weak: pan. glutinous.

3 L. alternate, ovate, fleshy, spurred at the base: eyme bifid, smooth.

4 L. alternate, somewhat ovate, fleshy, spurred at the base: eyme trifid, leafy.

5 L. subternate, somewhat eylind. obtuse, Heshy, sessile, spreading, spurred at the base, inbrieated in 6-rows: eyme trifid, leafy.

6 L. alternate, linear, flattislı: somewhat hairy as well as the pedune: stem ereet.

7 L. oblong, cylind. obtuse, spreading, smooth : pan. mueh branehed. 
CLASS X. DECANDRIA.

ORDER IV. PENTAGYNA.

$=1$

LINNEAN NAMES.

ENGIISII NAMES.

SEDUNI.

8 reflexum ....

STONFCROP.

9 glaucum .... yellow.................

10 rupestre rock

11 Forsterianum.

Forster's.

\section{官敌害}

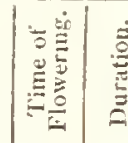

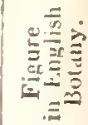

Walls.. yellow 7 P

695

Bar. san. yellow

7,8

I.

2477

Rocks! yellow

7 I'.

170

Wel.roc. yellow

7 I

1802

OXALIST.

\section{WOOD SORREL}

1 Acetosella ...

2 corniculata.

AGROSTEMIA.

1 Githago .... corn

COCKLE.

\section{YCHNIS.}

1 Flos-Cuculi.. ragged robin .... Moi.mea. rosc..

2 Viscaria $\ldots . .$.

German catclifiy. .

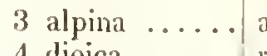

\section{CAMPION.}

4 dioica ..... red or whitc .... Mo.sh. pl. redorw

\section{Corn fi. purple 6,7}

A.

741

1726

P. 762

\section{CERASTIUM. MOUSE-EAR CHCKWEFI)}

1 vulgatum.... broad leaved .... San. pl.

2 viscosum .... narrow leaved.... Pasture

3 semidccandrum

4 tetrandrum ... t

II alls..

white

4,5

A.

789

5 arvense...... field Sall. sh.

white

$5-9$

P. 790

1,5 A. 16:30)

5,6 A. * * 166

(5) a...

Fields .. white

$5-8$

P.

6 alpinum alpinc

W. alps whitc

6,7

'?.

472

7 latifolium .... broad rough .... W. alp whitc 


\section{SHII)UN.}

L. subulate, scattcred, spurred at the base; lowermost rceurved: Fl. somewhat cymose: cal. segm. ovate.

L. glancous, subulate, scattcred, spurred at the base; thosc of the branehes filiform: Fl. eymose: cal. segm. lanccolate.

L. glaucous, subulate, crect, 5-rowed, crowded, spurred at the base: Fl. somewhat cymose: cal. segm. elliptical.

L. semieylind, spreading, clustered in many rows, spurred at the base: Fl. cymose: cal. short, rounded.

\section{OXALIS. Oralider. De C.}

Seape 1-flow: L. ternatc, obcordate, hairy: root sealy-jointed: stam. simple.

Stem branched, diffuse : pedunc. umbelliferous: petioles with stip. at the base: stam. monadelphous.

\section{AGROSTEMMA Caryophylled. IUss.}

Iairy : cal. surpassing the corolla : petals undivided, without teeth.

\section{LYCHNIS. Caryophyller. Juss.}

1 Pet. 4-eleft: fruit roundish, 1-eelled: stem rough with deflexed bristles.

2 Pet. slightly-cloven: fruit 5-celled: L. ciliate at the base.

3 Pet. bifill : Fl. eorymbose: Fr. 5-celled: L. linear-laneeolate.

It Fl. dicecious: pet. eloven, crowned with 4 tceth: fruit I-eelled.

\section{CERASTIUM. Caryophyller. Juss.}

I1 Hairy, viscid, tufted: L. ovate: pet. equal to the cal: Fl. longer than their peduncles.

2 Hairy, viscid, diflusc: L. lanceolate-oblong.

3 Hairy, viseid: Fl. pentandrous : pet. notched.

4 Hairy, somewhat viscid: Fl. 4-cleft, tctrandrous: pet. obcordate, shorter than the calyx. Sagina cerastoides, fing. Bot.

5 L. linear-lanceolate, obtuse, fringed at the base: pet. twice as long as the calyx.

16 L. elliptical, naked or hairy: pan. dichotomous, few-flow. furnished with bractex: caps. oblong, recurved.

7 L.elliptical, rough : pedunc. term. simple, mostly solitary: caps. ovate.

:8 L. cordate, sessilc: pedunc. latcral, solitary, fruit drooping: caps. ovate, mouth 5-toothed: pet, bifid. 


\begin{tabular}{|c|c|c|c|c|c|c|}
\hline \multicolumn{2}{|c|}{ CLASS X. DECANDRIA. } & \multicolumn{5}{|c|}{ ORDER IV. PENT MGYNIA. } \\
\hline LINNEAN NAMES. & ENGLISII NAMES. & 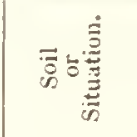 & 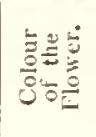 & 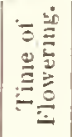 & $\begin{array}{l}\dot{\vec{E}} \\
\stackrel{\vec{E}}{E} \\
\dot{a}\end{array}$ & 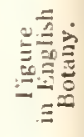 \\
\hline 1 arvensis .... & rough seeded corn & San. fi. & white & 6,7 & A. & 1535 \\
\hline 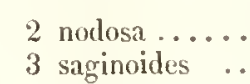 & $\begin{array}{l}\text { knotted ........ } \\
\text { smooth awl shaped }\end{array}$ & $\begin{array}{l}\text { San. hea. } \\
\text { Sc. alps }\end{array}$ & $\begin{array}{l}\text { white } \\
\text { white }\end{array}$ & $\begin{array}{c}7,8 \\
6\end{array}$ & P. & $\begin{array}{r}694 \\
2105\end{array}$ \\
\hline 4 subulata ... & ciliated awl shaped & San. hea. & white & 7,8 & P. & 1082 \\
\hline
\end{tabular}


SPLCIFIC CHARACTER.

245. SPERGULA. Caryophyllea. Juss.

L. verticillate: pedunc. of the fruit, reflexed: seeds kidney-shaped, rough.

L. opposite, subulate, snooth; uppermost tufted: cal. nerveless.

L. opposite, subulate, awnless, naked: pedunc. solitary, very long, smooth.

L. opposite, subulate, awned, fringed: pedunc. solitary, very long, somewhat laairy. 



\section{CLASS XI.}

\section{DO D ECA N D R A.}

\section{ORDER I. MIONOGYNIA.}

Asanum. Cor. 0. Cal.3-cleft, superior. Caps.6-celled.

Lyтним. Pet.6. Cal.12-cleft, inferior.

ORDER II. DIG YNIA.

Acrimonia. Pet.5, inserted in the calyx. Seeds 2 , in the bottom of the calyx.

ORDER III. TRIGYNIA.

Reseda. Pet. many-cleft. Caps, 1-celled, gaping.

(TETRAGYNIA.)

Tormentilia 1.

ORDER IV. DODECAGYNIA.

Sempervivur. Pet.12. Cal.12-parted. Caps. 12. 
CLASS XI. DODECANDRIA. ORDER I. MONOGYNIA.

\begin{tabular}{|c|c|c|c|c|c|}
\hline LINNEAN NAMES. & ENGLISH NAMES. & 官施咅 & 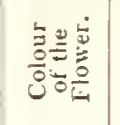 & 兘 & 递 \\
\hline $\begin{array}{l}\text { ASARUM. } \\
1 \text { europieum .. }\end{array}$ & $\begin{array}{l}\text { ASARABACCA. } \\
\text { common ....... }\end{array}$ & Nor.wds! & purple & 5 & $I^{\prime}$ \\
\hline $\begin{array}{l}\text { LYTIIRUM. } \\
1 \text { Sulicaria .... } \\
2 \text { hyssopifolium }\end{array}$ & $\begin{array}{l}\text { LYTHRUM. } \\
\text { purple ........ } \\
\text { hyssop leaved . }\end{array}$ & $\begin{array}{l}\text { Riv. ban. } \\
\text { Wat.pl.! }\end{array}$ & le & $\begin{array}{c}7,8 \\
8\end{array}$ & \\
\hline
\end{tabular}

AGRIMONIA. AGRIMONY.

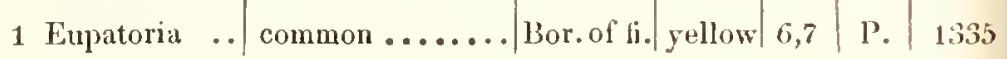

ORDER II. TRIGYNIA.

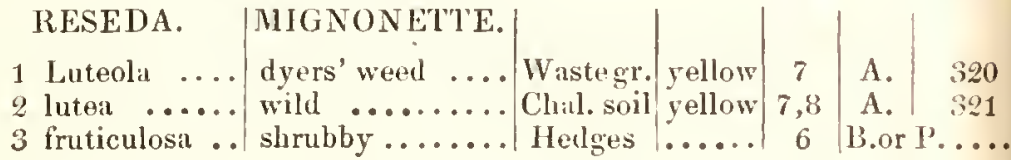

ORDER IV. DODECAGYNIA.

$\begin{array}{r}\text { SEMPERVIV UM. HOUSE-LEEK. } \\ \text { \& tectorum .... } \\ \hline\end{array}$ 


\section{CLASS XI. DODECANDRIA. ORDER I. MONOGYNIA.}

SPECIFIC CHARACTER.

246. ASARUM. Aristolochice. Juss.

L. 2, reniform, obtuse.

247. LithruU. Salicaria. Juss.

1 L. opposite, cordite-lanceolate: Fl. spiked, dodecandrous.

g L. alternate, linear-lanceolate: Fl. axillary, solitary, hexandrous.

ORDER II. DIGYNIA.

248. AGRIMONIA. Rosacer. Juss.

11 Stem leaves pinnate: terminating leafl. petioled: fruit hispid.

\section{ORDER III. TRIGYNIA.}

249. RESEDA. Resedacer? DE C.

1 L. lanceolate, entire, flat : calyx 4-cleft.

2 L. all trifid; lowermost pinnatifid : calyx 6-cleft.

: L. pinnate, waved, glaucous : calyx 5-cleft : petals 5, nearly equal, trifid.

ORDER IV. DODECAGYNIA.

250. SEMIPRVIVUM. Crassulacea. DE C.

1 L. fringed: offsets spreading : edges of the pet. Jairy, entire. 



\section{CLASS XII.}

\section{COSA N D R I A.}

\section{ORDER I. MONOGYNIA.}

1 Pruves. Cal.inferior, 5-cleft. Pet.5. Drupa with anentirenucleus. Mespilus 1 .

\section{ORDER II. PENTAGYNIA.}

12 Mespilus. Cal. superior, 5-cleft. Pet. 5. Apple with 2-5 bony single-valved capsules. Seeds 2 .

.3 Pynus. Cal. superior, 5-cleft. Pet. 5. Apple with 2-5 membranous 2 -valved capsules. Seeds 2.

-4 Spirea. Cal.inferior, 5-cleft. Pet.5. Caps.2-valved, many-seeded.

ORDER III. POLYGYNIA.

i5 RosA. Cal.5-cleft. Recept. fleshy, urn-shaped, contracted at the mouth, at length berried, many-seeded. Pet. 5 .

j6 Runus. Cal. 5-cleft. Berry superior, compound, granules 1-seeded. Pet. 5.

59 Tormextilla. Cal. 8-cleft. Pet. 4. Seeds naked, awnless. Recept. dry, obsolete.

51 Drias. Cal.8-10-cleft. Pet. 5 or 8. Seeds downy, with a long feathery awn.

57 FraGarta. Cal. 10-cleft. Seeds naked, smooth, fixed to a berrylike, deciduous receptacle.

58 Potextrlla. Cal. 10-cleft. Seeds naked, wrinkled, awnless. Recept. dry, obsolete.

:60 Geum. Cal. 10-cleft. Seeds with a jointed awn. Recept. columnar. 162 Comarum. Cal. 10-cleft. Seeds naked, smooth; fixed upon an ovate, spongy, downy, permanent receptacle. Spirat 2, 3. 


\begin{tabular}{|c|c|c|c|c|c|c|}
\hline LINNEAN NAMES. & ENGLISH NAMES. & 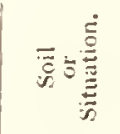 & 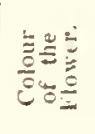 & 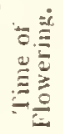 & 咅 & $\begin{array}{r}. \\
\equiv\end{array}$ \\
\hline I Padus ..... & bird .......... & Woods & white & 5 & T. & 1383 \\
\hline 2 Cerasus .... & common .. & IVoods & white & 5 & $\mathrm{~T}$. & 706 \\
\hline 3 domestica & common plum.... & Hedges & white & 5 & T. & 783 \\
\hline 4 insititia.. & bullace .. & Hedges & white & 4 & ' & 8 \\
\hline 5 spinosa.. & sloe...... & Hedges & white & 3,4 & s. & 8 \\
\hline
\end{tabular}

\section{ORDER 1I. PENTAGYNIA.}

\section{MESPILUS. | MEDLAR.}

1 Oxyacantha .. Hawthorn ..... Hedges wh. red 5,6

2 germanica ... common ....... Hedges! white 5 T.

1523

3 Cotoneaster .. dwarf quince-leav. IVel. roc.patered 


\section{SRECIFIC CHARACTER.}

\section{PRUNUS. Rosacer. Juss.}

$1 \mathrm{Fl}$. in pendulous racemes: L. deciduous, with two glands at the base on the under surface.

2 Umbels almost sessile: L. ovate-lanceolate, folded when young, downy beneath.

3 Pedunc. solitary or in pairs: L.lanceolate-ovate, convolute: branches spineless.

4 Pedunc. in pairs: L. lanceolate-ovate, convolute, downy beneath: branches ending in a spine.

5 Pedunc. solitary: L. lanceolate, smooth : branches spinous.

\section{ORDER II. PENTAGYNIA.}

\section{MIESILUS. Pomacer. Juss.}

I Spinous: L. obtusc, mostly trifid, serrated, smooth: styles about 2.

2 Unarmed: L. lanceolate, tomentous beneath: Fl. solitary, nearly sessile, terminal: styles 5 .

3 Unarmcd: I. elliptic-obovate, entire, downy beneath: germen smooth: styles $3-4$.

\section{PYRUS. Pomarea. Juss.}

l L. ovate, serrated: peduncles corymbose.

2 L. ovate-oblong, serrated: Umbèls simple, sessile.

3 L. somewhat corlate, serrated, 7-lobed, lower lobes divaricating: Fl. corymbose. Cratågus, Eng. Bot.

4 L. pinnate, downy beneath: leaf. serrated towards the point: Fl. panicled: Fr. obovate.

5 L. pinnate, sinooth: Fl. corymbose: styles about 3: Fr. globular. Simbus. Eng. Bot.

6 L. pinnatifid, or semi-pinnate, downy beneath: Fl. corymbose: styles about 3.

7 L. ovate, cut, serrated, streaked, tomentous beneath: Fl. corymbose: styles 2 to 4 .

\section{SPIRAA. Rosacer. Juss.}

I L. lanceolate, obtuse, serrated, smooth: raccmes term. compound.

2 L. interruptedly pinnate; lcafl. uniform, serrated, smooth: stem herbaccous: Fl. cymose, with many styles.

3 L. interruptedly pirmate, tomentous beneath; teminal leaflet largest and lobed: stem herbaceous: Fl. cymose, with inany styles. 
CLASS XII. ICOSANDRIA.

ORDER III. POLYGXNIA.

\begin{tabular}{|c|c|c|c|c|c|c|}
\hline & & & & & & \\
\hline LINNEAN NAMES. & ENGLISH NAMES. & 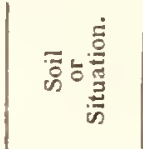 & 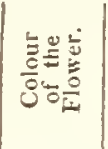 & 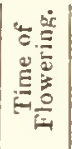 & 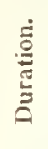 & 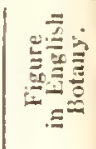 \\
\hline$*$ & & & & & & \\
\hline I einnamomea $\delta$ & cinnamon ..... & Woods! & rose .. & 5 & S. & 2388 \\
\hline 2 rubella..... & red fruited dwarf.. & Sea sh.! & bluslı & 7 & S. & 2521 \\
\hline 3 spinosissima.. & Burnet .... & San. hea. & white & 7 & S. & 187 \\
\hline 4 involuta .... & Dr. Walker's .... & Sc. Isles & blush & 6 & S. & 2068 \\
\hline 5 Doniara ... & Don's ......... & Thiekets & blush & 6 & S. & \\
\hline 6 gracilis..... & sleuder .... & M1. wood! & rose.. & 6,7 & S. & *583 \\
\hline 7 Sabini ... & Sabinian........ & Highl.va. & rose .. & 6,7 & S. & \\
\hline ** & & & & & & \\
\hline 8 villosa $\ldots .$. & apple .... & Nor.mou. & red .. & 6 & S. & $* 2459$ \\
\hline 9 tomentosa... & downy-leaved.... & Hedges & rose . & 6,7 & s. & 990 \\
\hline **** & & & & & & \\
\hline 10 Sherardi ... & Bound-headed .. & lledges & rose.. & 6,7 & s. & \\
\hline 11 rubiginosa & sweet briar ... & Chal.ban. & rose. . & 6,7 & S. & 991 \\
\hline 12 micrantha... & small flow. s. briar & Thickets & pale.. & 6,7 & s. & 2490 \\
\hline
\end{tabular}


LASS XII. ICOSANDLIA. ORDER III. POLYGYNLA.

SPECIFIC CHARACTER.

255. ROSA. Rosaced. Juss.

* Branches bristly. Prickles mostly slender, nearly straight.

Pedunc. bratcterited, smooth: cal. mostly simple: Fr. glohose: bristles decilluous: leafl. oblong, simply serrated, downy, glancous beneath.

Pedunc. and stem bristly : bract. 0 : cal. sinule, bristly : Fr.globose: prickles striight, scattered: leafl. elliptical, smootb, simply serrated.

Pedunc, smooth: bract. 0: cal. simple, smooth: Fr. globose, abrupt, somewhat depressed: prickles straight, uneyual, intermixed with glandular bristles : leafl. roundish, smooth, simply serrated.

Pedunc. bristly : brict. 0: cal. bristly: Fr. globular, bristly : stem bristly and prickly: leafl. elliptical, doubly serrated; their reins hairy beneath: pet. convolute.

Pedunc. globular fr. and simple cal. bristly: bract. 0 : stem bristly and prickly, like the downy petioles: leatt. elliptical, doubly serrated, hairy on both sides : pet. spreading.

Pedunc.mostly in pairs, bristly, often bracteated : brinches,globular fr. and sinple cal.bristly : larger pricklescurved, mostly 2-together: leafl. doubly serrated, hairy on botl sides. R. villosa. Eng. Bot. Pedunc. aggresare, bristly, often bracteated : brauches, globular fr. and pinnate cal. bristly : prickles scattered, straightish: leath. doubly serrated, nearly snooth, with hitiry ribs.

* Branches without bristles. Prickies nearly struight.

Fr. rglobose, depressed, partly bristly: cal. slightly compound. Leah. roundish, bluntish, all over downy. R. mollis. Lug. Bot.

Fr. elliptical, bristly: cal. copiously pimate: pricliles slightly curverl: leatl. ovate, acute, more or less downy.

\section{*** Brunches without bristles. Prickles hooked, compressed.}

\section{Styles distinct.}

Fr. globular, arbrupt, bristly : cal. copiously pinnate. Leafl. elliptical, acute, downy on both sides.

Fr, obovate, bristly towa rus the base : cal. pimate : smaller, straighter prickles interspersed with the larger : le: fl. elliptical, doubly serrated, lairy; clotbed beneath with l'ust-coloured irlands.

Fre elliptical, bristly, contricted at the summit: call. pimute, de(ciduous: stem stritgrgling : leath. ovate, donbly serrated, hairy; matadular beneath. 
CLASS XIl. ICOSANDRIA.

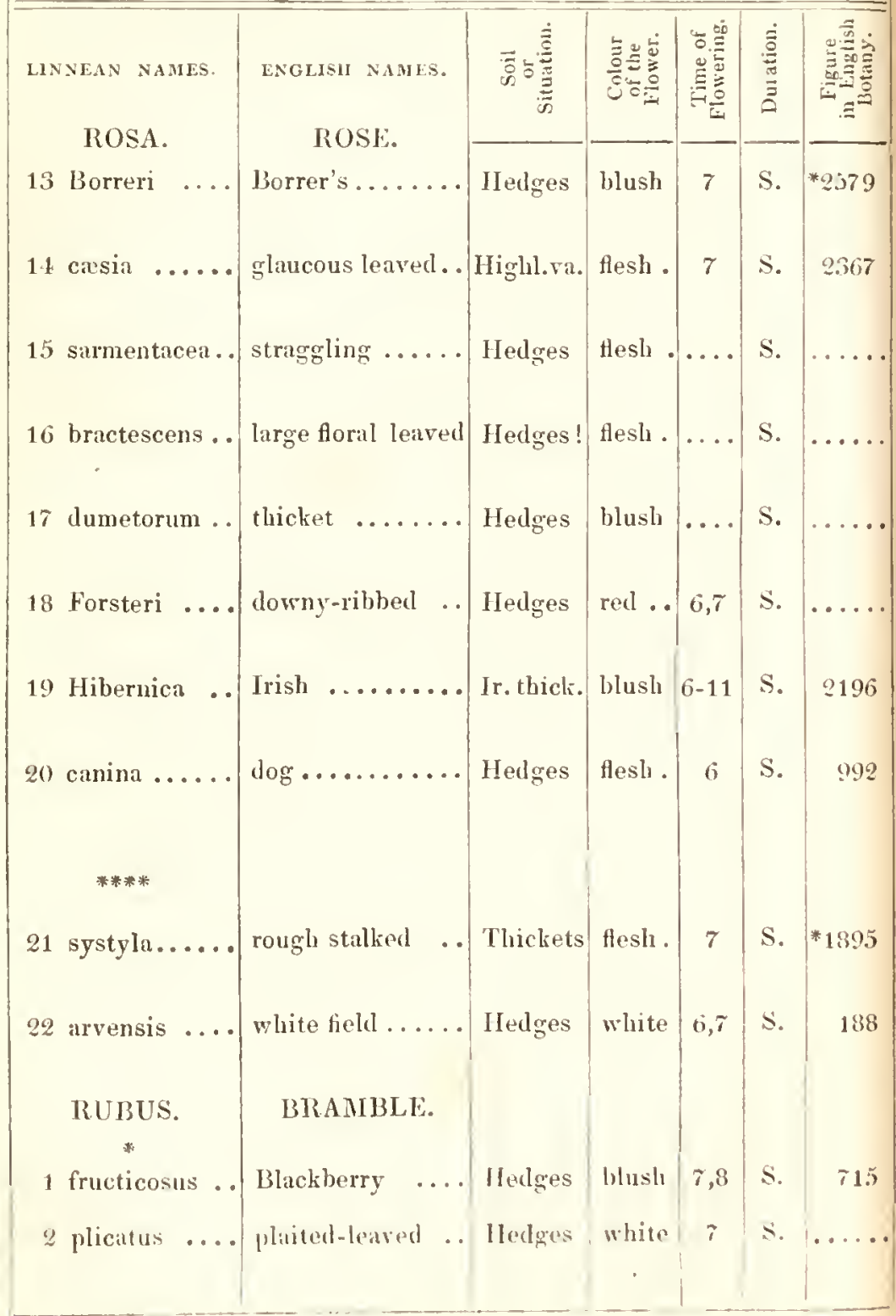




\section{ROSA.}

Fr, elliptical, smooth: pcdunc. aggregate, hairy : cal. copiously, often doubly pinuate, deciduous. Leafl, ovate, doubly-serrated, hairy; without glands. R. dumetorum. Lng. Bot.

Fr. elliptical, smooth: pedunc. smooth, solitary : cal. sparingly pinnate. Leaft. elliptical, doubly serrated, glaucous, hairy beneatls; without glands.

i) Fr. broadly elliptical, naked: pedunc. aggregate, smooth or minutely bristly: cal. strongly pinnate. Leatl. ovatc, doubly serrated, very smooth.

6 Fr.globose, smooth : pcdunc. aggregate, sometimcs hairy : cal.pinnate, with entire lcaflets. Leafl. ovate, almost simply serrated; downy benenth: bract. rising above the fruit.

if Fr. clliptical, smooth, as tall as the bracteas: pedunc. aggregate, slightly hairy: cal. copiously pinnate, cut. Leafl. simply serrated, laairy on both sides.

:8 Fr. elliptical, smooth : pedunc. aggregate, smooth : cal. copiously, somewliat doubly pinnatc. Leaf. sinply serrated, smooth above; ribs bairy beneath.

9 Fr. nearly globular, smooth: pedunc. smooth: cal. pinnate, with entire appendages, permanent. Leafl. elliptical, simply serrited; ribs hairy beneath.

?O Fr. ovate, smooth or ruther bristly: pedunc. aggregate, smooth or rather bristly: cal. pinnate, deciduous. Leatl. simply serrated, pointed, quite smooth.

***** Branches without bristles. Prickles hooked, compressed.

Styles combined.

21 Fr. ovate-oblong, smooth : pedunc. glandular: cal. pinnatc, deciduous. Lcafl. simply serrated: Fl. reccpt. conical. R. collina. Eng. Bot.

22 Fr. globose or elliptical, smooth: pedunc. glandular: cal. pinnate, deciduous. Leatl. simply serrated : Fl. recept. slightly convex.

256. RUBUS. Rosacer. Juss.

* Stem shrubby, angular.

1 Stems furrowed: prickles hooked: L. quinate, downy beneath: leaff. petioled: cal. reflexed, unarmed.

2 Stems angular, ncarly erect : prickles dcflexed : leafl. 3 or 5 , petioled, ovate, plaited ; hoary bencath: cal. rellexed, unarmed. 
CLASS IIJ. ICOSANDRIA.

\begin{tabular}{|c|c|c|c|c|c|c|}
\hline $\begin{array}{l}\text { LINNEAN NAMES. } \\
\text { RUBUS. }\end{array}$ & $\begin{array}{l}\text { WNGLIGII NAMES. } \\
\text { BRAMHLL. }\end{array}$ & 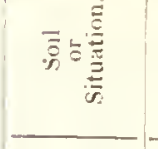 & 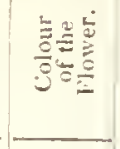 & 至 & 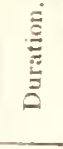 & 要 \\
\hline 3 rliamnifolius.. & buckthorn-leaved. & Hed.Wds & white & 7,8 & S. & 715 \\
\hline 4 leucostachys.. & white-clustered.. & Woods & wh.red & 7,8 & S. & \\
\hline 5 glandulosus. . & glindular $\ldots .$. & Hedges & white & 7,8 & S. & \\
\hline 6 nitidus..... & smooth shining .. & Woods & blush & 7,8 & S. & \\
\hline 7 affinis ...... & ovate hairy. & Hedges & white & 7,8 & s. & \\
\hline 8 suberectus .. & red-fruited ...... & Woods & white & 7,8 & S. & 2572 \\
\hline 9 idreus ..... & raspberry ..... & M. roods & white & 5,7 & s. & $2+42$ \\
\hline 10 corylifolius.. & hazel-leaved ... & Hedges & white & $\gamma$ & s. & 827 \\
\hline 11 crsius .... & dewberry $\ldots$ & Bor of fi. & white & $6, i$ & s. & 890 \\
\hline * & & & & & & \\
\hline 12 saxatilis ... & stone .......... & Nor.wds. & white & 6 & P. & 2933 \\
\hline $\begin{array}{l}13 \text { meticus .... } \\
14 \text { Chandemorus. }\end{array}$ & $\begin{array}{l}\text { dwarf crimson .. } \\
\text { cloud-berry ... }\end{array}$ & $\begin{array}{l}\text { Sto.moor! } \\
\text { Hp. bogs: }\end{array}$ & $\begin{array}{l}\text { rose.. } \\
\text { whiter }\end{array}$ & $\begin{array}{c}5,5 \\
6\end{array}$ & P. & $\begin{array}{r}1.585 \\
716\end{array}$ \\
\hline $\begin{array}{l}\text { FRAGARIA. } \\
1 \text { vesci } \ldots . .\end{array}$ & $\begin{array}{l}\text { STRAWISERRY. } \\
\text { wood ........ }\end{array}$ & Gir. Thick & white & 5,6 & P. & 152.1 \\
\hline E elatior ....... & hauthoy ....... & Woods! & white & 6,9 & P. & $\because 1 ! 7$ \\
\hline
\end{tabular}

\section{ORDER HI. POLYGINIA.}


SPFCIFIC CIIARACTER.

\section{RUBUS.}

Stems furrowed; barren oncs arched: prickles deflexed, straight: L. tcrnate or quinatc: leafl. obovate or cordate, pointed; hoaty beneath: cal. spreading.

+ Stems downy: prickles deflexed: slightly hooked: 1. tcruate or quinatc: leafl. ovate or cordate, pointed, jagged; hoary beneath: calyx reflexed, unarmed, minutely glandular.

i) Branches and footstalks rough with hairs and glandular bristles: prickles deflexed, partly hooked: leafl. 3 or 5 , downy beneath: pan. and cal. prickly and hairy, with copious glandular bristles.

i) Stems spreading, smooth; prickles hooked: leaf. 3 or 5 . hairy beneath: pan. and cal. rather prickly, without bristles.

7 Stems reclining, nearly smooth : prickles hooked: leafl. 1,3 or 5 , ovatecordate, pointed; loosely hairy beneath: cal. hoary, unarmed.

$B$ Stems asccnding, smooth : branches roundish : prickles deflexed: leafl. 3,5 or 7 , ovate-cordate, pointed, minutely hairy beneath: clusters simple, hairy, prickly, on lateral leafy branches: cal. slightly hairy, unarmed.

\section{*** Stems shrubby, round.}

$\exists$ Stems crect, smooth, with downy branches: prickles straight. slender: L. pinnate: leafl. 3 or 5 , ovate, angular, downy beneath : clusters prickly: Fl. pendulous.

כ Stems spreading: barren ones somewhat angular: prickles straight, deflcxed: leafl. 3 or 5 , cordate; finely hairy beneath: pan. and reflexed cal. minutely glandular.

I Stems prostrate, glaucous, prickly, bristly; prickles deflcxcd: leafl. 3. hairy beneatls; lateral ones lobed, externally: cal. embracing the fruit.

\section{*** Stem herbaceous.}

2 Stems ascending, prickly, with prostrate runncrs: L. ternate: pan. few-flow: cal. of the fruit converging, unarmed.

3 Stem unarmed, 1 or 2 -flow. L. ternate: leafl. serrated.

-4 Stem unarmed, 1-flow. L. simple, plaited, lobed: cal. scgm. ovate.

\section{FRAGARIA. Rosacer. Juss.}

1 Hairs of the foot-stalks spreading; those of the pedicles close-presscd, silky.

2 Hairs of the petioles and pedunc. all widely spreading, somewhat deflcxed. 
$=$

LINNEAN NAMES.

POTENTILLA. CINQUEFOIL.

1 fruticosa

shrubby

M.bus.pl. yellow

2 anserina $\ldots . .$. silver weed.<smiles></smiles>

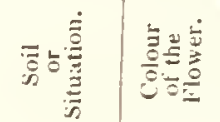

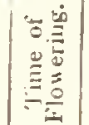

章 告

3 rupestris rock. .

Alp. roc.! white

$\longrightarrow$

$-$

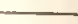

TORMENTILLA TORMENTIL.

I officinalis ... common ........ Bar. pas. yellow

2 reptans .... trailing ....... Mea. pis. yellow

Grav.pa. yellow

5 alpestris

. ho

go

6 verna

spri

7 opaca

saw leaved .

Sc. alps yellow

8 alba

white

W. alps

9 reptans.....

common creeping. Mea. pas. yellow

white

7,8

P.

89

P. *561

***

10 tridentata.... thre

11 Fragariastrum. strawberry-leaved . Bar.pas. white

$6-8$

$\mathrm{P}$.

2449

1384

862

GEUM.

AVENS.

I urbanum ....

common ........

2 rivale wate . Moi. mea. reddish 6,7 P.

P. 863

P. 864

\begin{tabular}{l|l|l|l} 
Woods yellow & $5-8$ & P. & 1400
\end{tabular}

P. 106 
258. POTHN MHLLA. Rosarea. Juss.

* Leaves pinnate.

Leaf. entire, hasy: stem shrubby.

L. interruptedly pinnate, serrated, silky beneatl : stem ereeping: pedune. 1-flow. axillary.

I. lyrate-pinnate, in sevens, fives, and threes: leafl. ovate, serrated, hairy : stem erect.

\section{* Leaves digitate.}

L. quinate, wedge-shaperl, jagged, downy beneath: stem ereet.

Radieal leaves quinate, wedge-shapel, deeply serrated in the upper half; hairy, somewhat membranous; those on the stem ternate: stem almost ereet. P. aurea. Eng. Bot.

Radieal leaves quinate, wedge-shaped, serrated, marked with lines, fringed, somewhat eoriaceous: stem-leaves ternate: stem reclining: eal, segm. partly 3-ribbed.

L. radical of 7 hairy, linear wedge-shaped, serrated leafl: stems deeumbent, with opposite ternate leaves.

L. quinate, silky beneath, eonverging-serrated at the apex: stenis filiform, proeuml : reeeptaele very hairy.

L. quinate, obovate, serrated: stem. creeping: pedune. l-flow: axillary.

\section{*** Lecres ternate.}

I L. ternate, wedge-shaped, smooth above, hairy beneath, trifid at the tips: stem panieled, erect: seeds even.

L. ternate, obovate, serraterl, hairy: stems prostrate: seeds corrugated, hairy at the scar. Fragaria. Eng. Bit.

\section{TORMENTILLA. Rosacer. Juss.}

I Stem rather erect, branehed : L. almost sessile : stipula: cut.

2 Stem prostrate, simple: L. petioled : stipuln undivided.

\section{GEUMl. linsacer. Juss.}

I I. ternate: radical somewhat lyrate: stipula roundish, cut: Fl. erect : awns hooked, naked.

2 Radieal leares lyrate : stipulæ ovate, acute, cut: Fl. nodding: awns twisted, feathered. 
CLASS XII. ICOSANDRIA.

ORDER 1I. POLYGYNIA.

LINNEAN NAMES. ENGLISH NAMES.
CUMARUM. MARSH CINQUEFOLL. 1 palustre .... 


\section{$(58)$}

LASS XII. ICOSANDRIA. ORDER III. POH.YGYNIA.

SPECIFIC CHARACTER.

261. DRYAS. Rosacer. Juss.

Eight-petalled: L. simple, serrated, downy beneath.

262. COMARUM. Rosacea. Juss. 


\title{
CLASS XIII.
}

\author{
POI,YANDRIA.
}

URDER I. MONOGYNIA.

4-petalled.

266 Pabaver. Cal. 2-leaved. Caps. 1-celled, opening witl pores under the crown of the stigma.

264 Chelinonium. Cal. 2-leaved. Silique 1-celled. Seeds crested. 265 Ginacium. Cal. 2-leaved. Siliqne 2 or 3-relled. Seeds dotted. 263 Aстед. Cal. 4-leaved. Berry 1-celled. Seeds flat, placed in a double order.

Cistus 1. occasionally.

* 5-petalled.

270 Cistus. Caps. 3-10-valved, opening at the apes. Cal. 5-leaved, 2-leaflets smaller than the rest.

269 Tiura. C'ups. 5-valved, opening at the base, coriaceous. Cul. 5-parted, deciduous.

\section{Velplinimm 1 .}

******anu-petulled.

267 Nymú, Berry many-celled, with a bark-like coat. Cal. 4-5leaved, larger than the petals. Pet. inserted into the germen. Stig. bearing the Nectury.

268 Nuprar. Berry many-cclled. Cul. 5-6-leaved. 'P'et. inserted with the stamens into the rccept. and bearing honey at their backs.

\section{ORDER 11. PENTALYNIA.}

271 Pifonia. Cal.5-leaved. P'et.5. Stule 0. Caps. many-seeded. 272 Deqphinum. Cal.0. Pet.5; uppermost spurred. Nect.2.cleft. sessile, in the spur.

Reseda 1. Nellebriss. 1, 2.

273 Aconitum. Cal. 0. I'et. 5; uppermost hooded. Nect. 2 recurved, stalked, under the hood.

274 Aruilegra. Cal. 0 . l'et. 5. cqual. Nect. 5, spurred below.

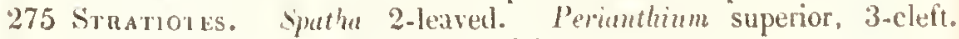
Pet. 3. Berry of 6 or more cells. 
8 Thalictrum. Cal. 0. Pet. 4-5. imbricated. Seeds tailless.

7 Clematis. Cul.0. Pet. 4-8. valvular, or folded in at the edges. Seeds tailed. Recept. capitate.

!6 Anemone. Cul.0. Pet. 5-15, imbricated. Seeds numerous.

2 Helleborus. Cal.0. Pet.5. persisting. Nect.tubular, 2-lipped. Follicles 3-4.

:3 Caltila. Cal.0. Pet.5. Nect. 0. Follicles 5-10.

1 Trollurus. Cal.0. Pet.5-15, deciduous. Nect. Alattened.

.0 Ravurculus. Cal.5-leaved. Pet.5, or more, with a nectariferous claw. Seeds numerous, naked.

9 Anoxis. Cal.5-leaved. Pet.5-15, destitute of nectaries. Seeds numerous, naked. 
CLASS XII. POLYANDRIA. ORDER I. MONOGYNHA.

LINNEAN NAMES. ENGLISH NAMES.

1 spicata ...... common .......

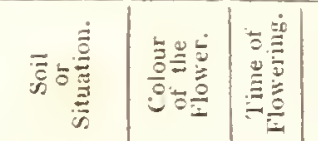

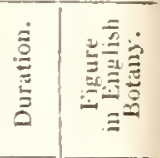

CHELIDONIUM CELANDINE.

1 majus ..... common .......

M. wo.! white 5,6 P'.

918

GLAUCIUM. HORN-POPPY.

I luteum ...... yellow.

San. sh. yellow 7,8

B.

P.

1581

2 phœniceums. San. fi.

3 violaceum....

scarlet.

violet

\section{Chal. fi.! violet 5,6}

A. 1433

A. ${ }^{*} 201$

PAPAVER.

\section{POPPY.}

1 hybridum .... round rough-lieaded Chal, fi.! scarlet

A.

2 Argemore .. long rough-headed Corn fi.

scarlet $6,7 \quad$ A.

643 **

3 dubium. . Iong smooth-headed San. fi.

scarlet 6,7

A.

644

4 Rhoeas common red

Corn fi.

scarlet

6,7

A.

645

5 somniferum.

white san. fens white

A.

2145

6 cambricum .. yellow. Mo.sh.pl. yellow

\section{NYMPII EA.}

WATER-LILY.

1 alba

\section{NUPHAR.}

1 lutea.

2 pumila white ........ Riv. Ac.

white

\section{YELLOW WATER-LIIY.} common ........ l'ools, sce. yellow 
'LASS XIII. POITANDRIA. ORDLRR 1. MONOGINAA. SPEEIFIC CHARACTER.

263. ACTA A. Ranunculacer spurie. Juss.

Cluster ovate: pet. the length of the stamens.

264. CHELIDONIUM. Papaveracer. Juss.

265. (iLAUCIUMI. Papareracer. Juss.

Stem smooth: L. on the stem wavy: silique eovered with roughish tubereles. Chelidminm Cilancium. Eng. Bot.

Stem hispid: L. on the stem pinnatifid, jagged: silique bristly.

L. bipinatifid, linear, smooth : stem smooth: siliques 3-valved.

Chelidonium Hylnidum. Eng. Bot.

266. PAPAVER. Papaceraceri. Juss.

* Capsules hispid.

Caps. almost globular, furrowed : cal. hairy : stem leafy, many-flow. L. bipinnatifid.

¿Caps. club-shaped, ribbed: cal. hairy : stem Icafy, many-flow. L. bipinnatifid.

* Capsules smooth.

i3 Caps. oblong, angular: stem many-flow. hairy : bristles on the pedune. appressed : L. bipinnatifid.

14 Caps. almost globular: stem many-flow. bristly: all the bristles spreading: L. pinnatifid, jagged : stig. many-rayed.

i) Cal. smooth : L. cmibracing the stem, jagged, glaucous.

.6 Caps. ollong, beaked: stem many-flow. smoothish : L. pinnate, jagged, petioled.

\section{NYMPIHA. Nymphacer. DE C'.}

1 L. cordate, very entire, even beneath : pet. elliptic-oblong: stig. 16-cleft, recurved : root liorizontal.

\section{NUPIIAR. Nymphracer. DE C.}

:1 Cal. 5-leavcd : stig. entirc: pctioles 2-edged : L. heart-shaped, with the lobes approximating. Nymphtare. Eng. Bot.

:2 Cal.5-leaved: stig. toothed: petioles 2-edged: L. heart-shaped, with the lobes rather distant. 
CLASS XI11. POLYANDRIA.

ORDER 1. MONOGYNIA.

LINNEAN NAMES. ENGLISII NAMES. |

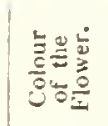

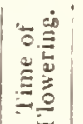

THLIA.

LIME-TRET.

1 europæa

common a.s....

Woods yell-ish 7

T.

610

2 grandifolia .. broad-leaved

Woods

yell-ish 6,7

T.

3 parvifolia.... small-leaved .... Woods yell-ish 8

T.

I 705

CIsTUS.

CISIUS.

1 marifolius.... hoary dwarf ....

Alp.roc.! yellow

5,6

S.

396

2 guttatus .... spotted flowered .. San. pas.! yellow

3 ledifolius .... ledum-leaved .... San. pas.! yellow

6,7

A.

544

4 surrejanus .. dotted leaved .... Chal.hil.! yellow

6,7

A.

2414

5 Helianthemum common dwaif .. Grav.ban yellow

7,8

S. 2207

S Helianthemum com

6 sc. alps yellow 7,8 S.

1321

tomentosus ... downy

7 polifolius.... white mountain

\section{ORDER 11. PENTAGYNLA.}

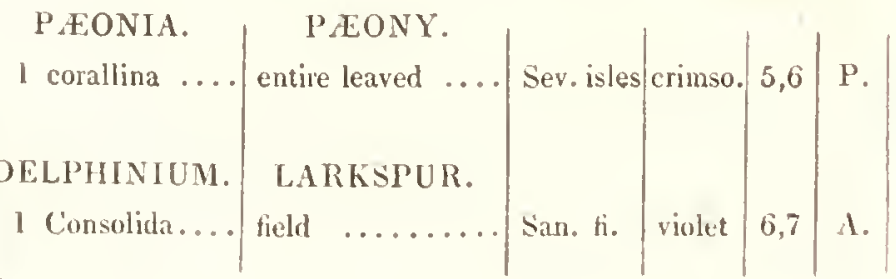




\section{SPECIFIC CHARACTER.}

\section{TILIA. Tiliacer. IUSs.}

Cymes many-flow : nect. 0. L. twice the length of the petioles, cordate; ramification of their veins villous beneath: caps. coriaceous, downy.

: L'mbels 3-How, nect. 0. L. cordate, downy; origin of their veins woolly: branches hairy: woody, downy, turbinate, 5 -angled.

3 Umbels compound, many-flow. Nect. 0. L. smooth above; glaucous beneath, with scattered, as wcll as axillary, hairy blotches: caps. roundish, brittle, nearly smooth.

\section{CISTUS. Cistiner. Juss.}

1 Shrubby: without stip: L.opposite, petioled, oblong, flat, tomentous beneath.

2 Herbaceous: without stip: L. opposite, lanceolate, 3-nerved.

3 Herbaceous: with stip. downy. L. lanceolate: peduncles erect, opposite to the leaves: shorter than the calyx.

4 Shrubby: procumbent, with stip: L. ovate-oblong, hairy, and dotted beneath : pet. lanceulate.

15 Shrubby: procumbent, with fringed stip: L. elliptic-oblong, hoary with hairs beneatlı : caly $\mathrm{x}$-ribs bristly ; its outer leaves lanccolate, fringed.

16 Shrubby: procumbent, with hoary stip: L. elliptic-oblong, snow white, with starry pubescence beneath: cal. loary; ribs hairy : outer leaves obtuse.

7 Shrubby: procumbent, with stip: pubcscence starry: I. oblong, revolute, white and downy beneath: cal. hairy; its outer lcaves fringed.

ORDER II. PENTAGYNHA.

271. PEONIA. Ramunculacer. Juss.

I L. biternate: leafl. ovate, undivided, smooth; seed-vessels downy, recurved.

272. DELPHNNUN. Ranunculacer. Juss.

1 Caps. solitary: nect. 1-leaved: stem subdivided, spreading. 


\section{CLASS XIII. POLYANDRIA. ORDER II. PENTAGYNLA.}

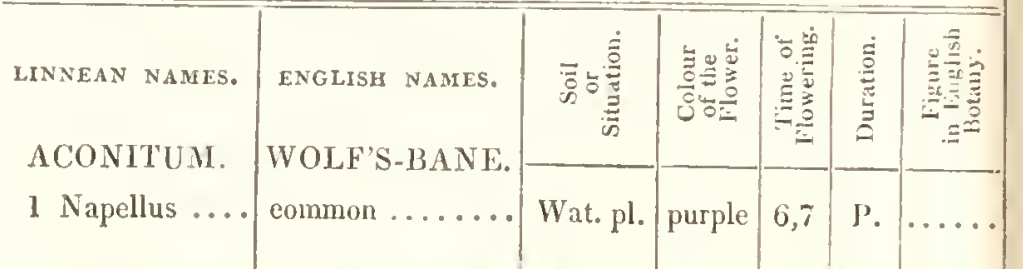

ORDER III. POLYGYNIA.

STRATIOTES. WATER-SOLDIER.

1 vulgaris ....

COLUMBINE.

common ........ Mea.pas. violet

ANEMONE.

ANEMONE.

l Pulsatilla... pasque flower...

2 nemorosa ... woor

Chal. pa. violet

4,5 P.

3 apennina .... blue mountain ..

4 ranunculoides. yellow wood ... -Woods white 4 1'.

355

3 apennina .... CJEMATIS. TRAVELLER'S IOY.

1 Vitalba . common ........ Hedges

THALICTRUM. ILADOIV-RUE.

1 alpinum .... alpine ......... Alp. pas. white

2 minus lesser Chal.pa. pur-ish 6,7

l'. 262

3 inajus ...... greater - M. thick. gre-ish 6,7

4 flavum common Noi.mea. cream P. 
LASS XIII. POLYANDRIA. ORDER II. PENTAGYNIA. STECITIC CHARACTER.

273. NCONITUM. Ranunculaces. Juss.

Yet. upper arched at the back; lateral hairy inside: germ. 3. smooth: L. 5-cleft, cut; segm. linear, furrowed above.

274. MQULLEG1A. Ranunculacer. Juss.

I Nect. incurver, scarcely equal to the petals : stem and leaves smooth: caps. hairy.

275. STRATIOTES. Hydrocharider. Juss.

11 L. ensiform, channclled, with a prominent rib, and sharp marginal prickles.

\section{ORDER III. POLYGYNIA.}

\section{ANEMONE. Ramenculacer. Juss.}

1 Stalk 1-flow: petals 6 , erect: involucrum in many lincar segments: L. bipinnate, cut : seeds tailed.

2 Stalk 1-flow: pet. 6, elliptical: invol. 3-lcaved; leaft. ternate or quinate, loberl, and cut, petioled : seeds tailless.

3 Stalk 1-flow: invol. 3-leaved: leatl. ternate, petioled: secrls tailless: petals lanccolate, numerous.

4 Stalk mostly 2-flow: invol. 3-leaved, almost sessilc, deeply cut: seeds tailless : petals 5 , elliptical.

\section{CLEMATIS. Ramunculaced. Juss.}

1 L. pinnate: leaf. cordate: petioles twining: pan. forked; not longer than the leaves.

278. THALICTRUM. Renunculacer. Juss.

1. Stem very simple, almost naked: cluster simplc, tcrminal.

2 L. bipinnate; leaf. ternatc, hifid, glaucous: Fl. panicled, drooping: stem zigzag: stip. rounded.

3 L. tripinnate; leatl. ternate, lobed, glaucous beneath: branches of the pan. crowded, somewliat umbclled: Fl. drooping: stip. crescentshaped, notcherl.

4 L. bipinnate; leat. trificl: stem furrowed : pan. much branched, conpact: Fl. erect. 
CLASS XIII. POLYANDRIA. ORDER II. POLYGYNIA.

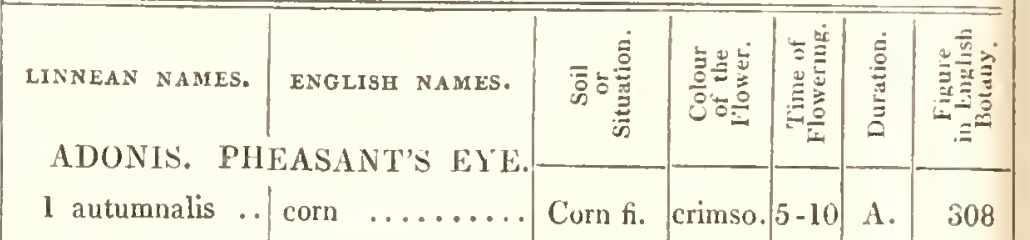

l Flammula .. lesser spear-wort. .

Corn fi. crimso. 5-10 A.

308

2 Lingua...... great spear-wort..

Wat.pl. ycllow

$6-9$

P.

387

3 gramincus

g

Mud. dit. yellow

7

P.

100

4 Ficaria.....

Alp. mea. yellow

P.

2306

pilewort ...

Bush. pl. yellow

P.

584

5 auricomus

wood

Bush. pl. yellow

4,5

P.

624

6 sceleratus Wat, pl. yellow 6-8

A. 681

7 alpestris

Alp. riv.! white

P.

2390

8 bulbosus b

9 hirsutus .... pal

pale hairy

Mlea. pas. yellow

P.

515

10 repens ......

11 acris.....

creeping ........Mea. pas. yellow 6 . upright mearlow .. Mlea. pas. yellow

12 arvensis com......... Corn fi, yellow

13 parviflorus ... s

14 hederaceus .. ivy-leaved Grav. pl. ycllow

15 apuatilis .... whi

white foating....

IVat.pl. white

5,8

P.

2003

TROLLIOS, GLOBE-FLOWFR.

I europatus.... common ........ Mo.sh.pl. yellow $5,6,1^{\prime}$

I. 
LASS XIII. POIYANDRIA. ORDER IJI, POLYGYNIA.

SPECHFIC CHARACTER.

279. $\triangle D O N I S$. Ramuneulacca. Juss.

Petals about 8 , obcordate: fruit ovate: stem branched.

280. RANUNCULUS. Rammonlacete. Juss.

* Leaces simple.

L. ovate-lanceolate, rather obtuse, petioled: stem declining: root fibrous.

2 L. lanceolate, acuminate, nearly sessile, serrated: stem ercct, manyHow : root fibrous.

i) L. linear-lanceolate, many-nerved, sessile : stem erect, few-flow. very smooth: root tuberous.

It L. cordate, angular, petioled, shining: pet. many, elliptic-oblong.

** Leaves lobed, or cut.

5 Radical leaves reniform, 3-parted, crenate: stem leaves digitate, linear: stem many-flow: calyx colourerl.

6 Lower leaves palmate; upper digitate: fruit oblong: stem erect, hollow, much branched: cal. hairy, reflexed.

7 L. smooth, subcordate, 3-parted, lobed: stem mostly I-flow. with one lanceolate entire leaf: cal. smooth, reflexed.

$: 8 \mathrm{Cal}$. rcflexed: pedunc. furrowed: stem ercct, many-flow: L. compound: root bulbous.

9 Cal. reflexed, acuminate: stem erect, many-flow. hairy: 1. tcrnate: root fibrous: seeds tuberculated.

0 Cal. spreading : pcdunc. furrowed : runners creeping: L. compound.

1 Cal, spreading : perdunc. cylind: L. 3-parted-many-cleft; upper ones linear : stem crect, covered with close hairs.

2 Seeds prickly : L. 3-cleft-decompound; seginents lincar : stcm crect, much branched, many-flow.

3 Seeds prickly; prickles hooked: L. simple; segments acutc, hairy: stem diffuse.

4 L. 1eniform-roundish, 3 or 5 lobed, very entire, smooth : stem creeping: seeds wrinkled.

5 L. under-water capillayy; thosc above somewhat peltate, lobed, notched : secds wrinkled.

281. TROLLIUS. Ranunculacea. Juss.

1 Pet. about 15 , converging: nectaries 5-10, the length of the stamina. 


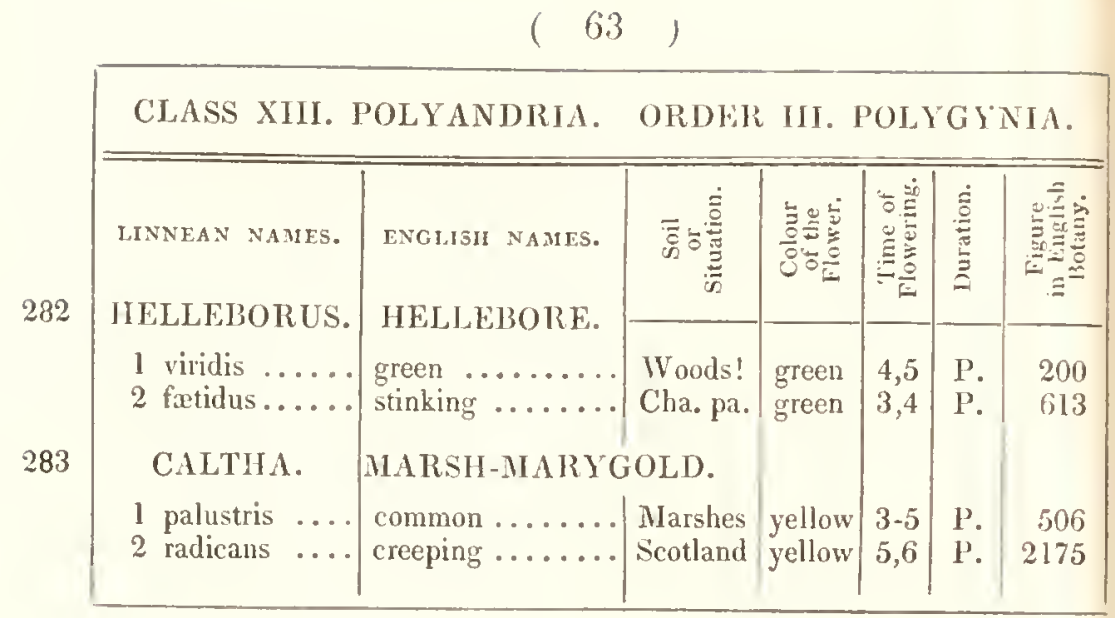


ILASS NIH. POLYANDRIA. ORDER III. POLYGYNIA.

SPECIFIC CHARACTER.

282. HELLEBORUS. Ranunculacer. Juss.

Stem many-flow, leafy: L. digitatc: petals spreading. Stem many-flow. leafy : $L$. pedate: petals converging.

283. Ciltila. Ranunculacee. Juss.

L. cordate: stem erect.

L. triangular, subcordate, sharply crenate : stem crecping. 


\title{
CLASS XIV.
}

\author{
DIDYNAMIA.
}

\section{ORDER I. GIMINOSPERMIA.}

* Calyres mostly 5-cleft.

297 Leowunus. Anthers sprinkled with boney dots. Cor. upper lip shaggy.

289 Gileciona. Auth. eachpair forming a cross. Cur. upper lip cloven. 288 Mехтил. Filuments distant, straighti. Cor. nearly regular, 4-cleft. 285 Teucriun. Cor. upper lip 2-parted, lobes remote.

284 AJuga. Cor. upper lip very small, and shorter than the stam.

293 Betonica. Cor. upper lip flat, ascending, tube cylind. incurved. Stam. as long as the throat.

290 Lamum. Cor. throat toothed on both sides.

291 Galeopsis. Cor. lower lip with two hollow prominences at the base in front.

292 Galeobnolox. Cor. lower lip 3-cleft, segments acute.

294 Sracirs. Cor. lower lip reflexed at the sides. Stam. reflecterl to the sides, after shedding their pollen.

286 Nepres. Cor. lower lip crenate, throat with the margin reflexed.

295 Bariota. Cul. 10-striated. Cor. upper lip archer, shaggy.

296 Marrubus. Cal. 10-striated. ('m. upper lip straight, 2-cleft. 287 Verisas. Cal. 1 tooth truncated. Cor. nearly equal, curved.

$$
\text { ** Calyres 2-lipperl. }
$$

302 Scuterdania. Cal. when in fruit covercd with a lid.

300 'Tnyos. C'al. throat closed with oft hairs. Cor. upper lip flat, notched.

301 Menitris. Cal. wider than the tube of the corolla. Cor. upper lip flat. Anth. crosscd.

298 Cusopodun. Inmolucrnm many-leaved, collecting the caiyres. Cal. many-ribbed.

299 Onganum. Calyxes collected, by imbricated bracteax, into a spicatc, 4 -angular cone : ribless.

303 Prunisia. Filuments all 2 -forked at the apex. 
LASS XII. DIDYNAMIA, ORDER II. ANGIOSPERMIA.

$$
\text { * Calyres 4-cleft. }
$$

Latira. Caps, l-celled. Glaml under the germen.

Bartsia. Caps. 2-celled. Seeds angular.

Rinnsintus. Caps. 2-celled. Seeds compressed Aat, imbricated.

Menapytur. Caps. 2-celled. Seeds 2, gibbous, shining.

Evpirasia. Caps. 2-celled. Seeds striated. Anth. spinous.

$$
\text { * Calyres 5-cleft. }
$$

Limoselta. Caps. impcrfectly 2-celled. Cor. bell-shaped, nearly cqual.

Scropuvlaria. Cups 2 -celled. Cor. reverscd, tube inflated; lip with an intermediate segment within.

Sibthorpla. Caps. 2-celled, with transverse partitions. Cor. nearly wheel-shaped. Stam, each pair approximate. Digitalis. Caps. 2-celled. Cor. bell-shaped, ventricuse beneath : Stam. bent.

Avtrarnives. Caps. 2-celled. Cor. mouth closed by the palate, spurred, or bulging at the base, behind.

Pedicularis. Caps. 2-celled. Seeds mucronate. Cor.personate; helmet compressed.

Lixiea. Berry 3-celled, dry. Cor. bell-shaped. Cal. double; innermost superior.

**** Cal. 2-leaved.

; Oróanche. Cal. leaflets lateral, lobed. A gland under the germen. Caps. 1-celled, with 4 receptacles. 
CLASS XIV. DIDYNAMIA. ORDLR I.GYMNOSPERMIA.

LINEAN NAMES.

EXGLISII NAMES.

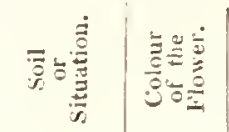

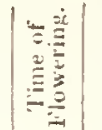

AJUGA.

BUGLE.

1 reptans......

common ........ Mloi.wo. blue

2 pyramidalis .

$$
\text { py }
$$

sic. mount purple

5

P. 489

5,6 P. 1270

3 alpina

a

alpine

4 Chamepitys..

ground pine

.

Mount.! blue

7 P.

477

San. fi.

yellow

4,5

A.

\section{TEUCRIUM. GERMANDER.}

1 Scorodonia ..

wood

Woods yellow

7 I'

1543

2 Scordium .... water Marshes! purple 7,8

3 Chamædrys... wall

Old walls purple

1.

828

286

NEPETA.

1 Cataria.

VERBBNA.

1 officinalis....

\section{CAT-MINT.}

$$
\text { com }
$$

Road si. white

P.

137

.

MENTHA.

1 sylvestris

...

\section{VERVAIN.}

common

Road si.

\section{MINT.}

2 lotundifolia. round-leaved ....

3 viridis ..... spea M

Wat.pl. lilac

8,9

P.

680

Noi. pl. reddish

8,9 J.

446

Marshes purple

8

I'.

2424

4 piperita......

pepper.

Wat.pl. purple

8,9 '?.

687

5 citrata ..... Wat. pl.! red ..

$7-9$

I.

1025

6 hirsita ..... l

Wat.pl. lilac

7 icutifolia frag. sharp leaved. Wat. pl.! lilac

8,9 I'. 


\section{AJUGA. Labiater. Juss.}

Smooth : stem solitary: scyons creeping: cor. lower lip 4-eleft. llairy: whorls elowded into a pyramidal form, many flowered : radical leaves very large, obovatc, erenate, obtuse : eor. upper lip cloven.

Stem simple: L. almost smooth, unequally toothed, uppermost entire: whorls rather remote, many-How: scyons 0.

Stem diftuse, branehed: L. 3-eleft, linear, entire: Fl. axillary, solitary, shorter than the leaves.

\section{TEUCRIUM. Labiater, Juss.}

L. eordate, serrated, petioled: elusters lateral, 1-rowed: stem erect. L. oblong, sessile, toothed-serrated: Fl, axillary, in pairs, peduneled : stem diffuse.

L. somewhat ovate, petioled, deeply erenate: Fl.axillary, peduncled, ternate: stem cylind. lıairy.

\section{NEPETA. Labiater. Juss.}

Fl. spiked: whorls somewhat pedicelled: L. petioled, cordate, dentato-serrated, finely downy.

\section{VERBENA. Terbenacer. Juss.}

Tetrandrous: spikes filiform, panicled: L. deeply jagged: stem mostly solitary.

\section{MENTIIA. Labiater. Juss.}

spikes villous, scarcely interrupted: L. toothed-scrrated, chiefly downy beneath : bractea awl-shaped: cal. hairy.

spikes hirsute, interrupted: L. elliptical, obtuse, wrinkled, crenate, villous, beneath: bractex lanceolate.

spikes interrupted: L. sessile, lanecolate, aeute, naked: bractex setaceous and somewhat liairy, as well as the ealyx teeth.

Spikes obtuse, interrupted below: L. petioled, somewliat ovate, smoothi-h: cal, very smooth at the base.

Spikes capitate, very obtuse: L. petioled, cordate, nakerl on both sides: cal. and pedicels very smooth on every part.

Fl. capitate, or whorled: I. petioled, ovate: cal. hairy: pedieels with liairs pointing backwands.

Fl. wholler : 1. orate-lanceolate, aeute at each $\mathrm{cnd}$ : cal, hairy on every part: hairs of the pedicels recurved. 
CLASS XIV. DIDYNAMA. ORDER I. GYMNOSPERMA.

289
MINT.

tall red

8 rubra

9 gentilis.

10 gracilis......

11 arvensis $\ldots$. corn

12 agrestis......

13 Pulegium.... rugged field

penny-royal

GROUND-IVY.

1 hederacea... common DEAD-NETTLE.

LANIUM.

1 album white

Waste gr. white

5-9

Banks!

2 maculatum .. spotted

3 purpureum .. red

4 incisum

5 amplexicaule. cut-leaved Waste gr. purple San. fi. rose. . San. fi. rose. . henbit GALEOPSIS.

I Ladanum... .

2 villosa .......

3 Tetrahit HEMP-NETTLE. red

Chal. fi. rose. . San. fi.! yellow 7,8

A. 2353 Corn fi. white

4 versicolor .... large flowered.... San. fi. yellow

A. 207 667 (BALKOBI)OLON WEASEL.SNOUT.

I huleum ..... yellow ......... Mo.sh.pl. yollow 
SPECIFIC CIIAUACTEH.

\section{MENTIIA.}

Nl. whorled: L., ovatc: stem erect, zigzag: pedicels and calyx very smooth: teeth hairy.

Fl. whorled: 1. ovate: stcm much branched, spreading : cal. at the base, and pedicels, smootl.

Fl. whorled: L. lanceolate, nearly sessile: stem much branched, erect: cal. at the base, and pedicels, very smooth. M. Centilis, f.l. .

Fl. whorled: L. ovate: stem much branched: cal. bell-shaped, hairy on every part: hairs horizontally spreading.

Fl. whorled: L. somewhat cordate, deeply serrated, rugose: stem erect: cal. hairs horizontal.

Fl. whorled: L. ovate: stem prostrate: pcdicels and calyx downy on every part: teeth fringed.

\section{GLECHOMA. Labiater. Juss.}

L. kidney-shaped, crenate.

\section{LAMIUM. Labiateä. Juss.}

L. cordate, pointed, petioled: whorls 20-flow: cal. tube shorier than its teeth.

L.. cordate, pointed, deeply serrated: whorls 10-How: cal. tube curved, as long as its teeth.

L. cordate, blunt, pctioled; uppermost crowded: cor. tube within bearded at the base.

L. cordate, dilated, petioled, irregularly cut; uppermost crowded: cor. tube internally naked.

Floral leaves sessile, enbracing the stem, blunt.

\section{GALEOPSIS. Labiater. Juss.}

Stem-internodes equal: L. lanceolate, subserrated, lairy ; helmet of the cor. indistiuctly crenate.

Stem-internodes equal : L. ovate-lanceolate, serrated, villous : lielmet of the cor. deeply notched.

Stem hispid: internodes thicker above: cor. twice as long as the calyx : lielmet straightish.

Stem hispid: internodes thicker above: cor. thricc as loug as the ealy $x$ : helmet inflated.

292. GALEOBDOLON. Labiater. Juss. 
CLASS XIV. DIDYNAMIA. ORIER I. GYMNOSPERMIA.

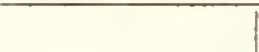

LINNEAN NAMES

(

EMGLISH NAMES.

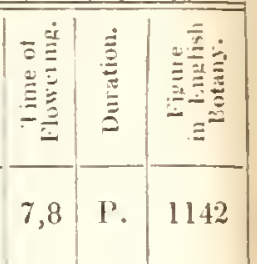

1 officinalis....

BETONY.

STACHYS.

wood...$\cdots \cdot$.

Woods

WOUNDWOR'T.

1 sylvatica ....

hedge .........

2 ambigua $\ldots .$.

4 germanica ...

295

5 arvensis

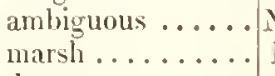

downy............

corn

Hedges blood 7.8

P. 416

P. 2089

P. 1675

Ho. mea. purple 8

Chal. fi. purple 7

$I^{\prime}$.

829

Corn fi. purple 7,8

A. 1154

BALLOTA.

BLACK-HOREHOUND.

1 nigra....

stimking ........ Hedges

\begin{tabular}{l|l|l} 
purple & 7,8 & I'.
\end{tabular}

46

296

MARRUBIUM. WIITE-IIOREHUUND.

1 vulgare......

297

LEONURUS. MOTHERIYORT

1 Cardiaca ....

comnon ....... Grav, ba. white

$7,8 \mid T^{\prime}$.

286

298 CLINOPODIUM

BASIL.

I vulgare.

wild

Grav. ba. rose.

8 P.

1401

299

ORIGANUN.

I vulgare......

MARJORAM.

300

\section{THYMUS.}

1 Serpyllum ..

2 Acinos..... common .........

Chal. wo. rose.. 7,8

P.

THYME.
3 Calamintha .. common calamint. Hor. of fi. violet
4 Nepeta..... lesser calamint .. Chal. hill blue

Heaths

wild $\ldots \ldots \ldots$

Cult. fi. violet 7,8

1514

basil

$7,8 \quad$

411

8 '.

14 I4 
993. HBTONICA. Labiater. Jiss.

"11 Spike interrupted : middle segment of the lower lip of the corolla notched.

99.1. STACHIS. Labiater. Juss.

11 Whorls 6-flow : L. cordate, petioled : stem solid.

2 Whorls 6-flow: L.oblong, petioled, cordate at the base : stem hollow. 3 Whorls 6-10-How: I. linear-lanceolate, half embracing the stem.

4 Whorls many-flow: L. crenate, silky, woolly beneath: stem woolly. 5 Whorls 6-flow: stem feeble: L. cordate, blunt, crenate, somewhat hairy.

\subsection{BAILLTA. Labiater. Juss.}

1 1 L. ovite, undivided, serrated: cal. dilated above, somewhat truncated ; teeth spreading.

996. MARRUBIUM. Labiater. Juss.

1 Calyx-teeth 10, setaceous, hooked.

297. I.EONURUS, Labiatere. IUss.

1 Cpper leaves lanceolate, S-lobed or entire.

\section{CIINOPODIUM. Labiater, Juss.}

1 Whorls hairy : bract. setaceous: pedicels branched : L. indistinctly serruted.

\section{ORIGANUM. Labiater. Jiss.}

1 Splies roundish, panicled, clustered, smooth : invol. ovite, longer than the calyx : cal, throat hairy.

\section{TIIYMlis. Labialea. Juss.}

1 Fl.capitate: stems decumb: L. flat, ovite, blunt, fringed at the base.

¿Whorls f-flow: pedunce simple: stem ascending, branched: L. acute, serrated: calyx ribbous.

3 Pedunc. axillary, many-flow, forked : L.ovate, blunt, slightly serrated, hairy: stem hairy: hairs in the moutl of the calyx, not prominent.

1 P'edunc. axillay, many-flow. forked-corymbose : L. ovate, blunt, serrited, smoothish: stem sonewhit hairy: hairs in tha mouth of the calyx promiunt. 
CLASS MU. DHDYNAHIA. ORDER I. GYMNOSPLRMHA.

MELITTIS. WANTARD-ISLM.

SCUTEI.ARRA. SKITIL-CAP'.

1 galericulati. . common ........ Wit. pl. blue

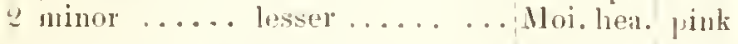

\section{PRUNELLA. SELF-HEAl。}

1 vulgaris ... common....... Merr pis, violet 7.8 P. 961

\section{ORDER II. ANGIOSPERMIA.}

\section{BARTSIA. BARTSIA.}

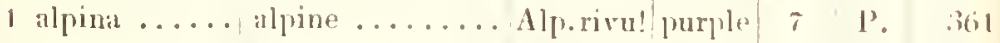
2 viscosa $\ldots$ yellow viscid ... Marshes! yollow $7,8 \quad 1$. 1015

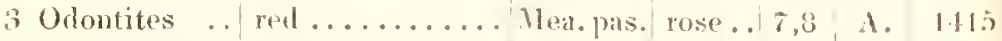

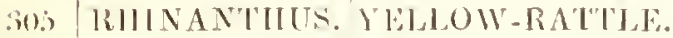

1 Cristi-galli. common....... Mes, pals. vellow $6 \mid$ A. 657

2 major ..... liage bushy .... Corn fi. yellow

\section{EUPHRASIA. HYL-BRIGIIT.}

1 officinalis.... common ...... l'astures White 7.9 A. 1110

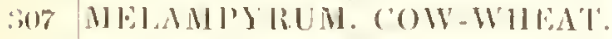

1 cristatum.... rested ........ ('orn fi.! yellow t

"arvense ... purple ........ Corn li. yellow

:3 pratense ... common...... Woots yedlow 7,8

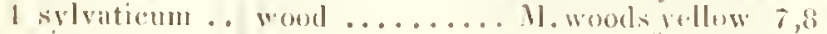

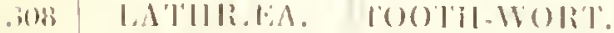

1 Sifnamirial ... wimiter. 


\section{DLASS XIV. DIIYNAMIIA. ORDER I. GYMNOSPLIRMIA.}

SULCIHIC CHARACTIR.

301. MILLl'l"lis. Labiated. Jiss.

1 Calyx 3 -lobed.

2 Calyx t-lobed.

30\%. SCUTLILARIA. Lubiater. Juss.

1 L. cordate-lanceolate, crenate, wrinkled : Fl, axillary.

2 L. cordate-ovate, entire: Fl, axilliny.

30:3. H'RlTNELLA. Labiater. Juss.

iI L. all ovate-oblong, petioled.

\section{ORDER II. ANGIOSPERMIA.}

304. BAR'TIA. Scrophularined. Juss.

1 L. opposite, cordall-ovate, bluntly serrated: stem square; root creeping.

2 1. serrated; upper alternate : Fl. lateral, distant: stem round : root fibrous.

3 J. linceolite, serrated; npper alternate: FJ. racenose, l-rowerl: stem squar', branched; root fibrous.

305. RHINANTIlUS. Scrophulariner. Juss.

1 Upler lip of the cor. arched : cal. smooth: I. lanceolate, serrated. 2 L. linear-lanceolite, upper ones especiatly icuminate: fl. in crowderl spilies: cal. gliblurous; style a little exserterl: seeds wi-ly a nurrow nembranous border.

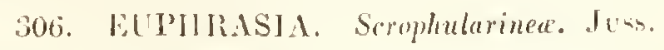

1 f. nvale, stroaked, finely toothed.

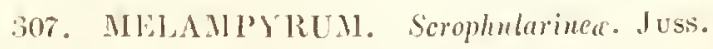

1 silikes s-angular : bract. corlate, imbricaterl, comprict, tootlred.

2 Spikes conical, lax: bract. lincenolite, pinnititid : cal. teeth longer thin thes tube: core closind.

3 Fl.Iiteral, 1-rowed : L. in distant pairs: cor. cIosed : lip extended. 4 Fl. lateral, 1 -rowed : L. in distant pairs: cor. open: lip deflexed.

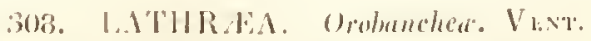

1 Stem very sinple: lil. pendulous; lowre lip tribl. 
CLASS NIV. DIDYNAMIA. ORDER 11. ANGIOSPERMIA.

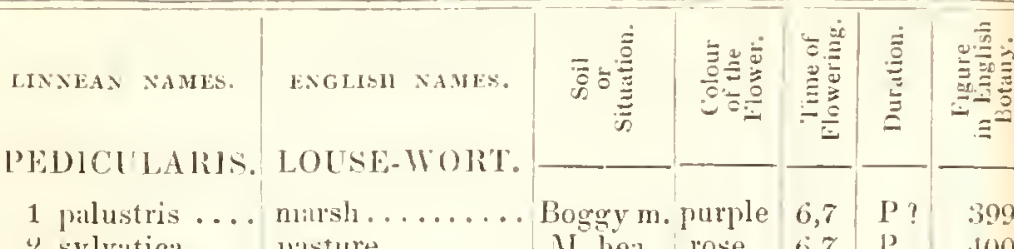

310 ANTIRRHINUM. TOAD-FIAX.

\begin{tabular}{|c|c|c|c|c|c|c|}
\hline * & & & & & & \\
\hline 1 Cymbalarias & iry-leaved..... & Old walls & violet & $5-11$ & $l^{\prime}$. & 502 \\
\hline a spuria ..... & round-leared.... & Corn ti.! & yellow & $7-9$ & A. & 691 \\
\hline 3 Elatine ... & sharp-pointed... & Corn fi. & yellow & $7-9$ & A. & 692 \\
\hline * * & & & & & & \\
\hline . repens..... & creeping ...... & Chal.hil.! & blue & $7-9$ & $1^{\prime}$. & 1253 \\
\hline 5 Linaria & common.... & lledges & yellow & 6,7 & $P$. & $\left\{\begin{array}{l}658 \\
\beta 260\end{array}\right.$ \\
\hline 6 minus.... & least.......... & Sill. fi. & violet & $6-8$ & A. & 9014 \\
\hline 7 majus $\$ . . .$. & great......... & Old walls & rose". & 7,8 & $P$. & 199 \\
\hline 8 Orontium & lesser.......... & San. fi. & flesh. & 7,8 & A. & 1155 \\
\hline CROI'HULARI & 1. FIGWOR'. & & & & & \\
\hline 1 nodosa $\ldots$ & knotty-rooted ... & IYoorls & blood & 7 & P. & 1344 \\
\hline ¿ayuatici .... & water.......... & Wat. pl. & blood & 7 & l'. & 8.4 \\
\hline 3 Scorodonia .. & balm-leaved .... & Sou.riv! ! & $l^{\text {hur }}$ & 7,8 & P. & 2209 \\
\hline 4 vernalis ... & yellow...... & Sh.pl.! & yllow & 1,5 & & 567 \\
\hline DIGITALLS. & FOX-GLOSE. & & & & & \\
\hline 1 purpureal.... & purple $\ldots$. & Hed. ban. & purplo & 6,7 & $\mathbb{P}^{2}$. & $1: 97$ \\
\hline I.1NN.T. & $1.1 N N A: \Lambda$ & & & & & \\
\hline 1 borealis.... & two-Howered. & D) l.s.wds! & flesh. & 5,6 & I'. & $1: 3 i$ \\
\hline Slli'lloliph. & MONLEWOR'T' & & & & & \\
\hline I ruroplas: $\ldots$ & rornish $\ldots$. & '. p'. & yellow & 7,6 & 1. & (it!? \\
\hline
\end{tabular}

SI1 SCROPHULARIA. FIGWORT.

"sylvatica ... palsture........ II. liea.

P. 400

1 Cymbalarials. iry-leaved...... Old walls violet 5-11

502

\& spuria ...... round-leaved...

591

692 
309. PEIICULARIS. Scrophulariner. Juss.

I stem solitary, branched: cal. ovate, hairy, ribbed, in 2 notched lobes.

"S Stems many, simple, spreading: cal. oblong, angular, smooth, in 5 notched segmonts.

310. ANT1RRIINUM. Scrophularinere. Juss.

* Lences diluted: stems procumbent.

11 L. cordatc, 5-lobed, alternate, smooth.

2 L. ovatc, downy, alternate.

3 L. hastate, alternate; lowermost ovate, opposite.

$$
\text { * Jences narruuer: stems erect. }
$$

4 L. linear, glaucous, whorled or scattered: stem panicled: cal. smooth, the length of the spur.

5 L. linear-lanceolate, crowder: stem spiked: cal. smooth, shorter than the spur.

Var. $\beta$. Fl. regular, pentandrous. with 5-spurs. Peloria.

6 L. mostly alternate, lanceolatc, obtuse, pubescent: stem much branched, spreading: cal. longer than the spur.

$7 \mathrm{Fl}$. spiked: cal. obtusc, villous: cor. with a rounded prominence at the base.

8 Fl. loosely spiked: cal. digitatc, longer than the corolla.

\section{SCROPIIULARIA. Serophulariner. Juss.}

1 L. cordate, acute, 3-nerved at the base: stem with acute angles.

2 L. cordate, petioled, dccurrent, obtuse: stem winged.

3 L. corlate, doubly-seniated, tomentous beneath : racemc leafy.

4 L. cordate, doubly-serrated, pubescent: pedune. axillary, solitary, dichotomous, leafy: cor. without an interior lobe.

312. D]GITALIS. Scrophularined Juss.

1 Cal. leafl. ovatc, acute: cor. obtuse, upper lip cntire: L. pubescent.

313. LINNAA. Caprifoliarer. Juss.

1

3H. SIlsTHORPIA. Scrophuleriner. Juss.

1 L. kidney-shaped, somcwhat peltate, crenatc. 


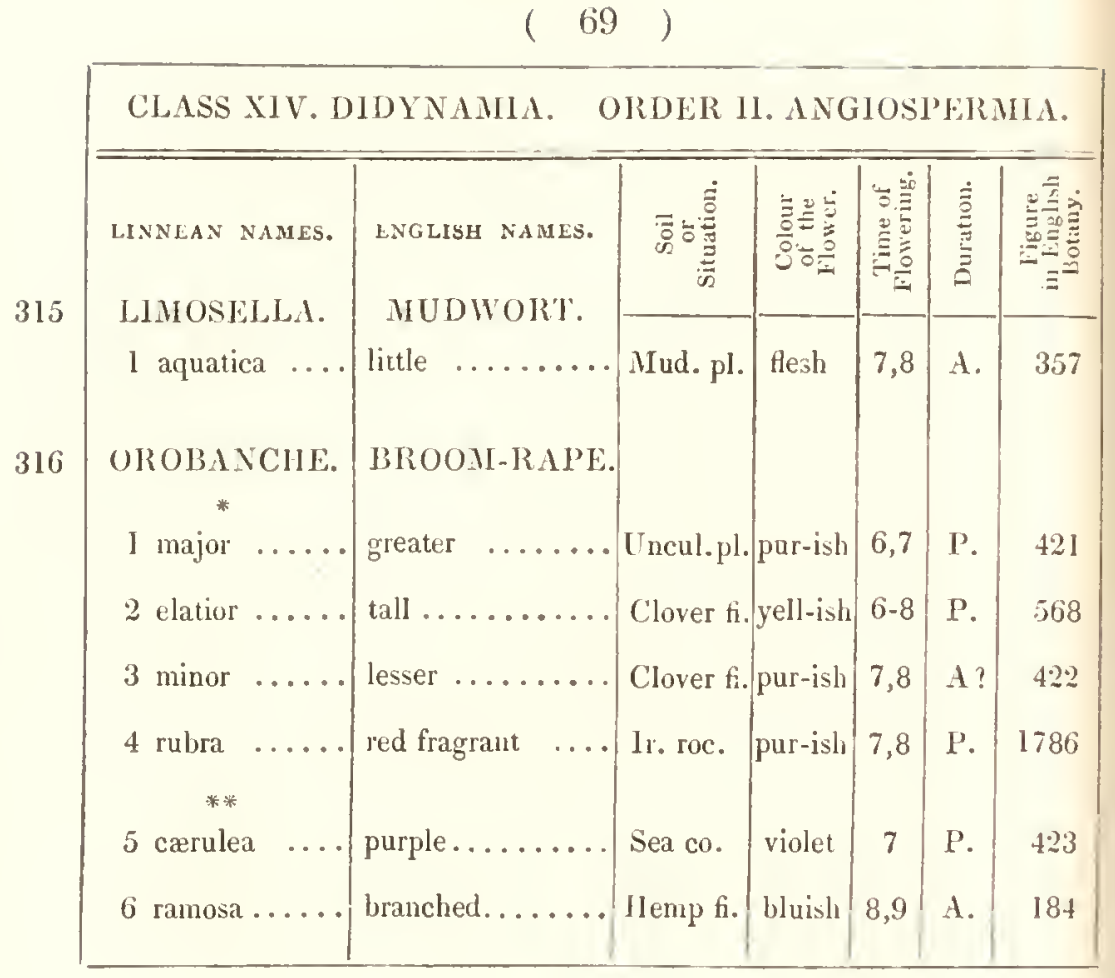


LASS XIV. DHUINAMIA. ORDER II. ANGIOSPERMIA.

SPECIFIC CIIARAETER.

315. LIMOSELLA. Scrophularinex. Juss.

$1 \mathrm{~L}$. lanceolate, somewhat spatulate: petioles twiee as long as the peduncles.

316. OliOBANCIlE. Orobanchere. Vent.

* Bracter solitury.

1 Stem simple: cor, inflated; segments of the lower lip acute, equal: stam. smooth: style pubescent.

2 Stem simple: eor. tubular; segments of the lower lip acute, equal: stam. pubescent: style smooth.

3 Stem simple: cor. tubular; middle segment of the lower lip lobed: stam. fringed: style smooth.

4 Stem simple: cor. tubular; segments of the lower lip blunt, equal: stam. fringed at the base: style partially liairy.

** Bracter by threes.

5 Stem simple: upper lip of the corolla bifid, ineised; lower in three equal segments: style downy.

6 Stem branched: upper lip of the eorolla, bifid; lower equally 3-lobed; segments obtuse, very entire: style smoothish. 


\section{CLASS XV.}

\section{TETRADYNAMIA. Cruciferea. Juss.}

\section{ORDER I. SHLICULOSA.}

\section{* Silicles without valies. .}

330 Cakile. Silicle decidnous, 4-angular, 2-jointerl: each joint l-seeded.

331 Crambe. Silic. dceiduous, 2-jointed; upper joint nearly globular, 1 -seeded; lower joint abortive, like a pedicle.

327 Sfnelzifra. Sicil, nearly entire, compressed, wrinkled, 2-celled.

$$
\text { ** Silicles 2-valied, entire, or nearly entire. }
$$

317 Vella. Silic. tumid, with the leafy ovate style projecting, twiee as long as the valves.

329 Isatis. Silic. deciduous, bordered, 1-celled, 1-seeded. Filum. simple.

318 Subularia. Silic.oral: valves tumid, many-seeded: cotyledons linear.

323 Iutcurssia. Sill. elliptical; valves keeled. Seeds 2 in each cell. Filum. simple.

319 Draba. Silic, oval; valves flattish. Cells many-seeded. Seeds without a margin. Filum. simple.

320 Atrssun. Silic, nearly round; valves flatioh. Cells 1-2-seeded. Filum. often toothed.

321 Camelina. Silic. entire, obovate, smooth; valves tumid, manyseeded. Filum. sinjple.

326 Cocmlearia. Siliv, uearly entire, ovate or roundish, rugose, manyseeded; valives tumid.

****alictes 2-ratred, emarminate or notched.

322 Leproum. Silic. elliptical; valves boat-rhaped. Cells 1-secded, l'et. ectual.

328 InEras. Silic. olrcordate; valves boat-shaped, wingred. Cells l-scriled. Pet. unepual.

325 'Tumasu's. Silic. obcordate, compresed; valves boat-sliaped, often winged. Cells many-seeded. Filum. simple.

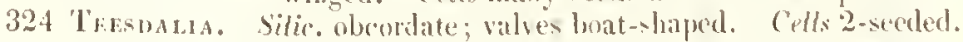
Filum. with a scale at the inside, near the base. 


\section{* Culyr closed.}

5 Raphanus. Silique valveless, jointed, swelling. Seeds globular.

1 Ananis. Silique linear, crowned by the sessile stig; valves roincd or ribbed. Seerls 1-rowed.

2 Turriss. Silique long, 2-cdged; valves ribbed or kceled. Seeds 2-rowed.

6 Barmarea. Silique sipuare-2-edged. Seeds 1-rowed. Cilands within the shorter filam.

37 Ensonval. Silique 4-angular. Seeds without a margin. Stig. capitatc, often 2 -lobed.

8 Cuemanthus. Silique compressed or 2 -edged. Cul. with 2 opposite leafl. ponched at the base. Stig. 2-lobed, lobes sprealing or capitate.

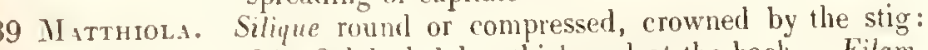
sig. 2-lobed, lobes thickened ai the back. Filam. long, dilated. C'al. with 2 opposite leafl. pouched at the base.

40 Hesperis. Silique angular. Stig. nearly sessile, with converging lobes. Cilumds within the shorter filam. P'et. oblique. Cal. with 2 opposite leaf. pouched at the base. sceds not bordered.

43 Brassica. Silique roundish, with a beak barren or 1-seeded. Seeds globular.

32 Dextaria. Silique linear, bursting elastically from the lase; valves flit, plain, narrower than the partition. Stig. notched. Seeds on flat dilaterl stalks.

\section{* Calyx spreading.}

:33 Cardanise. Silique linear, bursting elastically from the base; valves flat, plain, narrowcr than the partition. Stig. entire. Seeds on capillary stalks.

335 Sisymurus. Silique roundish or angular. Cal. open or somewhat erect. Stig. capitate, notched.

334 Nastuntiun. Silique roundish, oblique; valves concave, ncither ribbed nor keeled. Cul. cqual at the basc. Stig. olotuse, notched.

344 Sinapis. Silique roundish, with a prominent partition. Cal. sprcading horizontally. seeds nearly globular. 
CLASS XV. TETRADYNAMIA. ORDER I. SILICULOSA.

1 aquatica ....

D) RABA.

1 aizoides WHITLOW-GRASS.

2 verna $\ldots . . .$. yellow alpine ... Rocks. $_{\text {yellow }}$

3,4 P.

1271

3 hirta....... common....... Walls .. white simple-haired .... Alp. roc. white

4 incana ...... twisted podded .. Alp. roc. white

5 muralis s spentan

\section{ALYSSUM.}

1 maritimu

ALYSSUM.

CAMELINA. GOLD OF PLEASURE. l sativa $\$ . . .$.

\section{LEPIDIUM.}

I'EPPER-WOR'T)

1 latifolium....

2 ruderale .... broad leaved .... Salt mar. white

3,4

A.

586 $5,6 \quad$ P. 1338

\begin{tabular}{l|l|l}
5,6 & B. & 388
\end{tabular}

3 campestre...

narrow leaved....

4 hirtum ...... hoary field ... Sea co. .....

4 hirtum ......
hairy $\ldots . . .$. lields! IIUTCHINSLA.

IIUTCHINSIA. 
317. VELLA.

L. doubly punatitid: silicles pendulous.

$$
\text { 318. SU13LARIA. }
$$

L. awl-shaped.

\section{J) IBA.}

Scape naked: pet. notched, twice the length of the cal: L. lanceolate, rigid, keeled, fringed.

Scupes miked: pet. bipartite: L. lanceolate, slightly cut, hairy.

Scape 1-leaved: pet. entire: silic. elliptic-oblong: L. lanceolate, somewhat toothed, ciliate; hairs simple.

Stem-leaves numerous, hoary, with interwoven starry hairs: silic. oblong, oblique, longer than the hairy partial stalks.

Stem branched : L. cordate, toothed, hairy: silic. elliptical, obtuse, flat, shorter than the partial stalks.

\section{ALYSSUM.}

Stem somewhat slirubby, much branched, sprearling: I.. lanceolate, acute, entire, loary, with close-pressed liairs: stam. simple: seeds solitary.

\section{CAMLLINA. Ilort. Kew.}

Alrssur, Eng. Bot.

Silic. obovate, bordered, twice as long as the style: L. lanceolate, arrow-shaped: stig. simple.

\section{LEPIDIUM.}

L. ovate, lanceolate, undivided, serrated.

Fl. disudrous, apetalous: 1. lower pinnatifid, toothed; upper linear, very entire.

Silic. scaly, bordered at the top: style very short: stem-leaves arrow-shaped, toothed. Thlaspi, Eng. Brot.

Silic. bairy, bordered at the topl: style long: stem leaves arrowsliaped, slightly tootherl. Thlaspi, Eng. Bot.

\section{:323. HUTCHINSIA. Hort. Keut.}

\section{LeprouUa, Lng. Bot.}

L. pinnate, entire: pet. shorter than the cal : stig. sessile. 
CLASS XV. TEVRAJYNAMIA.

ORISER I. SILICULOSA.

RNGISH NAMLS.

TEESD $\Lambda \mathrm{LI} A$.

THESDALIA.

1 nudicuulis... naked stalked .. Grav. pr. white

$\Lambda$.

327

T'ILASLI.

MTIHIDATE MUSTARD.

1 arvense .... penmy cress

.... Com fi.! white

$6,7 \quad A$.

16.59

2 perfoliatum .. perfoliate ...... Ston.piss! white

4,5

A.

2354

3 alpestre..... alpine

M. past. white

$6,7 \quad P$.

81

+ Bursa-pastoris

shepherd's purse.

Roill si. white :i-11

A.

1485

COCHLEARIA. SCURVYGRASS

1 officinalis....

common

Seil shore white

5

A.

5.51

2 groenlandica .

greenlin

Sce alps

flesh.

A. 12403

3 anglica......

"moglish

........ San shore white

8

A. 5.52

4 danica ......

danish

Sea sho.! white

$b, c$

A.

696

5 Armoracia...

WIat. p!. white

I'.

노년

SFNEBIERA.

IVAR'T'C'RESS.

1 Coronopus...

comining

Romel si. white

$6-8$

d.

1660

\& dirlymil....

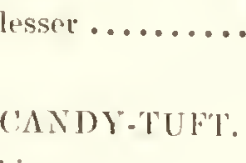

1 :llmiral ..... bitte

Rinbble

white 7

A. $* 2.18$

ISA'T'IS.

IVOA1).

1 tinctorit .... dyers'.

Chal, ti.! white

7

A.

52

C, $\Lambda \mathrm{K} \| \mathrm{l}$.

SLA-ROCKET.

1 mamitima .... common ....... Sean sliore purple $6-9$ 
LASS XV. TETRADYNAMIA. ORDER 1. SILICULOSA.

SPECIHIC CHARACTEH.

324. TEESDALIA. Hort. Kew.

Iberis, Eng. Bot.

Petals unequal.

\section{THLASPI.}

Silic. orbicular, compressed, smooth: L. oblong, toothed, smooth : stem erect.

Silic. obcordate: stem leaves sagittate-cordate, embracing the stem: stem branched: style very short.

Silic. obovate, retuse: stem leaves arrow-shaped: stems simple: style extended.

Hairy : silic. triangular-obcordate: radical leaves pinnatifid.

\section{COCHLEARIA.}

L. radical roundish; on the stems oblong, somewhat sinuated: silic. globosc.

L. kidney-shaped, fleshy, entire; uppermost oblong: silic. globular.

L. radical ovate, entire; on the stems lanceolate, toothed, sessile: silic. elliptical, reticulated with veins.

L. all deltoid, petioled : silicles elliptical, reticulated with reins.

L. radical, oblong, creuate; on the stems lanceolate, cut or entire.

\section{SENEBIERA.}

Silic. entire, crested with little sharp points: style extended : corymb. few-flow: L. pinnatifid, subdivided.

Silic. notched, dirlymous, with reticulated wrinkles: style obsolete: corymb. many-flow. Lepidium, Eng. Rot.

\section{IBERIS.}

Herbaceous: L. lanceolate, acute, slightly toothed: El. in oblong clusters.

\section{ISATIS.}

L. radical, crenate; on the stem sagittate: silic. bluntish, smooth.

330. CAKILE. Hort, kiew.

Bunias, Eng. Bot.

Silic. with 2-edged joints; the upper ones sagittate: L. Aleshy, pinnatifid, obtuse. 
CLASS XV. TLTRADYNAMIA. ORDER I. SILICULOSA.

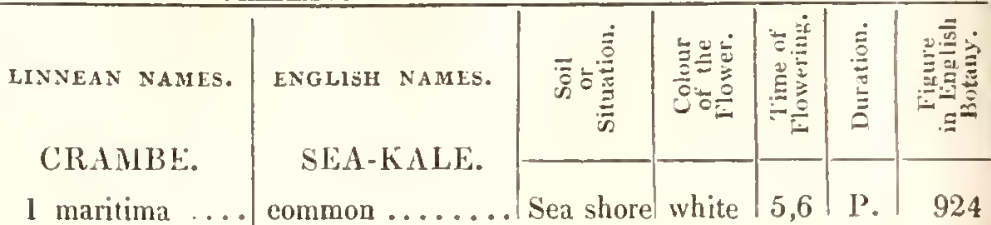

ORDER II. SILIQUOSA.

\section{DENTARIA. CORAL-WORT.}

I bulbifera .... bulbiferous...... Mo.sh.pl! purple $4,5 \mid$ P'. 309

1 bellidifolia .. daisy-leaved .... Sc.alps! white

2 impatiens.... in

impatient........

Alp. Ioc. white

3 hirsuta ..... hairy ......... Moi.sh.p. white

4 pratensis .... nieadow ........ Mea. pas. purple

5,6

5 amara bitter Wat.pl.! white

4,5

A.

80

A. 492

I'. 776

NASTURTIUA. WATER-CRESS. (1)

1 officinale .... eommon .......

2 sylvestre .... ereeping rocket ..

3 terrestre .... annual rocket ....

Rivulets white

Wat.pl. yellow

6,7

6-9

1. ${ }^{*} 855$

Wat.pl. yellow

$6-9$

P. 2324

4 amphibium .. amphibious......

Riv. ban. yellow

A. $* 1747$

6-8 P. *1840

\section{SISYMIBRIUA. ROCKET.}

1 officinale .... hedge-mustard .. W aste gr. yellow 6,7

A. $* 735$

2 Sophia ..... fix-weed.......Wiste gr. yellow

7,8

A. 963

3 Irio ....... London ....... 
ASS XV. TFTRADYNAMIA. ORDER I. SILICULOSA.

\author{
SPECIFIC CHARACTER.
}

331. CRAMIBE.

L. sinuated, undulated, glaucous, smooth as well as the stem : longer filaments toothed.

\title{
ORDER II. SILIQUOSA.
}

332. DENTARIA.

Lower leaves pinnate; upper simple, with axillary bulbs.

\section{CARDAMINE. \\ * I.eaves simple.}

L. simple, ovate, entire; radical ones about one-third the length of the petioles: style short, conical.

$$
\text { ** Leaves pinnute. }
$$

Leaf. lanceolate, cut or entire: stipulæ ciliated.

L. without stipulæ; leaf. roundish-oblong, cut, petioled.

L. without stipulæ: radical leaf. roundish, toothed; stem ones lanceolate: pet. with a tooth upon the claw.

L. without stipula; ; radical leafl. roundish ; stem ones toothed-angular: stem taking root at the base: style obliquely elongated.

\section{NASTURTIUH. \\ Sisyabrum. Fing. Bot.}

L. pinnate: leafl. roundish, lieart-shaped, wavy.

L. pinnatc: leaf. lanceolate, deeply serrated : root crceping.

L. pinnatifid, unequally toothed: root simple: pet. storter than the cal : silique curred.

L. oblong, pinnatifid or serrated: pet. longer than the cal: root fibrous: silique clliptical.

\section{SISYMIBRIUMI.}

Silique close-pressed to the stalk, awl-shaped, downy: L. runcinatc, hairy; stem rough with deflexcd bristles. Erysimum. Fing. Bot. L. doubly pinuatifid, slightly hairy: pet. less than the calyx.

L. runcinate, toothed, nakcd: stcm smooth : siliques crect. 
CLASS XV. TETRADYNAMIA. ORDER II. SILIQUOSA.

336

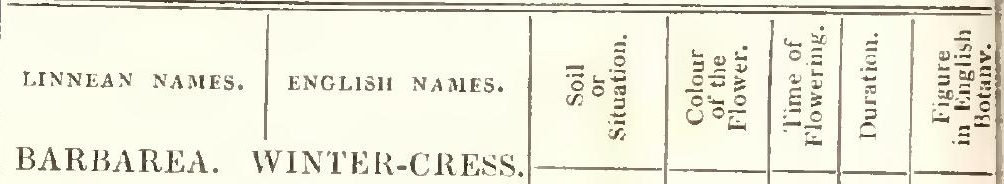

CHEIRANTHUS WALL FLOWER

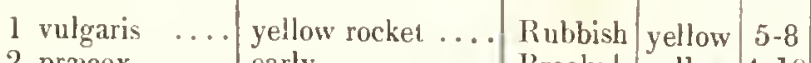

2 pracox..... early ......... Brooks! yellow 4-10

\begin{tabular}{l|l|c|c|c}
\hline & & & & \\
Rubbish & yellow & $5-8$ & P. & $* 443$ \\
Brooks! & yellow & $4-10$ & B. & $* 1129$
\end{tabular}

ER YSLIIUA. HEDGE-MUSTARD.

1 Alliaria.. .9 garlick......... Hedges white 5-7

2 cheiranthoides

\begin{tabular}{ll|l|l} 
White & $5-7$ & A. & 796 \\
\hline yellow & 7 & A. & 942
\end{tabular}

3 orientale ....

treacle.......... Fields.. yellow 7

A. 942

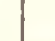

(3)

1 fruticulosus ...

wild

MATTHIOLA.

340

HESPERIS.

DAME'S VIOLET.

1 Matronalis .. common .......P

Old walls yellow 4-6

S.

A. 1804

1 incana ...... ho

2

sinuata......

hoary shrubby....

Cliffs!. purple 5,6

S. $* 1935$

Sea shore violet

8

B. *462

1934

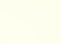

ARABıs.
1 thaliana $\ldots .$.
2 stricta $\ldots . .$.
3 hispicta......

WALL-CRESS.

4 'Turrita common .......W Walls.

white 3,4

A. 901

Bristol rock

Rocks.

cream

l'.

614

alpine tock......

Alp.roc.

purple 6,7

I. 469$$
\text { tower }
$$

Walls! 


\section{SPECIFIC CHARACTER.}

336. BARBAREA.

Errsimum. Eng. Bot.

Lower leaves lyrate; terminal lobe roundish; upper obovate, toothed. Lower leaves lyrate; upper pinnatifid; segm. linear-oblong, entire.

\section{ERYSIMUM.}

L. heart-shaped, broadly toothed, stalked.

L. lanceolate, slightly toothed, roughish with close forked bristles: siliques erect: pcdicles when in fruit spreading.

L. elliptic-heart-shapcd, obtuse, clasping the stem, smooth, entire; radical ones obovate. Brussica. Eng. Bot.

\section{CHEIRANTHUS.}

L. lanceolatc, acute, hoary beneath, with very simple appressed pubescence: stem shrubby, branches angular: style prominent.

\section{MATTHIOLA. \\ Cheiranthus. Eng. Bot.}

L. lanceolate, obtuse, entire, hoary: siliques without glands: stem shrubby, upright, branched.

L. downy, glandular, obtuse, sinuated : those on the branches entire: siliques rough with prominent glands: stem herbaceous, spreading.

\section{HESPERIS.}

Stem erect: L. ovate-lanceolate, toothed: petals obtuse: partial flower-stalks the length of the calyx.

\section{ARABIS.}

I L. hairy, more or less toothed; radical ones petioled: stem branched: siliques ascending: stam. not much shorter than the petals.

2 L. toothed, obtuse, rough; radical ones somewhat lyrate: stems hispid: petals erect: cal. smooth.

B Radical leaves runcinate-lyrate, rough; stem ones lanceolate: pet. spreading: root branched at the crown. Cardamine hastulata. Eng. Bot.

4 L. embracing the stem: siliques bent backward, flat, linear, with an incrassated margin: flower-stalks the length of the calyx; each with a leafy bractca. 
CLASS XV. TETRADYNAIIA.

ORDER II. SILIQUOSA.

| tol to

LINNEAN NAMFS.

ARABIS.

5 ciliata EYGLISH NAMES.

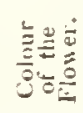

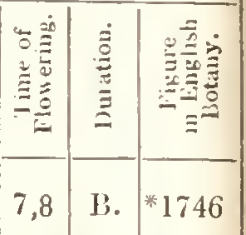

TLRRITIS.

BRASSICA.

I campestris ... fiel

CABBAGE.

2 Napus

WALL-CRESS.

fringed ....... Ir.shores! white

hairy

Rocks.. white

P.

*587

\section{IS. TOWER-MUSTARD.}

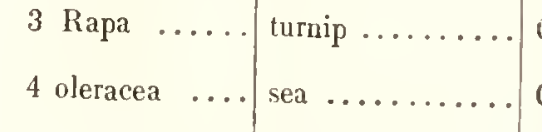
- smooth ...... Grav. pa. white

5,6

A.

777

Corn fi. yellow

Fields.. yellow

6,7

A.

2234

Dit. ban. yellow

B.

2146

5

monensis .... dwarf sea ..... sea shore yellow

Cliffs .. yellow

B.

2176

$5,6) B$.

637

$6,7 \quad 1$

*962

\section{SINAPIS.}

I arvensis ....

NUSTARI).

2 alba

white

ock .

Corn fi. yellow

5

A. 1748 Corn A. yellow 6 A.

1677

3 nigra....... common ......... Bor. of ti. yellow 6,7

$\Lambda$.

969

4 tenuifolia .... fine-leaved.

.

Walls .. yellow $6-10$

P.

$* 225$

RAPHANUS.

1 Rapharistiun sand San. pl. yellow 8 - 10

A. $* 1090$

2 maritimus. 
SPECIFIC CHARACTER.

\section{ARABIS.}

L. somcwhat toothed, smooth, fringed, bearded; radical ones obovate; the rest elliptical, half clasping the stem: stem simplc. Turritis alpina. Eng. Bot.

L. rough: stem hairy, hairs sinple and spreading: siliques erect with slightly kceled valves. Turritis. Eng. Bot.

\section{TURRITIS.}

I. radical, toothed, rough ; on the stem entire, smooth and embracing.

\section{BRASSICA.}

Root and stem slender: L. cordate, acuminate, glaucous, embracing the stem; lowcr ones lyrate, toothed, somcivhat rough.

Root caulescent, fusiform: L. smooth; upper cordate-lanceolate, embracing the stem; lower ones lyrate, toothed.

Root caulescent, orbicular, depressed, fleshy: radical leaves lyrate, rough; those on the stem very entire, smooth.

Root caulescent, cylind. fleny: L. all smooth, glaucous, waved or lobed: silique without a beak.

L. pinnatifid: lobes oblong, unequally toothed: stem smooth: siliques 4-angular; beak simple, 2-3-seeded. Sisymbrium. Ling. Bot.

\section{SINAPIS.}

1. Siliques many-angled, rugged, longer than the 2-edged beak: L. ovate, somewhat lyrate.

2 Siliques hispid, rugged, spreading, shorter than the 2-edged beak: L. lyrate.

3 Siliques smooth, square, appressed to the stem: upper lcaves linearlanceolate, cntire, smooth; lower, lyrate.

4 Siliques linear, erect, on spreading stalks, slightly beakcd: seeds 2-ranked: L. lanceolate, acute, pinnatifid or bipinnatifid: stem smooth. Sisymbrium. Eng. Bot.

5 Siliques linear, ascending, on spreading stalks, slightly beakcd : seeds 2-ranked: L. sinuated, smooth: stem rough with reflexed bristles. Sisymlrium. Eng. Bot.

\section{RAPHANUS.}

1 Siliques jointed, striated, 1-celled: L. simply lyrate.

2 Siliques jointed, deeply furrowcd, 1-ccllcd: L. radical, interruptedly lyrate, serrated. 



\title{
CLASS XVI.
}

\author{
M O N A DELPHIA.
}

\section{ORDER I. PENTANDRIA.}

46 Erodius. Pistil 1. Cal.5-leaved. Cor.5-pet. Fruit5-grained, beaked; beak spirally twisted, bearded on the inside. Lysimachia 1. Limum, Geranium 8.

\section{ORDER II. DECANDRIA.}

-47 Geranium. Pistil 1. Cal.5-leaved. Cor.5-pet. regular. Fruit 5 -grained, beaked; beak recurved, naked, simple. Oxalis. Spartium. Genista. Anthyllis. Ulex. Ononis.

\section{ORDER III. POLYANDRIA.}

350 Lavatera. Pistils many. Cal. double, outer 3-cleft. Caps. whorled, 1-seeded.

349 Niava. Pistils many. Cal. double, outer 3-leaved. Caps. whorled, 1-seeded.

348 Azthea. Pistils many. Cal. double, outer 9-cleft. Caps. whorled, 1-seeded. 


\section{CLASS XVI. MONADELPIIA. ORDER I. PENTANDRIA.}

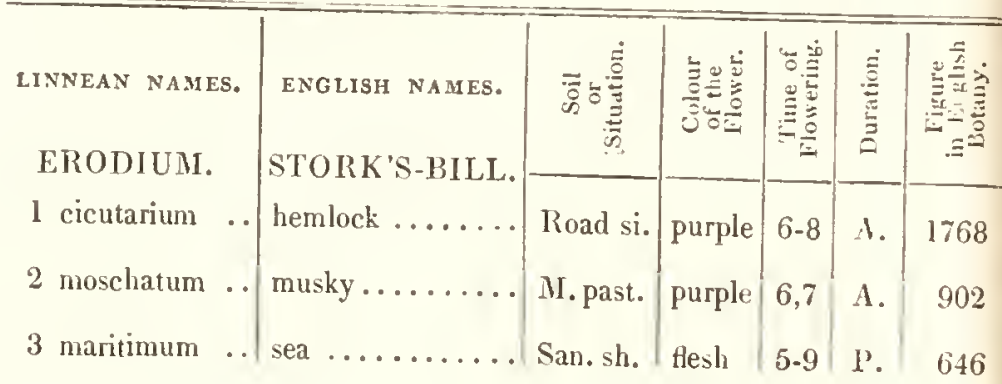

ORDER II. DECANDRIA.

\begin{tabular}{|c|c|c|c|c|c|}
\hline 1 phæum....... & dusky .......... & M. thick. & blood & 5,6 & P. \\
\hline 2 nodosum ... & knotty......... & Mount.! & purple & 5,8 & P. \\
\hline 3 sylvaticum & wood .. & MI. thick. & purple & 6,7 & P. \\
\hline 4 pratense .... & crowfoot-leaved & Mea. pas. & blue & 6,7 & r. \\
\hline 5 robertianum .. & stinking .. & Ban. Wal. & purple & $5-10$ & A. \\
\hline 6 lucidum $\ldots$ & shining $\ldots$ & Stony pl. & rose.. & $5-8$ & A. \\
\hline 7 molle ... & dove's foot. & Waste gr. & purple & $4-8$ & A. \\
\hline 8 pusilluin $\ldots$ & small-flowered & Waste gr. & purple & $6-9$ & A. \\
\hline 9 pyrenaicum .. & mountain . & Mea. pas. & purple & 6,7 & P. \\
\hline 10 rotundifolium & round-leaved & Waste gr. & rose.. & 6,7 & A. \\
\hline Il dissectum.... & jagged-leaved . & Waste gr. & purple & 5,6 & $\Lambda$ \\
\hline 12 columbinum.. & long-staiked & Chal.ban. & rose.. & 6,7 & $A$ \\
\hline 3 sanguineum. & bloody.......... & Rocks.. & blood & $7-9$ & P. \\
\hline
\end{tabular}


ILASS IVI. MUNADELPHIA. ORDER I. PLNTANDRIA. SWECIFIC CHARACTER.

346. ERODIUM. Geraniacea. Juss.

Stems procumbent, hairy: pcdunc. many-flow: L. pinnate; leafl. sessile, pinnatifid, cut: stam. simple.

Stens depressed, hairy : pedunc. many-flow : L. pinnate; lcafl. almost sessile, elliptical, unequally cut: perfect stam. toothed at the base.

Pedunc, about 3-flow: L. simple, cordate, cut, crenate, rough : stems depressed, hairy.

\section{ORDER II. DECANDRIA.}

\section{GERANIUM. Geraniacer. Juss.}

Pedunc. 2-flow. panicled, erect: cal. somewhat awned: caps, keeled, lispid at the base, wrinkled at the summit: stam. liairy.

2 Pedunc. 2-flow: L. opposite, 5 or 3-lobed, serrated: caps. even, downy all over.

3 Pedunc. 2-flow. somewhat corymbose: L. 5-7 lobes, deeply notched, and serrated: caps. all hairy : stam. subulate, fringed.

11 Pedunc. 2-flow: 1. 5-7 parted, notched and serrated: caps. all hairy: stam. deltoid at the base.

5 Pedunc. 2-flow: L. somewliat pedate, pinnatifid, 5-angled: cal. 10angled: caps. rugose.

6 Pedunc. 2-flow: L. 5-lobed, roundish: cal. pyramidal, transversely wrinkled: caps. wrinkled, 3-keeled.

7 Pedunc. 2-flow. alternate, opposite the leaves: L. roundish, lobed, downy: caps. wrinkled, smooth: seeds without dots.

8 Pedunc. 2-flow: L. reniform, palmate, cut, downy: caps. even, hairy: seeds smooth: anthers only 5 .

9 Pedunc. 2-flow: pet. twice as long as the cal: L. reniform, lobed: caps. even, slightly pubescent: seeds smooth.

0 Pedunc. 2-flow: petals entire: L. reniform, cut, downy: caps. hairy: seeds reticulated.

1 Pedunc. 2-flow: petals notched : L.5-parted, laciniated: caps. hairy: seeds reticulated.

2 Pedunc. 2-flow. thrice as long as the leaves: L. 5-parted, many-cleft: caps. smooth: seeds reticulated.

13 Pedunc. 1-How: L. 5-7-parted: lobes 3-cleft, roundish: caps.bristly at the apcx: seed minutely wrinkled. 


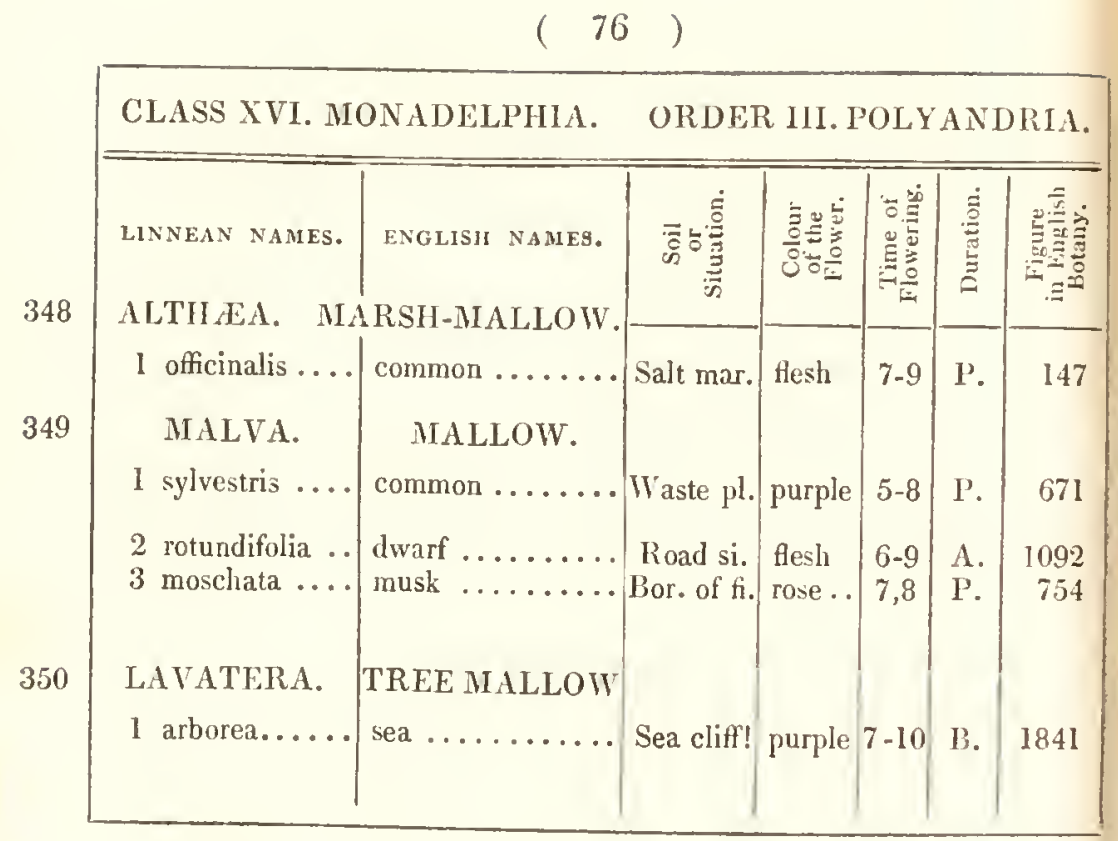




\section{MSE XII.MONADELPHIA ORDFR 111. POIAANDHA.}

SPIC"IFIC CHARACIFR.

348. ALTHEA. Malvarea. Juss.

IL. simple, downy, stightly 5-lobed.

3.19. MAL NA. Malvacea. Jess.

:Stem erect, herbaceous: L. 7 -lobed, acute: perlunc. and petioles hiiirs.

Stems prostrate : L. cordate-orbicular, 5-lobed : fruit stalks sleclining. L. radical, reniform, cut; on the stem 5-parted, pinnate-multifid: cal. hairy, its outer leares linear-lanceolate.

\section{LATATERA. Malvacer. Juss.}

Stem arborescent: 1. 7 -angled, downy, plaited: pedunc. axillary, crowded, 1 -flow. 


\title{
CLASS XVII.
}

\author{
DIADELPHIA.
}

ORDER I. HEXANDRHA.

3,1 Fumana. Cal. z-leaved. Cor. ringent, gribbous at the base, nectiriferous. Filam. with 3 anthers.

\section{ORDER II. OCTANDRIA.}

35 Polygala. Cal. 5-leaved; " leafl. wing-like, coloured. (or. standird cylindrical. Pod obcordate, 2, -celled. Seeds solitiry, crested.

\section{ORDER III. DECANDRIA.}

* Stam. all comected, or monadelphons; tube frequently clefi ubove. 353 Spartiua. Filam. united in cue undivided tube: Stig. lateral linear, hairy. Legume flat.

354 Gexista. Pistil depressing the keel. Standard reflexed. Stig. terminal.

355 Ulex. Cal. 2-leaved. Pods scarcely longer than the callyx.

357 Axmylus. Cal. turgid, inclosing the pod.

356 Oxonss. Cal.5-plarted. Pods rhomboid, sessile. Stundard striated.

** Stigma or style, pubescent, (without the marlis of the first division.)

359 Oronus. Style linear, almost cylindrical. Stig. downy above.

358 Prsum. Style triangulin, ind downy above.

:360 Latnyous. Style flit and lowny ibove.

361 Vicia. Style bearded under the stigmi.

362 Envon. Stigma capitite, pubescent on every part.

**** Pod "L-celled, (uithont the marls of the first und second divisions.) $365^{\circ}$ Astragalus. l'od 2-celled, gibbous.

***** Por generally 1-seeded, (withont the marks of the former dixisions.)

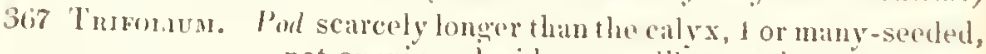
not openinger, deciluous. litom. capitate.

367 Mtincors. Legune 1 or fow-seeded, indehiscent, lonver thin the calyx. l'etuls distiuct, deciduous. 
ASS XVII, DHDELIJIA. ORDER III, DECANDRIA.

**: Pod either jointed, or spiral, (without the marks of the former dirisions.)

Ineysaruir. Port joints compressed, 1-seeded. heel very obtuse.

Onsmupes. Pod jounted, bowed, cylindrical; joints 1 -seeded. heel roumled.

Hiprochirts. P'od compressed, membranous incurved; one edge with many curver notches.

Nledicaco. Pod spiral, compressed, somewhat membranous. Pistil pressing down the keel.

****** Prol l-celled, many-seeded, (without the murks of the former dirisions.)

Lotus. Puct cylindrical, spongy within. Wings converging longitudinally upwards. Filtom. partly dilated. 
CLASS XVII. DIADELIIIIA.

ORDER I. IIEXANDRIA.

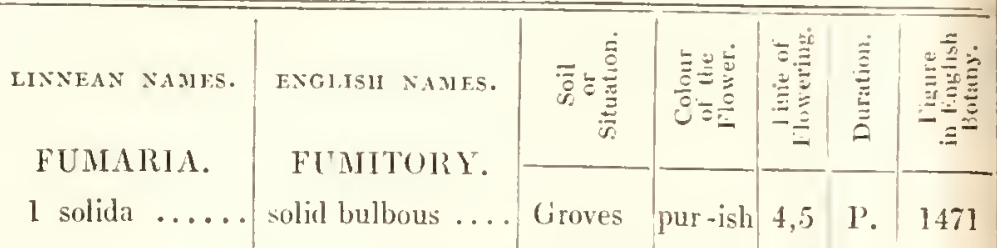

2 Iutea....... yellow......... Old walls! yellow 5 I'. 588

\begin{tabular}{ll|l|l|l|l|l}
3 officinalis ... common ........ & Cult.gr. & rose .. & $5-8$ & 1. & 589
\end{tabular}

\begin{tabular}{ll|l|l|l|l|l|l|}
4 parviflora .... & small flowered .. & Corn fi. & rose . & 8,9 & А. & 590
\end{tabular}

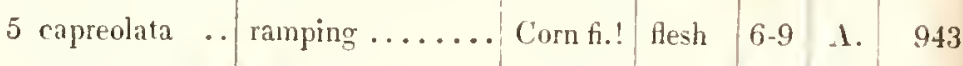

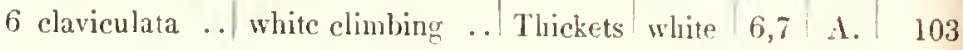

\section{ORDER II. OCTANDRIA.}

POLYGALA. MILK-WORT.

1 vulgaris

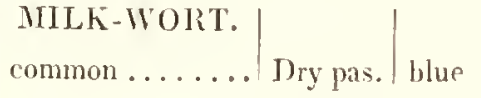

$|6,7| \mathrm{P}$.

76

ORDER III. DECANITRIA.

\section{SPARTIUN. | BROOM.}

1 scoparium .. GENISTA. GRELN-WELD.

1 tinctoria .... dyer's ........ Dry pas. yellow 7,8 s.

2 pilosa ..... hairy ........ San. hea.! yellow $5 ₫ 9$

3 anglica..... ncedle......... Moi. hea. yellow 5,6

S. $\quad 132$

ULEX.

I curopirus ....

FU1:\%L. 
SPECIFIC CHARACTER.

\section{3i). FUMARIA. Frmariacer. DE C.}

atem simple, erect: 1. liternate: bract. palmate, longer thai the pedune.

Pods almost cylind. shorter than the pedune: stems angular: braet. minute: spur very short, rounded.

Cluster lax: porls I-seeded, globose, notched: stem diffuse: leath. with dilated segments.

Cluster lax: pods I-seeded, globose, acuminate: stem diffuse: lcafl. with linear ehamelled segments.

Cluster lax: pods 1-seeded, globose: stem elimbing: petioles twisted: leat. wedge-shaped, lobed.

IPods lanceolate, undulated: stem climbing : petioles tendrilled.

\section{OLDER II. OCTANDRIA.}

352. POLYGALA. Polygaler. Juss.

Fl. racemose, erested: bract. 3. at the base of each perlune. deciduous: wings about equal to the corolla: stems herbaceous, simple, partly procumbent: L. linear lanceolate.

ORDER III. DECANDRIA. Ieguminoser, IISS.

\section{SPARTIUNI.}

L. ternate, or' solitary : branches thornless, angular: pod. fringed.

\section{GENISTA.}

L. lanceolate, sinnotlı: branehes eylind. striated, ereet, thornless.

L. obovate-lanceolate, obtuse, hairy beneath : sten tulıercled, decumbent, without thorns: cal. and pedune. silky.

spines simple: fowering luranches without spines: 1. ovate-lanceolate.

$$
\text { 355. ILEX. }
$$

Calyx-tecth indistinet, converging: bract. ovate, loose: branches erect.

Calyx-teeth lanceolate, spreading: brart. minute, closc-pressed: branclies reclining. 


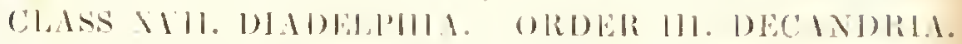

IINEAN NAMES.

\begin{tabular}{|c|c|c|c|c|}
\hline ENGLISU NAMES. & 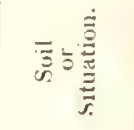 & 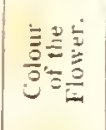 & 标 & 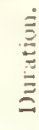 \\
\hline $\begin{array}{l}\text { R Jist l] ARlioll } \\
\text { common ....... }\end{array}$ & Bar pas. & rose .. & $6-8$ & $P^{\prime}$ \\
\hline
\end{tabular}

ANTHYLLIS.

KIDNEY-IFCII.

I vulneraria ...

\section{Kany fis}

\section{PISUM.}

I maritinum

PFA.

\section{Oli()]3U.}

1 tuberosus....

2 niger

3 sylvaticus....

\section{ルTHY}

舟

1 Aphaca ....

2 Nissolia ....

yellow vetchling.

- Sea shore blue

Clial. pas. yellow

$6-8$

$1 '$.

104

sea.......

BITER VECH

heath .

Ileaths

blaek

M. pas.! purple

wood

NI. wo!

\section{LATIIIRIS.}

3 hirsulus crimson grass

$$
\text { *** }
$$

4 pratensis .... ye

5 sylvestris .... in

yellow meadow.. Mea. jas. yellow marrow-leaved.... Mloi. wo. purple

6 latifolius everlanting pea. rough polderl .... Hor.olfi.! purple 7

1.

l. $\quad 1046$

7 palustris 111

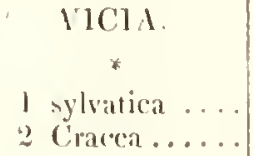

VITUI

wood Ilonds! rove. 
SPECIFIC CHARACTHR.

3.56. UNONIS.

Stem hairy: branclies at length spinous: Fl. solitary, or in pairs: L. generally simple, entire behind.

357. ANTIIYLLIS.

Herbaceous : L. pinnate, unequal: heads of flowers in pairs.

\section{PISUM.}

Petioles thattish uhove: stem angular, stip. sagittate: peduncles many-flow.

\section{OROBUS.}

L. pinnate, elliptic-lanceolate: stip. semi-sagittate, toothed at the base: stem simple, erect.

L. pinnate : leafl.elliptic-lanceolate : stip.linear-awl-sliaped, simple, entire: sten branched, angular, erect.

Stems decumbent, hirsute, branched : leatl. mumerous.

\section{LATHYRUS.}

* Peduncles 1-flowered.

Tendrils leafless: stip. sagittate-cordate.

Pedunc. mostly 1-flow: L. simple, without tendrils: stip. awlslaped.

\section{** Peduncles 2-flowered.}

Tendrils 2-leaved ; leafl.linear-lanceolate : pods luairy: seeds rough.

**** Peduncles many-flow.

4. Tendrils 2-leaved, mostly simple; leatlets lanceolate.

i) Tendrils 2-leaved; leatlets ensiform : stem winged.

Tendrils 2-leaved: leaflets elliptical: stem winged.

Tendrils many-leaved: stip. lanceolate, acuminate.

\section{VICIA.}

* Pedunc. elongated, many-flow.

1 Leafl. elliptical : stip. lunate, toothed.

2 Fl. imbricated: leafl. lanceolate, pubescent : stip. semi-sagittate, mostly entire.

** Flowers axillary, almost sessile.

Pods mostly in pairs, nearly erect: lower leaves blunt: stip. marked: seeds smooth, orbicular. 
CLASS XVII. DIADELPHIA. ORDER III. DECANDRIA.

\begin{tabular}{|c|c|c|c|c|c|c|}
\hline LINNEAN NAMES. & ENGLISII NAMES. & 家慧 & 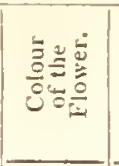 & 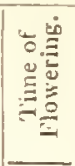 & 递 & 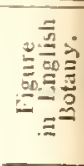 \\
\hline 4 angustifolia.. & narrow-leaved & Grav. pas & crimso. & 6 & A ? & \\
\hline 5 lathyroides.. & spring....... & Fallow $\mathrm{f}$. & blue & 4,5 & A. & \\
\hline 6 lutea $\ldots .$. & rough-podkled & Sea shore & yellow & 8 & P. & \\
\hline 7 lyybrida .... & hairy flowered yel. & T'hickets! & yellow & 6,7 & P. & \\
\hline 8 lrevigata ... & smooth-podded .. & Sea slı.! & purple & 7,8 & P. & \\
\hline 9 sepium $\ldots$ & bush $\ldots . .$. & Hedges & blue & 5,6 & P. & \\
\hline 10 bythynica .. & rough-podcled & Bush.pl.! & purple & 7,8 & P. & \\
\hline ERVUM. & & & & & & \\
\hline $\begin{array}{l}1 \text { tetraspermum } \\
2 \text { hirsutum.... }\end{array}$ & $\begin{array}{l}\text { smooth ........ } \\
\text { hairy .......... }\end{array}$ & $\begin{array}{l}\text { Corn fi. } \\
\text { Corn fi. }\end{array}$ & $\begin{array}{l}\text { purple } \\
\text { blue }\end{array}$ & $\begin{array}{l}6,7 \\
6-8\end{array}$ & A. & $\begin{array}{r}122 \\
97\end{array}$ \\
\hline $\begin{array}{l}\text { ORNITHOPUS. } \\
1 \text { perpusillus.. }\end{array}$ & $\begin{array}{l}\text { BIRD'S-FOOT. } \\
\text { common ....... }\end{array}$ & Dry pas. & redlis. & 5 & A. & 30 \\
\hline HIPPOCREPIS. & HORSE-SHOE-V & ETCH. & & & & \\
\hline 1 comosa..... & tufted ........... & Chal. hil. & yellow & $5-8$ & P. & \\
\hline $\begin{array}{l}\text { HEDYSARUM. } \\
1 \text { Onobrychis.. }\end{array}$ & $\begin{array}{c}\text { SAINTFOIN. } \\
\text { common ........ }\end{array}$ & Chal.pas. & rose.. & 6,7 & P. & \\
\hline $\begin{array}{l}\text { ASTRAGALUS. } \\
1 \text { glycyphyllos. }\end{array}$ & $\begin{array}{l}\text { MILK-VETCH. } \\
\text { sweet .......... }\end{array}$ & Chal. wo. & yellow & 6 & $\mathbf{P}$. & 26 \\
\hline 2 liypoglottis .. & purple mountain. & San. hea. & purple & 6,7 & P. & \\
\hline 3 uralensis & hairy mountain .. & Sc. alps & purple & 7 & P. & 16 \\
\hline 4 campestris & yellowishmountain & IIighlan. & varieg. & 7 & P. & 25 \\
\hline
\end{tabular}

1 perpusillns..

\section{HIPPOCREPIS.}




\section{$79 \quad)$}

ILASS IVII. DIADELPIHA. ORDER III. DECANDRIA.

SPECIIIC CHARACTER.

\section{VICIA.}

Pods solitary : Leafl.linear; lowcr ones obcordate: stip. with a pale depression beneatll: seeds orbicular, smooth.

Pous with a blackish depression beneath, solitary, smooth: L. about 3-pair; lowermost blunt: seeds tubereled: tendrils simple, shorter thin the leaflets.

Pods solitary, hairy, reflexed: stems diffuse : stip. coloured: standard smooth.

Pods solitary, reflexed, hairy : standard rillous : leafl. abrupt: stip. ovate, unstimed.

Pods solitary, reflexed, smooth: stems nearly erect: $L$. very smooth: stip. cloven, unstained.

Pods on stalks, about four together, erect, smooth: leafl. ovate, obtuse: upper ones gradually smaller.

Pods on stillis, solitary, erect, lough : leafl. 2-pair, elliptic-lanceolate : stip. toothed, literally.

\section{ERVUM.}

Pedunc. 2-flow: pods smooth, 4-seeded : leafl. oblong, obtuse. Pedunc. many-flow: pods hairy, 2-seeded : leafl, abrupt.

\section{ORNITHOPUS.}

L. pinnate: Fl. capitate, accompanied by a leaf : pods incurved, beaded.

\section{HIPPOCREPIS.}

Pods umbellate, rough; their joiuts neither dilated, nor bordered.

\section{IIEDYSARUMI.}

I L. pinnate: pods 1-seeded, aculeate: wings of the cor. as long as the calyx: stem elongated.

\section{6. $\Lambda$ STRAGALUS.}

I Caulescent, prostrate : pods almost triangular, bowed : L. longer than the peduncle: leafl. ovate.

Caulescent, prostrate: lil. calpitate: porls ovate, hairy, channelled ou the back, hooked at the ipex: leafl. blunt.

Stemless: stalk erect, longer than the leaves: pods oblong, inflated, villous, orect: leafl. ovite, ncute, silky : cal. silky.

Stemless: stalk iscending: leafl. lanceolate, acute: pods ovate, inflated, villous, rrect. 


\section{CLASS XVII. DIADELPHIA. ORDER III. I)ECANDRIA.}

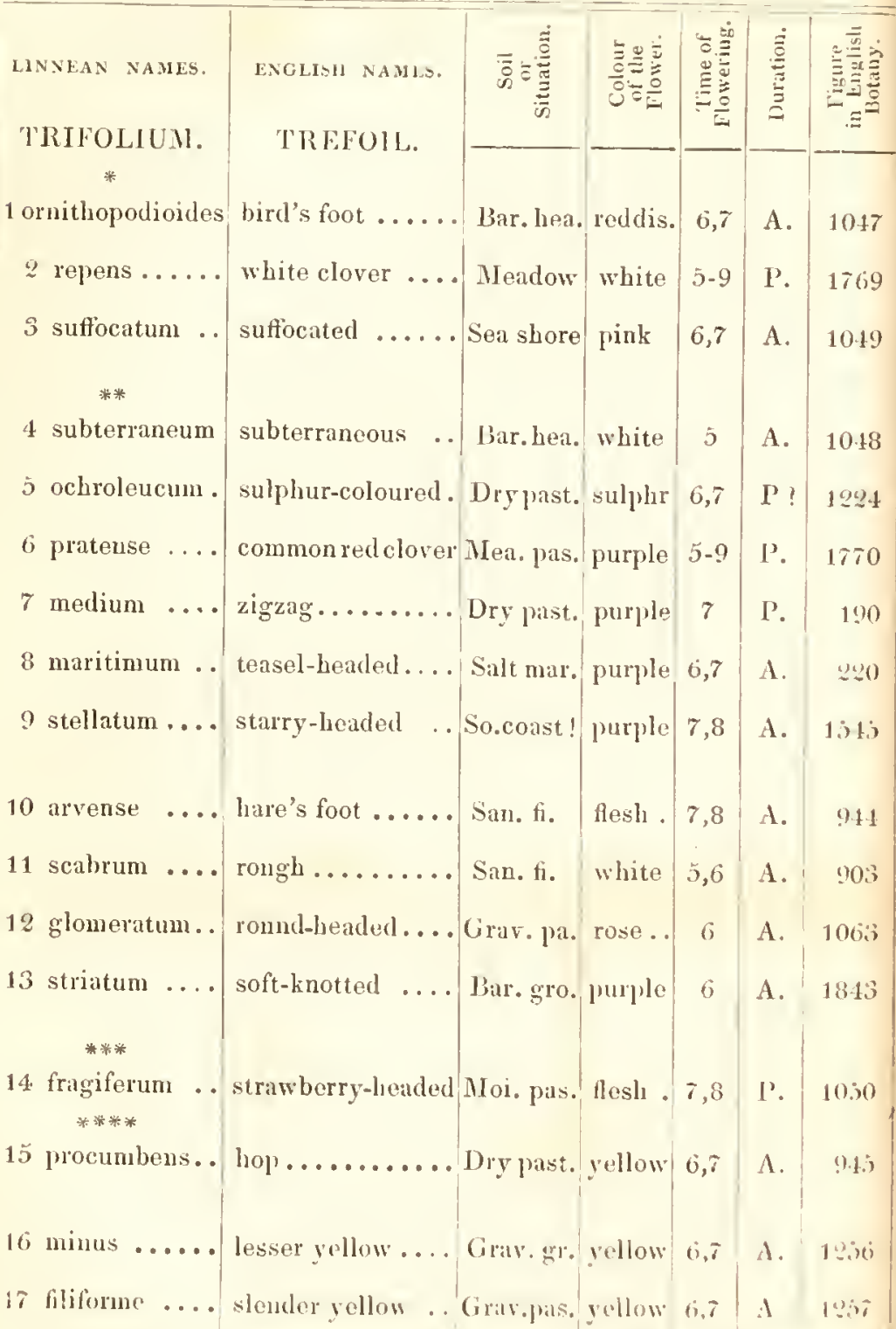


SPLCIFIC CIIARACTEK.

\section{TRIFOLIUM.}

\section{* Flowers capitate. Seeds several.}

Pods naked, 8-sceded, mostly 3-together, twice as long as the calyx: stems decliuing.

Heads globose: Fl. somewhat stalked: pods t-seeded: stems creeping, solid.

Heads sessile, lateral, roundish : pod concealed, 22-seeded : cillyxteeth lanceolate, acute, recurved, longer than the corolla.

\section{** Calyxes grenerally rillous. Seeds single.}

Heads villous, about 4 -flow: involucre central, reflexed, rigid, star-like, embracing the fruit.

Head villous: stem erect, pubescent: lower leatl. obcordate : lower tooth of the calyx very long.

Spikes dense : stems ascending : cor. unequal : cal, with four of the teeth equal : stip. awned.

Spikes loose : stems flexuose, branched : cor. neirly equal : stip. linear, awl-shaped: culyx teeth, 2 upper the shortest.

Spikes hairy, globose: cal. teeth leafy, at length sprending : stip. lanceolate : leafl, obovate.

Spikes hairy: stip. elliptical: cal. teeth longer than the corolla; after flowering, dilated, leafy, reticulated, and spreading : tube closed; stem diffinse : leafl. obcordate.

Spikes very villous, cylind: stip. lanceolate, bristle-pointed : calyxtecth setaceous, longer than the cor: leaf, obovate-linear.

Heads sessile, lateral, ovate : calyx-teeth unequal, lanceolate, rigid, finally recurved: stems procumbent.

Heads hemispherical, sessile, lateral, smooth: calyx-teeth cordate, reflexed, veiny: stcms prostrate.

3 Heads sessile, lateral and terminal, ovate: call elliptical, hairy, furrowed; teeth setaceous: stems procumbcnt.

\section{*** Calyxes inflated, ventricose.}

4 Heads roundish: cal. inflated, 2-toothed, reflexch : stcms creeping.

***** Standards deflexed, al length scuriose.

Spikcs oval, imbricated : standard deflexed, pcrmanent, furrowed: stems procumbent: leifl. obovate: common petiole longest at the basc.

16 Spikes licmispherical: perhunc, straight: standard nearly smooth: stcms prostrite: rommon petiole shortest at the bilse.

Spibes of fow-flow. lax : pedunc. waved: standard smooth. stems prostrate. frafl. all nearly sessile. 


\section{CLASS XVII. DIADEIPHIA.}

OLDER III. DFCANDRIA.

LINNEAN NAMES.

18 resupinatum .

'TREFOIL. reversed...

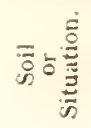

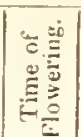

咅

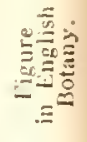

\section{MELILO'TUS.}

1 officinale ....

MELILO'T.

2 leucantha....

..Meadows

LO'TUS.

1 corniculatus.

2

inajor... .

3 decumbens...

4 angustissimus

BIRD'S-FOO'T' 'TREFOIL. con

common

white-flowered ..

Corn fi.

white

7,8

A ?

A.

1340

?

1. .

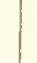

greater ........ W

Pasture. yellow

$6-8$

P.

2090

P.

2091

P.

spreading ...... Meadows yellow

$$
\text { sl }
$$

Sea co. yellow

5,6

A.

925

\section{MEDICAGO.}

1 sativa $\$ \ldots . .$.

\& fitcata......

3 lupulina ....

lucerne $\ldots \ldots \ldots$ Mlea pas. violet
yellow $\ldots \ldots$
.... Bor. of fi. yellow

MEDICK.

nonesuch ..... Pasture yellow

4 inaculata ....

spotted

Grav.pas. yellow

5 muricata ... flat-toothed ...

Sen co

yellow

6,7

P.

1749

P. 1016

A.

971

A. $* 1610$

6 minim:

\section{Sin. fi.! yellow 5,6}

A. 


\section{TRIFOLIUM.}

Heads hemispherical, at length globose : cor. resupinate : cal. after flowering membranaceous, reticulated, inflated, hairy, acute : 8 of the teeth longer, patent: leaflets ovate: stem prostrate.

\section{7*. MELILOTUS.}

Clusters unilateral: pods 2-seeded, rugose, ncute, hairy: stem erect : stip. awl-shaped.

Pods 2-seeded, ovate wrinkled: rncemes lax : cor.twice as long as the calyx: keel and wings shorter than standard: stem erect.

\section{LOTUS.}

Heads depressed, few-flow : stems decumbent, pithy: pods cylind. spreading: claw of the standard obovate: filam. all dilated. lleads depressed, many-flow: stems erect, hollow : pods cylind. drooping : claw of the standard linear: shorter filam. not dilated. lleads few-flow: stems recumbent, nearly solid: pods cylind. 2edged: cal. laiiry; its teeth shorter than the tube.

4 Fl. solitary or in pairs: stems much branched, prostrate, hollow : pods 2-edged, slender: cal. loosely hairy; teetl fringed, twice the length of the tube.

\section{MEDICAGO.}

11 Clusters upright: pods twisted: stem erect, smooth.

2 Clusters upright; pods lunate : stem procumbent.

3 Spikes ovate: pods reniform, veiny, wrinkled, 1 -seeded : stem procumbent.

4 Pedunc. 2 or 3-flow: leafl. obcordate, spotted: stip. dilated : pods spiral, depressed, fringed with long bristles. M. polymorpha, Eng. Bot.

5 Pedunc. 2 or 3 -flow: stip. deeply toothed, hairy: leafl, obovate, hairy : pods depressed, with short radiating teeth. Phk. Phyt. t. 113. f. 6 .

6 Pedunc. many-flow: stip. half-ovate, undivided: leaff. obovate, hairy: pods orbicular, hairy, with divaricated hooked spines. Flor. Dan. 1. 211. 



\section{CLASS XVIII.}

\section{POLY A DELPHIA.}

\section{ORIER I. POLIANDRIA.}

370 Hrpraicus. Cal.5-parted, inferior. Pet. 5 . Filam. in 3 or 5 sets, united at the base. C'ups. many-seeded. 
CLASS XVIII. POLYADELPIHA. ORDER I. POLYANBRIA.

\begin{tabular}{|c|c|c|c|c|c|c|}
\hline LINNLAN NANES. & EXGLISI XАM & 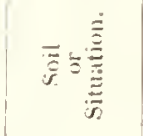 & $\begin{array}{ll} \\
0\end{array}$ & 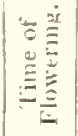 & 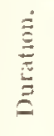 & 送 \\
\hline IIPERICUM. & ST. IOHN'S WO & $1 R T$. & & & & \\
\hline 1 calycinum & large-flowered.... & Thick. & yellow & $7-9$ & S. & 2017 \\
\hline 2 Androsivmum. & tutsan......... & Whoorls & yellow & 7,8 & S. & 1225 \\
\hline 3 quadrangulum & square... & Moi. niea. & yellow & 7,8 & P. & 350 \\
\hline 4 perforatum .. & perforated . & Bushy pl. & yellow & 7,8 & P. & 295 \\
\hline 5 dubium $\ldots$. & imperforated .. & M. thick. & yellow & 7,8 & $1 '$. & 296 \\
\hline 6 humifusum .. & trailing .... & Pastures & yellow & 7 & P. & 1226 \\
\hline 7 montanum .. & mountain . & Chal. so. & yellow & 7 & 1 . & 371 \\
\hline 8 barbatum .... & bearded ........ & Se. thick. & yellow & 9,10 & P. & 1986 \\
\hline 9 hirsutum .... & hairy & ('hal.ban. & yellow & 6,7 & P. & 1156 \\
\hline 10 pulchrum... & small upright .... & Woods & yellow & 7 & P. & 1227 \\
\hline 11 elodes ..... & $\operatorname{marsh} \ldots \ldots \ldots$ & Ap. Uogs & yellow & 7,8 & P. & 109 \\
\hline
\end{tabular}




\section{$82)$}

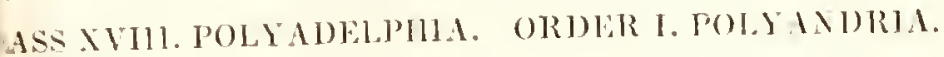

SPFCIFIC CIIIRACTER.

370. HYTHRICUM. Mypericiner. Juss.

Styles 5: fl. solitary : stem shrubby, branched, syuare : cal. segments blunt : 1. oblong.

Styles 3 : caps pulpy : stem slrubby, 4 -angular, compresserl.

Styles 3 : stem yuadrangular, herbaceous: L. witl pellucid dots: leat. of the calyx lanceolate.

Styles $3:$ stem 2 -edged : I. obtuse, with pellucid dots : leafl of the calyx lanceolate.

Strles 3: stem obsoletely quarhangular: L. obtuse, with few dots: leat. of the calyx elliptical.

Styles 2 : fi. somewhat cymove : stem 2-clged, prostrate : L. elliptical, smootl: leafl. of the calyx orate.

Styles 3: cal. with glandular serratures: stem erect, cylindrical, smooth: L. ovate, naked, clasping the stem.

Styles 3 : cal. and pet. fringed, and dotted : L. ovate, smooth, clasping the stem, dotted and glandular: stem erect, slightly angular.

Styles 3: cal, with glandular serratures: stem erect, cylindrical: L. ovate, pubescent.

- Styles 3: cal. with glandular serratures: stem cylindrical: 1.embracing the stem, cordate, smooth.

Styles 3: stem cylind. creeping, villous as well as the leaves, which are roundish: panicle few-flow: leafl. of the calyx obtuse, glandular. 



\section{CLASS XIX.}

SYNGENESIA. Compositer. Juss.

ORDER I. POLYGAMIA AQUALIS.

* Florets all ligulate.

OHyocirans. Receptucle chaffy. Pappus plumose. Cal. somewhat imbricated.

2 Cicnoriun. Reccpt. somewhat chaffy. Pappus chaffy, shortcr than the seeds. Cal. calycled.

9 Crepis. Recept. roughish. Pappus simple, mostly stipitate. Cal. calycled, scalcs deciduous, tumid.

8 Hieracium. Recept. nearly naked, dotted. Puppus simple, sessile. Cal. imbricater, ovate.

7 Apangla. Recept. naked, dotted. Pappus plumose, sessile. Cal. double; innermost imbricated.

2 Picris. Recept. naked. Pappus plumose. Seed transversely furrowed. Cal. double; innermost equal; outer lax.

1 Tragopogon. Recept. naked. Puppus stipitate, plumose. Cal. simple, many-leaved.

6 Leonrodox. Recept. naked. Pappus stipitate, simple. Cal. imbricated, double; scales of the outermost lax.

74 Lactuca. Recept, naked. Pappus stipitate, simplc. Cal. imbricated, cylindrical; margin membranous.

75 Prenantues. Recept.naked. Puppus ncarly sessilc, simple. Cal. calycled. Florets in a single row.

-3 Soncius. Recept. naked. Pappus simple, sessile. Cal. imbricated, ventricose.

31 Lapsana. Recept. naked. Pappus 0. Cal. calycled; inner leaflets equal, channelled.

* Florets all tubular, divaricating at the apex.

188 Carlina. Cal. ventricose; outer scales spinous; inner coloured, polished, radiant. Recept. chaffy. P'uppus plumose.

83 Arстіun. Cal. globular; scales spinous, hooked, inflexed,

85 Candurs. Cal. ventricose, inbrtcated; scales spinous. Recept. hairy. I'appas deciduous, capillary, rough.

26 Cxicus. Cal. ventricosc, imbricated; scales spinous, Recept. hairy. Pappus deciduous, plumose.

87 Oxopondum. Cal. ventricosc: scales spreading, spinous. Recept. honey-combed, somewhat chaffy,

:84 Serratula. Cul. nearly cylindrical, imbricaterl; scales not prickly.

Pajpus permanent.

Centaurea 1. 
*** Florets all tubular, erect, parallel, dense, flattish at top.

390 Luparonus. Recept. naked. I'appus rough. Cal.oblong, innbricated. Style semibifid, projecting.

391 Cunrsocons. Recept. nakerl. Papmus rougl. Cal. imbricated, hemispherical. Style scarcely longer than the florets. 389 Bidens. Recept. chaffy. P'uppus rough backwards. C'al. manyleaverl. Cor. sometimes radiated.

392 Diorss. Recept. chaffy. L'appus 0. Cal. imbricated, hemispherical. Florets with 2 spurs which border the seed. Tanacetum. Senecio 1. Aster 1. Anthemis 2.

\section{ORDER II. POLYGAMIA SUPERFLUA.}

* Discoid. Corollu of the marginal florets obsolete.

393 Tansceluu. Recept.naked. Seedscrowned. Cal. hemispherical, imbricated. Flarets of the radius 3 -cleft, obsolete, sometimes none.

396 Convza. Recept. naked. Pappus rough. Cal.imbricated, roundish. Florets of the radius 3-cleft.

395 Gxapralium. Recept. naked. Cal. imbricated; scales filmy, coloured. Flovets of the circumference awl-shaped. Pappus rough, or feathery.

394 Antemisia. Recept. naked or villous. P'apmes 0. Cul. imbricaterl, scales rounded, converging. Florets of the curcumference subulate, entire.

T'ussilugo 2, 3.

* Radiute. Florets of the radius ligulate.

405 Bells. Recept. naked, conical. Pappus 0. Cal. hemispherical, scales equal. Seerls obovate.

408 Matricaria. Recept. naked, almost cylindrical. Pappuso. Cul. Hattish, imbricated with scales, scariose at tho margin.

406 Cnnysantmimum. Recept. naked, rather convex. Papmuso. Cal. hemispherical, imbricited, with scales dilated, and membranous at the margin.

407 Promenunus. Recept. naked. Pappus marginate. Cal. lemispheical, imbricated, with sharpish scales scariose at the inirgin.

40.4 Dorosicun. Recept. naked. I'uppus simple. Seeds of the radius awntess. Cul. scales in a double order, equal, longer than the disk. 
02 Ixcis. Recept. naked. Papmus simple. Cal. imbricated. Cor. of the radius linear, very numerous. Anthere with 2. bristles at the base.

$\$ 7$ Frigenox. liccept. nakcd. Pappus simple. Cal. imbricated. Cor. of the radius linear, very narrow, numerous.

(1) Solnago. Recept. naked, pitted. P'uppus simple. Cal. imbricated with eonverging seales. Florets of the radius about 5 .

10 Aster. Recept. naked. Pappus simple. Cal. imbrieated, lowermost : cales spreading. Florets of the radius more than ten.

39 Sexerio. Recept. naked. Puppus simple. Cal. eylindrieal, many-leaved, equal, ealycled; scales dead at the tip.

38 Tussicago. Recept. natied. Pappus simple. Cal. simple, manyleaved, equal, somewhat membranous, ventricose at the base. Seeds obovate, eompressed.

03 Cineraria. Recept. naked. P'uppus simple. Cal. simple, manyleaved, equal, eylindrical. Seeds 4-angular.

99 Axтuenrs. Rerept. chafly. 'Pappus seareely marginate. Cal. hemispherical; seales nearly equal. Florets of the radius numerous, ablong.

10 Acuil.eA. Recept. ehaffy. Pappus 0. Cal. ovate, imbricated, unequal. Florets of the radius 5-10, roundish, or obeordate.

Bidens?.

\section{ORDER III. POLYGAMIA FRUSTRANEA.}

11 Ci:stanta. Recept. bristly. Pappus simple, or feathery; rarely wanting. Cor of the radius funnel-shaped, irregular, longer than those of the disk, without styles or stamens. 
CLASS XIA. SYNGENESIA. ORD. I. POLYGAMIA EQUALIS.

\section{$=$}

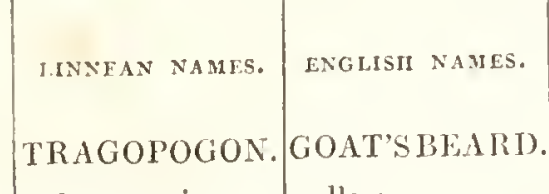

1 pratensis .... yellow........ Past. .. yellow

\begin{tabular}{|c|c|}
\hline 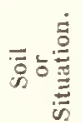 & 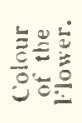 \\
\hline
\end{tabular}

2 porrifolius .. salsafy, or purple.

Moi. mea. purple

6 B.

434

B.

638

PICRIS.

\section{OX-TONGUE.}

1 echioides ....

bristly........

2 hieracioiles..

Bor of fi. ycllow

6,7

A.

972

Bor of fi. yellow

7,8 B.

196

LACTUCA.

SOW-THISTLF。

\section{SONCIIUS.}

1 caruleus - blue

Alp. pas.! blue

7,8

P.

2425

2 palustris ....

- Riv. ban. yellow

$7,8 \mathrm{P}$.

935

3 arvensis

Cora fi. yellow

8

1 .

674

4 oleraceus ....

Fields .. ycllow

$7-9$

1.

843

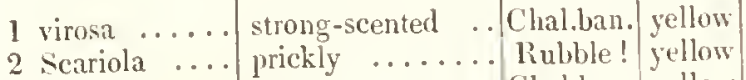

\section{LETTUCE.}

3 saligna...... least ........ Chal.ban. yellow

\section{PRENANTHES. WALL-LETT'TCE.}

1 muralis...... ivy-leaved ...... Chal. "ro. yellow

\section{LEONTODON.}

\section{1) ANUELION.}

1 'laraxacum ..

2 palustre $\ldots .$.

APARGIA. HAWKBI'.

1 hispida..... rough ......... Chil. pas. yellow 


\section{TLAGOPOGON.}

1 Calyx about equal to the corolla: L. entire, keeled, acuminate, dilated at the base: perlunc. cylind.

2 Cal. half as long again as the cor: L. entire, straight: pedunc. thicker upwards.

\section{PICRIS.}

1 L. waved; upper ones heart-shaped, clasping the stem: cal. outer of 5 broad prickly scales: pappus stipitate.

2 Stem elect, rough: L. lanceolate, wavy; radical ones toothed: Fl. corymbose: cal. lax: outer much shorter than the inner : pappus sessile: stem rough.

\section{SONCHUS.}

1 I'edunc, and cal. bristly, racemose: L. somewhat lyrate; terminal lobe large, deltoid.

2 Pedunc, and cal. bristly, somewhat umbellate: L. runcinate, sagittate at the base, rough in the margin.

3 Pedunc. and cal. bristly, somewhat umbellate: L. runcinate, denticulated, cordate at the base: ront creeping.

4 Pedunc. tomentous: calyx smooth: L. runcinate, toothed.

\section{LACTUCA.}

1 L. horizontal, denticulated, prickly on the kcel.

2 L. vertical, sinuated, denticulated, prickly on the kecl.

$3 \mathrm{~L}$. hastate-linear or pinnatifid, sessile, prickly on the keel.

\section{PRENANTHES.}

1 Florets five: L. runcinatc.

\section{LEONTODON.}

1 Uuter scales of thc calyx reflexed: L. runcinate, toothed, smooth.

2 Outer scales of the calyx shorter, erect, ovate: L. sinuated, toothed, almost smooth.

\section{APARGIA.}

\section{IEUYPNoIs, Eng. Bot.}

1 Stalks 1-flow: L. runcinatc, rough: florets hairy at their orificc, glandular at the apex. 
CLASS XIX. SYNGENESIA. ORD. I. POLYGAMIA LQUALIS.

\begin{tabular}{|c|c|c|c|c|c|c|}
\hline LINNEAY NAMLS. & FNGLISH NAMES. & 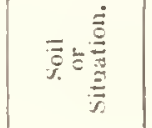 & 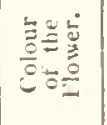 & 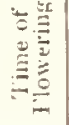 & $\stackrel{\dot{\bar{\Xi}}}{\stackrel{E}{E}}$ & 告 \\
\hline $\begin{array}{l}\text { APARGIA. } \\
2 \text { hirta....... }\end{array}$ & simple-haired .... & Grav. pa. & yellow & 7,8 & l'. & $* 555$ \\
\hline 3 Taraxaci .... & alpine ......... & Sc. alps & yellow & 8 & P. & $* 1109$ \\
\hline 3 autumnalis .. & autumnal .... & Mea. pas. & yellow & 8 & P. & *830 \\
\hline IIIERACIUM. & HAWKWEED. & & & & & \\
\hline $\begin{array}{lll}1 & \text { alpinum } & \ldots \\
2 & \text { Pilosella } & . .\end{array}$ & $\begin{array}{l}\text { alpine single flow. } \\
\text { mouse-ear ..... }\end{array}$ & $\begin{array}{l}\text { Alp. roc. } \\
\text { Dry past. }\end{array}$ & $\begin{array}{l}\text { yellow } \\
\text { yellow }\end{array}$ & $\stackrel{7}{5-7}$ & $\begin{array}{l}\mathrm{P} . \\
\mathrm{P} \text {. }\end{array}$ & $\begin{array}{l}\text { 11 } 10 \\
1093\end{array}$ \\
\hline 3 dubium $\quad \ldots$ & branch. mouse-ear & Hills!... & yellow & 7,8 & P. & 2332 \\
\hline 4 Auriculas.... & orange mouse-ear. & Mount. & yellow & 7 & P. & 2368 \\
\hline 5 aurantiacum... & great orange .... & sco. wo. & orange & 7 & P. & 1469 \\
\hline 6 murorum $\ldots$. & wall $\ldots \ldots \ldots$ & Rocks... & yellow & 6,7 & P. & 2082 \\
\hline 7 maculatum .. & stained-leaved.... & Wel. mou & ycllow & $6-8$ & P. & 2121 \\
\hline 8 sylvaticum .. & wood $\ldots . . . .$. & Chal. pl. & yellow & $6-8$ & P. & 2031 \\
\hline 9 pulmonarium & lungwort........... & Sc. roc. & yellow: & 7 & I'. & 2307 \\
\hline M0 Lawsoni .... & glaucous hairy .. & N.of liug. & yellow & 7 & P. & 2083 \\
\hline 11 paludosum .. & succory lca. mount & Wat.pl. & ycllow & $i$ & P. & 1094 \\
\hline 12 nolle $\ldots .$. & soft leaved ...... & Sc. wo. & yellow & 7,8 & $I^{\prime}$. & 2210 \\
\hline 13 cerinthoides .. & honcy-wort-leaved & Sic. roc. & yellow & 8 & P. & 2378 \\
\hline 14 villosum $\ldots$ & slraggy alpine ... & Alp.roc. & lem.col & 8 & P. & 2379 \\
\hline I5 IIalleri... & Ilallerian & sr. moll. & yellow & 7,8 & l. & \\
\hline
\end{tabular}




\section{Al'AliGIA.}

2 stalk, 1-flow. cal. smoothish, sealy at the base: I. lanceolate, tootherl, rough: seeels, outer row erowned with scales only.

3 Stalks nostly l-How, tumid and hairy at the summit: L. runcinate, smoutl : calyx hairy.

4 Stalks branehed: pedicels sealy: L. lanceolate, toothed or pinnatifid, nearly smootl.

\section{HIERACIUII.}

* Stalk radical, naked, 1-jlow.

1 L. oblong, entire, toothed: ealyx hairy.

2 L. elliptical, very entire, downy beneath: stolons ereeping.

* Stalk radical, naked, many-fow.

3 L. elliptic laneeolate, mostly very entire, hairy, rather glaucous beneath: stolons creeping: calyx bristly.

4 L. lanceolate, entire, liairy: stolons searcely as long as the leaves: calyx shaggy.

5 L. elliptieal, entire: stalk hairy: caly $x$ shaggy.

\section{*** Stem leafy.}

6 Stem corvmbose, 1-leaved: L. cordate-ovate, waved, toothed backwards, ehiely at the base.

7 Stem hollow, cymose, many-leaved: L. ovate-lanceolate, with teeth, pointing forward.

8 Stem simply racenose, solid: L. ovate-lanceolate, toothed, ehiefly at the base; teetl pointing forward.

9 Stem corymbose, solid, few-leaved: L. lanceolate, deeply toothed; teeth pointing forward.

10 Stem branched, few-leaverl: L. radieal stalked, elliptic-lanceolate, decurrent, glaueous, fringed, nearly entire.

11 Stem angular, hollow, smooth, eorymbose: L. cordate, embracing the stem, touthed, smonth: ealyx rough.

12 Stem angular, hollow, downy, corymbose: L. laneeolate, obsoletely toothed, hairy, embracing the stem; lower ones petioled.

13 stem eorymbose, solid: L. Lairy, somewhat toothed; upper ones ovate, half clasping the stem; radical ones elliptic-oblong, with shaggy fringed peduneles.

14 Stem leafy, hollow, shaggy : L. hairy, wavy, and toothed: cal. hairy: seeds angular.

15 stem erect, 1-2-flow. slichtly leafy: L. hairy; lower ones obovateoblong, stalked, toothed; upper lanceolate, smaller: eal. shaggy. 
CLASS XIX. SYNGENESHA. ORD. I. POLYGAMIA EQUALIS.

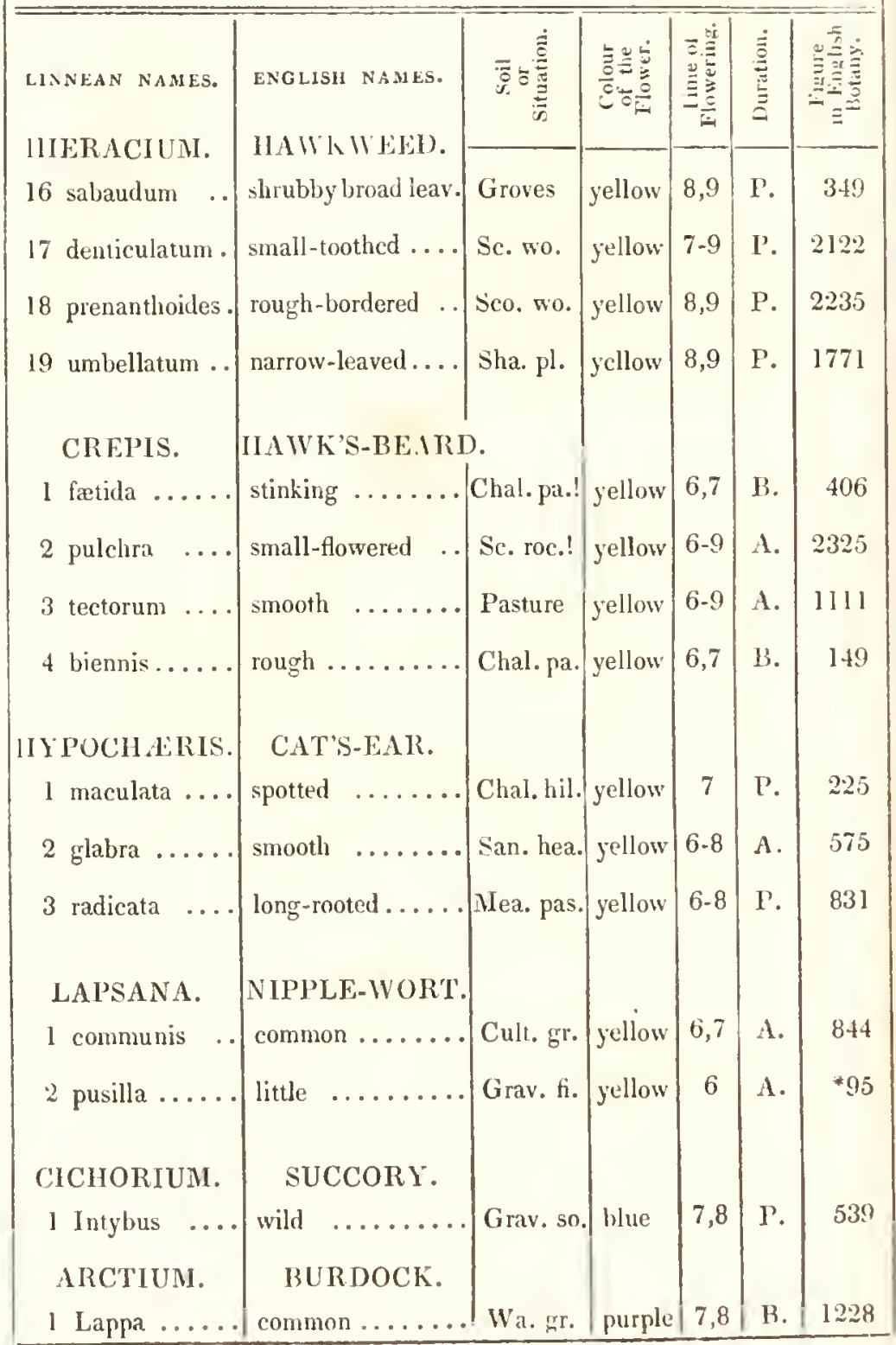




\section{HIERACIUN.}

16 Stem erect, many-flow: L. ovate-lanceolate, toothed, half embracing the stem, rough beneath.

17 Sten erect, solid, many-flow: L. sessile, elliptic-lanceolate, finely toothed, smootlish, glaucous beneath : pedunc. downy.

18 Stem erect, solid, many-flow: L. embracing the stem, somewhat toothed, rongh at the edge; upper ones cordate: pedunc. downy.

19 Stem erect, somewhat umbellatc: L. linear, slightly toothed, scattered : nearly smooth, as wcll as the calyx.

\section{CREPIS.}

1 L. runcinate-pinnatifid, hairy: petioles toothed: stem hairy: calyx tomentous.

$2 \mathrm{~L}$. downy, toothed; those on the stem somewhat arrow-shaped: stem panicled, corymbose: cal. pyramidal, snooth.

$3 \mathrm{~L}$. radical runcinate; those on the stem embracing, lanceolate, toothed: stem smooth: calyx rough: pappus sessile.

4 L. runcinate-pinnatifid, rough; forepart of the lobes toothed: calyx bristly, somewhat tomentous.

\section{HYPOCH ERIS.}

1 Stem solitary, nearly naked, mostly simple: L. ovate-oblong, entire, toothed.

2 Almost smooth: eal. oblong, imbricated: stems branched, somewhat leafy: L. dentate-sinuated: pappus of the marginal secds sessile.

3 L. runcinate, obtuse, rough: stems branched, naked, smooth: peduncles scaly: pappus stalked.

\section{LAPSANA.}

1 Cal, when infruit angular: stem panicled: L. ovatc, stalked, toothed: peduncles filiform.

2 Stemless: stalks subdivided, swelling and hollow at top: L. oboyate, rough-edged, toothed. Hyoseris minima. Eng. Bot.

\section{CICHORIUN.}

$1 \mathrm{Fl}$. in pairs; both ncarly sessile: L. runcinate.

\section{ARCTIUN1.}

1 L. cordatc, without prickles, petioled: cal. without cottony down. 
CLASS XIX. SYNGENLSIA. ORD. I. POLYGAMIA HQUMLIS.

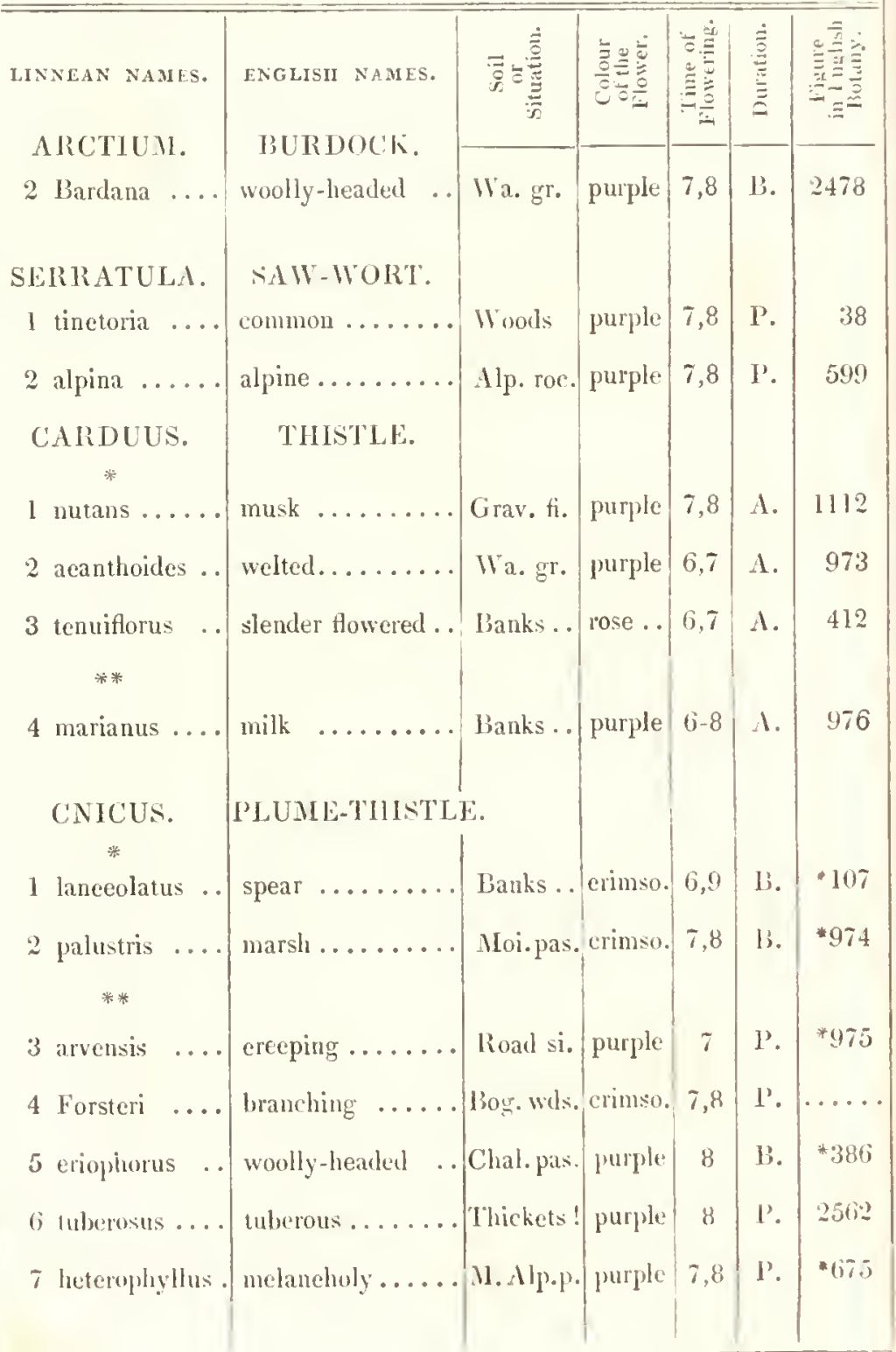




\section{$\triangle R C T I U M$.}

2 L. cordate, without prickles, pctioled, nearly cntire: cal. interwoven with cottony down.

\section{SERRATULA.}

1 L. seriated, fincly ciliated, lyrate-pinnatifid; terminal lobc largest: forets uniform: pappus roughish.

2 Cal. somewhat downy, ovate: L. undivided, woolly beneath: pappus plumose.

\section{CARDUUS. \\ Leaves decument.}

I L. interruptedly decurrent, spinous: flow. drooping: cal. seales lanceolate, sprearling upwards.

2 L. sinuated, spinous: cal. globular, somewhat peduncled: seales linear, recurved.

3 L. sinuaterl, spinous, tomentous: eal. almost eylindrieal, aggregate, sessile: scales lanceolate, somewhat reeurved.

\section{* Leures sessile.}

4 L. embraeing the stem, wavy, spinous ; radical ones pinnatifid: cal. seales leafy, ehannelled, bent baekward, spinous at tire margin.

\section{CNICUS.}

* Leaves decurrent, winging the stem.

1 L. pinnatifid, hispid; segments divarieated: (al. ovate, villous: $s t \mathrm{~cm}$ hairy, furrowed. Carduss. Eng. Bot.

2 L. pinnatifid, toothed, spinous, rough: Fl. aggregate: cal. ovate, clustered, with minute spines. Carduus. ling. Bot.

$$
\text { ** Leaves sessile. }
$$

3 L. pinnatifid, spinous : stem panicled: eal. ovatc, outer scalcs spinous : root creeping, tuberous. C'urduus. Eug. Bol.

4 L. slightly decurreut, pinuatifid, spinous; dlowny bencath : stenı panieled, hollow: ealyx ovate, rather cottony; outcr scales spinous.

$5 \mathrm{~L}$. bifarionsly pinnatifid, spinous, rough; alternate segments erect: eal. globose, villous. Curduus. Lng. Bot.

6 I. pinnatifid, loled, fringerl with priekles; lower oncs on long stalks : stem unarmed, grenerally l-flow: cal, minutely spinous.

$7 \mathrm{~L}$. embraeing the stem, laneeolatc, ciliated, entire or laeiniated, tomentous leneath: lil. mostly solitary, peduneulated. Curdurs. Eng. Bot. 
CLASS XIX. SYNGENESIA. ORD. I. POLYGAMIA RQUALIS.

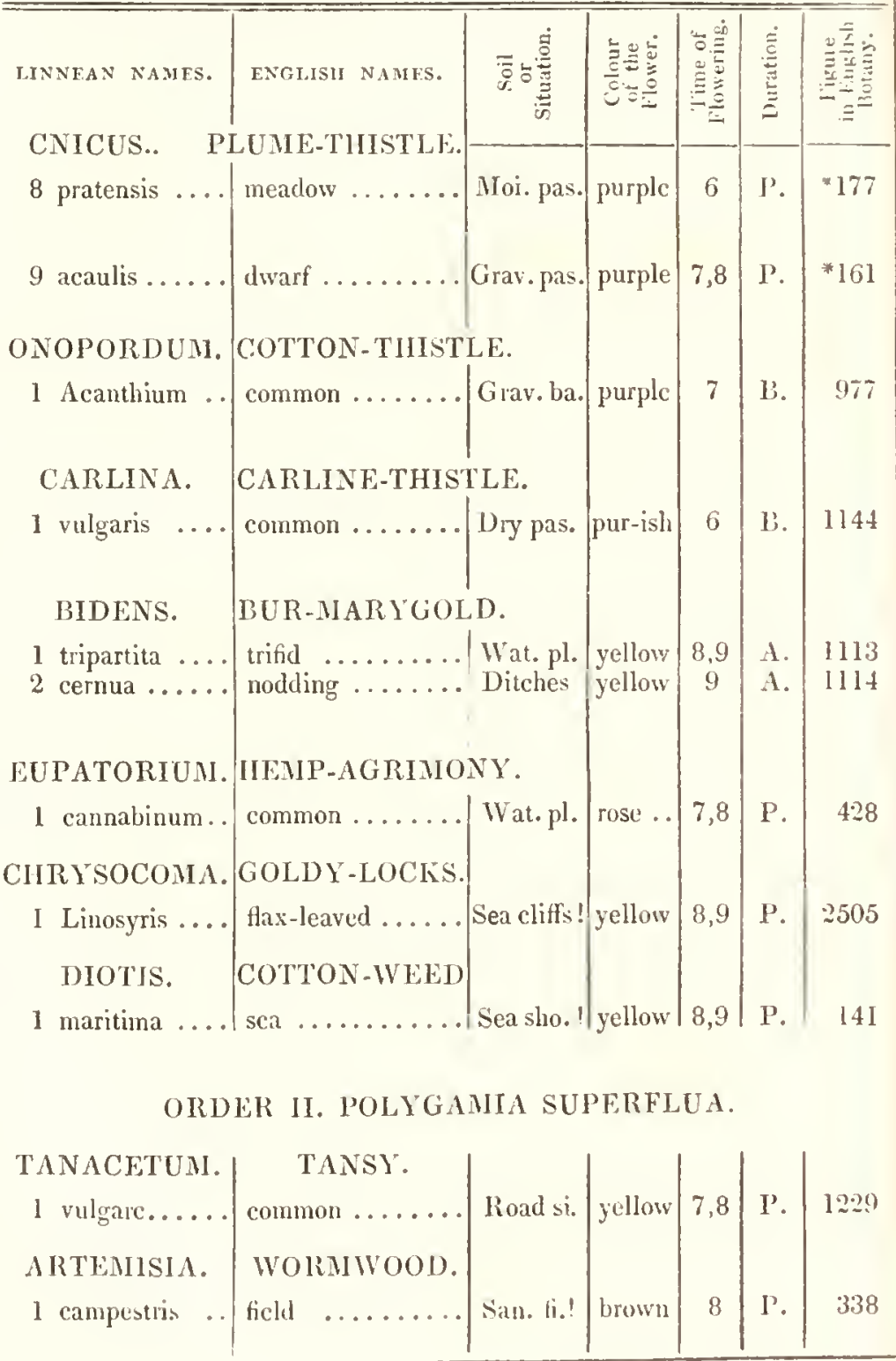




\section{CNICUS.}

8 L. lanceolate, wavy or lobed; ciliated, woully beneath: stcm ncarly naked, 1-How: calyx villous: seales tapering: inncr coloured. Carduns. Engr. Bot.

9 Stalks radical, 1-flow. shorter than the smooth calyx. Carduus. Eng. Bot.

\section{ONOPORDUM.}

1 L. ovate-oblong, sinuated, woolly on both sides: calyx-scales awlshaped, spreading.

\section{CARLINA.}

1 Stem many-flow. corymbose: Fl. terminal: outer calyx-scales pinnatifid; inner whitish.

\section{BIDENS.}

1 L. 3-cleft: bract. unequal: awns of the seeds 2 or 3 , erect.

2 L. lanccolate, serrated : Fl. drooping, bract. nearly equal, entire: awns of the seed about 4 , erect.

\section{EUPATORIUM.}

l L. 3-5-lobed; the middle lobe longest.

\section{CHR Y.NOCOMA.}

1 Herbaccous: L. linear, smooth: calyx seales loosely spreading.

$$
\text { 392. DIOTIS. }
$$

1 Pedune. corymbose: L. oblong, obtuse, erenated, very woolly.

\section{ORIER II. POLYGAMIA SUPERFLUA.}

\section{TANACETUM.}

1 L. bipinnatifid, decply serrated, naked.

\section{ARTEMISIA.}

1 L. many-eleft, lincar: stems procumbent before flowering, rod-like. 


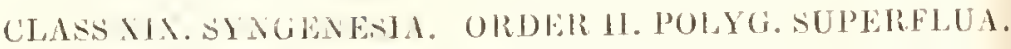

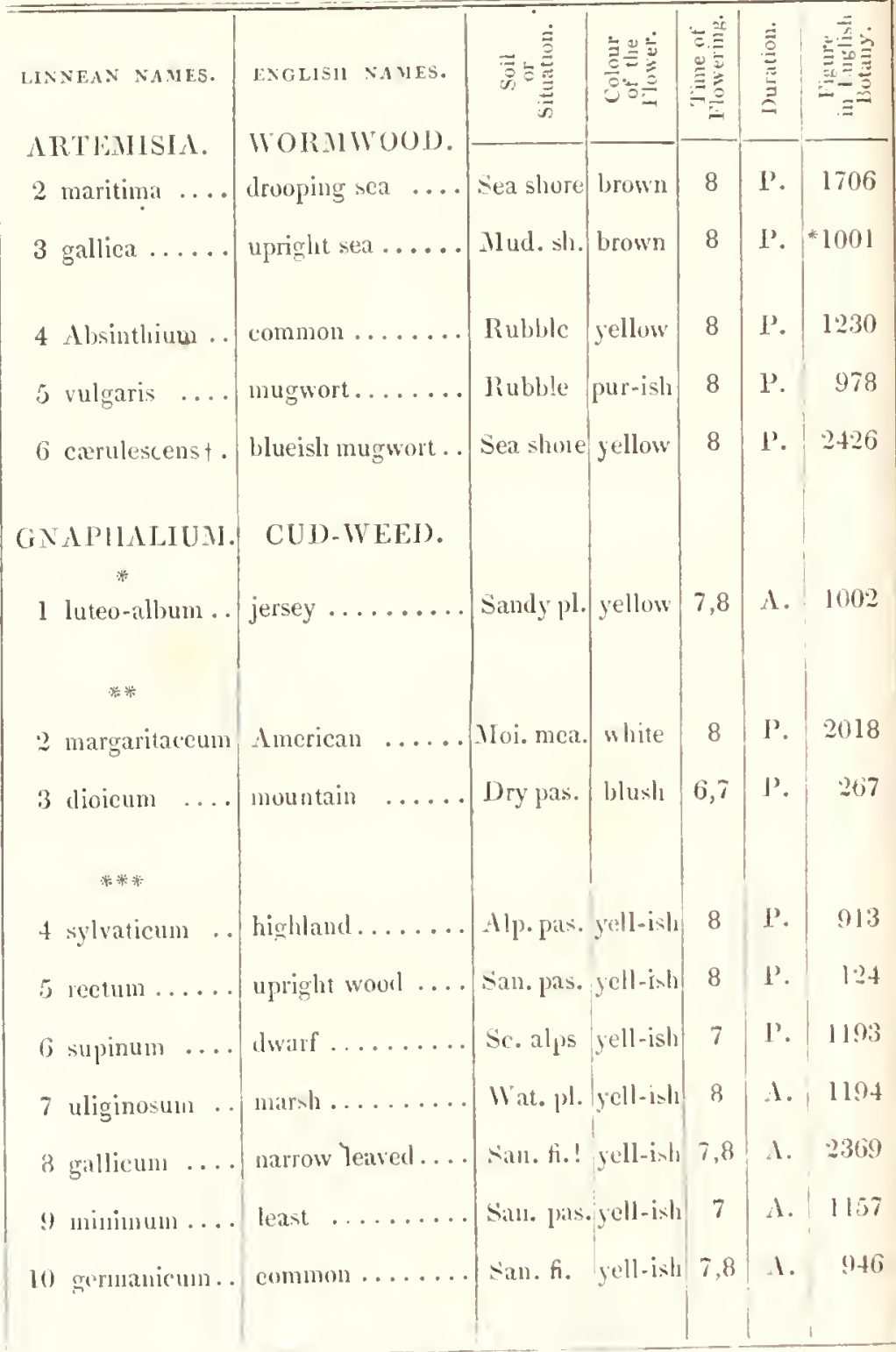




\section{ARTEMISIA.}

2 I. downy pinnatifid: the uppermost undivided: racemes drooping: reept. naked: l'l. oblong, sessile.

3 L. downy, pinnatifid t the radieal ones eapillary; the uppermost undivided : racemes erect: reeept. naked: FI. oblong. A. muritima. Fing. Bot.

4 L. many-parted, hoary with silky down: Fl. liemispherieal, pendulous : recept. hairy.

5 L. pinnatifid, Hat, eut, tomentous beneath : raeemes simple: Fl. ovate: reeept. naked.

6 L. hoary; those on the stem lanecolate, entire, tapering at the base; lowermost many-cleft: Fl. cylindrical : reeept. naked.

\section{GNAPHALIUM. \\ * Calyr vellow.}

1 Herbaceous: L. half-embraeing the stem, linear-oblong, waved, woolly on both sides; lowermost obtuse: Fl. elustered.

\section{Calyr white or redlish.}

2 L. linear lanceolate, acuminate, alternate, cottony: stem branehed above: corymb. fastigiate.

3 L. radieal spatulate, jointed, entire: shoots proeumb: stem very simple: corymb. simple, terminal: Fl. ilioeeious: pappus plumose, various.

$$
\text { Hats Calyz broun. }
$$

4 Stem simple, erect: Fl. spiked: L. lanceolate, narrower at the base, woolly on both sides.

5 Stem ereet : panicle many-flow: L. linear-lanceolate, almost naked on the uppersurface, silky beneath.

6 Stem decumb, very simple, racemose, few-flow: L. linear-lanceolate, tomentous on both sicles.

7 Stem much liranched, diffuse: Fl. crowded, teminating: L. linearlanceolate, tomentous.

8 Stem elect, branched: L. linear, levolute, acute: Fl. subulate, crowded, axillary.

9 Stem ereet, branched: I. lanecolate, aeute, flat: Fl. conieal, sonnewhat erowded, lateral and terminal.

I0 Stem ercet, proliferous: L. lanceolate: heads globose, many-flow, lateral and teminal: calyx-seales bristle-pointerl. 
CLASS XIX.SYNGENGAA. ORDER II, POLYG. SUPERELUA

LINNEAN NAMES.

CONY\%A.

1 squarrosa....

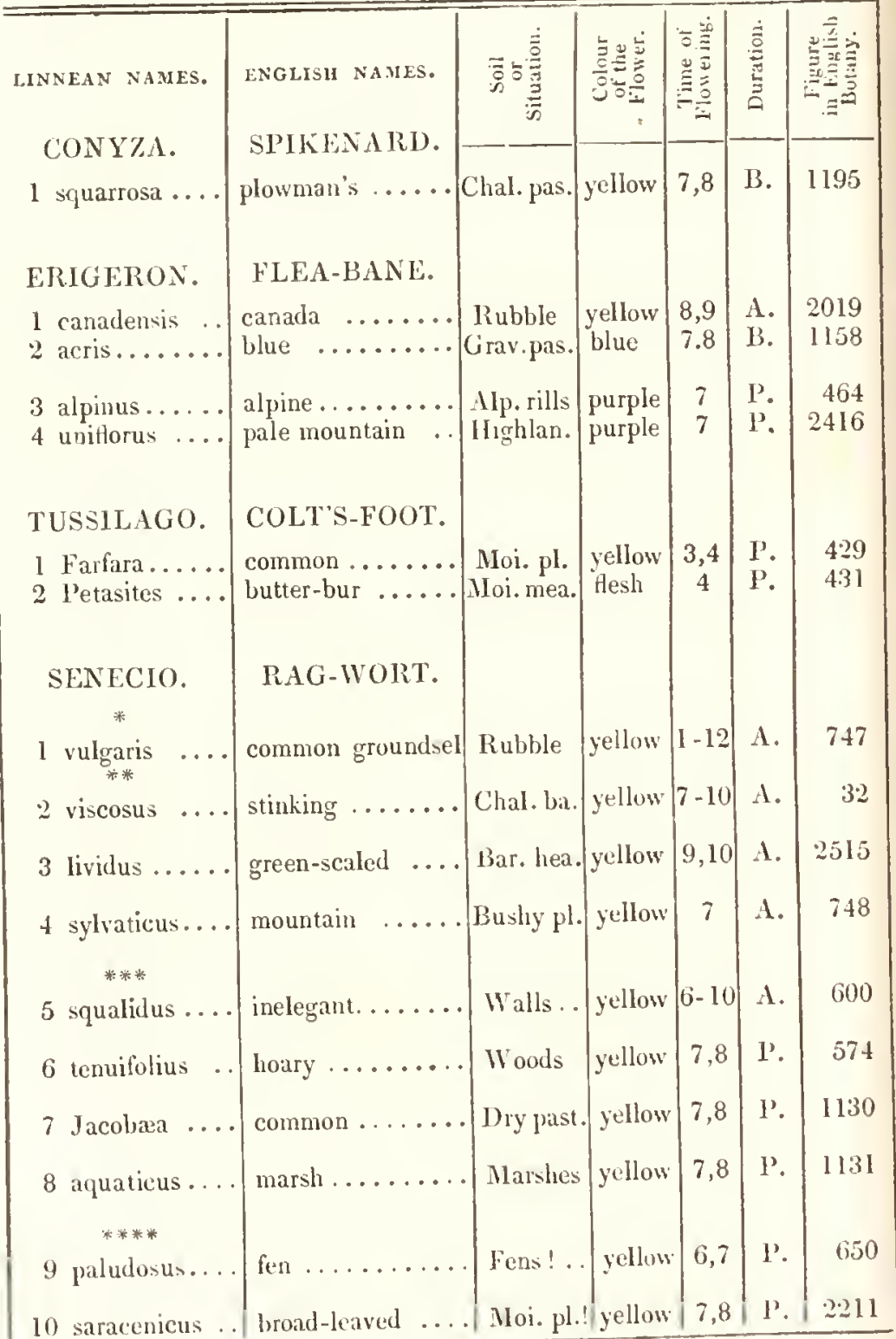




\section{SPECIFIC CIARACTER.}

\section{CONYZA.}

1 L. ovate-lanceolate, downy, crenate: stem herbaccous, corymbose: calyx-scales, leafy, rccurved.

\section{ERIGERON.}

1 Stem lairy, panicled, many-flow: L. lanceolate; lowermost toothed.

2 Stem raccmose: pedunc. mostly 1-flow: L. lanceolate, or tongueshaped, sessile.

3 Stems mostly 1 -flow : calyx rather hairy: flor. of the radius spreading. 4 Stems mostly 1-flow: cal. woolly: flor. of the radius erect, somewhat tubular.

\section{TUSSILAGO.}

1 Scape 1-flow, scaly: L. corlate, angular, toothed: Fl. radiant.

2 Panicle ovate: flor. almost all hermaph. with united anthers: L. cordate, unequally toothed; 3 -ribbed at the base.

\section{SENECIO. \\ * Flowers all fosculons.}

1 Fl. scattered: L. pinmate-sinuated, embracing the stem, toothed.

* Flouers radiate, radius constanily revolute.

2 L. pinnatifid, viscid: calyx-scales lax, almost as long as the inner: stem much branched, sprcading.

3 L. embracing the stem, lanceolate, pinnatifid, toothed: scales of the cal. short, with pale points.

4 I. sessile, pinuatifirl, lobed, toothed: calyx-scales sliort: stcm erect, straight, corymbose.

*** Fourers rudiute, rudius spreading: leares pinnatifid.

5 Rays elliptical, very entire: L. pinnatifid; segments somewhat linear, distant.

6 L. pinnatifid, somcwhat revolute, paler and pubescent loencath : stem erect, cottony.

7 I. Tyrate-bipimatificl, divaricated, toothed, smooth: stem erect: rays oblong, toothed: seeds of the disk silky.

8 Rays clliptical: 1. lyrate, serrated; lowermost obovate, entire: seeds smootl.

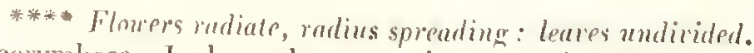

9 FI. corymbose: I. lanceolate, tapering, acutely serrated, somewhat villons beneath: stem straight, anfl hollow.

$10 \mathrm{Fl}$. corymlose: I. lanceolate, serrated, smoothish: stem solid. 
CLASS XIX.SYNGENESIA. ORDER II. POLYG.SUPERFLUA.

INNEAN NAMES.

ENGLISH NAMES.

$$
\text { 路莣 }
$$

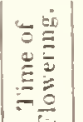

$\stackrel{\Xi}{\stackrel{\Xi}{\Xi}}$

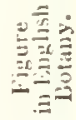

ASTER.

STAR-IVORT.

1 Tripolium

\section{INUL $\Lambda$.}

INULA,

1 Helenium .. elecampane......Moi.mea. yellow

7,8

P. 1546

2 dysenterica . common flea-bane. Wat.pl. yellow

P. $\quad 1115$

3 pulicaria .... small flea-bane .. Moi.hea. yellow

9 A.

1196

4 crithmoides .. samphire leaved .. Salt mar. yellow

\section{CINERARIA.}

FLEA-WORT.

1 palustris ....

2 integrifolia .. marsh Marshes

yellow

6,7

P.

151

l'.

DORONICUM. LEOPARD'S-BANE.

1 Pardalianches. great

M. pas.! yellow

5

P.

630

\section{BELLIS.}

DAISY.

1 perennis . common CIRYSANTHEMUM. OX-FYE.

1 Leucanthemum great white .....

2 segetum ... yellow corn.....

\section{PYRETHRUM. FEVERFEW.}

1 Parthenium . common ...... Rubble white 6,7 P. 
SPECIFIC CIIARACTER.

400. ASTER.

1 L. lanceolate, entire, fleshy, smooth, obscurely 3 -nerved : calyx-scales somewhat membranous, obtuse: fl. corymbose.

\section{I. SOLIDAGO.}

1 Stem somewhat zig-zag, angular: racemes panicled, erect, crowded: L. partly serrated.

\section{INULA.}

1 L. embracing the stem, ovate, wrinkled and downy beneath: calyxscales ovate, leafy.

2 L. embracing the stem, cordate oblong, downy: stem woolly, panicled : calyx-scales setaceous, hairy.

3 L. embracing the stem, undulated: stem much branched. hiairy : Fl. hemisplierical : radius very short.

4 L. linear, fleshy, generally 3 -pointed: calyx smooth.

\section{CINERARIA.}

1 Fl. cormbose: L. broad-lanceolate, toothed or sinuated : stcm villous leafy, hollow.

2 Stem erect. tcrminating in an imperfect umbel: bract. lisceolate: $\mathrm{L}$. shaggy: radical ones elliptical, obscurely toothed: the acst lanceolate.

\section{DORONICUNI.}

1 L. cordate, denticulated; radical ones petioled: thowe o:l the stem embracing.

\section{BELLIS.}

1 Scape naked, root creeping.

\section{CIIR YSANTHEMUM.}

1 L. embracing the stem, oblong. obtuse, cut, pinnatifid at the hase; radical ones obovate, petioled.

2 1. embracing the stem, glaucous, laciniated upwards, toothed at the base.

\section{PYRETIIRUM,}

I L. petioled, compound, fat: leaf. ovate, cut: pedune. branclued, corymlose: stem erect: rays shorter than the diameter of the dish. 
CLASS XIX.NYNGENESIA. ORDER II. POLYG.SUPTRFLUA.

\section{ENGLISII NAMES.}

2 inodorum....

3 maritimum .

I Chamomilla..

ANTHEMIS.

$\%$

1 maritima ...

2 nobilis

3 arvensis

+ Cotula eorn ......... sea

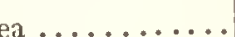
.

MAY-WEED.

wild ehamomile . .

Road si. white

5,7

A.

1232

\section{CHAMOMILE.} sea

\section{Sea coa.! white}

7

1.

2370

. eommon .......

Grav.pas. white

8,9

P.

980

eorn ............

1)ry fi.

- Corn fi.

stinking .......

**

5 tinetoria .

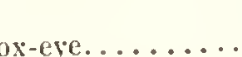

ICHILLTA.

1 Ptarmica....

2 serrata.....

3 Millefoliun ..

\section{IARROII.}

$$
\text { sneeze-wort..... }
$$

serrated

Moi.pl.

wlite

6,7

A.B?

602

l.

1772

4 tomentosa....

common

Pastuse

Se. pas. yellow 
PYRETIRUM.

2 L. sessile, pinnate, in many capillary segments: stem brancherl, spreading: crown of the seeds entire.

3 L. sessile, bipinnate, ficsly, awnless, convex above, keeled beneatls: crown of the seeds lobed: stems diffusc.

\section{MIATRICARIA.}

1 L. pinnate, smooth: leafl. linear, entire or laciniated: radius spreading: calyx-scales dilated, bluntish.

\section{ANTIIEIIIS. \\ * Radius white.}

1 L. bipinnatifil, acute, fleshy, somewhat hairy beneath : stem prostrate: calyx rather tomentons.

2 L. bipinnate-semicylind, acute, rather downy: scales of the receptacle scariose, scarcely the length of the florcts.

3 Recept. conical; scales lanceolatc, acute, keelcd, projecting; L. bipinnatifid, hairy: seekls crowned with a 4 -angular border.

4 Recept. conical; scales setaceous: seeds without any crown or border: L. bipinnatifid, slightly hairy.

$$
\text { * Radius yellow, like the disk. }
$$

5 L. bipinnatifid, serratcd, downy beneath : stem corymbosc, crect: seeds crowned with a membranous undivided border.

\section{ACHILLEA.}

1 L. lanceolate, acuminate, sharply serrated, smooth.

2 L. linear-lanceolate, sessile, downy, dceply-serrated, laciniated at the base: corymb. nearly simple.

3 L. bipinnatifid, laairy; segments linear, toothed, mucronated: stems furrowed.

4 L. bipinnatifid, woolly; scgments crowded, lincar, acute: corymb. repcatedly compound.

ORJER IIJ. POLYGAMIA FRUSTRANEA.

\section{CENTAURJA.}

\section{* Calux-scales jugrged or fringed.}

1 Calyx scales monbranous, laccrated: the lower ones pinnatifid: 1. linear-lanccolitte; raclical ones broader, toothed: Fl. radiant: pappus short, in a simple row. 
CLASS IIX. SYNGENESIA. ORD. II. POLYG. FRUSTRANEA.

\begin{tabular}{|c|c|c|c|c|c|c|}
\hline LINNEAN NAMES. & EXGLISH NAMIFS. & 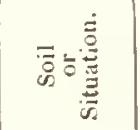 & 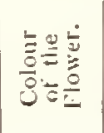 & 娣 & 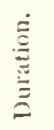 & 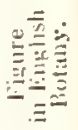 \\
\hline $\begin{array}{l}\text { CENT } A U R E A . \\
2 \text { nigra } \ldots . .\end{array}$ & $\begin{array}{l}\text { KNAPWEED. } \\
\text { black lesser...... }\end{array}$ & I'astures & purple & $6-8$ & P. & 278 \\
\hline 3 Cyanus .... & blue bottle ..... & Corn fi. & blue & 7,8 & A. & 277 \\
\hline 4 Scabiosa .... & greater ... & Corn fi. & purple & 7,8 & l'. & 56 \\
\hline 5 Isnardi...... & Jersey.......... & Jersey... & purple & 7,8 & I'. & 2256 \\
\hline 6 Calcitrapa & commonstar-thistle & Grav. so. & rose.. & 7,8 & A. & 125 \\
\hline 7 solstitialis.... & yellow star-thistle. & Fields! & yellow & 7,9 & $\Lambda$. & 243 \\
\hline
\end{tabular}




\section{CENTAUREA.}

2 Calyx-scales ovate, with capillary erect fringe: lower leaves lyrateangular; uppermost ovate: Fl. discoid: pappus short, tufted.

3 Calyx-scales serrated: L. linear, very entire; lowermost toothed towards the base.

4 Calyx-scales fringed, ovate: L. pinnatifid; segments lanceolate, a little hairy, somewhat toothed.

** Calyx-scales palmate-spinous.

5 L. lyrate-toothed, roughish, slightly embracing the stem: Fl. sessile, terminal.

*** Spines of the Calyx compound.

6 Fl. lateral, sessile: L. pinnatifid, toothed: stem divaricate-spreading, hairy.

7 Fl, solitary, terminal: L. decurrent, spineless, lanceolate; radical ones lyrate. 



\section{CLASS XX.}

\section{G Y N A N D R I A.}

ORDER 1. MONANDRIA. Urchidew. Juss.

* Anther of two distinct vertical cells, fired to the summit of the column. 112 Orсн1s. Lip or Nectury with a posterior spur.

413 Aceras. Nect. plane, without a spur. Cal. converging. $\$ 14$ Ophrys. Cal. spreading. Nect. convex, without a spur. 415 Herminum. Cal. spreading. Pet. 3-lobed. Nect. flat, without a spur.

*** Anther parullel to the stigma, of two cells, close together, permanent. 416 Goodrera. Cal. spreading, embracing the base of the nect. which is pouched, without a spur beneath, and undivided above. Column without wings. P'et. converging.

417 Nвотті. Cal. converging; embracing the base of the flat nect. which is without a spur. Pet. converging. Column without wings.

418 Listera. Cal.spreading. Nect. without a spur, nearly flat. Column without wings. Pet. spreading.

**** Anther terminal, inserted, permanent.

419 Epipactis. Vect. without a spur, tumid beneath, contracted in the middle, undivided at the end.

***** Anther a terminal deciduous lid.

420 MaLAX1s. Nect. without a spur, sessile, embracing the column with its concave base. Pet. spreading.

421 Corallonrhiza. Nect. unconnected with the column; spurred, or stalked at the base. P'et. spreading.

\section{ORDER IJ. DINNI)RIA.}

42.2 Crmupenum. Nect. ventricose, intlated. C'al. and l'et. spreading. Column with a terminal, dilated appendage.

ORDER III. HEXANDRLA.

423 Anisiolocina. Cal. of 1 leaf. Cur. 0. Stzg. 6-lobed. C'aps. 6-cclled. 
CLASS XX. GYNANDRIA.

ORDER 1. MONANDLIA.

\begin{tabular}{|c|c|c|c|c|c|c|}
\hline LINNEAN NAMES. & ENGLISH NAMES. & 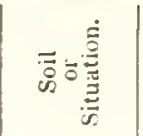 & 急哭言 & 它苞 & 寄 & 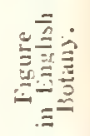 \\
\hline $\begin{array}{c}\text { ORCIIIS. } \\
*\end{array}$ & ORCHIS. & & & & & \\
\hline l bifolia ...... & butterfly........ & Woods & white & 6 & 1'. & 22 \\
\hline 2 pyramidalis .. & pyramidal ..... & Chal. so. & crimso. & 7 & l'. & 110 \\
\hline 3 Morio ...... & meadow ... & Mea. pas. & purple & 5,6 & P. & 2059 \\
\hline 4 mascula $\ldots$ & early purple .... & Shady pl. & purple & 4,5 & P. & 631 \\
\hline 5 ustulata ... & dwarf $\ldots . . . .$. & Chal. dow & purple & 6 & P. & 18 \\
\hline 6 fusca $\ldots .$. & brown .......... & Chal. hil. & bro-ish & 5 & 1. & ${ }^{*} 16$ \\
\hline
\end{tabular}

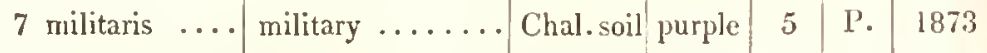

8 tephrosanthos. Monkey ........ Chal.soil! asl col. 5 P. .....

9 hircina ...... lizard ......... Chal.wo.! purple 7 P. ${ }^{*} 34$

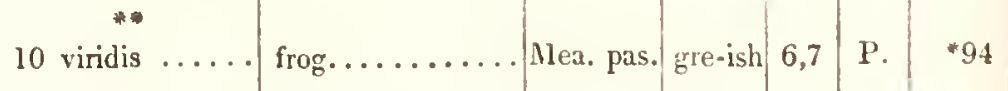

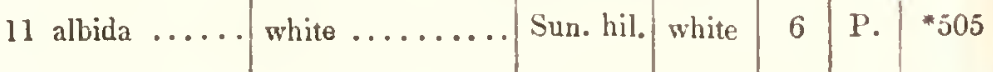

ACIIRAS. MAN-ORCHIS.

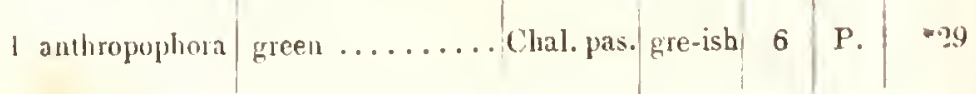

12 latifolia

marsh ......... Mloi. nuea. rose ..

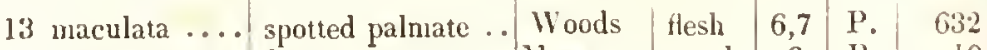

14 conopsea .... fragrant ........ Mlea. pas. purple 6 P. 10 


\section{SPECIFIC CIIARACTER.}

\section{ORCHIS.}

\section{* Bulbs undivided.}

1 Lip lanceolate, entire; half the length of its long spur: lateral calyxleaves spreading dowitward: bulbs oval, taper-pointed.

2 Lip of the nectary in three, equal, entire segments, 2 -horned above: spur elongated, filiform: bulbs oval.

3 Lip 4-cleft, crenate: spur obtuse, ascending: cal. convcrging, manynerved : bulbs oval.

4 Lip. 4-cleft, crenate: spur obtuse: calyx-leaves 3-ribbed; 2 lateral ones reflexed upwards: bulbs oval.

5 Lip 4-cleft, rough with points : spur obtuse, very short: cal. converging: L. lanceolate: bulbs oval.

6 Lip 5-cleft, rough; segments dilated: spur onc-third the length of the germen : cal. converging, obtuse: L. elliptic-oblong: bulbs oval. U. milituris, king. Bot.

7 Lip 5-cleft, rough: the middle lobes dilated, rounded: spur half the length of the germen: cal. converging, tapering: bulbs oval.

8 Lip 5-cleft: 4 segments equal, narrow, thread-shaped: spur obtuse; not half the length of the germen: cal. converging, tapering: bulbs oval. Bicheno, Trans. Jinn. Soc. aii.p. 33 .

9 Lip 3-cleft, downy; middle segment very long, lincar, twisted, emarginate: cal. converging: bulbs globose. Satyrium, Eng. Bot.

\section{* Bulls clustered, tapering.}

10 Bulbs clustered, divided: lip linear, 3-toothed; the middle tooth smallest: spur very short, emarginate. Satyrium, Eng. Bot.

11 Bulbs clustered in thrce pairs: lip 3-cleft: segments acute, middle one largest: spur one-third the length of the germen. Satyrium, Eng. Bot.

*** Bulbs pulmated.

12 Iips convex, 3-cleft. crenate: spul conical: brart. longcr than the flowers: stem hollow.

13 Lip 3-lolsed, crenate, flat: spur cylind. shorter than the germen.

$14 \mathrm{Lip}$ in 3 entire segments: spur slender. twice as long as the germen: cal, spreading.

413. ACERAS. Hort. Kew.

Opirrs, fing. Bot.

1 Lip longer than the germen. 
CLASs XX. GYYANDRIA.

OIiJER I. MUNANDliA.

\begin{tabular}{|c|c|c|c|c|c|c|}
\hline LINNEAN NAMES. & ENGLISH NAMES. & 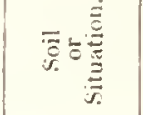 & $\begin{array}{l}\overrightarrow{8} \\
\dot{8}\end{array}$ & 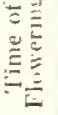 & 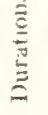 & $\frac{\sqrt{\Xi}}{\Xi}$ \\
\hline HERMINIUM. & MUSK-ORCHIS. & & & & & \\
\hline 1 monorchis .. & small ......... & Chal.ban. & gre-ish & 6,7 & $P^{3}$ & $* 7 I$ \\
\hline OPHRYS. & OPHRIS. & & & & & \\
\hline 1 muscifera.... & fy $\ldots \ldots \ldots$ & Chal. pas. & purple. & 6 & P. & 64 \\
\hline 2 apitera ..... & bee........ & Chal. pas. & purple & 7 & P. & 383 \\
\hline 3 arachnites... & late spider ..... & Clial. pas. & blush & 7 & P. & $\cdots$ \\
\hline 4 aranifera ... & spider ......... & Chal. soil & gretell & 4 & '?. & b5 \\
\hline 5 fucifera $\ldots$ & drone...$\ldots \ldots$ & Chal.hills & green & 5,6 & I'. & \\
\hline GOODYERA. & GOODYLIRA. & & & & & \\
\hline 1 repens ..... & creeping....... & Alp. wo.! & Hesll & 7 & P. & $* 289$ \\
\hline NEO'IIA. & LADI'S TRACE & & & & & \\
\hline 1 spiralis..... & swcet $\ldots \ldots \ldots \ldots$ & Mea. pas. & white & 8,9 & $I^{\prime}$. & $5+1$ \\
\hline 2 gemmiparal .. & proliterous ..... & Ir. coust & white & 7 & $I^{\prime}$. & \\
\hline J.ISTEliA. & $1 W \perp \amalg-131, \Lambda D L$. & & & & & \\
\hline 1 oviatal $\ldots .$. & common ....... & Giro. Meid. & green & 6 & $1 '$. & $* 1548$ \\
\hline$\because$ cordasts..... & heart-leaved .... & $\begin{array}{l}\text { Mi. liea. } \\
\text { ('lial. wo. }\end{array}$ & $\begin{array}{l}\text { greten } \\
\text { biomn }\end{array}$ & $\stackrel{7}{5,6}$ & $\begin{array}{l}\text { i". } \\
\text { l'. }\end{array}$ & $\begin{aligned} * 3.38 \\
* .43\end{aligned}$ \\
\hline 3 N DIIIIs & Dlias nest $\cdots . . \cdot$ & [ Ha. Whe. & 100 & & & \\
\hline
\end{tabular}


4I4. HFRMINIUII. Hort. Kew.

Opursa, Eingr. Bot.

1 J.. radical two, lanceolate.

\section{OPHRYS.}

I Lip 4-lobed, elongated, rather downy; disk polished: pet. linear: column obtuse.

2 Lip inflated, villous, 5-cleft; terminal segment awl-shaped, recurved: cal. coloured: column with a hooked point.

3 Lip longer than the calyx, dilated, tumid, 5-lobed; lobes inflexcd; terminal one flattened: cal. coloured: column with a hooked point: pet. deltoid, downy.

4 Lip the length of the ealyx, tumid, hairy, rounded, 4-lobcl: lobes refiexed: column aente, incurved: cells of the anther near together: pet. linear, smonth.

5 Lip longer than the calyx, obovate, hairy, undivided, with a spreading wavy margin: column bluntly pointed, incurved: pet. roughish, ovate at the base.

\section{GOODIER H. Hort. Kew.}

SAтrauru, Eng. Bot.

1 L. ovate: spike spiral: point of the nectary clongated, deflexed.

\section{NEOTTIA. Surarts.}

1 I. ovate, petioled: spike spiral, 1-rowed: lip ovate, entice: bract. downy, tumid. Ophrus. Fug. Bot.

2 L. lanccolate, as high as thic stalk: spike 3-ranked, spiral: bract. sinootl.

\section{JISTERA. Hort. For.}

$$
\text { OpIray, Eng. Bot. }
$$

1 L. clliptical, oppocite: nect. with 2 lincar-oblong, nearly parallel lobes: eolumn with a posterior hood.

2 L. heart-shaperl, opposite : ncct. 4-loherl.

3 Stem with sealy sheaths, witlout leaves: lip bifid; lolses spreading. 
CLASS XX. GYNANDRIA.

ORDER I. MONANDRIA.

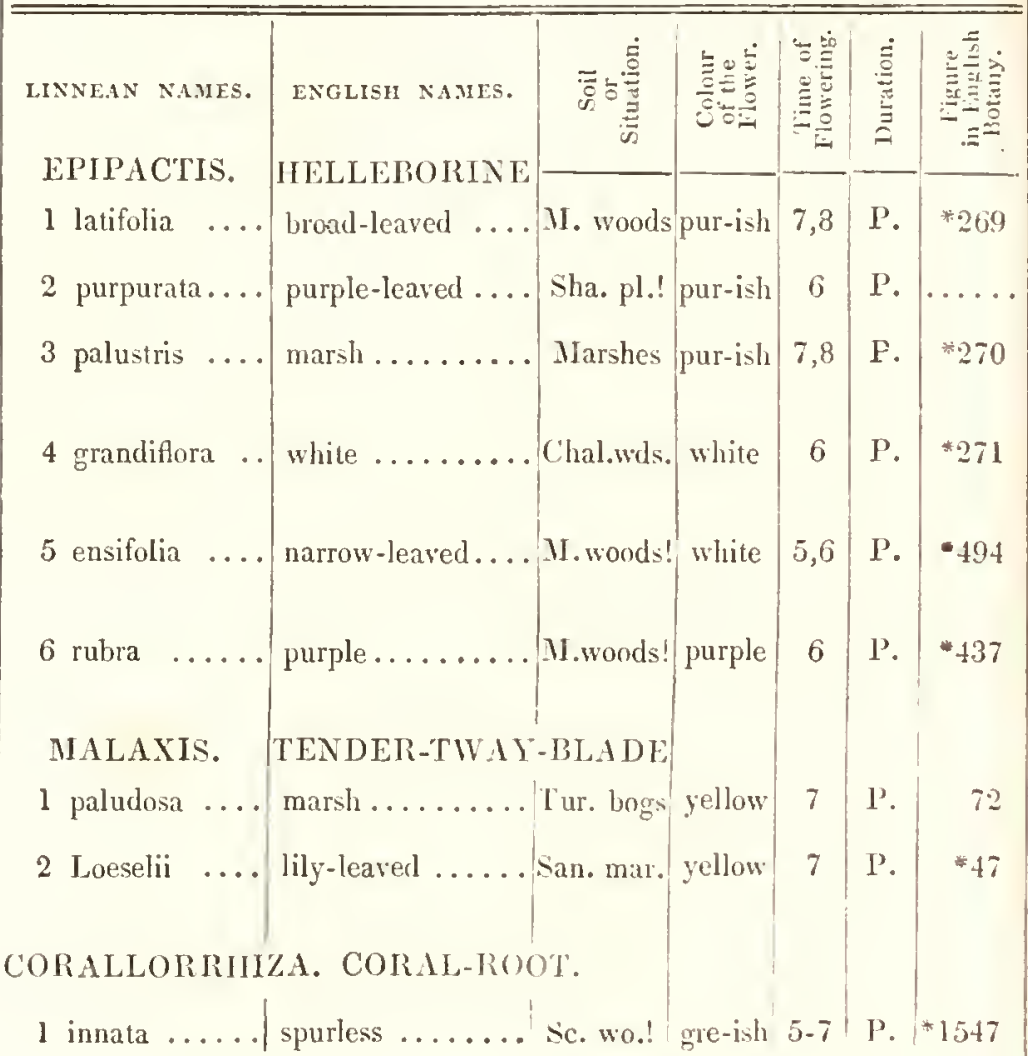

ORDER II. DIANDRLA.

\section{M}

2 Loeselii .... lily-leared ...... San
ORALLORRIILA. CORAL-RoOT.

1 innata ..... spurless ....... sc. wo.! gre-ish $5-7 \mid$ P. ${ }^{*} 1547$

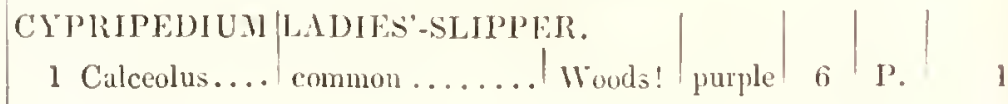

ORDER III. HEXANDRIA.

ARISTOLOCHA. BHRTH-WOHT.

1 Clematitis .. rommon ....... Woods vollow 7,8 . P. 
GPEITIC EIARAETER.

419. EPIIACTIS. Hort. Kew.

1 L. ovate, embracing the stem : Fl, drooping: lip very entirc, accuminate, shorter than the calyx. Serapias, Ling. Bot.

2 L. orate-lanccolate: bract. linear, twiee as long as the flowers: lip shorter than the calyx : germen downy.

L. laneeolate, embraeing the stem : Fl. drooping : lip crenate, obtuse, equal to the petals, with a notched protuberanee on the disk. Serapias, Eng. Bot.

H L. elliptic-lanceolate: bract. longer than the germen: Fl. sessile, ereet: lip obtuse, seareely equal to the calyx : with elevated lines on the disk. Serapius, Ling. Bot.

L. lanceolate: bract. much shorter than the germen: Fl. sessile, erect: lip obtuse, half as long as the calyx; with elevated lines on the di.k. Serupins, Eng. Bot.

6 I. laneeolate: bract. longer than the gcrmen: Fl. sessile, ereet: lip acute, marked with elevated undulating lines. Serapias, Ling. Bot.

\section{MAIAXIS.}

:I Stem 5-sided: L. about 4, spatulate, rough at the apex: Fl.reversed: lip coneare, aeute, entire, erect, half the length of the ealyx.

2 Stem 3-sided: L. 2, ovate-lanecolate: lip obovate, recurved, ehannelled, undivided, longer than the ealyx. Ophrys, Lng. Bot.

421. CORALLORRIIIZA. Haller.

Opurys, Eng. Bot.

11 Neet. spur short, not distinet from the slightly 3-lobed lip: root copiously branehed.

\section{ORDER II. DIANDRIA.}

422. CYPRIPEHIUM, Orchider. Juss.

I Stem leafy: lobe of the eolumn elliptieal, blunt, ehannelled: lip conpresced, shorter than the flat petals.

\section{ORDER III. IIEXANIORIA.}

\section{ARISTOLOCHIA. Aristolochive. Juss.}

1 L. eordate: stem erect: Fl. axillary, crowded, erect: cal. nnilateral. 


\section{CLASS XXI.}

\section{MONOECIA.}

\section{ORDER I. MONANDRJA.}

424 Eurmoran. . Involucrmm with numerous barren $y$. and one fertile. Bar. fl. Cul. 0. Cor. 0.

Fert. A. Cal.0. Cor. 0. Calps.3-lobed. Styles 3. cloven."

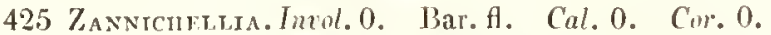

Fert. fl. C'al. 1-leaved. Cor. 0. Germ. 4, or more. seeds 4, stalked. Stig. peltate.

Callitriche. Chara? zostera? Typha minom.

\section{ORDER II. DIANDRIA}

\section{Carex.}

\section{ORDFR III. TRIANDRIA.}

427 Sparranium. Bar. H. Cal. 3-leaved. Corro.

Fert. A. Cal. 3-leaved. Con.0. Dinupe juiceless, 1-seeded.

428 Carex........ Bar. f. Catkin imbricated. Cal.glume I-valverl. Cor. 0.

Fert. H. Cutlin imbricated. Cal.glume l-valved. ('or. a hollow permaneut gitume, investing the loove seed.

429 Korresis.... (athin with opjosite seales imbricated in two rows.

Bar. H. Cul. inner scale. (or. 0.

Fert. H. Col. outer scale. (orro. Stig. 3. reedi I, naked.

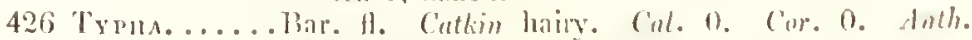
about 3. on each filament.

Fert. H. Cutkin hairy. Seed 1 on a hairy stalk. Amarouthens. 
.30 Littorell.... Yar. fl. Cal.4-leaved. Cor.4-cleft. Stam. capillary, very long.

Fert. f. Cal. 0. Cor. unequally 3-4-cleft. Style very long. $\therefore u l$. 1-celled.

33 Urtic........Bar. H. Cal. t-leaved. Cor. 0. Nect. central, cup-shaped. Stam. length of the calyx.

Fert. A. Cal.2.leaved. Cor.0. Seed 1, superior, polished.

32 Buxus.......Bar. 1l. Cal. 3-leaved. P'et.2. Rudiment of a gernien.

Fort. f. Cal.4-leaved. Pet.3. Styles 3. Caps. 3-beaked, 3-celled. Seeds 2.

31 Arxus. ......Bar. fl. Cal. scale of a catkin, permanent, 3-flow. Cor. 4-parted.

Fert. fl. Cal. scalc of a eatkin, permanent, 2-flow. Cor. 0. Stules 2. Seeds compressed, without wings.

Liviocalon. Myrica.

\section{ORDER V. PENTANDRIA.}

34 Хахтничм. . Bar. A. Cal. common imbricated; many-flow. with intermediate scales. Cor. monopetalous, funnclshaped, 5-cleft.

Fert. f. Cal.2-leaved, 2-flowered. Cor.0. Dinpe spurious, muricated, bifid. Nut. 2-celled.

35 Anarantinus. Bar. A. Cal. 3 or 5 -leaved. Cor. 0 . Stam. 3 or 5. Fert. ft. C'al. 3 or 5-leaved. Cor.0. Stules 2-3. Caps. 1 -celled, splitting all round. Seed 1.

35 Brionia. ...Bar. A. Cal.5-touthed. Cor. 5-cleft. Filum. 3. Anth. 5.

Fert. fl. Cal.5-toothed. Cor.5-cleft. Stule 3-cleft. Berry infcrior. Seeds few.

Fagus. Quercus. Atriplex.

\section{ORDER VI. IIEXANDRIA.}

37 Enrocavlon... Common cal. imbricated, many-flow.

Bar. Al, in the middlc. Cor. 4.6-parted. stam. 3.4-6.

Fcrt. Al. in the circumference. l'et.4-6. Stule I.

('aps. superior, 2-3-lobed. Seeds solitary.

Rumex. Quercus. 
439 Myrophllum. Bar. A. Cal.4-leaved. Pet. 4. Stam. 8. Fert. A. Cal.4-lcaved. Pet. 4. Stig. 4, scssilc. Seeds 4.

442 Porenum .... Bar.f. Cal.3-leaved. Cor.4-parted. Stam. 30 to 50. Fert. H. Cal.3-leaved. Cor.4-parted. Pist.1.2. Nut. 1-2-cclled, corticated.

440 Sagittaria. . Bar. A. Cal. 3-lcavcd. Pet. 3. Stam. about 24. Fert. H. Cal. 3-leaved. P'et.3. l'ist. numcrous. Seeds numerous, bordered.

438 Ceratopirleum. Bar. A. Cal.many-parted. Cor.0. Stum. 16-20.

Fert. A. Cal. many-partcd. Cor. 0. Stig. almost sessile. Drupa conipressed.

444 Farus. ...... Bar. A. in a cathin. Cal. in screral segments. Cor. 0. Slam. 5-20.

Fert. ค. Cal. double; outer inferior, prickly, 2.3flow. inner superior, 5-6-cleft. Cor. 0. Stules 5-6. Nuts 2-3. invested with the outer calyx.

443 Quercus. ... Bar. Al. in a cutkin. Cal. in several segments. Cur. 0. Slum. 8. ol morc.

Fert.fl. Cal. double; outer inferior, scaly, undivided; inner supcrior, 6-clcft. Cor. 1). Stule 1. Nut solitary, investcd, at its basc, with the outer calyx.

447 Corrues. ....Bar. A. in a rutkin. Cal. a 3-cleft scalc. C'or, 0. Stum. 8 or more.

Fert. fl. Cul.double; outer inferior, divided; inner superior, obsolctc. Cor.0. Styles2. Nut solitiry. bony, invested with the coriaceous outer calyx.

446 Carpinus..... Bar. H. in a cultin. Cal, a roundish scale. Cor. 0. Stam. 10 or more.

Fert. A. Cul. doublc; outer inferior, of scveral deciduous scalcs; immer supcrior, 3 -cleft. Cor. 0. Styles 2. Nut ovatc, angular, naked.

445 Betula ...... Bar. A. in a calkin. Cal. a temate scale. Cor. 0. Stam. 10-12.

Fert. A. in a cutkin. Cal. a peltate scalc, 3 -clcft, 3. How. Cor.0. Stules.2. Nut winged, deciduous.

441 Anux. ....... Common cal. a sheathing leaf, enclosing a common stalk, nakcd above. Cor. 0 .

Bar. fl. Silam. numcrous, in a dense ring, surmountel by another ring of abortive filaments.

Fcrt. fl. Germ. numerous, in a dense ring, below the stamcns. Styles 0. Stigr. dlowny. Berry with several seeds. 


\section{CLASS XXI. MUNUECIA. ORDER VIII. MONADELPHIA.}

H48 Prvus. Bar. fl. in a ratkin, naked. Stam. numcrous, on a common stalk.

Fert. fl, in a catkin of close, rigid 2-lipped, 2-flow. scales. Seeds 2. to each scalc, winged.

\section{Typha?}


GIASS XXI. MONOHC'1.

()R⿴囗十⺝R I. MONANI)RLA.

I.1.

I.INNEAN NAMES.

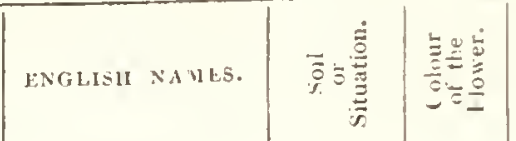

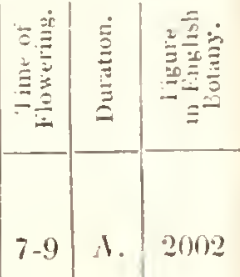

1 Peplis .....

purple.........

$* *$

2 leplus.....

petty

SPLTGL.

3 exigua ......

dwarf

C

('ul. g'r. yell-isli

7,8

A. 959

Sea coa. purple

959 Corn fi. tawny

1. $1: 336$

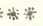

+ Iatlyris ....

capel

Dry st.pl! purple

5,6

B. 2255

* *米米

5 Portlandica .

\section{.}

6 paralia.....

7 helioscopia ..

8 stricta ..... warty

Gorn fi.! jellow

śca sh. jellow

Corn ti. yellow 8,9

A.

7,8

1. $\quad * 3: 3: 3$

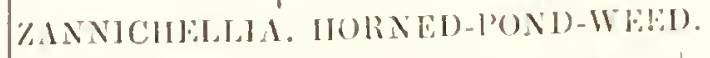

9 Esula ..... leafy-brancled .. Sha.wd-.! tawny

lo ('yparissias..

('yprcss .......

II IIiberna .... Go Thick yellow 6,7

$P$

I2 amygdaloirles. lrish $\ldots \ldots \ldots$

13 Chatacias... red sluubby .... Bus.M.pl puple 3,4 \begin{tabular}{l|l}
3,4 & P. \\
3,4 & $\mathrm{~S}$
\end{tabular}

1. 840

II (ls. Fi. pur-ith

$I^{3}$

1337 Mis. Giv yellow
Bus. M.pl pupple 256 $4+2$ I palustris .... common 
SlECIFLC CLARACIEL.

424. FUPHORBiA. Emphorbiaced. Juss.

* Dichotomous.

l I. entire, semi-corlate: involucrums solitary, axillary: stems procumbent: caps. smooth.

\section{*** Umbels trifid.}

2 Umb. trifid: dichotonous: bract. ovate: L. very entire, obovate, petioled: nect. lunate: seeds dotted.

3 UTmb. trifid : dichotonous: bract. lanceolate: L. linear: nect. horned seeds wrinkled.

*** Uimbels quedrifid.

4 Umb. 4-cleft, dichot: L. opposite, 4-ranked, sessile, entire, cordate at the basc.

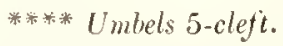

5 Umb. 5-cleft: dicliot: bract. nearly cordate, concave: L. hinearobovate, acute, smooth, spreading : nect. 4 : caps. rough-angled.

6 Unb. mostly 5-cleft, bifid: bract. cordate-miform: L. concave, imbricated upwards: neet. 5: caps. nearly smonth.

7 Umb. 5-cleft, trifid, dichot: bract. and L. obovate serrated: nect. 4. undivided : caps. smootl.

$8 \mathrm{Umb} .4$ or 5-cleft, trifid dichot: L. lanceolate, serrulatch : nect. 4. rounded, entire: caps. warty: seeds smooth.

\section{******** Unbel of 6 or more iranches.}

9 Bract. nearly cordate: L. uniform: nect. rhomboid, with 2 horns: caps. sinooth.

II0 Bract. somewhat cordate: L. of the stem, lanceolate: of the lateral branches, linear: nect. lunatc: caps. nearly smooth.

II Bract.ovate: L. obtuse: stem simple: nect. reniform, pointless: caps. warty, erect.

I2 Many axillary stalks bencath the umbels: bract. rounded, perfoliate: J. obtuse, hairy: caps. smooth.

II3 Nany axillary crowded stalks beneath the umbels: bract. pointed, pcrtoliate: L. lanceolate, downy: caps. hairy.

425. ZANNICIIELL1A. Fhuinles. Viar.

I Antl. 4-colled: stig. entire. 
CLASS XXI. MONOECIA.

ORDER III. TRIANDRIA.

$=$

LINNEAN NAMES.

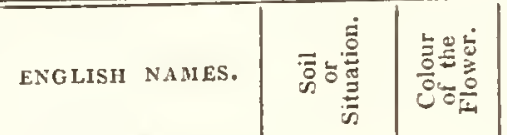

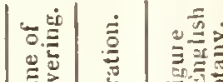
Lis

TYPHA.

1 latifolia

REED-MIACE.

2 angustifolia ...

great

Ditches

7

(n).......

Pools!..

3 minor ......

dwarf

Marshes!

$\begin{array}{llll}6,7 & \text { P. } & 1456\end{array}$

SPARGANIUII. BUR-REED.

1 ramosum ....

branched

Ditches

7,8 P.

744

2 simplex ....

unbranched......

3 natans ......

Stag. wa

7,8 P.

745

\section{CAREX.}

1 dioica

2 Davalliana

3 pulicaris ....

4 pauciflora....

few-flowered

***

6 curta

7 elongata ....

8 ovaiis ......

9 tcnclla ......

elongated oval spiked

slcnder-headed floating ........

\section{SEDGE.}

Fens

P. 543

5,6 l’. 2123

P. 1051

l. 2041

Bo.onll.!

$6 \mathrm{l}^{\prime}$.

204

(1)

white .........

Pool

Marshes
Marshes 


\section{TYPHA. Typhacer. Juss.}

1 L. somewhat convex beneath : catkin continuous: recept. hoary.

2 L. semicylindrical-flatlish, equal with the culm: bar. and fert. catkins a little distant: recept. scaly.

3 L. linear, convex beneath : catkins a little distant ; barren one leafy; fertile short and turgil; often interrupted: anth. nearly solitary: recept. naked.

\section{SPARGANIUM. Typhacer. Juss.}

1 L. 3 -sided at the base, their sides concave: common peduncle branched : stigma linear.

2 L. 3-sided at the base, their sides flat: common pedunc. simple: stig. linear.

3 L. floating, flat; concave at the base: common pedunc. simple: stig. ovate, very short: ball of bar. A. mostly solitary.

\section{CAREX. Cyperacer. Juss.}

\section{* Catkin single, simple.}

1 Catkin diocious: fruit ovate, nerved, ascending, serrulated at the margin : root creeping.

2 Catkin dioecious: fruit lanceolate-triangular, nerved, deflexed; angles scabrous at the apex: root tufted.

3 Catkin androg; the barren $\mathrm{fl}$. uppermost: fruit spreading, rcflexed, polished, tapering at both cnds: stig. 2 .

4 Catkin androg: fcw flow. the barren $f$. uppermost: fruit spreading, deflexed, awl-shaped: stigmas 3.

\section{** Spile compound, androyynous.}

5 Spikelets about 3 , rcmote: bar. $\mathrm{H}$. inferior, fruit divaricated, acuminate, entire at the mouth.

6 Spikelets about 6 , clliptical, rather remote, scarcely bracteated : glumes ovate, rathcr acutc, monbranous; about as long as the ovate, tumid, smooth fruit.

7 Spikelets numerous, oblong, rather distant, naked: fruit ovate-oblong, tapcring, bifid, recurved, many ribbed, longer than the glumes.

8 Spikelets about 6, oval, approximate, altcrnatc; with a bract. under the lowermost: Fr. lanceolate, rough-edged, striated, nearly entire; length of the scales.

9 Spikelets 3, bracteated, distant, minute, about 3-flow : Fr. clliptical, convex at each side: smooth, with a blunt, entire beak: stan. 2 . 
ORDER 111. TRIANDRIA.

\begin{tabular}{|c|c|c|c|c|c|c|}
\hline & & & & & & \\
\hline LINNEAN NAMES. & ENGLISH NAMES. & 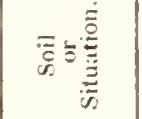 & 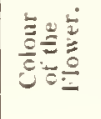 & 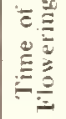 & 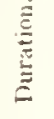 & $\begin{array}{l}0 \\
0\end{array}$ \\
\hline 10 remota...... & remote......... & Mlo.sh.pl. & & 5,6 & P. & 832 \\
\hline 11 axillaris & axillary clustered. & Bogs ! .. & & 5,6 & P. & 993 \\
\hline 12 incurva...... & curved......... & San.sh.! & & 7,8 & P. & 927 \\
\hline 13 arenaria & sea .......... & San. sh. & & 6 & P. & 928 \\
\hline 14 intermedia & soft brown ..... & Marshes & & 5,6 & P. & 2042 \\
\hline 15 divisa $\ldots$ & bracteated marsh. & Salt mar. & & 5,6 & P. & 1096 \\
\hline 16 muricata $\ldots$ & greater prickly .. & Moi pas. & & 5,6 & P. & 1097 \\
\hline 17 divulsa ..... & grey $\ldots .$. & Mio.sh.pl. & & 5 & P. & 629 \\
\hline 18 vulpina...... & great spiked $\ldots$. & Wat. pl. & & 5 & P. & 307 \\
\hline 19 teretiuscula . & lesser panicled & Bogs .. & & 5 & I'. & 1065 \\
\hline 20 paniculata & great panicled.... & Bog: . . & & 6 & P. & 1064 \\
\hline **** & & & & & & \\
\hline $2 !$ digitata...... & tingered .... & Woods! & & 5 & P. & 615 \\
\hline 22 claudestina .. & dwarf silvery .. & Sun. roc. & & 5 & I'. & 2124 \\
\hline$* * * *$ & & & & & & \\
\hline 23 pendulat & great pendulous & Mlot, wds. & & 5.7 & l'. & 2315 \\
\hline
\end{tabular}


SPEEIFIC CIIARAETER.

\section{CAREX.}

Spikelets solitary, remote, almost sessile: bract. very long, surmounting the eulm: lr. ovate, with a slightly eloven beak.

n spikelets screral, remotc, sessile: bract. elongated: Fr. ovate; its beak decply cloren.

2 Spikelets heaped into a head; the lower ones fertile: braet. membranous: culm roundish, smooth: L. channelled.

3 Spikclets crowded, spiked; upper ones eliiefly of barren, lower of fertile florets: bract. membranous; the lower ones leafy: eulm triangular: L. Hat : Fr, winged.

4 Spikelets erowded into a eommon spike; lower and upper ones fertilc; the intermediate barten: eulm triangular, crect.

5 Spike once or twiee compound: spikel. all androg: fertile florets inferior, the most numerous: braet. leafy, erect: fruit appressed : root creeping.

6 Spike oblong, prickly: Fr. divarieated, rough-edged, pointed, eloven : root fibrous.

7 Spike elongated, often branehing at the base; lower sprikel. remote: Fr.erect, smooth-edged, roughish at the eloven point of the beak: root fibrous.

8 Spike thrice compound, eompaet, obtuse: Fr. divarieated, with a notched, rough-edged beak: glumes pointed, angles of the eulm compressed, very sharp.

9 spike twice or thrice eompound, compact: Fr. spreading, gibbous; with a tapering, serrated beak: culm triangular, with eonvex interstiees.

20 spike thrice compound, branched, panicled, aeutc, interrupted: Fr. spreading, with an abrupt serrated beak: eulm sharply triangular, with flat interstiees.

***o* Bur. and jert. Jl. in separate cathins: bar. cutkin solitary: bruct. membranous. Stig. 3.

2l Bract. scarcely leafy, sheathing: spikes linear, lax, erect; barreu one shorter : fertile 2-3: L. flat.

22 Braet. scarcely leafy, sheathing: fertilc catkins remote, few flow. inclosed in the sheath: .. channellerl.

*** Bar. and fert. Jhr. in separate catkins: I harren catkin, rarely 2. bract. leat-like, and generally sheathing.

.23 Shearls nearly equal to the pedune: ferrile spikes cylind. very long, nodding: Fr. greatly crowded, ovate, beaked. 
CLASS XXI. MONOECIA.

ORDER 11I. TRIANDRA.

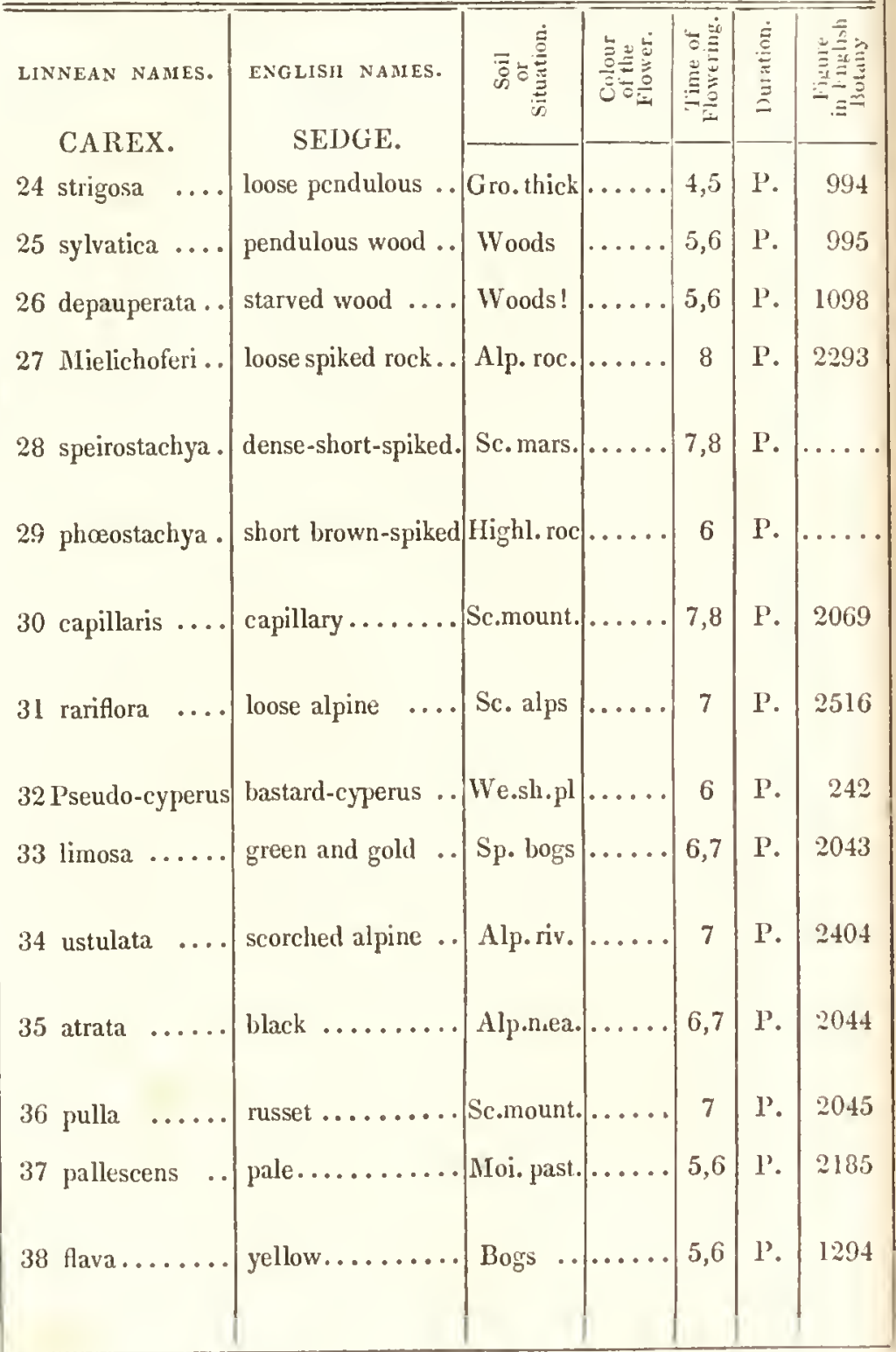




\section{$99)$}

SPECIFIC CHARACTER.

\section{CAREX.}

I4 Sheaths nearly equal to the pedunc: spikes filiform, lax, a little nodding: Fr. lanceolate, triangular, ribbed.

5 Sheaths not half the length of the pedunc: spikes filiform, ratlier loose, nodding: Fr. ovate, triangular, beaked, without ribs.

6 Sheaths much shorter than the pedunc: fertile spikes rcmote, erect, few-flow : Fr. inflated, ribbed, with a notched beak.

7 Sheaths not half the length of the pedunc: fertile spikes 3, distant, erect, lax: Fr. triangular, tumid, rough-edged : its beak cloven, membranous at the summit.

8 Sheaths shorter than the pedunc: fertile spikes about 3. distant, ovate, dense, many-How. crect: Fr. ovate, triangular, ribbed, smooth, with a deeply cloven beak, membranous at the orifice.

9 Sheaths shorter than the pedunc: fertile spikes 2 . distant, ovate, erect: Fr. ovate, triangular, smooth, with a cloven beak: scales of the barren spikes acute; of the fertile ones obtuse.

i0 Common sheath inuch shorter than the pedunc: fertile spikes ovate, lax, drooping: Fr. triangular, pointed, ribless, membranous at the tip : root fibrous.

1 Sheatlis scarcely any: fertile spikes few-flow. lax, drooping: Fr. obovate, bluntly triangular, slightly pointed, ribless: root creeping.

2 Sheaths scarcely any: fcrtile spikes cylind. pendulous, many-flow: Fr. spreading, furrowed, with a cloven beak.

33 Sheaths scarcely any : fertile spikes ovate, pendulous, many-flow : Fr. elliptical, compressed, ribbed, smooth-edged, without a beak: root creeping.

34 Shcaths scarcely any: fertile spikes ovate, pendulous: Fr. elliptical, smootl, compressed, beaked, with rough edges: root tufted, somewhat crecping.

35 Shcaths scarcely any : spikcs ovate, peduncled, pendulous; the terminal ore with many barren flowers bclow: Fr. elliptical, smooth, with a notclicd beak : stam. 2-3.

36 Sheaths none: fcrtile spikcs ovate; the lower one peduncled: Fr. elliptical, inflated, with a short emarginate bcak : stig. 2 .

37 Sheaths very short: fertile spikes cylind. pcdunclcd; when in fruit, pendulous: Fr. obovate, triangular, tumid, obtuse, with a minute abrupt beak.

38 Sheaths ncarly cqual to the pedunc: fertile spikes roundish: Fr. triangular, beaked, curved downwards: culm smoothish. 
CLASS XXI. MONOECIA.

ORDER 1H. TRIANALIA.

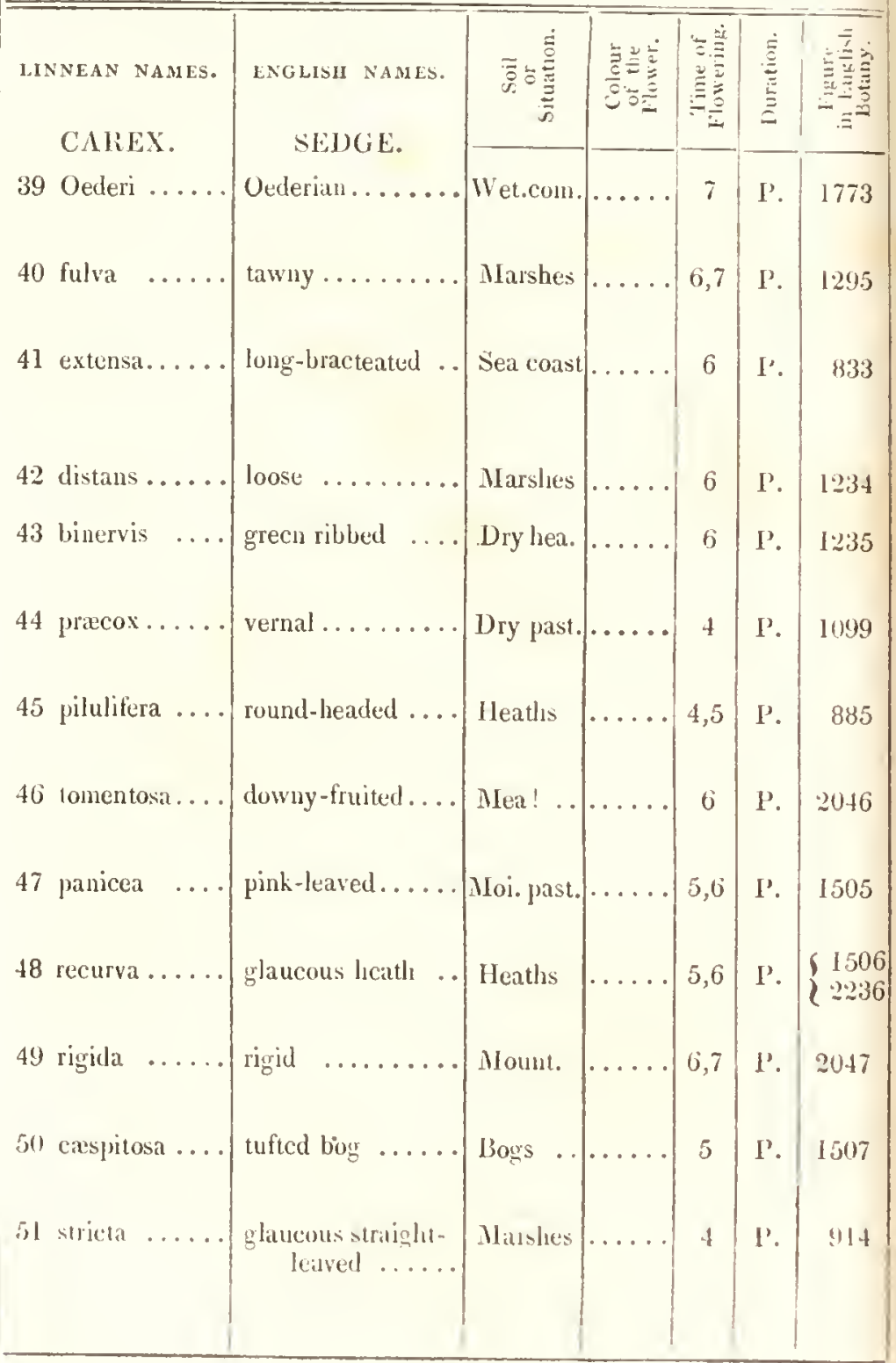


SPICIFIC CHARACTER.

\section{CARLS.}

Sheaths and pedunc, very short: fertile spikes roundich-ovate: lir. globose, triangular, dircet, smooth, with a straight, cloven beak : culm smootls.

Sheaths tubular, elongated, shorter than the pedunc: fertile spikes ovate: scales pointless: Fr. ovatc, triangular, smooth, with a straight, cloven, rongh-edged beak: culm rough.

Sheaths and pelunc. vely short: bract. long and sprcading: fertilc spikes elliptie-oblong, near together: seales somewhat awned: Fr. orate, triangular, with a short, smooth, cloven beak: eulm very smooth.

Sheaths tubular, elongated. nearly equal to the pedunc: fertile spikes elliptic-oblong, very remote: seales pointerl : culm smooth.

Sheaths tubular, tlongated, shorter than the pedunc: fertile spikes cylind. remote, partly compound, scales pointed: Fr. with 2 principal nerves.

4 Shenths nearly cqual to the pedune: spikes ovate, approximate: scales of the fertile ones pointed: Fr. pear-shaped, downy, with an abrupt, entire point.

i) Sheaths 0 : fertile spikes 2.3 sessile, crowded, roundish: seales pointed: Fr. triangular, roundish, downy, with a short, cloven beak.

Sheaths very short: fertile spikes 1-2, almost sessilc, cylind. obtuse: scales acutc: Fr. globose, slightly triangular, denscly tlowny, with a short, cluren beak.

7 Sheaths elongaterl, about half the length of the pedunc: fertile spikcs l-2, rather las, remote: $\mathrm{Fr}$. tumid, smooth, cloven at the summit, cuim smooth, obtusely triangular.

3 Shcaths short: fertile spikes 2-3, cylind. pendulous, on very long reeurved stalks: Fr.ellipical, triangular, roughish, obtuse, emarginate.

9 Sheaths 0 : fertile spikes ovate; the lowermost peluncled: bract. and L. lanceslate, reeurverl: Fr. triangular, somewhat compressed, with a short alorupt beak : stig. 2 .

0 Sheaths 0 : fertile spikes cylind. obtuse, crect, the lowermost rarely pcduncled: 1. and auricled braet. linear, erect: Fr. permanent, elliptical, flat, many-ribbed, with it slort, abrupt beak: stig. 2 .

1 Sheaths 0 : fertilc spikes nearly sessile, crcet, cylind. elongated, aeute: L. retieulated at the basc: Fr. elliptieal, flat, with a cloven beak; deciluous: stig. 2. 
CLASS XXI. MONOECIA.

ORDER III. TRIANDRIA.

\begin{tabular}{|c|c|c|c|c|c|c|}
\hline LINNEAN NAMES. & ENGLISII NAMES. & 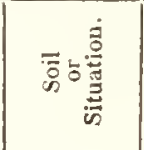 & 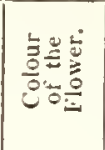 & 它䓪 & 莺 & 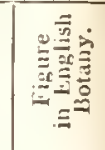 \\
\hline * ${ }^{*} * * * *$ & SEUUE. & & & & & \\
\hline 52 acuta $\ldots \ldots$ & slender-spiked .. & Wat.pl. & $\cdots \cdots$ & 5 & $\mathrm{P}$. & 580 \\
\hline 53 paludosa .... & lesser common ... & Wat. pl. & $\cdots \cdots$ & 5 & $\mathrm{P}$. & 807 \\
\hline 54 riparia $\ldots \ldots$ & great common.... & Riv. ban. & $\ldots \ldots$ & 4,5 & P. & 579 \\
\hline 55 læevigata .... & smooth stalk beak. & Bogs .. & $\ldots \ldots$ & 5 & P. & 1387 \\
\hline 56 vesicaria .... & short-spik'd bladder & Marshes & $\cdots \cdots$ & 5 & P. & 779 \\
\hline 57 ampullacea .. & slend. beaked blad. & Bogs .. & $\cdots \cdots$ & 5 & $P$ & 780 \\
\hline 58 hirta........ & hairy $\ldots \ldots \ldots$ & Wat.pl. & $\cdots \cdots$ & 5,6 & $P$ & 685 \\
\hline 59 secalina $\ldots$. & rye $\ldots \ldots \ldots . .$. & Sc.valleys & & $6,7 ?$ & P. & \\
\hline 60 stictocarpa .. & dotted ........ & Sc. IHighl. & $\ldots \ldots$ & $6,7 ?$ & P. & \\
\hline 6] angustifolia .. & narrow-leaved.... & Sc. mar. & $\cdots \cdots$ & $6 ?$ & P. & \\
\hline 62 fliformis $\ldots$. & slender-leaved.... & Bogs!... & & 6 & P. & 904 \\
\hline $\begin{array}{l}\text { KOBRESIA. } \\
\text { I caricina .... }\end{array}$ & $\begin{array}{l}\text { KOBRESIA. } \\
\text { sedgy } \ldots \ldots \ldots\end{array}$ & HI. alp.pl. & & 8 & P. & $* 1410$ \\
\hline \multicolumn{7}{|c|}{ ORDEIR IV. TETRANDRIA. } \\
\hline $\begin{array}{l}\text { LITTORELIAA. } \\
\text { I lacustris .... }\end{array}$ & $\begin{array}{l}\text { SHORE-IVEED. } \\
\text { plantain ........ }\end{array}$ & Wet.sa.p. & white & $0^{2}$ & $I^{\prime}$ & 468 \\
\hline
\end{tabular}


SPICIFIC CIIAIRACTER.

\section{CAREX.}

Bar. and fert. $f(0)$. in separate spikes. Barren spikes 2 or more.

Spikcs filiform: drooping in flower; erect, in fruit: Fr. clliptical, obtusc, with an undivided beak: stig. 2 .

Spikes cylind. rather blunt, ercet; fertile ones with taper-pointed scales: Fr. ovate, triangular, compressed, emarginate.

Spikes ercct: scales acuminate: Fr. ovate, tumid, with a deeply cloven beak.

Spikes cylind; fertilc ones peduncled: scales acuminate: sheaths very long: $\mathrm{F}$ i. triangular, with a cloven beak.

Fertile spikes cylind. slıort, abrupt, on short stalks: scales lanceolate, acute: sheaths 0 : Fr. ovate, inflated, with an elongated, cloven beak.

Fertile spikes cylind. elongated, almost sessile : scalcs lanceolate, acute: sheaths $0:$ Fr. globose, inflated, with a lincar, cloven beak.

8 Herbage hairy: fcrtile spikcs ovate, cylind. rcmote: scalcs awned: sheaths nearly cqual to the pedunc: Fr. hairy, tumid, with a deeply cloven beak: culm rough-edgcd.

9 Fertile spikes ovate-cylind. the lower one very remotc: scales acute: sheaths as long as the pedunc: Fr. ovate, compressed, roughedged, concave at the inncr sidc; with an elongated, linear, cloven bcak: culm smooth.

0 Fertile spikes 2, ovate, peduncled: scales pointed: sheatls scarcely any: Fr. obovate, obtuse, pointless, finely dottcd.

l Fertile spikes 1-2, ovate, peduncled: scales obtuse: sheaths $0: \mathrm{Fr}$. ovate, smooth, compressed, with a short, abrupt beak: L. linear, channellcd.

$; 2$ Fertile spikes ovatc: scales pointed: sheaths nearly equal to the pcdunc: Fr. ovate, hairy, with a deeply cloven bcak: L. linear, channelled, smooth.

429. KOBRESIA. Willd. Cyperacea. Juss.

1 Spikes aggregate, crowdcd, alternate. Schønus monoicus. Fng. Bot.

ORDER IV. TETRANDRIA.

430. LITTORELLA. Plantaginer. Juss.

1 L. lincar. 
CLASS XXII. MONOECI $\Lambda$.

ORDER II.TETRANDRIA.

\begin{tabular}{|c|c|c|c|c|c|c|}
\hline LINNEAN NAMES. & ENGLISII NA.HES. & ๘实言 & 昰 & 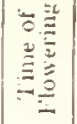 & $\stackrel{\stackrel{\Xi}{\Xi}}{\stackrel{\Xi}{\Xi}}$ & 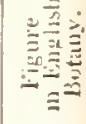 \\
\hline $\begin{array}{l}\text { ALNLS. } \\
\text { l glutinosus . }\end{array}$ & $\begin{array}{c}\text { ILDER. } \\
\text { eominolı ........ }\end{array}$ & Wat. pl. & $\ldots \ldots$ & 3 & 'l'. & * 1508 \\
\hline $\begin{array}{l}\text { BUTLX. } \\
\text { l sempervireus. }\end{array}$ & $\begin{array}{c}\text { BOX. } \\
\text { common } \ldots \ldots \ldots\end{array}$ & Clual. hil. & yellow & 4 & s. & 1341 \\
\hline URTIC'A. & NHTILI: & & & & & \\
\hline 1 pilulifera .... & Roman ...... & Rubbish & $\ldots \ldots$ & 6,7 & A. & 148 \\
\hline $\begin{array}{l}2 \text { urens } \ldots . . \\
3 \text { dioica } \ldots . . .\end{array}$ & $\begin{array}{l}\text { small } \ldots \ldots \ldots \ldots \\
\text { great } \ldots \ldots \ldots\end{array}$ & $\begin{array}{l}\text { Cult. gr. } \\
\text { Was gr. }\end{array}$ & & $\begin{array}{c}6-10 \\
7,8\end{array}$ & $\begin{array}{l}\text { A. } \\
1^{\prime} \text {. }\end{array}$ & $\begin{array}{l}1236 \\
1750\end{array}$ \\
\hline
\end{tabular}

\section{ORDFL I. PENTANDRIA.}

436

\section{XANTIIUN, BUR-U EED.}

1 strumarium ..

AMARANTHUS. XMARANTII.

1 Blitum......

BRYONIA.

wil

Dunghill!

BRTONY.

1 dioiea ...... red-berried ...... IIedges white

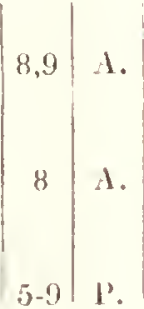

2544

$2: 212$

ORDIR VI. IIEXNDIIA.

BRIOC:AULON. I'HTWORT.

\begin{tabular}{l|l|l|l|l|l|l|l|}
1 septangulare. & jointed $\ldots . . . .$. & Ir.coa. & white & 8 & I'. & 733
\end{tabular} 
43I. ALNUS. Amentacer. Juss.

I L. roundish, somewhat wedge-shaped, waved, serrated, glutinous, downy at the branching of the veins beneatl. Betula Aluus. Eng. Bot.

432. BUXUS, Euphorbiacer. Juss.

1 L. ovate, eonvex, with footstalks downy at the edges: anth. ovatearrow-shaped.

433. URTICA. Urticer. Juss.

1 L. opposite, ovate, serrated, with transverse ribs: fertile flowers in globular heads.

2 L. opposite, elliptical, about 5-nerved: clusters nearly simple.

3 L. opposite, heart-shaped: elustered in pairs, very much branched, mostly diœecious: roots creeping.

\section{ORDER V. PENTANDRIA.}

434. Xanthiun. Composita. Juss.

1 Stem thornless: L. heart-shaped, 3-nerved at the base.

435. AMARANTHUS. Amaranthacer. Juss.

$1 \mathrm{Fl}$. in small lateral tufts, 3-cleft, triandrous : L. ovate: stem diffuse.

436. BRYONIA. Cucurbitaced. Juss.

1 L. palmate, rough on both sides with eallous points: Fl. dicecious.

ORDER VI. IEXANDRIA.

437. ERIOCAULON. Restiacer. R. Br.

1 Flor. 4-eleft: caps. 4-celled: stem with about 7 angles, many times taller than the channelled, taper-pointed leaves : Fl. convex : outer calyx-scales empty, rounded, smooth. 
CLASS XXI. MONOECIA. URULIR VH. POLYANDRIA.

438 CLEATOPHYLLUM. IIORNWORT.

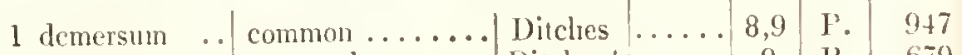

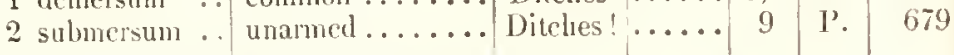

439 MYRIOPHYLIUM. WATER-MILFOHL.

1 spicatum .... spiked........ Ditches reddish 7,8 P. 83

2 verticillatum.. whorled ....... P'onds.. green 7 T'. 218

440. SAGITTARIA. ARROW-HEAD.

1 sagittifolia .. common ....... Rivers. white 7,8 P. 84

ARUA. CUCKOW-PINT

1 naculatum .. common ...... Shady pl. ..... 5 . 5 P. 1298

POTERIUM. GARDEN-IBURAET.

1 Sanguisorba.. common ...... Chal. hil. grcen 7 1'. 860 QUERCUS.

\section{OAli.}

1 Robur ...... commen british..

2 sessiliflora .. sessile fruited.... Woods

\section{FAGUS. BEECII ANI) CIHENUT.}

I Castanea .... Chesuut ........ Wroods .....

2 sylvatica .... common beech ..Chal. wd..... 4,5

886

T. $\quad 1846$

BFTUA.

BIRCII.

I alba.......

common ........... woods...... 4,5

dwitf ........... sic.mount..... 5

T. 2198

2 nаแก.......

I Betulus 
SPECIFIC CIARACTER.

438. CERATOPHYLLUM. Ceralophyller. DE C.

1 Fruit 3-spined: calyx-segments notched at the extremity.

2 Fruit spineless: calyx-segments acute, entire.

439. MIRIOPHYLUM. Halorager. R. Br.

1 Fl. in whorled, interrupted, leafless spikes.

2 Fl. all axillary.

440. SAGITTARIA. Alismaced. Juss.

1 L. arrow-shaped, acute.

\section{MRU11. Aroidere. Juss.}

1 Stemless: L. halberd-shaped, very entire: common stalk of the flowers, club-shaperl, obtuse.

\section{POTERIUM1. Rosacer. Juss.}

1 Thornless: stem somewhat angular.

\section{QUERCUS. Cupuliferu. Ricri.}

1 L. deciduous, oblong, broadest towards the end ; their sinuses rather acute; angles obtuse: pedunc. of the fruit elongated.

2 L. petioled, decidnous, oblong; their sinuses opposite, and rather acute: fruit scssilc.

\section{FAGUS. Cupnlifere. Ricu.}

1 I. lanceolate, sharply serrated, smooth bencath: prickles of the outer calyx compound, and cntangled : stig. 6.

$\therefore$ L. ovate, obtusely serratel: prickles of the outer calyx simple: stig. 3 .

445. BETULA, Imentacer, Juss.

11 L. ovate, acute, somcwhat deltoirl, unequally serrated, smoothish.

$\therefore$ L. orbicular, crenate, reticulated bencath.

\section{CARPINUS. Cupuliferie. Rль}

I Bract. of the fruit flat, oblong, serrated, with iwo lateral lobes: L. ovate, pointed, doubly serrated. 


\section{( 104$)$}

CLASS XXI. MONOECIA. ORDER VII. POLYANDRIA.

\begin{tabular}{|c|c|c|c|c|c|c|}
\hline LINNEAN NAMIS. & ENGIJSH NAMIS. & 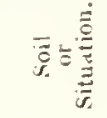 & 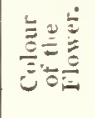 & 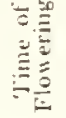 & 竎 & 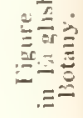 \\
\hline $\begin{array}{l}\text { CORILUS. } \\
\text { l Avellana... }\end{array}$ & $\begin{array}{c}\text { IHASEL. } \\
\text { common ..... }\end{array}$ & Woods & & & $\mathrm{T}$. & \\
\hline
\end{tabular}

ORDER VIII. MONADELPIIIA.

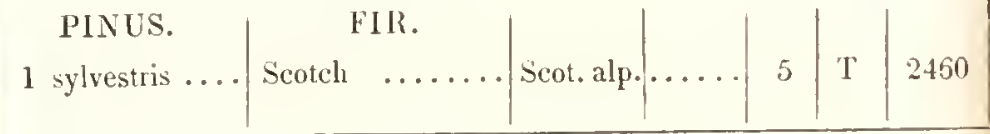


ICLASS XXI. MONOHCIA. ORDER VII. POLYANDIIA.

DPEIFIC CHARACTER.

447. CORYLUS. Cupuliferc. Ricu.

I Stip. ovate, obtuse: L. roundish, heart-shaped, pointed: young branches hairy: cal. shorter than the nect.

\section{ORDER VIII. MIONADELPHIA.}

448. PINUS. Conifere. Juss.

$1 \mathrm{~L}$. in pairs, rigid: cones when young stalked, recurved: crest of the anth. very small. 



\title{
CLASS XXII.
}

\author{
DIOECIA.
}

()RIER 1. MONANURLA

Several salices.

\section{ORDER II. DIANDRIA.}

149 S.atı........Barren Plant, Cathin imbricated. Cul. a scale. Cor.0. Nectary 1 or more glands at the base of the stamina. stum. I to 5 .

Fertile plant, Catkin imbricated. Cul. and Nect. as in the barren flowers. Cor. 0. Slig. 2. Cups. superior, l-celled, 2-valved. Seeds tufted.

\section{ORDER HI. TRIANDRI.1.}

1450 Enptrun ....Barren plant, Cal.3-parterl. Pet. 3. Stam. ea. pillary, 3 to 9 .

Fertile plant, Cal. 3-parted. Pet. 3. Stig. 9. Berry superior, 9-seederl.

451 Rusers ......Barren plant, Cul.6-leaved. Cor.0. Nect.ovate, tubular, learing the stamina within.

Fertile plant, C'al. 'Cor. and Nect. as in the barren flower. Stam. 0. Style 1. Berry superior, 3-celled. Seeds in pairs.

l'aleriana disica, and some salices.

\section{OLDER IV. TETRANDRIA.}

45) Ilypopuak.... Barren plant, Cal.2-parted. Cor. 0.

Fertile plant, Cal.tubular, 2-cleft. Style 1. Berry superior, 1-seeded. Seed ollong, with a double tunic. 
454 Mrrica . . . . Barren plant, Cal. scale of a catkin, concave. Cor. 0. Fertile plant, Cal. scale of a catkin, concave. Cor.0. Styles 2. Berry superior, 1 -seeded.

452 Viscum .......Barren plant, $\dot{C} a l .0$. P'et. 4. dilated and cohering at their base, resembling a calyx. Anth. sessile, fixed to the petals.

Fertile plant, Cal. submarginate. Pet.4. dilated at the basc. Style 0. Berry inferior, 1 -seeded. Rhommus 1. Enonymus 1. Urtica 3.

\section{ORDER V. PENTANDRIA.}

455 Hunulus ....Barren plant, Cal.5-leaved. Cor.3. Anth.with 2 pores at the apex.

Fertile plant, Cal.scale of a catkin, oblique, entire. Cor. 0. Styles 2. Seed 1, coated, winged with the calyx.

Ribes 4. Pimpinella 3. Bryonia 1. Salix 5, 19.

\section{ORDER VI. HEXANDRIA.}

456 Tauus ......Barren plant, C'al.0. Cor. 6-parted.

Fertile plant, Cal.6. Cor. 6-parted. Stule 3-cleft. Berry inferior, 3-celled. Seeds 2 together. Rumex $9,10$.

\section{ORDER VII. OCTANDRIA}

458 Rnodrola .... Barren plant, Cal. 4-parterl. Pet. 4. Nect. 4. notched.

Fertile plant, Cal. Pet. and Nect. the same. Pist. 1. Caps. 4. many-sceded.

457 Populus .... Barren plant, Cal. scale of a catkin, torn. Cor. turbinatc, oblique, entire.

Fertile plant, Cal. and Cor. the same. Stig. 4-8. Caps. superior, 1-celled, 2-valved. Seeds tufted.

\section{ORDER VIII. ENNEANDRIA.}

459 Mercuriazis. Barren plant, Cal. 3-parted. Cor. 0. Stam.9 to 12 . Anth. of 2 globose cells.

Fertile plant, Cal. and Cor. the same. Styles 2. Cups. 2-lobed, 2-celled. Sieds solitary. 
10 IHrdocharis. Barren plant, Cal.3-cleft. Pet. 3 . Filam. 3 of the interior beaked.

Fertile plant, Cal. and Pet. the samc. Siyles 6. Caps. 6-celled, many-sceded, inferior.

Einpetrum 1.

ORDER IX. DECANDRIA.

Silene 9. Lychnis 4.

ORDER X. ICOSANDRIA.

Rubus 14. Fragaria 2.

ORDER XI. POLYANDRIA.

Stratiotes 1. Populus 1, 3, 4.

\section{ORDER XII. MONADELPHIA.}

61 Iuniperus.... Barren plant, Cal. scales of a catkin. Cor. 0. Stam. 3.

Fertile plant, Cal. scales of a catkin, fewer, finally pulpy, united into a Berry with 3 Seeds.

462 Taxus .......Barren plant, Cal.0. Cor.0. Stam.numerous. Anth. peltate, lobed.

Fertile plant, Cal. cup-shaped, very entire. Cor. 0. Style 0. Seed 1, enclosed in the pulpy, unconnected calyx.

Salix 25, 26. 


\begin{tabular}{|c|c|c|c|c|c|c|}
\hline I,INEIX Numes. & EXGLISII NAMIES. & 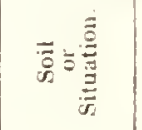 & 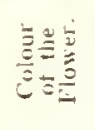 & 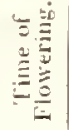 & 章 & $\begin{array}{l}0=0 \\
.\end{array}$ \\
\hline SALIIE. & WILLOW. & & & & & \\
\hline I triandia . & Iong-leaved..... & Hedges & & $5-8$ & $\mathrm{~T}$. & 1435 \\
\hline 2 Hoffmanniana. & short-leaved .. & Riv. side & & 5 & $\therefore$. & \\
\hline 3 laneeolata . & sharp-Ieaved .... & Meadows & & 4,5 & I. & 4536 \\
\hline \pm amygdalina .. & broad-leaved .... & Marshes & & 4,5 & s. & 1636 \\
\hline 5 pentandra... & sweet $\ldots . .$. & Riv. baus. & & 5,6 & $\mathrm{~T}$. & 1805 \\
\hline 6 nigricans .... & dark broad-leaved. & Fens Wds & & $t$ & $s$ & 1213 \\
\hline 7 phylicifolia .. & tea-leaved .. & Sc. Highr. & & 5 & s. & 1958 \\
\hline 8 liorreriana .. & dark upright ... & Se. Iligh. & & 5 & s. & \\
\hline 9 nitens $\ldots .$. & slining-leaved .. & Se.mou. & & 4 & S. & \\
\hline I0 Davalliana .. & Davallian ..... & sc. lligh. & & 5 & $\therefore$. & \\
\hline II Wulfeniana.. & Wulfenian ..... & Sevialleys & & 4,5 & s. & \\
\hline 12 tetrapla $\ldots$. & four-ranked...... & sc. High. & & 5 & s. & \\
\hline 13 bicolor ...... & slining dark-green & Woods & & $4, \tilde{5}$ & s. & 1806 \\
\hline It tenuifolia .... & thin-leaved ...... & liviv bill. & .. & 5,6 & s. & 2186 \\
\hline
\end{tabular}


449. SALIX. Amentacer. Juss.

* Adult leaves serrated, smoothish.

L. linear-oblong; rathcr unequally sloping at the base: stam. 3: germ. stalked, ovate, comprcssed, smooth : stig. nearly sessile. L. ovate-oblong; slightly rounded at the base: stam. 3 : germ. stalked, ovate, compressed, smooth: stig. nearly sessile.

L. lanceolate, tapering towards each end: petioles decurrent: germ. stalked, ovate, smooth: style as long as the stigmas.

L. ovate, rounded and unequal at the base: stam. 3: germ. ovate, compressed, smooth; its stalk almost as long as the scale: stig. nearly sessilc: young branches furrowed.

L. ovate, pointed, crenate, glandular: petioles glandular at the summit: stam. 5 or more, hairy at the base: germ. ovate, tapering, smooth, nearly sessile.

L. elliptic-lanceolate, acute, crenate, with a downy rib above; glaucous beneath: stam. 2, thrice the length of the liairy scales: germ. lanceolate, downy, on a short downy stalk.

7 L. elliptic-lanceolate, with wavy serratures; glaucous beneath : stip. glandular on the inner side: germ. lanceolate, stalked, silky: style twice the length of the stigmas: branches trailing.

8 L. lanceolate, with shallow, even serratures; glaucous bencath : stip. obsolete: branches upright: scales of the catkins acute, slaggy.

9 L. elliptical, acute, uncqually serrated; glaucous beneath, minutely downy, with a downy midrib above: stip. obsolete: branches spreading: catkins nearly sessile, with acute, shaggy scales.

0 L. obovate-lauceolatc, finely serrated, or minutely toothed, tapering at each end; rather glaucous beneath: pctioles midrib, and young branches downy: catkins with small rounded scales: caps. lanceolate, smooth.

1 L. obovate, or elliptical, somcwhat pointed, glaucous beneath: catkins dense, with liairy scales, longer than the stalks of the awl-shaped germens: style longcr than the stigmas.

2 L. elliptic-oblong, pointed; glaucous, with prominent veins, beneatls: stip. seini-cordate: scales shorter than the hairy stalks of the ovate-oblong, smooth germens: style as long as the stigmas.

3 L. elliptic-oblong, acute; glaucous beneatlı: jetioles dilated at the base : stip. pointed, serrated: scales obtuse, liairy; half as long as the densely downy, ovate, long-stalked germen.

14 I.. elliptical, acute, glaucous beneatli: stip. small or nonc: scales hairy: caps, ovate, smooth, on a short, smootl stalk. 
CLASS XXII. DIOECIA.

ORDLR II. DIANDRIA.

\begin{tabular}{|c|c|c|c|c|c|c|}
\hline LINNEAN NAMES. & ENGLISH NAMES. & 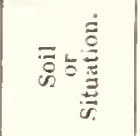 & 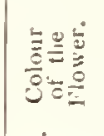 & 要 & $\stackrel{\dot{\bar{E}}}{\stackrel{\vec{E}}{E}}$ & $\begin{array}{l}0 \\
0\end{array}$ \\
\hline 15 malifolia .... & apple-leaved .... & Thiekets & & 4 & S. & 1617 \\
\hline 16 petiolaris .... & dark long-leaved.. & Marshes & & 4 & S. & 1147 \\
\hline 17 vitellina ... & yellow ......... & Marshes & ..... & 5 & $\mathrm{~T}$. & 1389 \\
\hline 18 decipiens .... & varnished & Woods & & 5 & T. & 1937 \\
\hline 19 fragilis ...... & erack .......... & Marshes & & 4,5 & $\mathrm{~T}$. & 1807 \\
\hline 20 Russelliana... & Bedford ........ & Wetwds. & & 4,5 & T. & 1808 \\
\hline 21 purpurea .... & bitter purple. & Low mea. & & 3 & S. & 1388 \\
\hline 22 Helix ..... & rose............ & Marshes & & 3,4 & T. & 1343 \\
\hline 23 Lambertiana. & Boyton ....... & Wet mea. & & 3,4 & T. & I 359 \\
\hline 24 Forbiana .... & basket osier..... & Osier tro. & & 4 & $\mathrm{~S}$. & 1344 \\
\hline 25 rubra $\ldots \ldots$ & green osier .. & Osier ho.! & & 4,5 & $\mathrm{~T}$. & 1145 \\
\hline 26 Croweana.... & Crowe's ..... & Wet mea. & & 4,5 & s. & 1146 \\
\hline 27 prunifolia.... & plum-leaved & Se. Iligh. & & 4,5 & S. & $\cdots \cdots$ \\
\hline 28 vacrinuifolia . . & bilberry-leaved & Sc. Iligh. & & 4 & S. & \\
\hline 29 verulosil & veiny-leaved & sc. Iligh. & & 4,5 & s. & 1362 \\
\hline
\end{tabular}




\section{SI'ECIFIC CHARACTER.}

\section{SALIS.}

L. elliptic-oblong, toothet, waved, thin, and craekling: stip. cordate, about equal to the petioles: scales obovate, bearded: germ. lanccolatc, smooth, on a short, smooth stalk.

L. lanceolate, glaucous beneath; somewhat unequal at the base: stip. lunate, toothed: catkins lax: scales hairy, shorter than the stalks of the orate, silky germens: stig. divided, sessile.

L. laneeolate, acute, with cartilaginous serratures; glaucous, and somewhat silky beneath: stip. minute, lanceolate, deciduous, smooth: gem. :csile, ovate-lanceolate, smootlı: scales linearlanceolate, acute, fringed at the base, longer than the pistil.

L. lanceolate, pointed ; floral ones partly obovate and recurved: petioles somewliat glandular: germ. tapering, stalked, smooth : style longer than the cloven stignas: branches smooth, highly polished.

L. ovate-lanceolate, pointed : petioles glandular: germ. ovatc, abrupt, nearly sessile, smooth: seales oblong, about equal to the stamens and pistils: stig. cloven, longer than the style.

0 L. lanceolate, tapering at each end : petioles glandular, or leafy: germ. tapering, stalked, longer than the scalcs: style as long as the stigmas.

I Decumbent: L. partly opposite; obovate-lanceolate; narrow at the base : stam. 1: stig. short, ovate, nearly sessile.

2 Erect: L. partly opposite, oblong-lanceolate, pointed: linear towards the base: stam. 1: style nearly as long as the linear divided stigmas.

3 Frect: L. partly opposite: obovate-laneeolate, pointed, rounded at the base: stip. 0: stam. 1: stig. ovate, obtuse, notched, short, nearly sessile.

4 Ereet: J.. alternate, with small stipulæ, lanceolate-oblong, rouncled at the base; glaucous beneatl: stam. 1: style nearly as long as the linear divided stigmas.

5 L. linear-lanceolate, elongater, acute; green on botlı sides: stan. combined bclow: stig, ovate, undivided.

6 L. elliptieal, glaucous beneath: stam. combined below.

$: 7$ L. broadly ovate, glaucous beneath: stem much brancherl: caps. ovate, Nhagoy, like tlıe scales, with silky hairs.

!8 Decumbent: 1. lanceolate-ovate, glaucous and silky beneath: eaps. ovate, silky.

29 Erect: 1. ovate; reticulated with prominent veins above; glaucous beneath: caps. ovate, silky. 


\begin{tabular}{|c|c|c|c|c|c|c|}
\hline LINNEAN NAMES. & ENGLISH NAMES. & 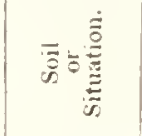 & 送 & 它总 & 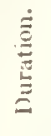 & 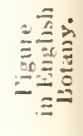 \\
\hline 30 myrsinites.... & green whor tle-leav. & Sc. High. & & 5,6 & s. & 1360 \\
\hline 31 Dicksoniana.. & $\begin{array}{l}\text { broad leaved moun- } \\
\text { tain }\end{array}$ & Sc. High. & & 4 & S. & 1390 \\
\hline 32 carinata & keeled-leaved .... & Sc. Ilight. & & 4 & S. & 1363 \\
\hline 33 Arbuscula & little tree........ & Sc. High. & & 4 & S. & 1366 \\
\hline 34 livida .. & livid dwarf ... & Sc. Low.! & & . . & s. & \\
\hline 35 herbacea .... & least $\ldots . . .$. & Mount. & & 6 & $s$. & 1907 \\
\hline 36 reticulata .... & wrinkled ........ & Mount. & & 6 & S. & 1908 \\
\hline *米楼 & & & & & & \\
\hline 37 glauca ... & glaucous........ & Sc. Ifigh. & & 5 & S. & 1810 \\
\hline 38 Stuartiana . . & small-leav. shitggy & Sc. High. & & 7,8 & S. & 2586 \\
\hline 39 arenaria & downy mountain.. & se. moul1. & & 5,6 & $\therefore$. & 1809 \\
\hline 40 lanila $\ldots .$. & woolly broad-leav. & sc. lligh. & & ... & s. & \\
\hline 41 argentea .... & silky sand $\ldots .$. & Sea sli. & & 5 & s. & 1364 \\
\hline 42 fortida. & fislly $\ldots . . .$. & Moi. liea. & & 5 & S. & 1962 \\
\hline 43 repens. & common dwarf. & San. licit. & & ij & $\therefore$ & $18: 3$ \\
\hline
\end{tabular}


WIIIFIC CHARACTER.

\section{SA]AX.}

Foung branches hairy: L. elliptical, veiny, polished on both sides: germ. stalked, downy: caps. awl-shaped.

L. clliptical, acute, slightly toothed, glaucous bencath : cathins ovate, short, crect: gcrm. stalked, ovate, silky: stig. nearly sessile.

L. orite, finely tootled, minutely veined, foldcd into a kcel: cathins cylind. with rounded, hairy scales: germ. sessile, ovate, silky.

Branches downy: L. lanccolatc, acute, obscurely tootherl, ylaucous beneatl, silky when young: catkins ovate, erect : germ. stalked, ovate-lanceolate, silky.

L. elliptic-oblong, obscurely toothed, livid beneath : stip. 0 : germ. nearly cylint. downy; its stalk twice as long as the scale: stig. nearly sessile.

L. orbicular, reticulated with veins, shining on both sides: germ. stalked, ovate-lanceolate, smooth.

$$
\text { ** Adult leates entire, nearly smooth. }
$$

L. orbicular, sonewliat elliptical, obtuse, coriaceous, with reticulated veins; glaucous beneath: germ. sessile, downy.

$$
\text { *****ar Leates all shaggy, woolly, or silly. }
$$

i L.nearly entire, elliptic-lanccolate; woolly and snow-white beneath : petioles decurrent: germ. sessile, ovate, woolly.

B. nearly entire, ovate-lanceolate, acute; shaggy above, densely silky, somewhat cottony, bencath: style as long as the almost sesile, woolly germen: stig. capillary, decply divided, as long as the style.

9 L. nearly entire, ovate, acutc; reticulater abovc; vciny and densely woolly bencath: stylc as long as the sessile, woolly germen: stig. linear, divided, the lengtli of the style.

L. roundi-li-ovate, pointed, entire; slagery on both sides; glaucous beneath: genn. sessile, oblong, smootli: style 4 times as long as the blunt, divided stig.

1 Fiect: I. elliptical, entirc, somewhat revolute, with a recurverl point ; silky and shining bencath, as well as the branches: gernn. ovatelanceolate, its stalk silky: stylc not longer than the stigmas.

2 Recumbent: L. elliptical, nearly entire, with a recurved point; glaucous and silky beneath: germ. ovate-lanccolatc, on a silky stalk.

Stem depressed, with short upright branches: L. elliptic-lanccolate, straight, somewhat pointed, nearly entire; glauccus and silky beneath: stip. 0: germ. stalkcl, ovatc, downy : capss. smooth. 
CLASS XXII. DIOECIA.

ORDER II. IYIANDRIA.

\begin{tabular}{|c|c|c|c|c|c|c|}
\hline LINNEAN NAMES. & ENGLISH NANIES. & 它鹤 & 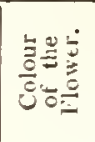 & 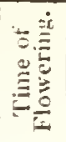 & 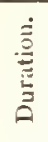 & 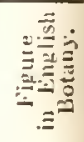 \\
\hline 44 fusea $\ldots .$. & brownish dwarf . & Nor.hea. & & 5 & S. & 1960 \\
\hline 45 prostrata ... & early prostrate... & Heaths & & 3,4 & S. & 1959 \\
\hline 46 inculsacea.... & trailing silky & San. mea. & & 5 & S. & \\
\hline 47 Doniana .... & rusty-branched .. & Scotland & & 5 & S. & \\
\hline 48 rosmarinifolia & rosemary-leaved & Moi. san. & & 4 & S. & 1365 \\
\hline 49 cinerea ..... & grey sallow ...... & Moi. wds. & & 4 & T. & 1897 \\
\hline 50 aurita ..... & trailing sallow & Moi. wds. & & 4,5 & S. & 1487 \\
\hline 51 aquatica ... & water sallow $\ldots$. & Swamps & & 4 & 'T. & 1437 \\
\hline 52 oleifolia $\ldots$. & olive-leaved $\ldots$. & lledges & & 3 & $\mathrm{~T}$. & 1402 \\
\hline 53 cotinifolia... & quince-leaved.... & Wocds & & 4 & s. & 1403 \\
\hline 54 hirta....... & lnairy-branched & Hedges & & 4,5 & $\mathrm{~T}$. & 1404 \\
\hline 55 rupestris ... & silky rock.. & Sc. rocks & & 5 & S. & 2342 \\
\hline 56 Andersoniana. & green mountain & Nor. wds. & & 4,5 & $s$. & 2343 \\
\hline
\end{tabular}


SIFCHFICHARACTER.

\section{SALIX.}

4 Frect, mueh branched: L. elliptie-oblong, aeute, flat, with a few glandular teeth; glaueous and silky bencath: stip, 0 : germ. sessile, nearly smooth, tapering into an elongated style.

Prostrate, with long straight branches: L. elliptie-oblong, convex, toothed, with a eurved point, glaucous, silky, and veiny beneath : stip. 0: germ. stalked, ovate, silky: style shorter than the stig.

Recumbent: L. elliptie-lanceolate, pointed, nearly entire; eonvex above, with prominent reticulated veins; glaueous and silky beneath : eatkins ovate-oblong: germ. silky, on long stalks: caps. smooth.

7 Ereet: L. obovate-laneeolate, partly opposite, acute: livid and silky beneatlı: eatkins eylind. germ. stalked, silky, longer than the bearderl scales.

Erect: L. linear-lanceolate, aeute, straight, entire; silky beneath : eatkins ovate, reeurved: germ. stalked, laneeolate, silky.

9 Frect: L. lower entire; upper serrated, obovate-lanceolate; glaucous downy, and reticulater with veins beneath: stip. half-shaped, serrated: germ. silky; its stalk half as long as the laneeolate seales.

0 Branches trailing: L. obovate, obtuse, with a small hooked point; eonvex, somewhat serrated; hairy and reticulated on both sides: stip. roundish, convex, toothed: germ. silky, stalked: stig. nearly sessile.

1 Erect: L. obovate, slightly serrated, flat, minutely downy, rather glaucous beneatlr: stip. rounded, toothed: germ. silky, stalked: stig. nearly sessile.

2 Erect; branclies spreading: L. obovate-laneeolate, flat, acute, rather rigid, minutely toothed; glaueous, retienlated, and hairy beneath: stip. small, notched, rounded: eatkins oval.

13 Ereet; branches spreading, downy: L. roundish, slightly toothed; glaueous and downy, with reetangular veins, beneath: style as long as the linear, notched stigmas.

Ereet; branches hairy: L. elliptie-cordate, pointed, crenatc; downy on both sides: stip. semi-eordate, flat, toothed, nearly smooth.

15 Trailing; branches downy: L. obovate, aeute, flat, serrated ; silky on both sides : stip. hairy: germ. stalked, awl-shaped, silky: style as long as the blunt undivided stigmas.

"6 Upright; branehes downy: L. elliptical, aeute, finely notched, rather downy; paler beneath: strp. half ovate, nearly smooth: germ. smooth, stalked: style cloven, longer than tlie cloven stigmas. 


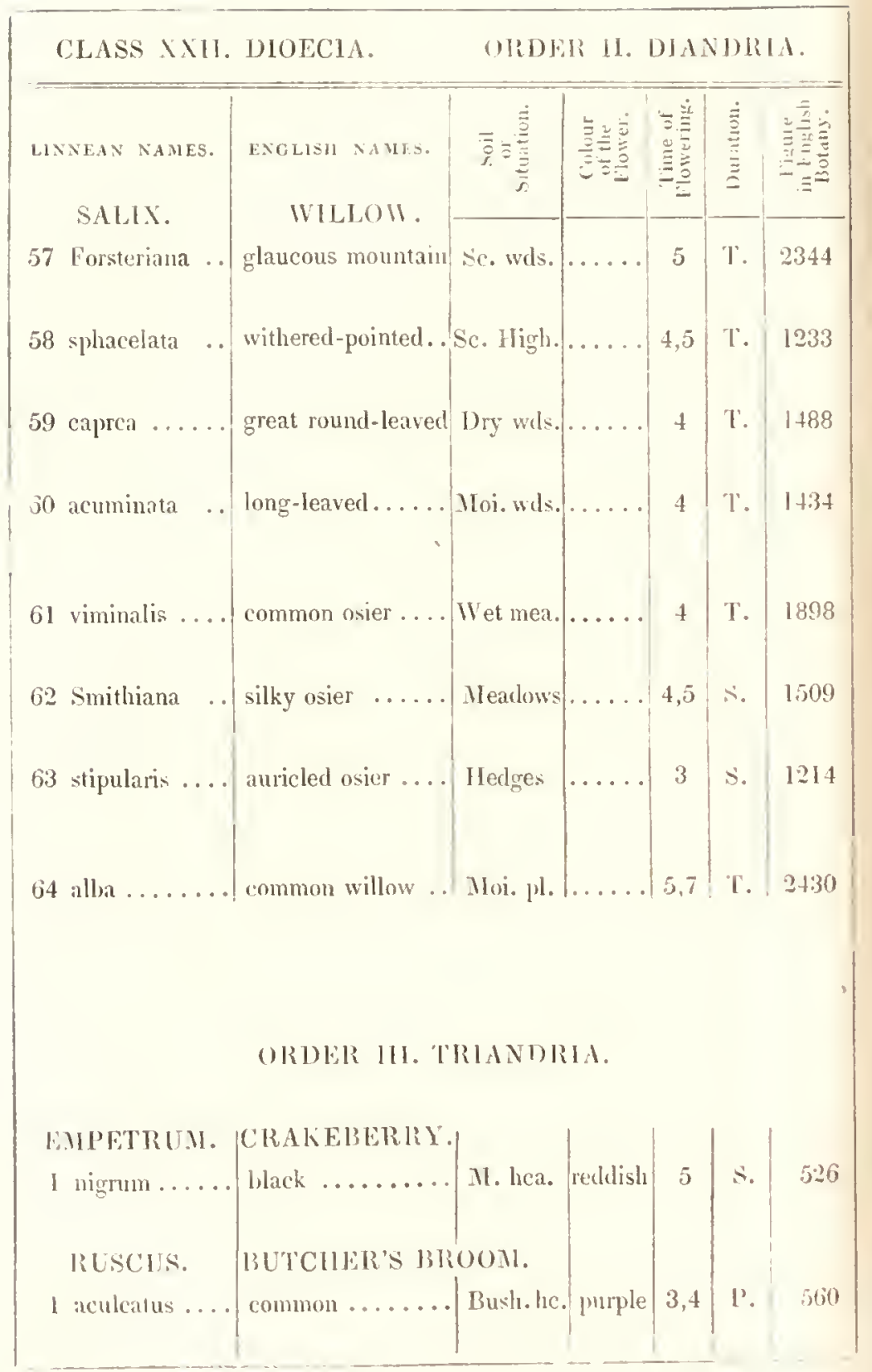


SALIX.

7 Frect; branches downy: L. olsovate, acute, crenate, downy; glaneous beneath: stip. vaulted: germ. stalked, awl-shaped, silky: style as long as the blunt, notehed stigmas.

8 Erect: L. elliptic-obovate, veiny; downy on both sides; discoloured at the point: stip. semi-cordate, toothed, erect: germ. stalked, orate-laneeolate, silky: stig. notched, longer than thc style.

9 Erect: L. roundish-orate, pointed, serrated, waved: pale and downy bencath: stip. Iunate, eatkins oval: germ. stalkcd, ovate, silky: stig. ncarly scssilc, undivided: caps. swelling.

0 Ereet: L. lanceolatc-oblong, pointed, wavy, finely-toothed; glancous and downy beneath: stip. half ovate, then reniform: eatkins cylincl: germ. stalked, ovate, hairy: stylc as long as the undivided stigmas.

l Branches straight and slcnder: L. linear-laneeolatc, taper-pointed, entire, wavy; white and silky beneath: germ sessile: style as long as the linear undivided stigmas.

2 L. laneenlate, pointed, wavy, tonthed; soft above; whitish and silky leneath: stip. lunate, minutc: eatkins ovate: germ stalked: style shorter than the linear, eloven stigmas.

3 L. laneeolate, pointed, wavy, obseurely crenate; soft abovc; white and downy beneath: stip. large, stalked, semi-cordatc: neet. cylind: germ. ovate, neasly sessife, as well as the linear undivided stiomas.

4 L. elliptie-lanceolate, pointed, scrrated; silky on both sides: the lower serratures glandular: stam. hairy: germ, smooth, nearly sessile: stig. deeply cloven: scales rounded.

\section{ORDER III. TRIANDRIA.}

\section{EMPETRUM. Empetrea. Nitt.}

II Stem and branches proeumbent: L. slightly elliptical.

451. RUSCUS. Smilacer. R. 13R.

I L. ovate, with a sharp horny point, flowering on the upper side, with out a leaflet. 
CLASS XXII. DIOECIA.

ORDER IV. TETRANISHA.

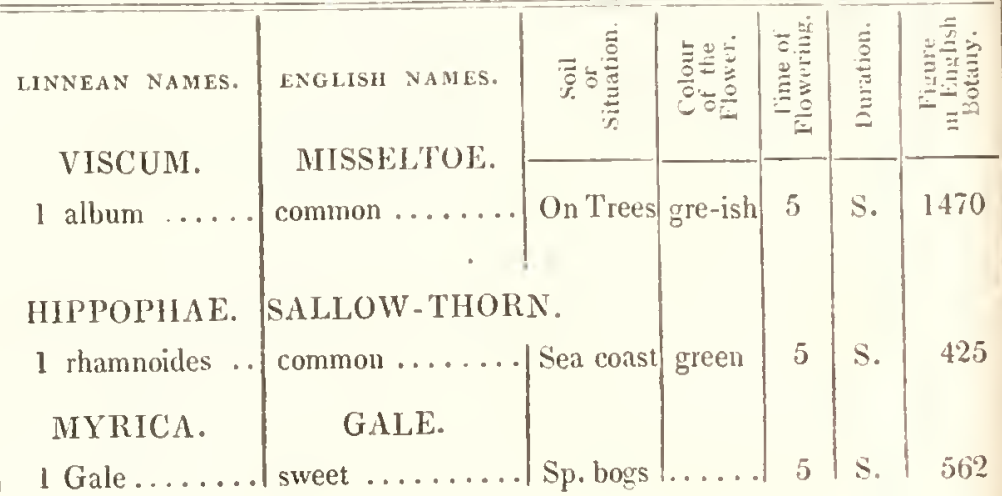

ORDER V. PENTANDRIA.

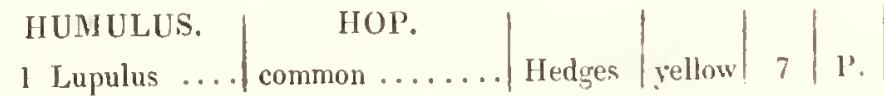

ORDER VI. HEXANDRLA.

ORDER VII. OCTANDRIA.

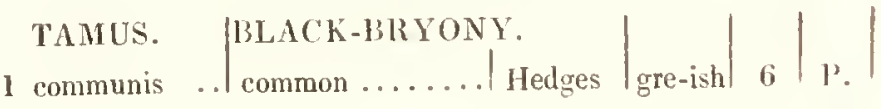

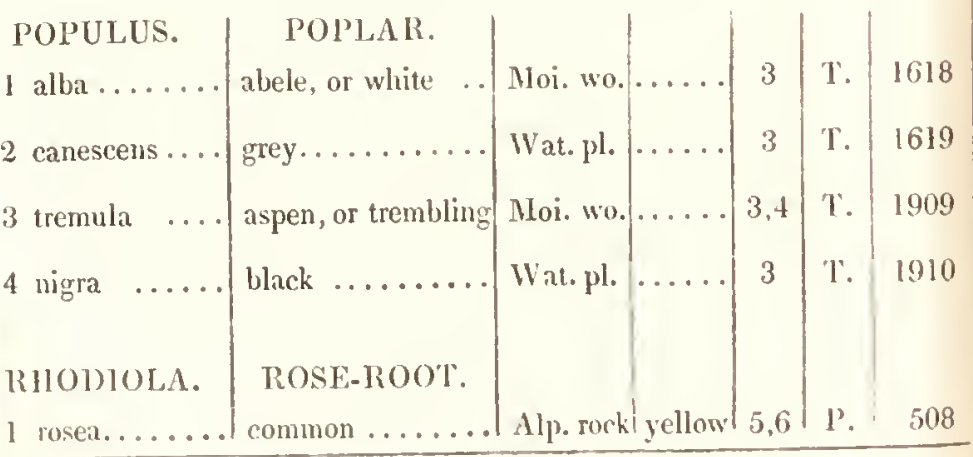




\section{SPEEIFIC CIIARATER.}

452. V1SCUM. Loranthea. Juss.

1 L. obovate-lanceolate, obtuse: stem diehotomous, with sessile intermediate heads of about 5 Howers.

453. IIIPOP'HAE. Elaragnea. Juss.

I L. linear-lanceolate, scattered.

454. MIYRICA. Amentacer. Juss.

1 L. laneeolate, slightly serrated; tapering and entire at the base: seales of the eatkins pointed.

\section{ORDER V. PENTANDRIA.}

455. HUMULUS. Urticer. Juss.

1 L. coldate, serrated ; undividerl or 3-lobed.

ORDER VI. IIEXANDRIA.

456. TAMUS. Smilaced". R. 13R.

1 L. heart-shaped, undivided, acute.

\section{ORDER VII. OCTANDRIA.}

457. POPULUS. Amentacer. Juss.

1 L. sornewhat heart-shaped, lobed, toothed, tomentous and snow-white beneath: fertile eatkins ovate: stig. 4 .

2 L. roundish, decply waved, toothed, tomentous and hoary beneath: fertile catkins cylind: stig. 8.

3 L. nearly orbicular, toothed, smooth on both sides: petioles compresserl: young branches hairy: stig. 4 . ereet, auricled at the base.

4 L. deltoid, pointed, serrated, smooth on both sides: catkins eylind. lax: stig. 4 , simple, spreading.

458. RHODIOLA. ('russularea. DE C.

1 L. obovate pointer, Acshy, glaucous. 


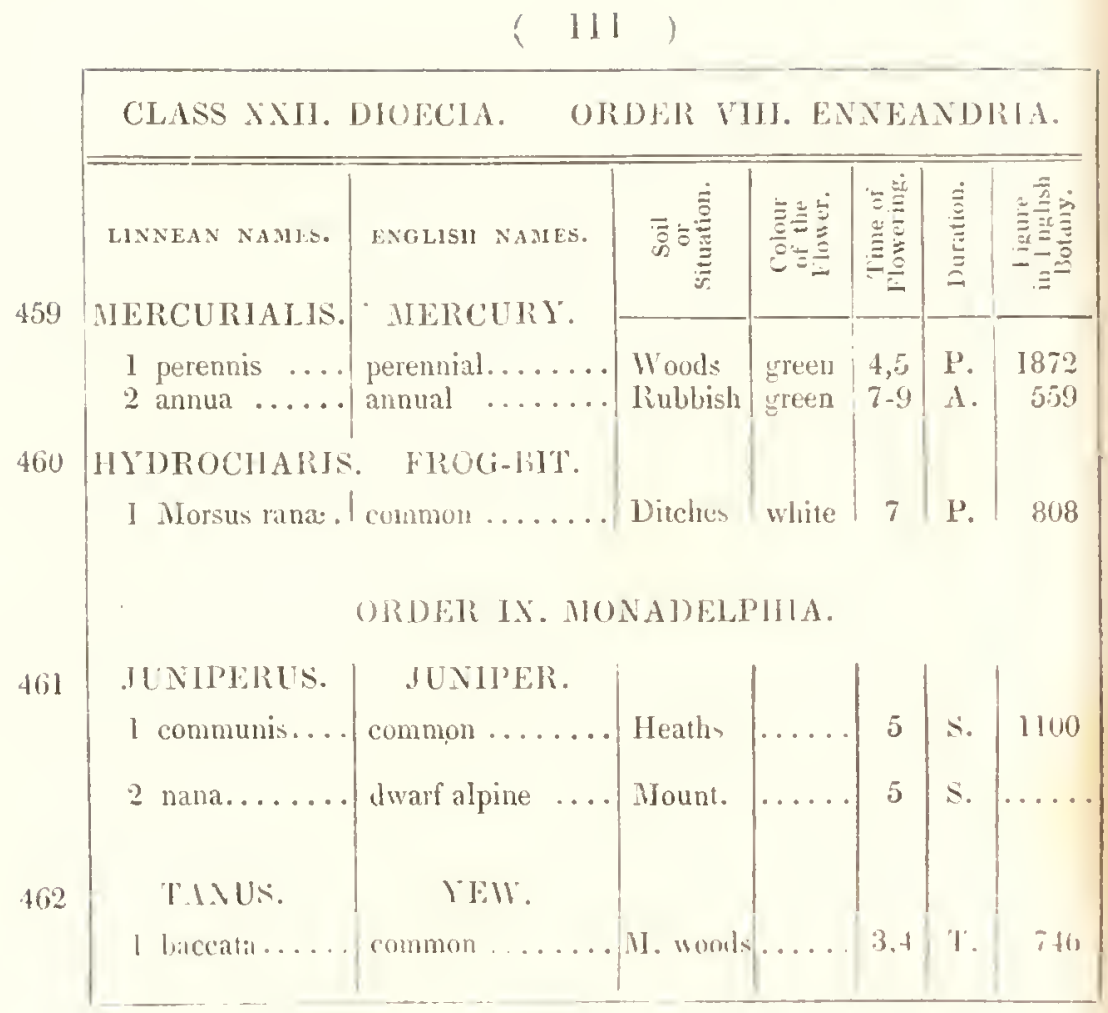


ClASS XV11. DOECIA. ORDER VHI. ENNGANIIRIA.

SPECIFIC CHARACTER.

459. MFIRCURIALIS. Eaphorbucer. IUSs.

1 stem quite simple: $1_{\text {. }}$ rough : root creeping.

2 Stem cross-branching: L. smooth: bar. fl, spiked: root fibrous.

460. HITROCllARIS. Hydrocharider. IUss.

1 L. entire, kidney-shaped.

ORDER I...NADELPHIA.

461. JUNIPERUS. Comiferk. Juss.

1 L. 3-together, spreading, tipped with a spine, longer than the berry: stem erect.

2 L. 3-together, tipped with a spine; somewhat imbricated, curved; the length of the oval, ripe fruit: stem recumbent.

462. TAXu's. Comiferer. Juss.

I L. approsimate. 2-ranked, linear, flat: recept. of the barten flowers globular. 



\title{
CLASS XXIII.
}

\author{
POLYGAMIA.
}

\section{ORDER I. MONOECIA.}

033 Aтriplex. Androgynous fl. Cal. 5-parted, inferior. Cor. 0. Stam. 5. Style cloven. Seed 1, depressed.

Fertile fl. Cal. 2-leaved, inferior. Cor. 0. Style cloven. Seed 1, compressed. 
CLASS XXIII. POLYGAMIA. ORIDER I. MONOLCIA.

\begin{tabular}{|c|c|c|c|c|c|c|}
\hline LINNEAN NAMES. & ENGLISH NAMES. & 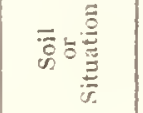 & 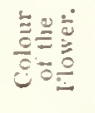 & 政 & 离 & 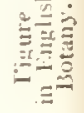 \\
\hline $\begin{array}{l}1 \text { portulacoides. } \\
2 \text { laciniata .... }\end{array}$ & $\begin{array}{l}\text { shrubby } \ldots \ldots \ldots \\
\text { frosted sea ...... }\end{array}$ & $\begin{array}{l}\text { Mud. sl. } \\
\text { San. sh. }\end{array}$ & & 7,8 & $\therefore$. & $\begin{array}{l}261 \\
165\end{array}$ \\
\hline 3 patula & spreading & Dunghill & & $6-8$ & 1. & 936 \\
\hline 4 angustifolia & narrow-leaved.... & Rubbish & & 6,8 & A. & 1774 \\
\hline 5 erecta... & upright $\ldots \ldots \ldots$ & Fields! & & 8 & A. & 222 \\
\hline 6 littoralis & grass-leaved & Mud. sh. & & 8,9 & A. & 70 \\
\hline 7 pedunculata. & stalked sea. & Salt mar.! & & 8.9 & F. & 232 \\
\hline
\end{tabular}




\section{SPLCIFIC CHARACTER.}

463. ATRIPLEX. Chenopodec. DE C.

I Sten shrubby: L.opposite, obovate-lanceolate: fil.mostly monoecious.

2 Stem herbaceous, spreading: L. deltoid, angutar and tuothed, scaly beneatl.

:3 Stem herbaceous, spreading: L. deltoid-lanceolate, somewhat hallsertshaped: ealyx of the seeds more or less muricated on the tisk.

4 Stem herbaceous, spreading: I. laneeolate, entire: lowcrmost somewhat hastate: calyx of the sceds hastate, slightly warty at the sides.

5 stem herbaceous, ereet: L. ovate-laneeolate; lower ones sinuated: ealyx of the seed inuricated on every side.

6 Stem herbaceous, erect: L. all linear, entire, toothed, or sinuated: ealyx of the seed muricated, sinuated.

7 Stem herbaceous, flexuose, divarieating: L. obovate, entire: fertilc f. peduncled, wedge-shaped. 


\title{
CLASS XXIV.
}

\author{
CRYPTOGAMIA.
}

\section{ORDER I. FILICES. Ferns.}

Frond with either dorsal, terminal, axillary, or radical jructificution.

- Capsules aggregate, on the back of a leafy fromd, bivalve, each bound with an elastic ring, contrary to the valies.

464 Polypoulum. Caps. in ronndish, scattered masses. Corer none. 465 Asprorum. Caps. in roundish, scattered masses. Cocer nearly orbicular, fixed by the centre, separating all round.

466 Cystea. Caps. in roundish, scattered masses. Cover orbicular, concave, fixed by a latcral point underneath; finally reflexed and jagged.

472 Woodsia. Caps. in roundish, scattered masses. Cover roundish, fixed by a central stalk under each mass. The margin in numerous, deep, capillary, incurved segments. 467 Aspleniun. Caps. in linear, scattercd masses. Cover lincar, separating at the side towards a nidrib or vein.

468 Scolopendrius. Caps. in nearly linear, twin, scattered musses, between 2 parallel veins. Cocers 2 , linear, opposite, folding one over the other.

469 Brecnnum. Caps. in linear, solitary musses, close to the midrib. Cover linear, flat, bursting towards the rib.

470 Preris. Caps, in linear masses, near tle margin. Cover from the inflexed margin of the frond; wavy, continuous, bursting inwards.

471 Anlantum. C'aps. on thic back of each rounded, reflexed, marginal, distinct coter, in small, roundish, central musses.

473 Tricionanfs. Caps. in oblong masses, imbedded in the margin of the frond. Cover pitcher-shaped, 1-leafed, opening outwards.

474 Hymkophyelun. ("aps. In roundish masses, imbedded in the margin of the fromd. Cover marginal, 2-valved, compressed, opening outwards.

** C'apsules, or seeds, withut uny distinct clastic ring.

479 Equsetun. Catkins tcrminal. Sects scparately embraced by 4 spiral filements, presunicd to hear pollen.

477 (1)nugrossum, Spike 2-ranked. Caps. 2-valved, imbedded.

475 Osaunda. Cluster hranched. Caps. globular, stalked, 2-ralied. naked, with a striated protulurance.

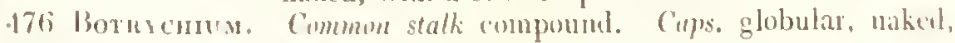
cimple, sesile, ?-vilvert. 
78 Lусоролиз. Cups, axillary, solitary, compressed, 2-valved. 80 P'ntrunia. Common receptacle 4-celled, concealing many barren and fertile florets. Sceds eoated.

481 Isoers. Common receptacle 1-eelled, in the base of the frond. seeds rough. 
CLASS XXIV. CRYPTOGAMIA.

ORDER I. FILICES.

\begin{tabular}{|c|c|}
\hline LINNEAN NAMUS. & ENGLLH XAMUS. \\
\hline OLYPOIIUA. & POLYPOI)Y. \\
\hline
\end{tabular}

$\mid$

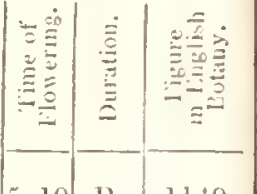

1 vulgarc........

common

Sha. banks, Sic. 5-10 P.

$11+9$

2 Phcgopteris....

pale mountain.... Mountains . . 6,7 , ${ }^{\prime \prime}$.

2224

3 Dryopteris ....

three-branchert

4 calcareum

rigid

Shady hills..

7 P.

616

ASPIJIUN.

1 Lonchitis

SHELD-FERN.

r roug

rough alpine ....

2 Thelypteris

.

$\operatorname{marsh} . . . . . .$.

Calca. rocks

7

P.

152,5

3 Oreopteris

h h

. Hea

Alp. rocks .

5,6

P. * * 97

I

Marshes! .

7

P. ${ }^{*} 1018$

4 Filix mas.....

malc

.

Heaths

7

P. *1019

5 cristatum

less

lesscr-crested ....

Shady places

6,7

P.

1458

Bog

6 aculeatum

common prickly..

Sharly platecs

6 P

1.562

7 angulare

a angular-leaver

.

Shady plices

8 lobatum

closc-leaved

9 spimulosim ....

crested

s

N

Marshes!

10 lilatatum .......

grcat-crested

Wet shady pl. 6-8

P.

2125 (1) 11 dumetorum .... thicket......... Bushy places 


\section{POLYPODIUM.}

1 Frond deeply pinnatifid: lobes oblong, somewhat serrated, obtuse : root scaly.

2 Frond pinnatc: leafl. lanceolatc, pointed, pinnatificl, united at their base: the lower pair reftexed: ribs and veins hairy. Sori marginate.

F Frond 3-branched, bipinnate, sprearling-reficxed ; lobes obtuse, somewhat crenate: root filiform: scattered, distinct.

It Frond 3-branched, bipinnate, ereet, somewhat rigid; lobes obtuse, slightly crenatc. sorri conflucnt.

\section{ASPIDITM.}

il Frond linear-lanceolatc, pinnatc; leafl. somewhat lunate, bristly scrrated, bent downwards: stalks sealy. Polypodizm. Eng. Bot.

2 Frond pinnate: lcafl. pinnatifid, somewhat crenate, smooth, with ovate lobes. Suri globular, finally confluent: cover central, lax: root trailing. P'olupodium. Eng. Bot.

3 Frond pinnate; lcaft. pinnatifid, entire, with resinous glands beneath. Sori nearly marginal, finally eonfluent: root tufted. ''olypodium. Eng. Bit.

4 Frond hipinnate; leafl. obtuse serrated: stalk scaly. Smi near the nidrib: cover orbicular.

5) Frond finnate; leafl. pinuatifid, acute; lobes ovate, blunt, crenate, sharply toothed: stalk sealy at the basc. Sori lialf way between the midrib and margin: cover orbieular.

6 Frond bipinnate; leafl. ovate, somewhat lunate, fringed with spines; hairy beneath: common stalk scaly: cover orbicular, flat.

7 Frond bipinnatc: lenfl. ovatc, obtusc, stalked, fringed with bristly serratures; and having a lateral lobe at the base: lowermost partly pinuatifid: stalk scaly : ribs shaggy : cover orbieular, umbilicated.

8 Frond bipinnate; leatl. elliptieal, decurrent, slightly lunate, serrated; lower pair very large: stalk sealy: cover orbicular, umbilicated.

!9 Frond triangular, bipinnate; leafl. elliptical, dccurrent, pinnatifid, tipped with small spines: stalks scaly at the base: ribs zig-zag: cover orlicular, witl a lateral notch.

0 Frond bipinnate; leafl. pinnatifid, decply serrated, tippel with small spines: stalk soaly: eover reniform, tumid: finally orbicular, with a latcral notcl.

1 Frond bipinnate; leaf. pinnatifid; lobes with sharp, terminal, prickly teeth: stalk scaly: cover orbicular, fat, with a deep noteh. 
CLASS XXIV. CRYPTUGAMIA.

ORDER I. FIJICES.

1

I.INNEAN NAMES.

ASPIDIUM.

12 Felix formina female........

13 irrigum $\ldots . .$.

ENGLISH NAMES.

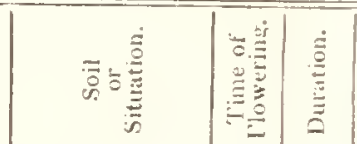

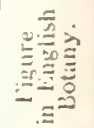

\section{ASPLENIUM.}

SPLEEN WORT.

1 'Trichomanes .. com. maiden hail'.

Wet shady pl

6,7

1459

brook I

BI ADDER-FERN.

1 fragilis.......

brittle

Shady rocks

6,7

P.

*1587

2 dentara.

toothed

Alpinc rocks

3 augusta1a.....

mountain

. Sha

4 regia.........

laciniated

Wallsirocks!

1.7 $\mathrm{I}^{\mathrm{T}}$

2199

2 viridle

3 malinum

g

green maiden hair .

Sharly rocks

$3,11 \mathrm{P}$.

576

Mlp. rocks .. 6,7 P.

2257

4 septentrionalc .. sca ...........

5 alternifolium ..

forked .........

6 Ruta-inuraria ..

alternate-leaved ..

Rocks..... 6-10

Scutcl rocks! 6-10

P.

1017

2258

7 Adiantum nigrum black maidcn hair.

.

shady rocks

I'.

150

Slady places

P.

1950

8 lanceolatun.... lanceolate ......

Rocks s walls 6-10 P

240

9 fontanum...... smooth rock .... Shady rocks!

$6-9 \quad$ P. $* 2024$

NCOLOPEN DRIUAL. IIAR'T'S-TONGUE.

1 vulgare.......

2 Cetcrach ..... scaly ........ Cillc. rocks.. $3-12$ | 
ASPIDIUM.

.2 Frond bipinnate; leafl. pinnatifid, serrated, pointless: stalk smooth; eover oblong, finally reniform, jagged.

3 Frond laneeolate, pinnatc; leafl. deeply pinnatifid, cut, tootlicd: stalk quadrangular: cover oblong; finally reniform, jagged.

\section{CYSTEA.}

I Frond oblong-lanceolatc, bipinuate; leafl. ovate, aeute, pinnatifid, cut, or scrrated : midribs bordered. Sori erowded: cover irregularly torn. Cyatheu. Eng. Bot.

2 Frond oblong-laneeolate, bipinnate; leaf. ovate, obtuse, bluntly toother, partly pinnatifid: partial midribs bordered. Sori crowded, finally eonfluent. Cyathea. Eng. Bot.

3 Frond oblong, bipinnate: leafl. lanceolate, decurrent, wavy, or pinnatifid, with linear, acute, partly cloven scgments. Sorri seattered; permanently distinet.

4 Frond lanccolate, bipinnate; leafl. deeply pinnatifid, with oblong, blunt, partly notehed segments: partial stalks winged. Sori many, scattered, permanently distinet. Cyathea. Eng. Bot.

\section{ASPLENIUN.}

1 Frond pinnate; leafl. roundish, crenate: midrib coloured, polished, keelerl.

2 Frond pinnate; leafl. roundish, erenate: midrib flattcned beneatl.

3 Frond pinnate; leafl. ovate, oblique, serrated, blunt, unequal and wedgc-shaped at the basc.

4 Frond wing-3-cleft; segments alternate, linear, jagged at the tops.

5 Frond pinnate; lethlolternate, wedge-shaped, erect, notched at the tops.

6 Frond altcrnately twice eompound; leat. rhomboid-wedge-shaped, spreading, notched at the tops.

7 Frond somewliat deltoid, alternately thrice pinnatc; leafl. laneeolate, sharpish, decply serrated.

8 Frond lanecolate, alternately twiee pinıate; lcafl. obovatc, deeply and sharply serrate: principal midrib not bordered.

9 Frond linear-lanccolate, bipinnate: leafl. wedge-shaped, sharply toothed: midribs all bordered. Adiantum. Eng. But.

\section{SCOLOPENDRIUM.}

I Frond oblong, smooth, curdate at the base: stalk shaggy.

2 Frond pinnatifid, lanceolate, sealy beneatl. 
CLASS XXIV. CRYP'TUGAIIIA.

ORJUAR I. FILICRS.

\begin{tabular}{l|l} 
LiNNEAN Names. & ENGLish Names. \\
BLECHNUM. & BLECHNUM.
\end{tabular}

1 boreale ........

northern .........

TRICHOMANES. HAIR-FERN.

l brevisetum. .... short styled......

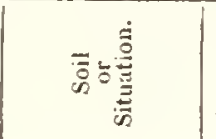

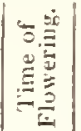

lleaths ....

7 P. 1159

1 aquilina ......

BRAKE.

2

PTERIS.

common ...... Hea, and wo.

crisp

ADIANTUM.

1 Capillus Veneris

rock $\ldots . . . \cdots$
MAIDEN-HAIR.

Stony hills ..

WOODSIA.

1 ilvensis........

2 hyperborea...

oblong ........ Alpine rocks

$7-9$

Rocks! ....

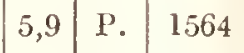

IIYMENOPHYLLUH. FILMY-FERN.

1 Tunbridgense .. $\mid$ Tunbridge ...... Moist rocks..

Alpine rocks!

Moist rocks!

5,6

P. ${ }^{*} 1417$

**

OSMUNDA.

OSMUND ROYAL.

I regalis ....... common ....... Boggy places

\section{BU'TRYCHIUN. MOONWORT.}

1 Lunaria ...... common ........ Mtount. pas.. 


\section{SPECIFIC CHARACTER.}

\section{BLECHNUM.}

I Frond pinnate, pectinate, smooth; leaf. linear, bluntish, entire, scarccly dilated at the base.

\section{PTERIS.}

I Frond thrice pinnatc: leaf. lanccolate, bluntish; the lowcrmost pinnatifid ; the uppermost smaller ; term. ones large, undivided.

2 Frond twice or thrice pinnate; barren leafl. wedge-shaped, cut; fertile ones elliptical, blunt, convex.

\section{ADIANTUM.}

I Frond alternately twice-compound; leaft. stalked, wedge-shaped, lobed: covers transversely oblong.

\section{WOODSIA.}

Brown, Trans. Limn. Soc, vol. xi.

Leaf. oblong, pinnatifid, with oblong segments.

D Leafl. Jluntly triangular, pinnatifid, with rounded segments. Polypodium. Eng. Bot.

\section{TRICHOMANES.}

I Frond thrice pinnatifid, lobed, smooth; segm. lincar, entire: stalk winged: covers oblong. Hymenophyllum. Eng. Bot.

\section{IIYMENOPHYLLUM.}

1 Frond lipinnatifi, smooth; segmentsand covers serrated : fruct. solitary, from the upper edge of the lowernost of the secondary segments.

* Capsules, or seeds, without any distinct elustic ring. Mabit various.

4\%. OSMUNDA.

1 Leaf. oblong, nearly entire; lilated, and somewhat auricled at the base: clusters panicled, terminal.

\section{BOTRYCHIUM.}

"1 Leaf solitary, pinnate : leaf. fan-shaped, notched. Osmunda. Eng. Bot.

\section{OPIIIOGLOSSUM.}

11 Leaf ovate, veinless, about as tall as the spikc. 
CLASS XXIV. CRYPTOGAMIA.

ORIER 1. FILICLS.

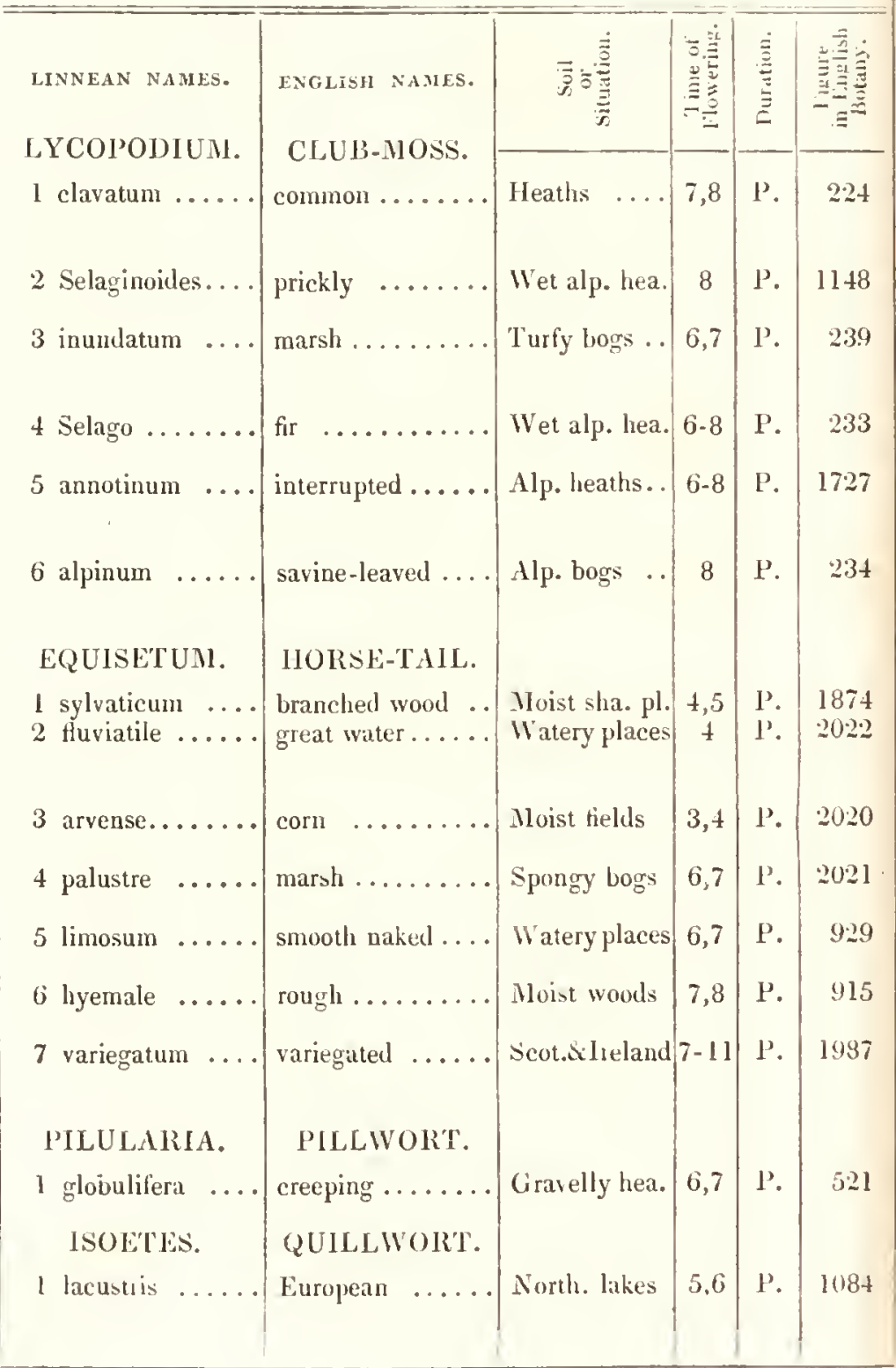




\section{LYCOPODIUM.}

1 Stem creeping: branches ascending: L. scattered, incurved, with thready points: spikes $2-3$ cylind. stalked; with dilated membranous scales.

2 Stems recumbent, branched; shorter than the solitary, ascending, tumid spikes : L. scattered, lanceolate, fringed; floral ones larger. 3 Stems depressed, creeping, slightly branched: L. scattcred, linearlanceolate, pointless, entire: spikes solitary: scales dilated at the base.

4 Stems erect, forked, level-topped: L. in 8 rows, uniform, lanceolate, pointless, entire, slightly spreading.

5 Stems recumbent at the base : branches erect; anuually proliferous at the summit: L. scattered, in 5 rows, lanceolate, acute, slightly serrated ; Horal ones broader than long, imbricated.

6 Stems prostrate: branches erect, clustered, forked, level-topped : L. in 4 rows, acute, keeled: scales ovate-lanceolate, flat.

\section{EQUISETUM.}

1 Branches compound, curved downwards, smooth.

2 Barren stems branched on all sides; branches roughish, angular: flowering stems unbranched, with many crowded sheatlis, deeply toothed.

3 Barren stems with angular, roughish branches : flowering stems unbranched; their sheaths distant; deeply toothed.

4 Stem furrowed, branclied throughout, with a term. catkin: branches simple, erect, roughish, with simple angles.

5 Stem partly naked, striated, smooth as well as the branches: catkin term. elliptical.

6 Stem naked, very rough, somewhat branched at the base: sheaths whitish, black at the base and top: teeth deciduous : catkin term.

7 stem naked, very rough, branched at the base: sheaths black, with white membranous, lanceolate teeth : catkin term.

$$
\text { 480. PILULARIA. }
$$

1 Stem creeping: L. filiform.

\section{ISOETES.}

1 Fronds bluntly quadrangular, with 4 longitudinal cells. 



\section{CIAAS XXIV. CRYPTOGAMIA.}

\section{ORDER II. MIUSCI.}

The reproductive organs of the Misses are of two kinds: the one, a capsule, with a lid, and a central axis, called the columella, to which the seeds are attached; the other, a minute spherical body concealed in the axils. Plants leafy.

\section{Tribe I. EVAGINULATI.}

Caps. sessile; its recept. stalked; without perichotial leaves.

A. Peristome none.

:82 Sphagnum. Cups. sessile; mouth naked; lid deciduous.

\section{Tribe II. VAGINULATI OLOCARPI.}

Caps. stallied; with perichatial leaves; not valvular.

$$
\text { * Fruit-stalks terminal. }
$$

183 Рнascua. Caps. entire. Recept. sessile. Lid persistent. Calyptra shorter than the capsule, dimiliate.

184 Schistostega. Caps. dehiscent, naked at the mouth. Lid lacinjated, with deciduous segments.

185 Gymosтоnum. Caps. dehiscent, naked at the mouth. Calyptra dimidiate.

B. Peristome simple.

* Fruit-stalk terminal. Capsules dehisecnt.

386 Нумехозтомим. Peristome destitute of teeth, but liaving an inner horizontal membrane, perforated in the middle.

1487 Tetraphis. I'eristome of 4 erect tceth.

1488 Excalypta. Peristome of 16 teeth. Calyptra cylindrico-campanulate, entirely covering the capsule.

489 Grimmis. Peristome of 16 entire or perforated, rarely cleft teeth. Calyptra mitriform. 
490 Wfissia. Peristome of 16 entire equi-distant teeth. Caluptra dimidiate.

491 Dieranum. Peristome of I6 bifid equi-distant teeth. Calyptra dimidiate.

492 Tinenostonum. Peristome of 16 equal teeth divided to the base, or 32 in pairs. Caluptra mitriform.

493 Cinclidotus. Peristome of 32 filiform twisted teeth, anastomosing at their base. Calyptra mitriform.

494 Tortula. Peristome of 32 filiform twisted teeth, more or less united at the base. Calyptra dimidiate.

495 DIDYMonos. Peristome of 16 bifid equi-distant teeth. Caluptra dimidiate.

496 Splaennum. Peristome of 8 double teeth. Caps. with an evident apophysis. Columella exserted, eapitate. Caluptra mitriform.

497 Conostonum. Peristome of 16 teeth, united at the tips, but separated at the base.

* Frrit-stalk lateral. Capsules dehiscent.

498 Рт́rogoniun. Peristome of 16 entire equi-distant teeth. Calyptra dimidiate.

\section{Peristome dorble. \\ * Frnit-stalk termimal.}

499 Orтнотвгенм. Peristome mostly double; outer one of 16 teeth approaehing in pairs; inner one (if any) of 8-16 eiliary proeesses. Calyptra mitriform. Columella eapitate.

500 Zyonox. Peristome double or simple: teeth in pairs. Calyptrat hooded.

501 Dinnscium. Peristome, onter with 16 teeth: teeth obseure. Cips. subsessile.

502 livxвauma. Stem none. Caps. oblique, gibbous. Peristomeouter one of many filiforn proeesses; inner one of a eonieal, plieate membrane. Calyptra mitriform.

50:3 Funar. Peristome oblique, outer and inner ones eath of 16 teetl; opposite to eaeh other.

504 Bлатramia. Caps. subglobose. Peristome-outer one of 16 teeth; inner one a membrane eleft into 16 bifid segments. Caluptra dimidiate.

505 l'onla. l'eristome-teeth separate, aeute; membrane with 16 506 proeesses, which are entire at the end without cilia. 506 linva. l'eristome-outer of 16 teeth; inner one of a membrame cut into 16 equal segments, with filiform processes often placel between them. Colyptra dimidiate. 
77 Toustricuva. Peristome-outer one of 32 or 64 equi-distant incurved teeth: inner one of a dense horizontal membrane connected with the outer teeth. Calyptra dimidiate.

** Fruit-stalk lateral. Caps, lateral.

08 Anictanguus. Caps. naked at the moutl. Calyptra mitriform.

09 Fissidrss. Peristome simple; teeth bifid. Caliptru smooth.

10 Lruconox. Peristome simple, with bifid processes.

11 Fontiasuls. P'eristome double: outer one of 16 teeth; inner one of 16 eiliary proeesses formed by transverse bars into a reticulated cone. Caluptra mitriform.

i12 Anowopos. Peristome double: the first of 16 teeth; the second of 16 ciliary processes arising from the teeth. Calyptra dimidliate.

;13 Neckera. Peristome double: outer one of 16 teeth; inner one of 16 ciliary processes connected only at the base by a short membrane. Caluptra dimidiate.

i) 14 Daltonia. l'eristome double: membrane figured, with 16 ciliary and reflexed teetl.

j15 Hоокеria. Peristome double: outer one of 16 teeth; inner one of a membrane cut into 16 entire segments. Calyptra mitriform.

316 LESKEA. Peristome double: membrane with 16 entire processes: teetl erect or reflexed. Caluptra hooded.

517 HYPNus. Peristome double: outer one of 16 teeth; inner one of a membrane eut into 16 equal segments, often with filiform processes between them. Calyptra dimidiate.

\section{TurBe III. VAGINULATI SCIISTOCARPI.}

Caps. stalked, with perichatial leaves, valvular.

518 AnnREs. Capsule 4-valved: valves cohering at the apex, and adhering to the persistent lid. 
CLASS XXIV. CRYPTOGAMIA.

ORDER 1I. MUSC1.

\begin{tabular}{c|c} 
Linnean Names. & ENGLISII Names. \\
SPHAGNUM. & BOG-MIOSS. \\
I obtusifolium.... & broad-leaved ....
\end{tabular}

SCHISTOSTEGA. SCHISTOSTEGA

1 pennata ..... feathery ...... Banks.....

\begin{tabular}{|c|c|c|c|}
\hline 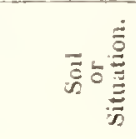 & 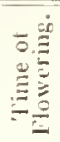 & 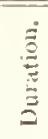 & 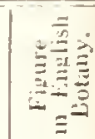 \\
\hline Bogs . & 6 & P. & $\approx 1405$ \\
\hline
\end{tabular}

PIIASCUAI. EARTIT-MIOSS.

1 subulatum .... awl-leaved ......

Dry banks ... 3-5

A.

2177

*2.219

GYMNOSTOMUM.BEARDLESS-MIOSS.

486 IIYMENOSTOMUM. IYMENOSTOMUM.

\begin{tabular}{l|l|l}
1 microstomum .. & small-mouthed .. & Banks... \\
TETHAPHIS. & FOUR-TOOTHED-MOSS.
\end{tabular}

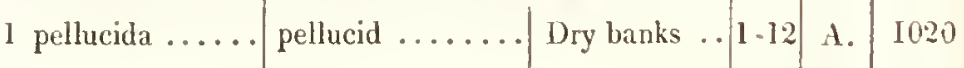

ENCALYPTA.

EXTINGUISHER-MOSS.

1 vulgaris common ........ Wall-tops ... 1-12

A. $* 558$

GIINIIIA.

GIIMIMIA.

1 pulvinata..... cushion ....... Housctops .. 1-12 
SIICIFIC CIIARACTER.

\section{THHe 1. LV'AgINULATJ.}

482. SPHAGNUM.

Branches tumid: L. ovate, obtuse. Sph. latifolizm. Ling. Bot.

For 3 other species, see Eng. Bot. 1406. 1498. 2392.

\section{TRIBE 11. VAGINULATI OLOCARPI.}

\section{PHASCUM.}

L. awl-shaped, straight; their nerve disappearing below the point. For 10 other species see Hooker's Musc. Brit. and Eng. Bot.

\section{SCHISTOSTEGA.}

Cymnostomum pennatum. Eng. Bot.

\section{GYMNOSTOMUH.}

L. ovate, pointed, flat, entire: lid obliquely rostrate.

For 13 other species, see Musc. Brit. and Eng. Bot.

\section{HYMENOSTOMUM.}

L. broadly subulate; margin involute; above flexuose, crisped when dry : caps. elliptical, contracted at the mouth; lid subulate, incurved. Gymnostomam microstomum. Eng. Bot.

\section{TELRAPHIS.}

1 Stens elongated: L.ovate-acuminate; those of the perichotium lanceolate: caps, cylindrical.

For another species, see Eng. Bot. 1422.

\section{ENCALYP'TA.}

I Stems short: L. oblong, obtuse; their nerve produced a little beyond the summits: caps. cylind. smootll: calyptra entire at the base. Bryum extinctorium. Eng. Bot.

For 3 other species, see Eng. Bot. Muse. Brit. and Grev. Cryp. Fl.

489. GRIMMHA.

I] Stems short, cushion-shaped: L. narrow elliptical; their margins recurved, hair-pointed: caps.ovate, striated, drooping : lid conical, acuminate.

For 6 other speries, sap MTuse. Brit 
CLASS XXIV. CRYPTOGAMIA.

ORDFR II. MUSCI.

TRICHOSTOMUN TRICIOSTOMUA.

I canescens......

FORK-MOSS.

I heteromallum ..

silky heath ...... Moist banks

$3-5$

A.

1272

Banks..... I -I2 I'. * I367

I controversa

disputed...

\begin{tabular}{|c|c|c|}
\hline 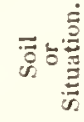 & 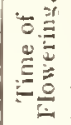 & \\
\hline & I - I 2 & I'. \\
\hline
\end{tabular}

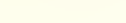

CINCLIDOTUS.

I fontinaloides....

CINCLIDOTUS.

water

S

Heath

l'.

2534

P'TEROGONIUN.

PTEROGONIUM

1 Smithii.

Smith's .......

TORTULA.

I muralis........ wall

Everywhere

I - 12

P.

2033

*557

5-8 P

557

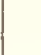

Trees...... I -12

P.

I326

SPLACIINUMI. GLAND-MIOSS.

. 1 ampullaceum .. bottle-headed .... 
490. WEISSIA.

1 Stcms nearly simple: L. linear, awl-shaped: caps. ovate-elliptical: lid rostrate. Grimmia, Eng. Bot.

For 18 other species, see Musc. Brit. and Grev. Cryp. Fl.

\section{DICRANUM.}

II Stens branched: L. capillary, leaning one way, 1-nerved, dilated at the basc: caps. ovate, somewhat drooping: lid with a long beak. For 22 other sprecies, see Musc. Brit, and Engr. But.

\section{TRICHOSTONUM.}

1 L. ovate-lanceolate, nerveless, channelled, with a pellucid point, slightly serrated: caps. ovate: teeth of the peristome long, filiform: lid subulate.

For 8 other species, see Musc. Brit. and Ling. Bot.

\section{CINCLIDOTUS.}

1 Fontinulis minor. Eng. Bot.

\section{TORTULA.}

1 Stem simple, short: L. linear-oblong, their margins recurved; hairpointed: caps. oblong: lid conical, acuminate.

For 10 other species, see Musc. Brit. and Eng. Bot.

\section{PTEROGONIUN.}

1 Stems much branched; branches pinnate, incurved by drying: L. entire: fruitstalks sliort: lid rostrate.

For 2 other species, see Eug. Bot. 1085, 2297.

\section{DIDYMIODON.}

1 Stems scarcely branched: L. lanceolate, carinate; their margins recurved, entire: caps. elliptical, obliçue, furrowed when dry, lid conical. Dicranum, Eng. Bot. For 9 other species, see Musc. Brit. and Grev. Cryp. Fl.

\section{SPLACHNUM.}

I L. ovate-lanccolatc, acute, scrrated: apophysis inversely flagonshaped, twice as wide as the capsule.

For 6 other species, see Hooker's Musc. Brit. and Eng. Bot. 
CLASS XXIV. CRYPTOGMMIA.

ORJJER II. MUSCI.

$=$

LINNEAN NAMES. ENGLISH NAMES.

CONOSTOMUM. CONE-MOUTHED-NOSS.

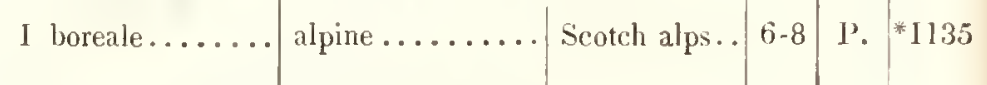

500

ORTHOTRICHUM BRISTLE MIOSS.

1 crispum $\ldots . .$. curled.........

Trees ......

\begin{tabular}{l|l|l} 
4-7 & I. & 996
\end{tabular}

$50 \mathrm{I}$

5112

\section{ZYGODON.}

I conoideum ....

\section{ZYGODON.}

DIPHYSCIUM.

club-fruited ....

DIPHYSCIUM.

I foliosum ......

leafy ..........

Woods ....

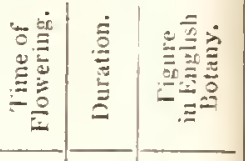

BUXBAUMIA.

BUXBAUMIA.

1 aphylla........

leafless

Fir wood.... 5-8

A. 1596

FUNARIA. CORD-MOSS.

I hygrometrica .. yellow twisting ..

Everywhere $10-4$

1. $* 342$

504

BARTRAMIA.

BARTRAIIA.

I pomiformis ....

apple .

Heaths

$4-8$

P.

*1239

Trees!...... 3,4

3-6

A. ${ }^{*} 329$

505

BRYUN.

1 androgynum....

\section{POHLIA.}

1 inclinata

inclined

BIRUM.

androgynons ....
Banks..... 3-5 P. *1238 


\section{LASS XXIV. CRIPTOGAMIA. ORDER II. MUSCI.}

SPECIFIC CHARACTER.

\section{CONOSTOMUM.}

L. lanceolate, acuminate, kceled, slightly toothed. Grimmia conostoma. Eng. Bot.

\section{ORTIIOTRICHUM.}

Stem branched: L. linear, much curled when dry: fruitstalks elongated: caps. striated: pcristome with 8 ciliary processes.

For 9 other species, see Musc. Brit. and Eng. Bot.

\section{ZYGODON.}

Mnium conoidenm, Eng. Bot.

\section{DIPII YSCIUM.}

Buxbaumiu foliosa. Eng. Bot.

\section{BUXBAUMIA.}

Caps. on a granulated stalk, rising from a hairy bulb.

\section{FUNARIA.}

L. concave, entire, ovate: nerve excurrent : fruitstalk curved, flexuose. Bryum, Eng. Bot.

For 2 other species, see Musc. Brit.

\section{BARTRAMIA.}

L. awl-shaped, serrated, with the nerve reaching to the tip, twisted when dry.

For 5 other species, see Musc. Brit. and Eng. Bot.

\section{POHLIA.}

L. ovate, acuminate, margins slightly rccurved: nerves running bcyond the points: caps. clongated, pyriform, pendulous. Bryum. Eng. Bot.-For 3 other species, see Musc. Brit.

\section{BRYUM.}

Stems nearly simple: I. lanceolatc, serrated, with the margins recurved: caps. nearly erect, sulcated: lid conical. Mnium. king. liot.

For 21 other species, sec Muse. Brit. and Eng. Bot. 
CLASS XXIV. CRYPTUGAMJA. ORDER 11. MUSCH.

POLYTRICIUM.

1 undulatum ....

HAIR-MIOSS. undulated ..... Shady banks

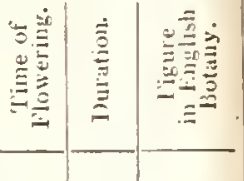

ANICTANGIUM.

ANICTANGIUN

1 ciliatum ciliate.

Ir. mountains $6-10$

P. $* 1179$

509

1 antipyretica .... nerveless.......

1 bryoides ..... bryum-like .....

Noist banks

LEUCODON.

WIITE-TOOTH.

1 sciuroides......

squirrel tail......

Trees ......

$5-8$

P. ${ }^{*} 1903$

\section{FONTINALIS. WATER-MOSS.}

Rivers.....

$6-8$

$1^{\prime}$.

359

\section{ANOMUDON.}

ANOMODON.

1 curtipendulum...

pendulous

.

Rocks $\alpha$ trees

4-7 P. *1444

\section{DALTONIA.}

DALTONIA.

1 heteromilla....

NECKERA.

1 pumila....... pigmy Trees! short-stalked

.

T'rees ......

$3-8$ I. * 1180

Moist banks

$4-7$ T. 1902 
SPECIFIC CHARACTER.

\section{POLYTRICHUM.}

1 L. lanceolate, undulatcd, serrated ; their nerve winged : caps. cylind. curved: lid awl-shaped.

For 9 other species, see Musc. Brit. and Eng. Bot.

\section{ANICTANGIUM.}

$1 \mathrm{~L}$. ovate, elongated, pellucid at the points; those of the pcrichotium laciniated at the extremity. - For another species, see Ling. Bot.

\section{FISSIDENS.}

1 Fruit-stalks terminal : perichœtial leaves resembling the cauline ones.

\section{LEUCODON.}

1 L. closely imbricated, ovate-cordate, acuminate, striated: caps. oblong. Dicranum, Eng. Bot.

\section{FONTINALIS.}

1 L. nerveless, for the most part complicate-carinatc.

For 2 other species, see Eng. Bot. 1861, 2432.

\section{ANOMODON.}

1 L. ovate, pointcd, serrulated, even, spreading: fruitstalks as long as the perichoetium: caps. ovate. Neckera, Kng. Bot. For another species, see Eng. Bot. 265.

\section{NECKERA.}

I L. in 2 rows, ovate, slightly undulatcd, margin recurved: fruitstalks scarcely longer than the perichotial leaves.

For 2 other species, see Eng. Bot. 617, and Crev. Cryp. Fl.

\section{DALTONIA.}

1 L. ovate, acute: caps. sessile, immcrsed: calypt. nearly entire. Neckera, Eng. Bot.

For another species, see Eng. Bot. 2564.

\section{HOOKERIA.}

1 L. ovate, entire, obtuse, nerveless.-For another species, see Musc. Brit. 


\section{CLASS XXIV. CRYPTOGAMIA.}

ORDER II. MUSCI.

\begin{tabular}{|c|c|c|c|c|c|}
\hline LINNEAN NAMES. & ENGLISH NAMES. & 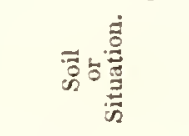 & 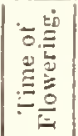 & $\stackrel{3}{3}$ & 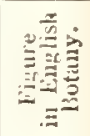 \\
\hline 1 complanata .... & flattened ........ & Trees ..... & 3,4 & P. & * 1492 \\
\hline $\begin{array}{l}\text { HYPNUM. } \\
1 \text { riparium ...... }\end{array}$ & $\begin{array}{l}\text { FEATHER-MIOSS } \\
\text { water .......... }\end{array}$ & Ditch banks & $5-11$ & P. & 2060 \\
\hline $\begin{array}{l}\text { ANDREEA. } \\
1 \text { nivalis ........ }\end{array}$ & $\begin{array}{l}\text { ANDREAA. } \\
\text { snowy .......... }\end{array}$ & Rocks..... & $6-8$ & P. & 2507 \\
\hline
\end{tabular}


SPECIFIC CHARACTER.

516. LESKEA.

Il L. oblong, apiculatc, cntire, nerveless : caps. ovate, erect: lid rostrate. For 9 other species, see Hypmum, Eng. Bot. and Grev. Crupt. Fl.

\section{IIYPNUN.}

11 L. ovate-lanceolate, acuminate, entire, the nerve reaching nearly to the summit: caps. oblong, drooping: lid conical.

For 52 other species, see ling. Bot. and Grev. Cryp. Fl.

Tribe III. VAGINULATI SCHISTOCARPI.

\section{ANDREAA.}

1 Stcms slightly branched: L. lanceolate, loosely imbricated, somewhat sickle-shaped: perichoetial similar to caulinc.

For 3 other species, see Muse. Brit. 



\section{CLASS XXIV. CRYPTOGAMIA.}

\section{ORDER III. HEPATICA.}

Herb frondose; not submersed. Capsules without a lid.

19 IUxgramaxia. Caps. 4-valved, supported on a peduncle longer than the calyx. V'ulves free.

20 Marchantia. Caps. on the under surface of a common peltate, pedunculate receptacle. Anthers imbedded in the disk of distinct pcltate pedunculate, or sessile receptacles.

$\therefore 21$ Riccia. Caps. sphcrical, immersed in the frond (not opening,) crowned with the style.

22 Axthoceros. Caps. stalked, linear, 2-valved, with a central axis to which the seeds are attachod.

¿23 Targioxia. Cups. globose, nearly sessile, included in a perianth of 2 concarc valves; opening irregularly at the extremity, and filled with spiral filaments.

¿24 Spuracarpus. Caps. minute, splierical, seated upon obpyriform receptacles; and filled with minutc seeds unmixed with filaments. 
CLASS XXIV. CRYP'TOGAMIA. ORDER HII. HEPATICA.

JUNGERMANNIA. JUNGERMANNIA.

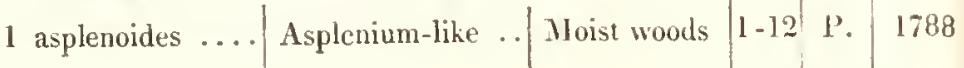

MARCHANTIA.

MARCHANTIA.

1 polymorpha.... variable . .....

LINNEAN NAMLS.

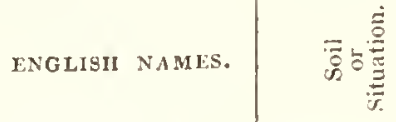

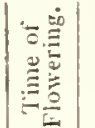

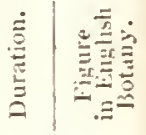

ANTHOCEROS. ANTHOCEROS.

1 punctatus...... dotted........ Damp places

\section{TARGIONIA. TARGIONIA.}

1 hypophylla .... flat-Icaved .....

SPII EROCARPUS. SPIIEROCARPUS.

1 terrestris ..... 
LASS XXIV. CRYPTOGAMIA. ORDER IH. HIPATICA.

\author{
SPEEIFIC CHARACTER.
}

\title{
519. JUNGERMANNIA.
}

I1 L. obovate, ciliate, toothed, somewhat reeurved: fruit terminal and lateral : ealyptra oblong, compressed, oblique.

Fur 80 other species, see Eng. Bot. and Hook. Jung.

\section{MARCHANTIA.}

1 Recept. of the capsule 10-eleft; that of the anthers peduneulate. For 3 other species, see Eng. But. 503. 504. 2545.

\section{RICCIA.}

1 Frond small, oblong, somewhat divided: segments 2-lobed at the end: flesliy, glaucous, lotted.

For 3 other species, see Eng. Bot. and Dick. Plunt. Cryp.

\section{ANTHOCEROS.}

1 Fronds multifid, lobed, sinuated: eaps. subulate, half bifid.

For 2 other species, see Ling. Bot. and Dill. Mus.

\section{TARGIONIA.}

1 Frond flat, imbricated, lobed; lobes rounded, retuse.

\section{SPHEROCARPUS.}

i Frond simple, ovate: eaps. pyriform, elustered at the base of the frond. 


\section{CLASS XXIV. CRYPTOGAMIA.}

\section{ORDER IV. ALGA.}

Aquatic plants, submersed. Caps. or tubercles variously situitcd: or seeds naked, or immersed in the frond.

\section{'Tribe 1. DIATOMA.}

Bodies of various forms, flat, crystalline, separating into frugments.

525 Achinantues. Frond stalked, vexilliform.

526 Diatona. Filaments jointcd, transparent, rigid, simple, united in pairs longitudinally; at length separating into urticulations cohering by their alternate angles.

527 Fragillaria. Filaments jointcd, simple, gelatinous, compressed, fragile, separating at the joints.

528 Meloseira. Filaments jointed, contracted at the joints, fragile, and easily separating.

529 Desmidun. Filaments transversely striated, mucous, ficxible, green, half separated into articulitions, and in that state pinnatifid.

530 Scnizonama. Filaments bead-like, composed of narrow cohering filaments, inclosing elliptical seeds, into which they are finally dissolved.

\section{TrIBE Il. NOSTOCIIINA.}

Individuals mumerous, globular or filiform, suspended in a gelatine of" a definite form.

531 Palmella. Small gelatinous plants, filled witl solitary seeds unnixed with fituments.

532 Ecmnelza. A rounded gelatine crowned with elliptical, radiant, corpuscles.

533 Ancroniduri. A spongy, fleshy, lobed frond filled with seeds.

534 Nostoc. Composed of curved, moniliform, simple filaments, lying irregularly in a gelatinous nidus.

535 Conrneproma. A gelatinous, roundish, puckered fromul, filled with jointed filements hearing club-shaperl processes.

536 Ruvelaria. A gclatinous, subglobose fromd, filled with filuments radiating from a common centre, contimons, planed on a gloluce, and markest with annulations insiele. 
17 Ch.торния. Elongated or globose, gelatinous: substance composed of branched, articulated filaments.

38 Scrthymexia. A corlaceous stratum, composed of fibres and seeds mingled together.

\section{TRIBE III. CONFERVOIDEA.}

Filaments jointed externally or internally, separate, and not combined in any definile form.

39 Brssocladiun. Filuments like cobwebs, scattered externally with seed-ressels.

10 Mrcisena. Filamenls membranous, opaque, tenacious, coloured.

11 Cinoolepus. Filaments rigid, nearly solid, opaque, crumbling into powder, uneven.

12 T'rexteronla. Filuments flcxible, coloured, bearing capsules generally proceeding from the last articulution, which is inflated.

143 Scrtoneis. Plant not gelatinous, coriaceous. Filaments short, forming dense tufts, internally beaded, or filled with annular transverse bodies.

H4 STIGONEMA. Filoments coriaceous, naked, continuous, marked inside with dots disposed in rings.

45 Protonema. Filaments very minute, somewhat jointed, rooting.

46 IYGrocrocis. Filuments cobweb-like, transparent, obsoletely articulated, floating in a shapeless gelatine, or in a coloured membrane.

47 Lepromisus. Filuments transparent, or slightly coloured, colweblike, obsoletely articulated, separate, erect, not entangled.

48 Mesoglon. Frond filiform, cylindrical, gelatinous, with compact, moniliform branches, radiating from a pith, and bearing capsules.

49 Batraciospermum. Frond filiform, gelatinous, sending out from the primary filament, moniliform, gemmiferous branches.

50 DrapanNaLDA. Filuments green, jointed, gelatinous: branches pencil-formed, fascicled. Fructifirution in a granular mass in the articulations of the main flaments.

51 Oscrllatoria. Gelatinous. Fillments simple, continuous, membranous, filled iuternally with transverse parallel strice.

.52 C.rotnnix. Filuments destitute of a mucous matrix, stiffish, straiglıt, notionless; with a continuous tule annulated inside. 
553 Lingrya. Filaments without a mucous matrix, freely floating, flexible, motionless, with a continuous tubc annulated inside.

554 Baxgra. Filaments capillary, mostly simple, tubular, continuous. Fructification composed of granules disposed in rcgular transverse series or strata.

555 ZYixisi. Filaments jointed, simple, gelatinous, compressed, fragile, scparating at the joints.

556 Movgeorna. Filaments articulated, connected like a net, with granules irregularly placed, and capsules attached to the angles of the meshes.

557 Hydnoncrion. Filaments articulated, connected like a net. $1 \%$ ticulations viviparous, including young individuals.

\$58 Conferva. Filuments uniform, jointed, membranous, simple or branclied, mostly green. Gromules scattered in thic articulations.

559 Burnochate. First filament articulated; sending out from the apex of the articulations, an accessory branchlet. Cupsules alternating with the accessory branchlcts.

560 Niteil... Filaments composed of a single tube, membranous, jointed, witl whorled branches. Organs of fructification two-fold and separate:- the first, mucules spirally striated, without bracteas, and not crowned; the second, coloured globules.

661 Cusm. Filumenls spirally striated, jointed, with whorled branches. Urgans of finctificulion two-fold, and close together: - the tirst, nucules spirally striated, bracteated, and crowned: the second, coloured globules.

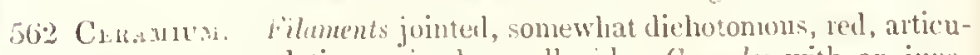
lations veined or pellucid. Capsules with an iurolucre of short brinchlets.

563 Cimfrmisti. Filuments jointed, branched, rose-red. Irticula(ii) as marked with a browel tube-like linc: the joints pellucid. Pedunculated rapsales ou the bramchlcis.

564 Cin 1.zosirons. Filaments obsoletely articulated, rosy, covered by axillary, asticulated, fruit-bearing branches, which either inclucle a globe of seids, or change to is lanceclate receptacle covered with bristles, among which the seeds nestle.

565 Ponssinosis. Filaments jointed, longitudinally striated, with intermal paratlel tubes. C'upsule's double, ovate; and granules in swollen branchlets. 
66 Rytıpnz£s. Frond flattened, distiehous, transvcrsely striated, bccoming blaek when dry, with incurved, withered scales. Fruit two-fold: the first, spherical capsules with pear-shaped sporidia; the second, lanecolate poels, with roundish sporidia.

167 Ecrocarpus. Filaments jointed, branched, dingy brown. Frnitlaneeolatc pods, or ovate capsules, solitary or raeemose.

.68 Sphacellaria. Filaments jointed, branched, olivaecous, distichous, or dichotonous: apex of the branches withered or crystalline, abrupt. Fruit-granules in the apcx; or cupsules.

69 Clabosteprus. Olivaceous. Main filuments opaque, not artieulated; branches jointed, mostly whorled with branchlets. Fructification-capsules.

\section{TRABE IV. ULVACEA.}

Frond membrunous, continnous, ubbular or flattened, never ribbed; green, or, very ravely, purple. Fruit-a heap of seeds, cither naked, or forming scattered grouules.

570 Vaucrerta. Filaments diehotomous, or irregularly brancherl, rigid. Fructification-a granulated mass within the frond, and external dark vesieles variously situated.

571 Conius. Frond spongy, of a determinate figure, formed of filtments closely paeked, which are tubular and continuous, and coloured by a granular green powder.

:572 Bryorsis. Root smalt, buckler-shaped. Filuments tubułar, eontinuous, aggregate, branehed, pinnate, or imbrieated with branchlets. Fructification-a dark internal granular mass.

573 solesia. Frond tubular, inembranous, with a striated, areolated surfaee. Spuvidia very minute and compact.

574 ULr.s. Romt buekler-shaped. Frond plane rilless, fan-shaped, or wodge-shaped, or linear and dichotomous. Fructificution naked, immersed, grunules distributed in 4's, throughout the frond.

575 Ioninyus. Fromel hat, purple, with the membrane of equal texture. Frinit two-fold:-the first, masses of oval sporidia collected in a disorderly manncr; the second, two parallel lines marked on eateh side by a globule. 


\section{Tribe V. FLoRIJ)E.}

Frond corioceous or membrumous, flat or filiform, continuous, purple or pink. Spuridia purple, enclosed in capsules, or clustered in masses. 576 Pouxides. Frond filiform, fastigiate, cartilaginous, softish, composed of radiating fibres. Fruit-spongy warts composed of fibres supporting sporidiu.

577 Pтицота. Root buckler-shaped. Frouds compressed or flat, pinnate. Fructificotion-a cluster of naked gramules surrounded by a linear cleft involucre.

578 Rhonomela. Frond either flat or foliaceous, and ribbed or filiform. Fruit two-fold:-the first, lomenta filled longitudinally with globules; the second, capsules containing pear-formed, sessile sporidia.

579 Cuovinria. Fromd continuous, gelatinoso-cartilaginous. Fructification double: raked granules immersed in the substance of the branchlets and external tubercles.

580 Sphrirococcus. Koot buckler-shaped. Frond membranous or cartilaginous. Fructificution uniform: tubercles or capsules.

581 HaLvien1s. Froud flat or tubular, somewhat membranous. Fruit -dot-like tubcreles half immersed in the lamina of the frond.

582 Bonnemasonic. Frond filiform, compressed, pectinate, ciliate. Fruit-capsules with pear-shaped sporidia, fastened together like a chain.

583 DeLissenia. Liot buckler-shaped. Frond tlat, membranous, with or without ribs. Fructification double:-tubercles and clusters of naked and immersed gramules.

\section{THBE VI. FUCOIDEA.}

firond coriacens or rarely membrunous, continuous, flat or filiform. wive-grren. Sporidiu black, enchsed in capsules, which are either mate, surmundal by a limusparent border, and nestling in a peculiar receptucle: or pear-shaped and inmersed in the fromd.

584 Lranes. Fronul filiform, uncen, tubular. Chains of spmilia adluering to the inner surface of the filament, pencilled, monilitorm.

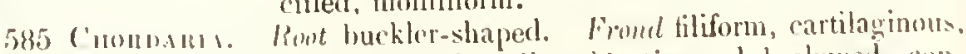
olive-cotomed, fimelificulion-club-shitped, concentric filanents, constituting the whole fromd. 
ORDER IV. ALGAT.

ACHIN ANTHLS.

1 longipes

.

ACHNANTHES.

$$
\text { DIATOMA. }
$$

1 flocculosum

FRAGILLARIA.

1 pectinalis........

long-stalked

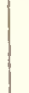

ENGLISI NAMLS.

floc

DIATOMA.

floccose

FRAGILLARIA.

silvery...........

On wat. plants

MLLOSEIRA.

1 nummuloides ....

necklace

Salt marshes

DISSMIDIUM.

1 Swartzii .......

pinnatifid

\begin{tabular}{ll|l|l} 
Still waters . & $6-8$ & $* 2464$
\end{tabular}

SCHIZONEMA.

SCIHZONTAMA.

1 smithii.

Smith's

Sea coast...

$5-8 * 2101$

P'ALM FLLA.

1 protuberans

lobed

Rocks.... 3-11,*2583

BCIIINELA.

\section{ECHINELLA}

1 arliculata....... jointed

Jake...... $0.7 * 1378$

A.CYONHDHUI.

ALCYONIJHAH.

1 diaphanmu

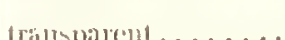

trürsparent....... Ocean ..... (6-8 
TRIiz 1. DIATONEA.

525. ACIINANTIIES.

Articulations with one dot: stem long. Conferva. Eng. Bot.

526. DIATOMIA.

Filaments striated: articulations nearly equal in diameter with parallel strix. Conferva. Eng. Bot.-For 4 other species, see ling. Bot.

\section{FRAGILLARIA.}

Filaments tapering, very rigid, with parallel, transverse, dense striæ. Conferva. Eng. Bot.

\section{MELOSEIRA.}

Filaments unequal, containing nearly circular moniliform globules in rows.

\section{DESMIDIUM.}

Filaments finally pinnatifid, traversed by a longitudinal green streak: articulations 2-toothed. Conferra. Eur. Bot.

\section{SCHYZONEMA.}

Filaments somewhat branched, tufted, acute: granules parallel, clustered. Conferva. Eng. Bot.

For oiher speeies, see Grev. Crupt. Bic.

Tribe II. NOSTOCHIN A.

531. PALMELLA.

1 Frond thick, angular, lobed: granules elliptical. Ulou. Fing. Bot. For other species, see Eng. Bot. \&.c.

\section{ECHINELLA.}

1 Corpusclcs radiant, lanceolate, jointed. Conferra. Eng. Bot.

\section{ALCYONIDIUM.}

In Pranches elongated. Illoa. Fing. Bot. For another speries, see ling. Bot. 1626. 
CLASS XXIV. CRYPTOGAMIA.

ORDER 1V. ALGA.

\section{(2...}

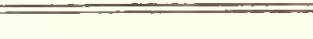

LINNEAN NAMES.

NOSTOC.

1 commune. CORYNEPHORA.

1 marina.........

RIVULARIA.

1 atra.

\section{CHETOPHORA.}

1 tuberculosa ......

\section{SCYTIIYMENIA.}

1 rupestris

BYSSOCLADIUM.

1 fenestrale........

13

13YSSOCLADIUM.

window

\section{MYCINEMA.}

1 arachnoideum ....

MYCINEMA.

cobweb

\section{CHROOLEPUS.}

1 ebeneus.......... ebony ............ TRENTEPOILLA. TRENTEPOHLIA.

1 purpurea ........ purple.

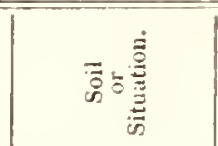

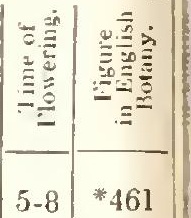

Damp places

\begin{tabular}{ll|l|l} 
Sea shore $\ldots$ & 8 & $* 1956$
\end{tabular}

$10 \quad 1798$

Ditches

9

$* 2366$

Rocks..... 6-8

2194

On windows

(1)

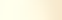
.

Dead trees .. $1-12$

\begin{tabular}{ll|l|l} 
Rocks...... & $1-12$ & 702
\end{tabular}

Rucks.Den co. $1-12 \mid * 192$
Sea plants .. 
SPEEIFIC CHARACTER.

534. NOSTOC.

1 Frond expanded, deformed, plaited, wavy. Tremella. Eng. Bot.

\section{CORYNEPIIORA.}

1 Rimularia tuberifornis. Eng. Bot.

\section{RIVULARIA.}

1 Frond hemispherieal, hard : filaments dense, branehed by apposition. For 2 other species, see Eng. Bot.

\section{CHATOPHORA.}

1 Frond tubercular, hollow: filaments distributed in many little orbs. Rivularia. Eng. Bot.

\section{SCYTHYMENIA.}

1 The only speeies.

\section{Tribe III. CONFERVOIDEA.}

\section{BYSSOCLADIUM.}

1 Filaments appressed, very minute, short, radiant, eobweb-like, branehed, sinuous, wavy.

\section{MIYCINEMA.}

1 Filaments thin, entangled in a cobweb-like membrane: branehes scattered: articulations of various lengths. For 4 nther species, see Dill. Conferea.

\section{CIROOLEPUS.}

I Filaments tufted, branched, erect, rigid, olotuse, somewhat cartilaginous: artieulations as broad as long. Byssus nigra. Eng. Bol.

\section{TRFNTEPOHLTA.}

1 Filaments diehotomous, tufted, minute, entangled : artieulations about twice as long as broad. Byssus. King. Kot.

$$
\text { For } 2 \text { other species, see ling. Bot. }
$$


CLASS XXIV. CRYPTOGAMIA.

ORDER 1V. ALG L.

STIGONEMIA.

1 atrovirens........

1 typhloderma......

Gum Arabic .

PROTONEMA.

1 cryptarum

STIGONEMA. dark green ........

Rocks ..... 6-8

PROTONEMA. .

SCYTONENA. tufted .

\begin{tabular}{|c|c|c|}
\hline $\begin{array}{c}\text { Situation } \\
\text { or } \\
\text { Place of Growth. }\end{array}$ & 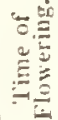 & 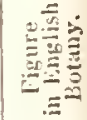 \\
\hline Ocean ...... & 10 & ${ }^{*} 1700$ \\
\hline
\end{tabular}

1 comoides

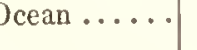

Caverns ....

1 lacteus

\section{LEPTONITUS.}

In sol. Gum

Arabic

\section{LEPTONITUS.}

milky

Pools

MESOGLOIA.

1 Iludsoni

\section{MESOGLOIA.}

Hudson's

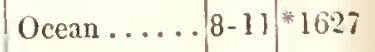

BATRACHOSPERMUN. BATRACHOSPERAUM.

1 vagum .......... turfy

$\beta$ tenuissimum......

very slender.

i

Ditches

DRAPARNALDIA.

DRAPARNALDIA

1 plumosa ........ feathery

Rivulets ....

$6-8 \div 2087$

OSCILLATORIA.

OSCILLATORIA.

I tenuissima very fine.. 


\section{SCYTONEMA.}

1 Tuft loose: filaments flcxuose: lranches solitary, remote, ascending. Conferta. Fng. Bot.

For other species, see Eng. Bot.

\section{STTGONEMA.}

1 Filaments rigid, branched: branches slender: granules disposed in rings.

\section{PROTONEMA.}

1 Filaments dichotomous: branches divaricating, acuminate: articulations tlrice as long as broad. Comferva. Eng. Bot.

For 6 other species, see Eng. Bot.

\section{HYGROCROCIS.}

I Filaments somewhat branchcd, entangled in an olive-green pellicle: articulations as broad as long.-Fm nther species, see Dillw. Con.

\section{LEPTOMITUS.}

1 Filaments at cvery joint, branched, and clustered in a shapeless, gelatinous mass: articulations very long.

\section{MESOGLOIA.}

l Frond virgate, with all the branches divaricating. Ulia rubro. Eng. Bot.

\section{BA'RACHOSPERMUM.}

1 Frond dicholomous, cylindrical, equal: branches thickencd at the cnd. $\beta$ Frond setaceous, minute. Conjerva atra. ling. Bot.

\section{I)RAPARNALDIA.}

1 Pencils of branches lanceolate, acute, crect. Conferta lubrica. Eng. Bot.-For 2 wher species, see Eing. Bot. and Dillw. Cumf:

\section{OSCILLATORIA.}

1 Filaments transparent, very fine, tufted, entangled in ncarly parallel lines. Comfertr. ling. Bot.

For several uther species, see Eng. Bot. and Dillw. Couf. 
CLASS XXIV. CRYPTOGAMIA.

ORDER IV. ALG.T.

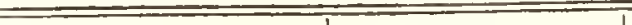

LINNEAN NAMES.

ENGLISH NAMES.

CALOTHRIX.

Situation
or
Place ot Growth.

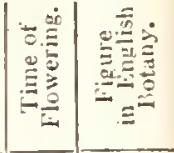

1 nivea snowy .............

1 muralis wall

LYNGBYA.

LYNGBYA.

B $\Lambda$ NGI.

I3 ANGIA.
1 atropurpurea

ZIGNEMA.

dark purple

ZYGNEMA.

1 crueiatum

1 utrieulatum CONFERI

1 rivularis bladdery riv

B Anglica

English

I setigera - setigerous

1 graeilis. slender

CHAR.
MOUGFOTIA.

\section{MOUGEOTIA,}

1 creruleseens ......

Ditcles ...

$$
7
$$

Sulph. springs I -12.

$\begin{array}{llll}\text { Damp earth } & 1-12 & 1554\end{array}$

Marine rocks

$5-8$ $6-8 * 2463$

HYDRODICTYON.

II DRODICTYON IRi

CONFERT $\Lambda$.

Rivers..... 4-8

1654

Ditches

13ULBOCIIETF.

\section{NITELLA.} 1'ools

Lakes. Rivers 8-11

1687

4-8

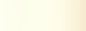




\section{SPECIFIC CHARACTER.}

\section{CALOTHRIX.}

1 Filaments very fine, rigid, snow-white, packed in a dull yellow tuft. For 6 other species, see Eng. Bot. and Dillw. Conj".

\section{LYNGBYA.}

1 Filaments stittish, curved, wavy, thickish, with lax rings.

\section{BANGIA.}

1 Filaments dark purple, straight: bands 5-dotted.

\section{5. 'ZYGNEMA.}

1 Articulations twiec as long as broad : stellæ roundish : fruit spherical. Conferva. Eng. Bot. - For 7 other species, see Eng. Bot. and Dillw. Conf.

\section{MOUGEOTIA.}

1 Filaments purple-bluc: seed-vessels of the crosses of the filaments grcen. Cunferia. Eng. Bot.-For another species, see Dillw. Conf.

\section{IIYDRODICTYON.}

1 Spots 5-cornered. Conferva reticulata. Eng. Bot.

\section{CONFERVA.}

1 Filaments simple, capillary, very long, straight, equal: artieulations grain-bearing, 2 or 4 times as long as broad; shining when dry. $\beta$ Aituculations half as long as broad.

For many other species, see Eng. Bot. and Dillw. Conf:

\section{BULBOCHUE'L.}

1 The only specics.

\section{NI'TELLA.}

I Stem sleuder, long: brunehes aeute, forked: fruit solitary. Churu. Eng. Bot.-For 4 other species, see London's Ercy. Plunts.

\section{CHARA.}

1 stem twisted, furrowed, strigose: striga reflexed: bract. aculeate. Fiv" whother species, see Eng. Bot. 336. 
CLASS XXIV. CRYPTOGAMIA.

ORDER IV. ALGA

I tetragonum ......

\section{CERAMIUM.}

ENGLISH NAAMES.

LINNEAN NAMES.

CERAMIUN. square..........

564

566

\section{GRIFEITHSIA.}

GRIFFITHSIA.

1 multifida....... multifid

CHETOSPORA.

CHATOSPORA.

1 Viggii .

Wigg's

POLYSIPHONIA.

1 parasitica.

POLYSIPHONIA. parasitical ........

Sea shore

\begin{tabular}{|c|c|}
\hline & 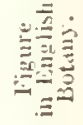 \\
\hline & 1690 \\
\hline
\end{tabular}

Ocean..... 6-8

1690

Sea shore 7

* 1816

.

RYTIPIILAA.

1 tinctoria

RY'IIPHLAA. dyer's

Ocean

On Fuci...

ECTOCARPUS.

1 siliculosus

\section{ECTOCARPUS.}

podded Oce

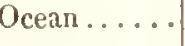

\section{SPHACELLARIA.}

SPHACELLARIA.

1 racemosa racemose Sea shore .. 
SPECIFIC CHARACTER.

\section{CERAMIUM.}

1 Filaments branched, virgate: primary articulations twice as long as broad.

Fir 20 other species, see Eng. Bot. and Dillw. Conft.

\section{GRIFFITHSI $\Lambda$.}

1 Filaments branched: branchlets subternate, distant, short, multifid: articulations much longer than broad. Conferva. Lng. Bot. For 4 other species, see Eng. Bot.

\section{CHETOSPORA.}

1 Fucus. Eng. Bot.

\section{POLYSIPHONIA.}

1 Filanents bipinnate, veiny, rigid: pinne and pinnules alternate: articulations rather short.

For several other species, see Eng. Bot. and Dillw. Conf.

\section{RYTIPHLAA.}

1 Frond somewhat cartilaginous, compressed, transversely bipinnate: pinnules in fruit incurved.

\section{ECTOCARPUS.}

1 Filaments nearly separate: branches ercct, subulate; articulations rather long than broad: pods linear-subulate.

For 3 other species, seg Eng. Bot. and Dillw. Conf.

\section{SPHACELLARLA.}

1 Filaments twice or thrice dichotomous: articulations as long as broad: tubercles ovate, raccmosc, on branched peduncles.

For 5 other species, see Eng. Bot. and Dillw. Conf.

\section{CLADOSTEPIIUS.}

1 Seta simple, denscly imbricated. Conferva. Eng. Bot. For another species, see Eng. Bot. 1718. 


\begin{tabular}{|c|c|c|c|c|c|}
\hline & \multicolumn{2}{|c|}{ CLASS XXIV. CRYPTOGAMIA. } & \multicolumn{3}{|c|}{ OHDER IV. ALGAL. } \\
\hline & LINNEAN NAMEE. & EAGLISH NANES. & $\begin{array}{c}\text { Situation } \\
\text { Place of Growth. }\end{array}$ & 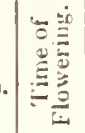 & 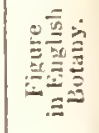 \\
\hline 570 & $\begin{array}{l}\text { VAUCHERIA. } \\
1 \text { dichotoma .... }\end{array}$ & $\begin{array}{c}\text { VAUCHERIA. } \\
\text { dichotomous ..... }\end{array}$ & Ditches .... & $5-8$ & $* 932$ \\
\hline 571 & $\begin{array}{c}\text { CODIUM. } \\
\text { I tomentosum... }\end{array}$ & $\begin{array}{c}\text { CODIUN. } \\
\text { downy........... }\end{array}$ & Ocean... & 6 & $* 712$ \\
\hline 572 & $\begin{array}{l}\text { BRYOPSIS. } \\
1 \text { plumosa ..... }\end{array}$ & $\begin{array}{c}\text { BHYOHSIs. } \\
\text { feathery .......... }\end{array}$ & Ocean. & 9 & 23375 \\
\hline 573 & $\begin{array}{c}\text { SOLENIA. } \\
1 \text { compressa } \ldots\end{array}$ & $\begin{array}{c}\text { SOLENIA. } \\
\text { compressed........ }\end{array}$ & Ocean. & $5-8$ & $* 1739$ \\
\hline 574 & $\begin{array}{c}\text { ULVA. } \\
1 \text { lactuca....... }\end{array}$ & $\begin{array}{c}\text { ULVA. } \\
\text { Green laver } \ldots . . .\end{array}$ & Ocean... & $.5-10$ & 1551 \\
\hline 575 & $\begin{array}{c}\text { PORPHYKA. } \\
\text { 1 laciniata ......... }\end{array}$ & $\begin{array}{c}\text { PORPHYRA. } \\
\text { torn............... }\end{array}$ & Sea shore & $6-8$ & o..... \\
\hline 576 & $\begin{array}{c}\text { POLYIDES. } \\
1 \text { lumbricalis ..... }\end{array}$ & $\begin{array}{c}\text { POLYIDES. } \\
\text { worm-like } . . . . . .\end{array}$ & Ocean...... & 11 & $* 1738$ \\
\hline 577 & $\begin{array}{c}\text { PTILO'TA. } \\
1 \text { plumosa ..... }\end{array}$ & $\begin{array}{c}\text { P'IILOTA. } \\
\text { feathery ......... }\end{array}$ & Sea shore & & \\
\hline 578 & $\begin{array}{l}\text { RHODOMELA. } \\
1 \text { dentata......... }\end{array}$ & $\begin{array}{l}\text { RIIODOMELA. } \\
\text { toothed ......... }\end{array}$ & Sea shore & $.6-3$ & $* 1241$ \\
\hline
\end{tabular}


SIECIFIC CHARACTER.

\section{THвE IV. ULVACEA.}

570. VAUCHERIA.

1 Filaments setaceous, diehotomous, fastigiate: eaps. globose, sessile, sulitary. Conferva. Eng. Bot.

For other species, see Eng. Bot. and Dillw. Conf.

\section{CODIUM.}

7 Frond diehotomous, fastigiate, eylindrieal. Fucus. Eng. Bot. For another species, see Eng. Bot. 2183.

\section{BRYOPSIS.}

1 Filaments branehed, naked below, pinnate in the middle: branehlets opposite, nearly simple, approximating.

\section{SOLENIA.}

1 Frond tubular, lined, latticed, branehed, filiform, eompressed : branches simple, tapering at the base.

\section{ULVA.}

1 Frond obovate, or laneeolate, flat, wavy, laeiniate-erisp. For 2 other species, see Lou. Ency. 1 l.

\section{PORPHYRA.}

1 Frond flat, with numerous dilated segments.

$$
\text { Tribe V. FLORIDE } A \text {. }
$$$$
\text { 576. POLYIDES. }
$$

1 Fucus rotund. Eng. Bot.

\section{PTILOTA.}

1 Frond pinnate; pinnules peetinate, cut. Fucus. Eng. Bot.

\section{RIIODOMELA.}

1 Frond Alat, obsoletely ribbed, alternately bipinnatifid: pinna linearwedge-shaded : pinnules eut: caps. pitcher-shaped. Fucus. Engr. Bot.-For 4 ather species, see Eng. Bot. 
ORDER IV. ALGA.

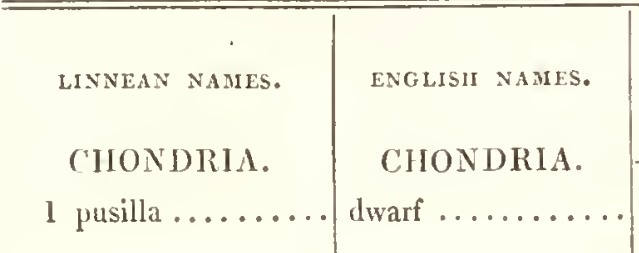

SPIIAROCOCCUS. SPIIEROCOCCUS

1 ruber red.

Marine rocks

Situation
or
Place of Growth.

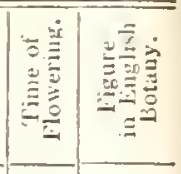

BONNEMAISONIA. BONNEMAISONIA.

\section{1 asparagoides ..}

1 sanguinea........

584

585

586

583

sanglinea........

1 fluviatilis ........

\section{CHORDARIA.}

1 flagelliformis .... SCYTOSIPIION. 1 filum

1 edulis ........

IIAL YMENIA.

Ocean ...... 11-2*1053

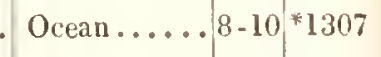

$$
\begin{gathered}
\text { asparagus-like ... } \\
\text { DELESSERIA. }
\end{gathered}
$$

blood-coloured ....

Sea shore ..7.1 I

*571

LENANIA.
fluviatile .........

On stones in rivers .... 6-8

\section{CHORDARIA.} tlagelliform ........ Ocean.... 6-8*1222 SCYTOSIPLON. . cord ........... \begin{tabular}{|l|l|l} 
Ocean ..... & $6-8$ & $\ldots$. \\
Sea shore .. & $6-8$ & $* 545$
\end{tabular} SPOROCHNUS. stalked

1 pedunculatus

\section{SPOROCINUS.}

prominlatus 
SIECIFIC CHARACTER.

579. CHONDRIA.

1 Frond compressed, filiform, somewhat contracted herc and there: fructification either minute tubercles, or scattcred sced-vesscls in small branches. - For 8 other species, see Eng. Bot.

\section{SPHEROCOCCUS.}

1 Stcm scarcely any: laminæ chained, obsoletely ribbed, wedge-shaped, 2 -forked or lanceolate: caps. rugose, sessile in the disk of the frond. Fucus. Eng.Bot._For many other species, sce Lou. Ency.Pl.

\section{HALYIIENIA.}

1 Frond fleshy, flat, simple, wedge-shaped; tapered at the base into a footstalk; rounded at the cnd. Fucus. Eng. Bot. For 6 other species, see Lou. Ency. Pl.

\section{BONNEMAISONIA.}

1 Frond filiform, compressed, much branched: branchlets setaceous, distichous, simplc, pectinate on each side. Fucus. Eng. Bot.

\section{DELESSERIA.}

1 Stem distinct: L. ovate, stalked, entire, ribbed: nerves transverse parallel. Fucus. Eng. Bot.

\section{Tribe VI. FUCOIDE $\boldsymbol{E}$.}

\section{LEMANIA.}

1 Filaments sinple papillose; papillæ usually ternate: articulations 5 times as long as broad.

\section{CHORDARIA.}

1 Frond much branched: branchlets virgate, somewhat distichous, spreading at the base. Fucus. Eng. Bot.

\section{SCYTOSIPHON.}

1 Frond quite simplc.

\section{SPOROCHNUS.}

1 liccept. clliptical, lateral, as long as the peduncle. Fincus. Eng. But. For wher species, see Eug. Botound Tumer's l'uci. 


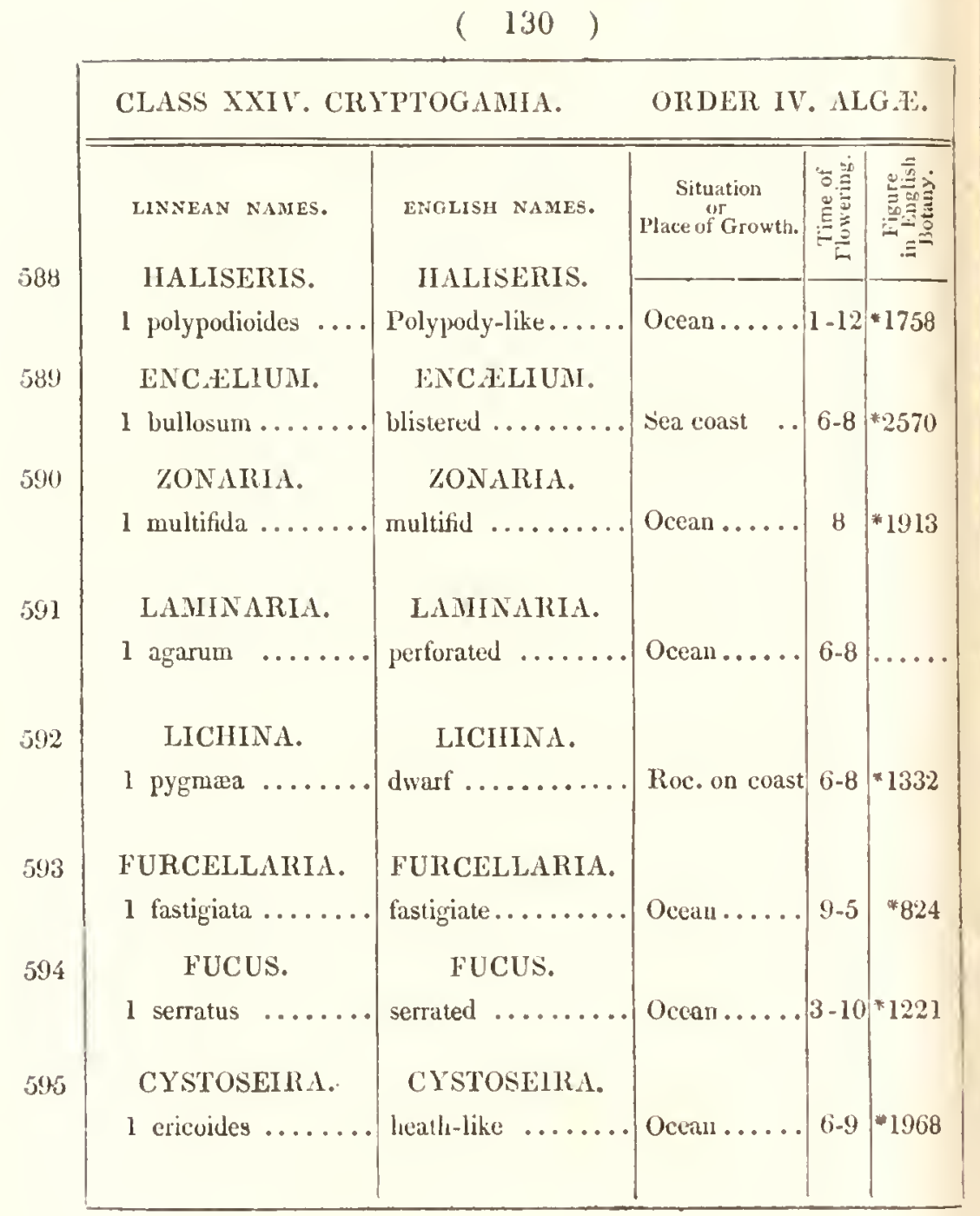


588. HALISERIS.

1 Frond linear, dichotomous, entire; sori heaped about the ribs. Fucus Eng. Bot. 589. ENC ALIUM.

1 Frond inflatcd, club-shaped. Ulva. Eng. Bot.

$$
\text { 590. ZONARIA. }
$$

I Frond diehotomous, entire: segments long, slender, acutc. Illea. Eng. But.-For 2 other species, see Eng. Bot.

\section{LAMINARIA.}

1 Stalk running through the lamina, which is riddled with holcs. For 5 other species, see Eng. Bot. and Turn. Fuci.

\section{LICHINA.}

1 Frond flat, with spherical tubcreles. Fucus. Eng. Bot. For unother species, see Eng. Bot. 2575.

\section{FURCELLARIA.}

1 F. lumbricalis. Eng. Bot.

\section{FUCUS.}

I Frond diehotomous, ribbed, serrated : reeept. solitary, flat, serrated. For other species, see Eng. Bot.

\section{CYSTOSEIRA.}

l L. densely spiny all over: vcsielcs elliptical, somewhat tem. crown, solitary: recept. warty, from the inflated basc of the spines. Fucus. Eng. Bot.-For other species, see ling. Bot. 


\section{CLASS XXIV. CRYPTOGAMIA.}

\section{ORIER V. LICHENES.}

Fruclification in shields, tubercles or warts, in whose disk the seeds are lodged.

\section{TRIBE I. IDIOTHALAMI.}

Receptacle composed of a substance different from the frond, and discoloured.

* Receptacles wilhout a border, or nucleus.

596 Spicoma. Plant crustaceous, spreading, flat, adnate, uniform. Fructification composed of minute bodies, collected iuto a shapeless mass.

597 Solorina. Plant leafy, coriaceous, veiny bencath. Recept. adnate, roundish, covered with a membrane, aud eontaining a solid cellular pareneliyma.

** Receplacles bordered.

598 Lecidea. Plant various, crustaceous, spreading, uniform or leafy. Recept. shicld-shaped, sessile, surrounded by a cartilaginous membrane, disk flat.

599 Calicium. Plant crustaceous, flat, adnate, uniform. Recept. cup-shaped, sessile or stalked, cartilaginous, containing a powdery mass, either flat or convex.

600 Grrophora. Plant leafy, eoriaceous or eartilaginous, peitate, mostly monophyllous, free beneatl. Recept, roundish, sessile, eoneentrieally plaited, eovered with a blackish membrane.

601 Endocanpon. Plant crustaecous, adnate, of some determinate figure; or leafy and peltate. Frnctification globose, enclosed in the substance of the plant : composed of a nucleus covered with a thin membrane. fumished with a slightly prominent orifice. 


\section{Tribe II. CANOTHALAMI.}

Receptacle in part composed of the substance of the plant.

* Recept. wart-like.

302 Themotrfas. I'lant clustaceous, eartilaginous, flat, adnate, uniform. Recept. bordered, furnishcd with a wide pore, and eontaining a nucleus within a double eovering.

003 Proxula. Plant crustaceous, adnate, uniform. Fructificalion, a cellular globular nucleus, with a thiek cartilaginous blaek covering.

604 Tariolaria. Plant crustaceous, flat, spreading, alnate, uniform. Recept. formed from the erust, bordcred, white, inelosing a naked mucleus.

* Recept. shield-like, subsessile, the disk differently coloured from the border, which is formed from the crust.

605 Ureeolaria. Plant crustaceous, sprcading, adnate, uniform; disk of the recept. coneave, eoloured, immersed in the crust.

606 Leensora. Plant erustaeeous, spreading, adnate, flat, uniform. Rerept. thick, sessile, the disk plano-eonvex, coloured: border thickish, somewhat free.

607 Parmelia. Plant leafy, coriaceo-mcmbranaceous, spreading, appresscd, lobed and stellate, variously divided, fibrous bencatl. Recept. altached by the centre: disk concave, colourcd.

608 Borrera. Plant cartilaginous, jagged into branehes, whieh are channelled beneath, and fringed. Disk of the recept. coloured.

609 Cetraria. Plant cartilagino-membranaceous, lobed, smooth on both sides. Recept. obliquely attached to the margin; disk coloured, plano-eoncavc; bordcr inflexed.

610 Sтетл. Plant leafy, coniaeeo-cartilaginous, lobed, downy beneath, scattercd with hollow spots. Recept. fixed by the centre; disk flat, coloured.

611 Peltidea. Plant lcafy, eoriaceous, spreading, lobed, with downy veins beneath. Recept. elosely attaehed to the upper margin of the frond; bordcr very thin.

612 Nepinoma. Ilant leafy, corlaceous, membranaceous, lobed; scparate, and naked or woolly, beneath. Recept. formed of the asecnding lengthened lobes of the thallus, surrounded by an elevated, inflexed margin. 
613 Roccelds. Plant coriaceous, cartilaginous, branched, laciniated, cylindrical or flat, erect or pendulous, woolly inside. Recept. thick, growing into the thallus; disk planoconvex, surrounded by an elevated, sessilc margin, which contains a compact black powdery mass.

614 Everna. Plant branched, laciniate, angular or compressed, pendulous or somcwhat erect, with a central filament within. Recept. sessile; disk concave, coloured.

** Recept. in little heads or globules, terminating the branches, $m$ the stalk-like processes of the frond; or scattered, sessile, and emarginate.

615 Семомгсе. Plant somewhat cartilaginous, leafy, laciniate, rarely uniform. Recept. convex, attached by the edge, to hollow stalk-like processes, and bearing thick coloured masses of fructification.

616 Вæомусеs. Plant crustaceous, sprcading, flat, adnate. Recept. on short, soft, solid stalk-like proccsses; solid, convex, emarginate, reflexed at the margin, colourcl.

617 IsIDIUm. Plant crustaceous, flat, spreading, adnate, uniform. Recept. on very short stalk-like processes, circular, convex, solid, terminal, disk inclosed by a border, formed from the process which supports it.

618 Srenfocaulon. Plant shrubby, cartilaginous, branched. Recept. turbinate, solid, sessile, flat above, at length subglobose, with a border formed from the frond.

619 Splifnopizonon. Plant cartilaginous, fibrous within, solid, shrubby, branched. Recept. nearly globular, terminal, fornncd of the frond, inclosing a black round seed-bearing ball, bursting by a torn orifice.

\section{Tribe III. HOMOTHALAMT.}

Recept. entirely formed of the sulstance of the frond, and of the sume colour.

620 Arfrtonra. Plant cartilaginous, branched; branches filiforn, hollow, and filbrous within; prostrate or pendulous. Recept. shield-like, thick, scssile, bordered.

621 Ramalna. Plant cartilaginous, fibrous, and nearly solid withim, branched, somcwhat shrubby. Recept. shicld-like, thick, somewhat stalked and peltate, Hat, bordcrel.

622 Connirularia. Plant cartilaginous, fibrous and nearly solid within, branehed, shrubby. Recept. eircular, tcrminal, obliquely peltate, at length convex, somewhat inflated; border dentatc. 
\$23 I'SEA. Plant branched, filiform, nostly pendulous, with central elastic filsres. Recept. circular, terminal, flat, peltate, often fringed at the edge.

324 Collesa. Plant sulgelatinous, homogenous, crustiform, leafy or somewhat branched, cartilaginous or membranaceous when dry. liecept. shield-likc, bordered; the disk sometimes diflering in colour, when dry.

\section{THIRE 11.ATHALAII.}

Lichenes of which the fructification is not known.

325 LEPraris. Plant crustaceo-pulverulent, spreading, adnate, uniforn1.

\section{THB: V. PALUDO-LICHENES.}

Recept. black, hornu, imbedded. Seeds in slender lubular cells, lying in a pulp, not spontaneously emitted.

326 OPEGR APHA. Plant crustaceous, flat, expanded, alnate, uniform. Recept. oblong, sessile, covered with a dark cariliaginous membrane, inclosing a solid parenchyma: lisk linear, edgerl on each side.

f527 Vrrrucaria. Plant crustaceous, flat, expanded, adnate, uniform. l'ecept. sumewhat globo:c, growing into the thitus, double coated: the outer coat thick and somewhat eartilaginous with a little pimple or perforation alsove : the inner fire and membranaceous. Kernet globular, cellular.

628 Porixa. Plant crustaceous, flat, expanded, adnate, uniform. liecept. wart -like, formed out of the thallus: fructification imbeddel within its substance. Kiernel roundish, cellular; in a thin envelope; with a coloured orifice. thicker at the surface of the wart.

629 Aптиома. Plant crustaceous, flat, expanded, adnate, uniform. kecept. innate, slapeless, covered with a dark, cartiIaginous membrane, and containing a solid uniform kernel.

630 Giraphs. Plant crustaceous, flat, expanderl, adnate, uniform. Recept. linear, immersed in the thallus; with a cartilaginous border surrounding the linear cellular kernel, but leaving it naked at the top and bottom. 
CLASS YXIV. CRYPTOGAMIA. OMDER V. LICHENLS.

SPILOMA.

SPILONA.

1 tumidulum

tumid

Bark of trees $1-12 \mid 2151$

1 crocea s.

SOLORINA.

SOLORINA. saffron coloured ....

Alp. hea.! .. $3.8 * 489$

1 lurida

DEA. (a) 


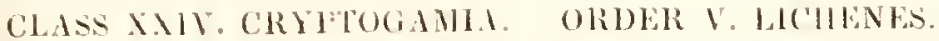

SPECIITC CIIILACTER.

TriBE 1. IDIOTHALAMI.

596. SPILUMA.

1 Crust somewhat eartilaginous, whitish: recept. tumid, oblong, red; finally brown.

For 11 other species, see Eng. Bot.

597. SOLORINA.

1 'Thallus green, lobed, reiny and saffiron-eoloured beneath: reeept. tumid, brown. Lichen. Eng. Bot.

\section{LECIDEA.}

1 Crust imbrieated, greenisl brown, with roundish erenate lobes, pale beneatl 1 : recept. flat, at length beeoming eonvex, black. Lichen, Eng. Bot.

For 65 other species, see Eng. Bot.

\section{CALICIUM.}

1 Crust filmy, very thin, whilish: reept. blaek, with blaek, prominent, loose powder.

For 16 other species, see Eng. But.

\section{GIROPHOR $\Lambda$.}

I Frond grcenish gray, lobed, fringed with blaek hairs: rceept. flattish, with coneentric, and plaited lines. Lichen prohuscideus, Ling. But. For 7 other species, see Eng. Bot.

\section{ENDOCARPON.}

I Thallus depressed, pcltate, flat, roundish, entire, grcenish yellow: orifices depressed, rcddish-brown. Lichen, Ling. Bot. For 9 other species, see Eng. Bot.

THibe II. CANOTHALAMI. 602. THELOTREMA.

1 Crust ercan-eoloured, polished: warts somewhat conical, within fleshcoloured, witl a thin infleeted cdge. Lichen inchusus, Eng. Bot. For 2 other splecies, see Loudon's Fincy. I't. 
CLASS XXIV. CRYP'TOGAMIA. ORDER V. LICHINES.

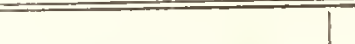

LINNEAN NAMES.

PYRENULA.

l nigrescens $\ldots . .$. .

ENGLISH NAMES.

\begin{tabular}{|c|c|c|}
\hline $\begin{array}{c}\text { Situation } \\
n \Gamma \\
\text { Place of Growth. }\end{array}$ & 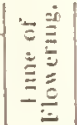 & 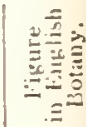 \\
\hline Rocks.. & $1-12$ & $* 1499$ \\
\hline
\end{tabular}

605

606

1 velata veiled

VARIOLARIA.

VARIOLARIA. blackish

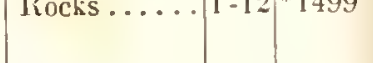

URCEOLARIA.

URCEOLARIA.

1 Acharii .

Acharian .......

Ash trees $8-10 \quad 2062$

Stones! .... 1 -12 1087

LECANORA.

cudbear .......

Rocks.....1-12*156

1 tartarea .

PARMELIA.

1 glomulifera warted.

Trunks of trees

BORRERA.

1 ciliaris f

BORRFRA. fringed $\ldots \ldots \ldots$ Trunks of trees $1-12 * 1352$

CETRARIA. Iceland-moss 
603. PYRENULA.

1 Crust somewhat tessellatcd, unequal, brownish black: warts spreading at the base, depressed, somewhat wrinkled. Ver. umbrinu. Eng. Bot.-For' 2 other species, see Eng. Bot. 2455. 2153.

\section{VARIOLARIA.}

1 Crust smooth, white, plaited in rays: warts polished, compressed, tumil: kernel covered with a thin powdery skin.

For 8 other species, see Eng. Bot.

\section{URCEOLARIA.}

1 Crust smooth, a little cracked, pale brick-coloured: shields reddish. Lichen. Eng. Bot.

For 5 other species, see Eng. Bot.

\section{LECANORA.}

1 Crust granulated, whitish: recept. flattish, flesh-coloured, with an elevated white border inflexed, at length waved. Lichen, Eng. Bot. For 45 other species, see Eng. Bot.

\section{PARMELIA.}

1 Thallus rigid, glaucous green, bearing dark green tufted excrescences; tawny and downy beneath; the lobes waved and laciniated. Recept. reddish-brown, margin wrinkled. Lichen. Eng. Bot. For 36 other species, see Eng. Bot.

\section{BORRERA.}

1 Thallus greenish glaucous grey; 'seg. linear, jagged, fringed, channelled beneath: recept. elevated, brownish black. Licheu. Eng. Bot.

$$
\text { For } 6 \text { other species, see Eng. Bot. }
$$

\section{CETRARIA.}

1 Thallus olive-brown, paler beneath; segments multifid, channelled, smooth, dentate-ciliate: fertile branches spreading : recept. flat, appressed; inargin elevated, entire. Lichen. Eng. Bot.

For 4 other species, see Eng. Bot. 
CLASS XXIV. CRYPTOGAMIA. ORDER V. LICHENES.

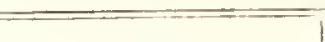

LINNEAN NAMES.

S'TIC'TA.

I parilis
I pulmonacea.....
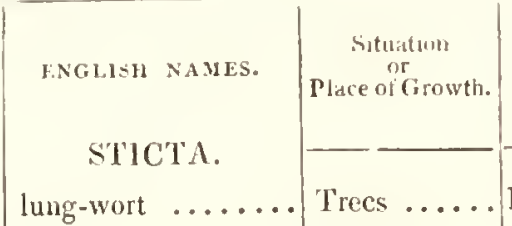

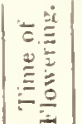

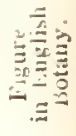

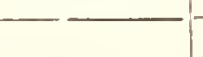

Trees .....1-12, 572

PELTIDEA.

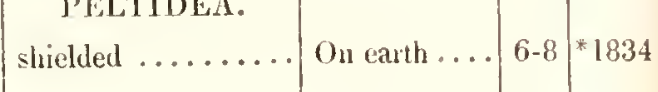

1 scutata.........

PELIIDEA.

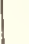

1

NEPHROMA. chocolatc.

Stoncs..... I-12 12360

ORCHALL. dyer's.

Mariti. rocks! $1-12=211$

EVERNII.

1 prunastri........

Stag's 1Iorn

Heath: $\ldots(1-12) * 859$

CINONOM CE.

l endivixfolia...... endive-leaved ......

1) ry places . . 11 - 202361

13 HOMYCES. pink 
CLASS XXIT. CRYPTOGAMHA. ORDER V. LICHENES.

SIFCIFIC CIIARACTER.

610. STICIA.

I Frond olive-green, cellular, reticulated; lobes laciniated, abrupt : recept. mostly marginal, bright chesnut; margin wrinkled. Lichen. Eng. Bot.

For 6 other species, see Eng. But.

\section{PELTIDEA.}

1 Frond sprcading, brownish grey, beneath pale, veiny: lobes oblong, with crisped and powdery edges: recept. asccnding, nearly flat, dark brown. Lichen. Eng. Bot.

Fon 8 other species, see Loudon's Eucy. Pl.

\section{NEPHROMA.}

1 Thallus lobed, livid brown, beneath naked, blackish: reept. brown. Lichen. Eng. Bot.

For another species, see Eng. Bot. 305.

\section{ROCCELLA.}

I Shrubby, solid, cylind. glaucous, somewhat branched, Iather ercet: recept. scattered, elevated; disk flat, as broad as the border. Licheu Roccella. Eng. Bot.

For unother species, see Eng. But. 728.

\section{EVERNIA.}

I Thallus grecnish white, leafy, much branched, pitted and rugged, very white beneath : recept. with a bright brown disk. Lichen. Eng. Bot.

\section{CENOMYCE.}

1 'Thallus large, decply lobed : seg. multifid, waved, crenate, crisped, yellowish grcen above, white beneatls: recept. simple, marginal, reddish brown. Lichen. Eng. Bot.

Fon 19 other species, see Loudon's Ency. I'l.

\section{BA:ONYCES.}

I Crust uniform, warty, whtish, with short cylind. stalks: recept. fleshcoloured. Bichen Baconyeres, liner. Bat. lor 3 other species, see ling. But. 
CLASS XXII. CRYPTOGAMI. ORDER I. LICHENES.

\begin{tabular}{|c|c|c|c|c|}
\hline LINNEAN SAMEA & LNGLISH NAMCS. & $\begin{array}{c}\text { situatyon } \\
\text { or } \\
\text { place of (irowth. }\end{array}$ & 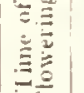 & 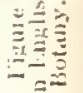 \\
\hline $\begin{array}{c}\text { Isllutul. } \\
\text { l corallinum ... }\end{array}$ & $\begin{array}{l}\text { INIDIUM. } \\
\text { white coral crusted. }\end{array}$ & & & \\
\hline
\end{tabular}

STEREOCAULON. STERLOCAULON.

1 paschale ....... crisp .........

Mic. rocks.. I - $12 * 282$

619

SPHARUPHORON. SPH AROPHORON.

1 coralloirdes

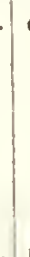

coralloid.

Rocks.....1-12*115

1 jubata

ALECTORIA.

ALECTORIA. rock hair.

Fir-trees.... $11-2 * 1880$ 


\section{1SIDIU11.}

1 Crust tartareons, whitish, papillary, uneven, with a leafy grey margin: recept. browuisls. Lichen. Eug. Bot.

For 4 other species, see ling. But.

\section{STEREOCAULON.}

1 'Thallus greenish grey, with numerous crowded brancles, and rongh with granulated excrescences: recept. scattered, terminal, dark brown. Lichen. Emar. But.

\section{SPIIEROPHORON.}

1 'Thallus yellowisl grcy; branches cylind. elongatcd, livaricating: recept. smooth. Lichen. Ling. Bot.

For 2 other species, see Eng. Bot. 114. 2474.

\section{THIBE III. HOMOTHALAMI.}

\section{ALECTORIA.}

1 Thallus shining, livid brown, very much branched; branches filiform, compressed at their divarications, pendulous or in tufts: recept. dark, with an entire border. Lichen. Jug. Bot.

Fine anuther species, see Eng. Bot. 2040.

\section{RAMILINA.}

1 'Thallus flat, linear, laciniated, greyish white, smooth, but wrinkled and pitted: ultimatebranches attenuated: reccpt.mostlymarginal, flat, pale fiesh-coloured. Lichen. Encr. Bot.

Fin 4 uther species, see Eng. Bot.

\section{CORNICULARIA.}

I 'Thallus somewhat compressed, smooth, blackish, disticliously dichotomous: branches fastigiate, black above: recept. flattish. blackish, marginatcd and toothed. Lichen. Fing. Bot.

fir 4 nther species, see Eng. Bot.

\section{USNFA.}

I Frond much branched. crect, grcenislı grey; branches sprearling, imple: recept. very broad, radiated. Jishen. Eug. Bot.

For 2 other species, sre ling. Bot. 
CLASS XXIY. CRYPTOGAMIA. ORDER V. LICIILNLS.

\begin{tabular}{|c|c|c|c|c|}
\hline WINNEAN NAMES. & ENGLISII NAMES. & $\begin{array}{c}\text { Situation } \\
\text { of } \\
\text { Place of Growth. }\end{array}$ & 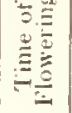 & 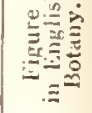 \\
\hline 1 nigrum.......... & blaek ........... & Cale. roeks. . & $\ldots$ & $* 1161$ \\
\hline 2 tenax $\ldots \ldots$ & tough ........ & Moist plaees & $\cdots$ & 2349 \\
\hline 3 palmatum ... & palmated......... & Sandy soil . & $5-8$ & *1635 \\
\hline 4 Burgessii ....... & Burgess's ...... & Trees ... & $1-12$ & $* 300$ \\
\hline 5 nigreseens $\ldots \ldots$ & blackish .......... & Trees .... & $1-12$ & $* 345$ \\
\hline 6 lacerum $\ldots . .$. & lacerated.......... & On earth .... & $3-5$ & *1982 \\
\hline $\begin{array}{c}\text { LEPRAIilA. } \\
1 \text { ochraeea ........ }\end{array}$ & $\begin{array}{l}\text { LEPRARIA. } \\
\text { oehre-coloured ... }\end{array}$ & Old trees. & $11-2$ & 2408 \\
\hline $\begin{array}{l}\text { OPEGRAPIIA. } \\
\text { I nimbosa } \ldots . . .\end{array}$ & $\begin{array}{l}\text { OPEGRAP'LA. } \\
\text { cloudy........... }\end{array}$ & Uld tices... & $1-12$ & 2346 \\
\hline $\begin{array}{l}\text { VERRUCARIA. } \\
\text { I punctiformis...... }\end{array}$ & $\begin{array}{l}\text { VERRUCARLA. } \\
\text { dot-striped ........ }\end{array}$ & Ash trees & $1-12$ & $* 2412$ \\
\hline $\begin{array}{l}\text { politna. } \\
1 \text { perlusil...... }\end{array}$ & $\begin{array}{r}\text { PORINA. } \\
\text { pienced } \ldots . . .\end{array}$ & linush of $\mathrm{tr}$. & 810 & $\cdot 677$ \\
\hline
\end{tabular}




\section{COLLEMA.}

1 Crust black; lobes of the circumferenee cut, crenate; ccntraI granular, a little branched: recept. black-cdged, finally convex. Licheu. Eng. Bot.

2 Imbricated: lobes thick, flattish, somewhat palmate: recept. depressed, with an entire border, reddish.

3 Thallus greenish brown, with distinct thick turgid lobes, somcwhat palmatc: reeept. reddish. Lichen. Eng. Bot.

4 Frond leafy, green, somewhat imbrieated, with round sinuated eurlcd lobes, woolly beneath: recept. brown, flattish, with a eurlcd border. Lichen. Eng. Bot.

5 Frond leafy, with broad membranous, blistered, round lobes, dull green: reeept. clustered, reddish brown. Lichen. Eug. Bot.

6 Frond leafy, menbranous, very thin, blueish brown; lobes dilated, finely jagged and fringed: reeept. scattcred, concave, red, with a pale border. Lichen. Eng. Bot.

TriBE IV. ATHALAMI.

\section{LEPRARIA.}

1 Crust not diseernible: fruetifieation of an oelirey-yellow, eollected into thin scattered patclies.

For 3 other species, see Eng. Bot.

\section{'Tribe V. PSEUDO-LICIIENES.}

\section{OPEGRAPIIA.}

1 Crust eraekcd, unequal, very whitc: reccpt. elustercd, minute, oblong, turgid; disk closed.

For 9 other species, see Loudm's lincy. I'l.

\section{VERRUCARIA.}

1 Crust thin, polislıcd, brown: recept. minute, without orifices: kerncl white. Jichen. Eng. Bot.

For 10 other species, see Loudon's Ency. I'.

\section{JURINA.}

1 C'mbt smooth, gleyish white: iccept. waxy, flesh-colourcd, with blath orifices. lichen. ling. Bot. 


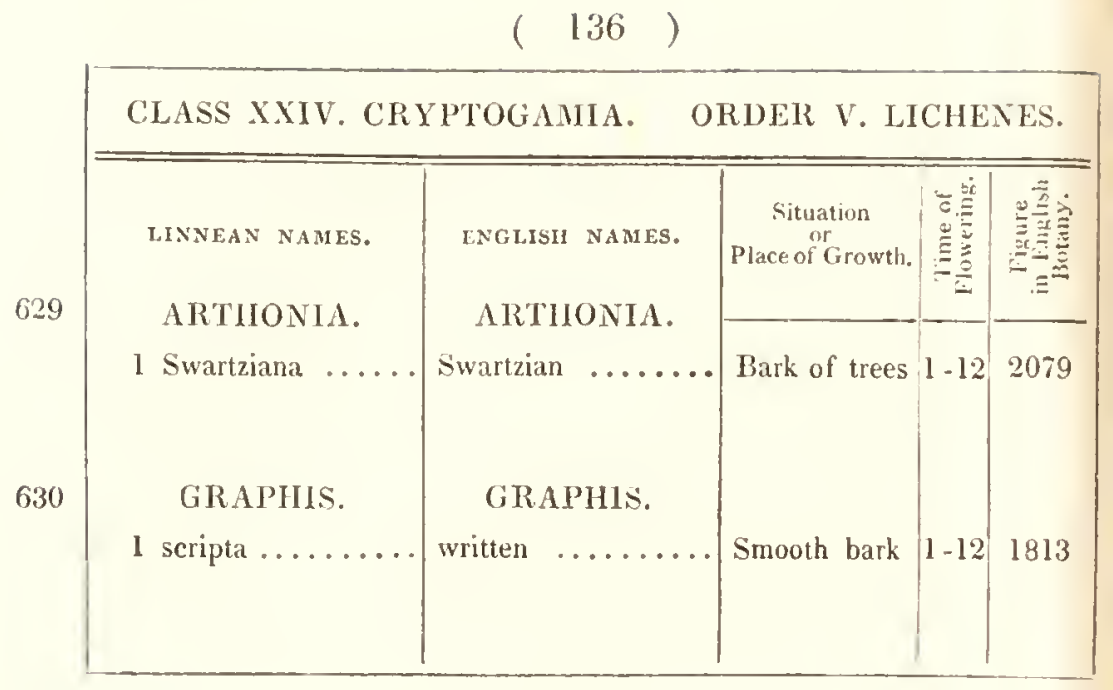


SPECIFIC CHARACTER.

\section{ARTHONIA.}

I Crust thin, membranous, scaly, cracked, cream-coloured: recept. black, depressed, roundish, wavy, rather uneven.

For 4 ither species, see Eng. Bot.

630. GRAPHIS.

1 Crust somewhat shining, greyish, bordered with black : recept. simple or branched; disk narrow.

For 4 other species, see Eng. Bot. 


\section{Subdivision 1. Mitrati.}

Recept. never closed. Hymenimm neither nargined nor discoid.

633 If on each surface, deflexed at the sides. Ilympnium occupying the whole outer surface.

634 Morchella.t Pilens lacunose, confluent with the stipes either at the margin or a little above it. Hymenium occupying the whole outer surface.

Subdivision 2. Cupmlati.

Receph. shaped like a reversed hell; equal. Ilymenium discivd, when yomng someuhat closed, smrounded hy the margin of the receptacle.

635 Prziza. Pilens mostly feshy, sessile or stipitate, more or less cup. shaped, sometimes becoming flat, when old.

\section{Division 3. Tremellini.}

Hymenimn confounded with a gelatimous reseplacle. Sporidia separate. Asci none.

636 Trenem.a. Recept. fructifying in all directions: without papillae. Sporidia nearly emerging.

\section{Division 4. Sicherotint.}

IHmenium confounded both with the fleshy receptacle, and the sporidio. Asce nome.

637 Scefnoruum. Subglobose, or without reyular form within, homogeneous, vesiculose, flesliy, or horny. Seeds nut known.

\section{Tubl II. GASTLROMVCETES.}

Fungus cutirely closed, and bearing sporidia in the rentre, thas forming an nterus.

\section{Division 1. ING1OCAstrLs.}

Iterns finally bursting forlh, apart from the receptacle. Aproriblu loded in the receptacle.

- Some of the species are used for purposes of cookery; nore especially the ll. esculenta, which is frequently substifuted for llie Morel.

+ liew of the fungi are so precious in the eyes of the epare as the fiatahle Morel. il. esculentid. "The lireach, however, are possessed of at species nu yet higher estimation, i. deliciosa. 


\section{Siubdivision 1. Phalloidere}

liecept. open bu the bursting of the utcrus. Sporidia in a mucous heyer. 638 P111.1.us. Ctipes issuing from a coliu. Pileus fumished with large eells filled with a seed-bearing slime.

Sublivision 2. Tuheracer.

Humenimm often gruted wich reins. Sporidiu, at first, mulpy.

639 'Tubl. L'ternsmarbled with veins inside. Spridia stalked, scattered among the veins. Subtrrancous.

Subdivision 3. Nidularialect.

l'torus filled witl separate cupsules.

640 Niderara. Involurrum simple. Calsule lenticular, fleshy, with sporidia in lieaps in the middle.

\section{Subdivision 4. Carpoboli.}

\section{Capsule solitaru.}

641 Pruokous. Stipes or receptaele pellucid, watery. Involucmm a roundish vesicle, bursting elastieally, plaeed on the apex of the reeptacle.

Division 2. PYRENomycetrs.

Sporidia disposeri in asci, in regular rous.

Subdivision 1. Splacriacei.

lurolucrum closed, perforated by an orifice.

642 Sprizria. Recppt. 0. Spherules sessile on the surfaee, or slightly immersed.

\section{Subdivision 2. Cutisponei.}

Asci none. Sporidia enclosed in a little bug or cell.

643 Crtispora. Many eelled: cells membranous, united at the ends.

Subdivision 3. Phacidiacei.

Involucrum finally bursting, with an open disk. Asci erect, fiued. 644 Pricinum. Recept. 0. Involucre sessile, depressed, bursting from the centre towards the cireumferenee in several aeute segments.

- Of this genus is the well known subterraneous luxury; called the 'Trufle: 'l" cilyarium; of whicli dogs are usually employed as the caterers. 


\section{Subdivision 4. Nylomacei.}

Asei obsolete. Sporidia innote.

645 Xrıors. Black, horny. lnvolucra single, solitary and minute, or united and eonfluent; irregularly dehiseent.

Division 3. Tnrenospenмr.

Sprordia intermined with little worlly tufts.

Subdivision 1. Lycoperdinei.

Vherus of a determinate fignre; fleshy when young.

646 Lycoperdon. Envelones membranaceous, sealy, with warts or soft spines, bursting irregularly at the apex, and eontaining a mass of seeds and woolly tufts.

Subdivision 2. Trichocisti.

Uterus regutar, pulpy when young.

647 Tnielia. Minute, subglobose or irregular. Furelope single, membranaceous, bursting. Filuments involute, attached to the base, and expanding elastically.

\section{Subdivision 3. Fuliginoidei.}

Uterus shapeless, pulpy when young. Shmidin separnted by molly tufts. 648 Lrcociara. Emelope single, fragile, variously dehiscent. A few filuments mingled with the seeds.

\section{Subdivision 4. Liceoidei.}

$$
\text { lufts obsotete. }
$$

649 Lrefa. Enrelope sessile, fragile, enelosing a powdery mass of seeds unmixed with filaments.

\section{Division 4. Mueonomils.}

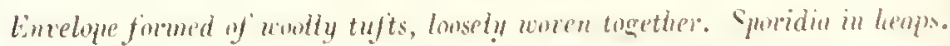
650 Mueon. linvelope stipitate, pellucil, at lcngth opaque: pediccl simple or branehed, tubular, articulated.

\section{Division 5. Penspoura.}

Envolne thin, somewhot membranons, bursting. Sporidia immersed. 651 Amplnsponiun. Subglobose. Seeds naked, of two forms. 


\section{Trire Jit. II Y PHOM Y CETES.}

Thullus tufled.

\section{Division l. Cephalotrichl.}

Recept. distinct, covered with wollu tofts, with sporidia scuttered among them.

652 Cerativa. Filaments short, pellueid, simple, attaehed to a membranous, plaited, simple or branehed, filiform reeptaele.

\section{Division 2. Strlborder.}

Fibres groun together upon the receptacle. Sporidia enchsed in a separate naked head.

653 Sirmbum. Minute: stipes slender, bearing a round solid head, whieh is at first pellueid and semi-fluid, finally more dense and opaque.

\section{Division 3. INoMreetes.}

Fibres genuine, somewhat separated by divisions. Recept. mone. Growing upou putrescent, arganic matter.

Subdivision 1. Byssacei.

Opaque fibres bearing seeds inside; when fertile, jointed; when sterile, contigums. Repelling moisture:

654 Torusa. Thallus composed of branehed, rigid, fragile, moniliform, somewhat opaque filaments; the articulations minute, globose.

Subdivision 2. Hucedines.

Tufts pellucid, with partitions, bearing seeds im the outside.

655 SEPEDON1Un. Thallus formed of entangled filaments, spreading within putrifying fungi. Sporidia seattered, globose, (bright yellow.)

\section{Division 4. PHYLLerineEA.}

Fihres spurious, contigums, beuring seeds inside. Recept. none. Groming on liviug leares.

656 Rupigo. Fibres funnel-shaped, or elub-shaped, twisted, situated in patelies on sickly leaves. 


\section{'T'RIRE IV. CONIOMICETES.}

Spmidia naker, withnt any heterogenenus receptacle.

Division 1. Tublencutant.F.

Sprevidin simple, srottered ater the receptacte.

657 Fustrium. Minute, subglobose, naked, almost entirely formed of fusiform, frec, jointless sporidia.

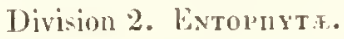

Sprridia separale, without a receptucle.

Subdivision 1. Stilbrsporei.

Grouing under the burk of dead plants.

658 Sulbospora. lilack. Recept. none, or a powdery mass intermixed with naked sporidia, the whole bursting through the bark.

Subdivision 2. Hypudermia.

Parasites upon living plunts.

659 Crlindroslorium. Very small; on the surface of living leaves. Sporidia pellucid, cylindrical, truncate, free, not divided. 


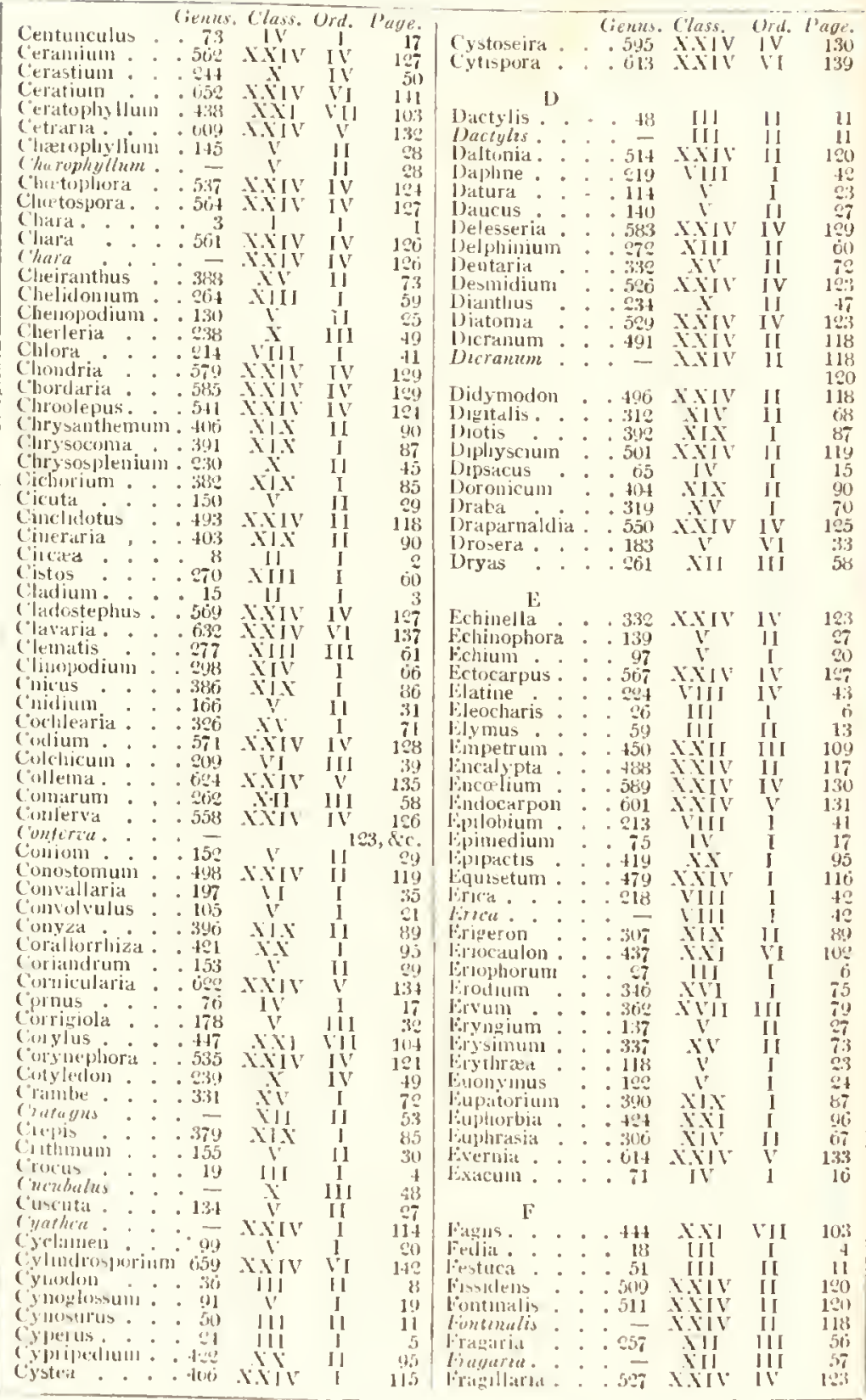


INDEX TO THE GENERA.

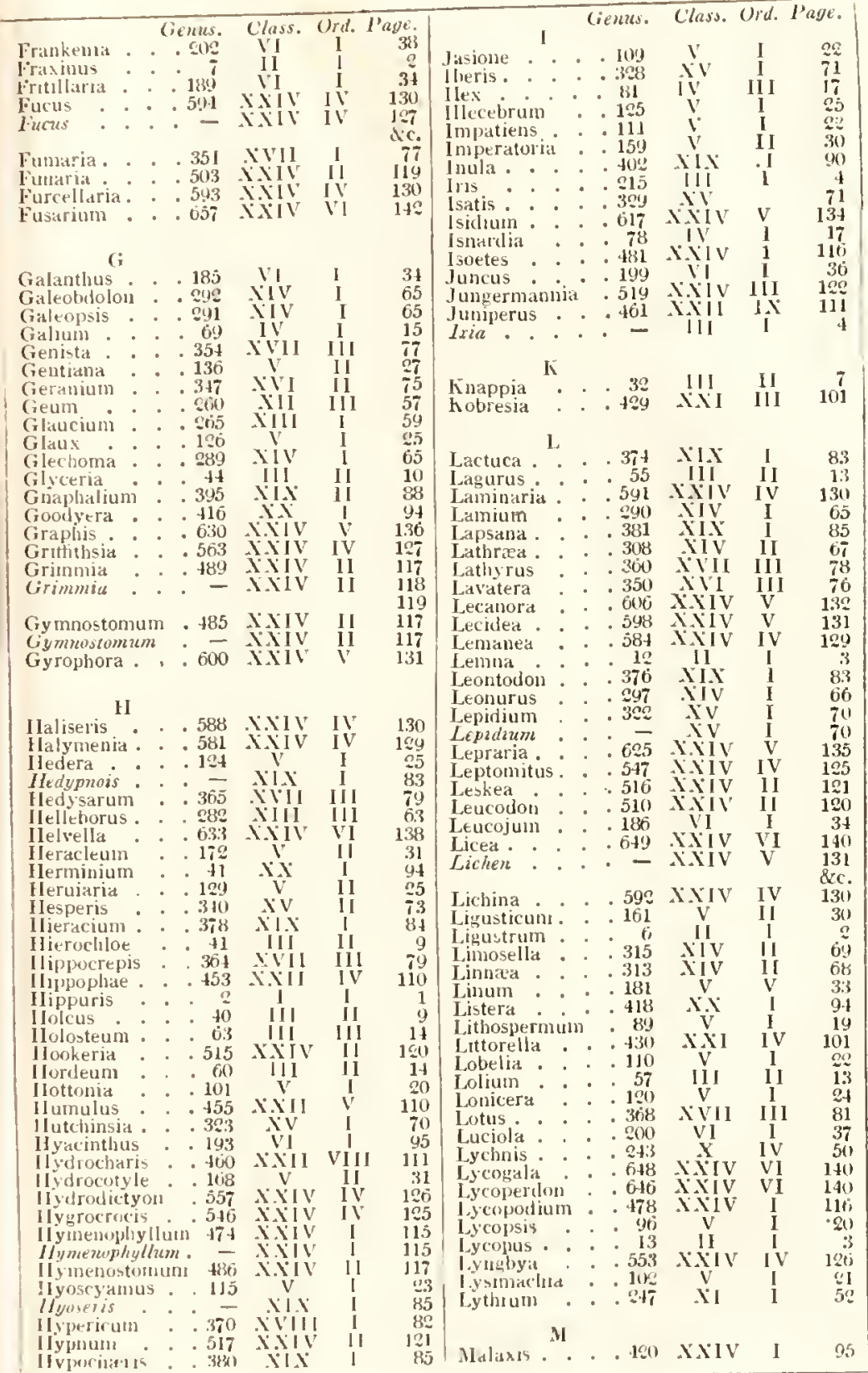


INDEX TO TJE GLIERA.

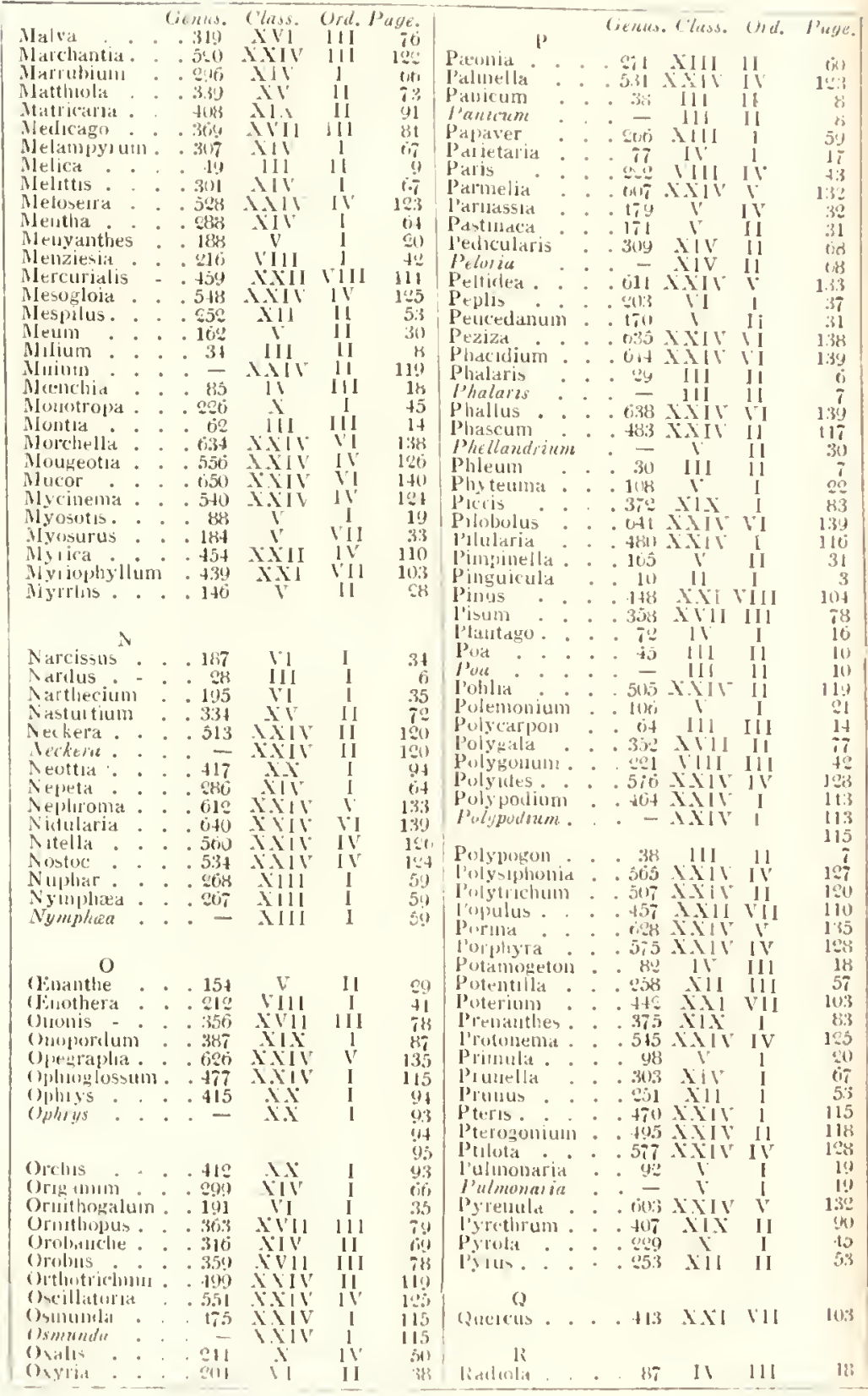




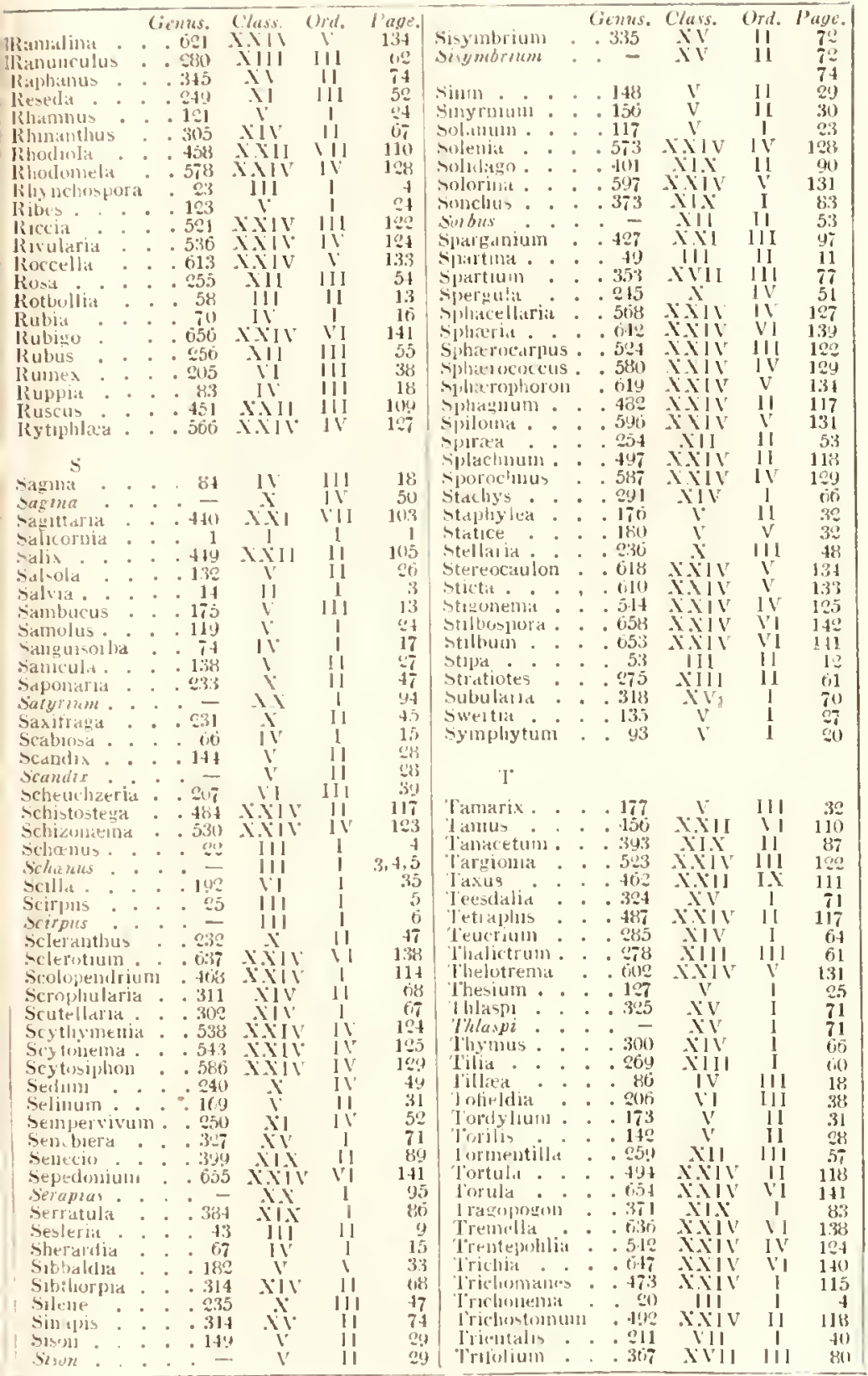


INDEX ()F THF GENERA.

\begin{tabular}{|c|c|c|c|c|c|c|c|c|c|c|c|c|}
\hline & & & Genus. & Class. & Ord. & l'aye. & & & Genus. & Class. & Ord. & Page. \\
\hline Triglochu & : & & .208 & 11 & II & 39 & Vellit & & * . 317 & $\mathbf{X V}$ & 1 & 70 \\
\hline Triticums & . & - & 61 & III & II & 10 & Verbent. & . & .113 & yiv & 1 & 23 \\
\hline Trollius. & . & . & .831 & XIII & JII & OQ & Veronica. & - & -201 & 11 & I & $t_{0}^{6 t}$ \\
\hline J'uber. & . & & .639 & XXIJ & 11 & 139 & Verrucaria & & .627 & Xiviv & V & 13.5 \\
\hline Julipa & - & - & . 190 & II & 1 & 34 & Glournum & . & .174 & v & [ [I] & 32 \\
\hline 'Jurritis. & - & - & . 342 & $X V$ & II & it & Vicia. . & . & .361 & XV11 & III & 78 \\
\hline Tussilago & - & . & . 398 & $X X$ & Iิ & 89 & Vinca. & . & .128 & V & I & 25 \\
\hline lyphat . & - & - & - 426 & AlI & III I & 47 & Viola. & . &.$\quad 112$ & $y$ & 1 & 28 \\
\hline & & & & & & & Viscum . & & . 452 & 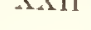 & 18 & 110 \\
\hline Ulex. & - & - & . 355 & IVII & II I & 77 & W & & & & & \\
\hline Ulmus. & & & .13 .3 & 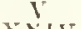 & 11 & $9 i$ & Weissia. & - & . +90 & IXIV & II & 118 \\
\hline Ulva. & & - & .574 & III & IV & 128 & Woodsia. & - & . . 178 & XNIV & I & 115 \\
\hline Urceolaria & . & . & .605 & XIIV & 1 & 130 & & & & & & \\
\hline Urtıcit & . & - & . 433 & XXI & IV & loge & Janthium & • & . . 434 & IXI & V & 102 \\
\hline I/ snea & & - & .023 & HEIV & $y$ & 134 & Aylomit. & - & . . 645 & X.YIS & 11 & $1+0$ \\
\hline Utricul & & & & & & & & & & & & \\
\hline V t it & & & & & & & Zannichelli & & •. . 425 & $\mathrm{XYI}$ & 1 & 90 \\
\hline Vaccinium & & - & - \&15 & $\operatorname{lin} 1$ & I & 41 & & - & . 590 & Arite & IV & 130 \\
\hline & - & - & - 17 & III & I & 4 & Kost & - & & & 1 & 1 \\
\hline & - & & . 604 & $x+1$ & $\mathrm{~V}$ & 132 & $2 y+1$ & - & - . 555 & XIIV & IV & 126 \\
\hline Vaucheria & - & & .570 & Axile & IV & 128 & Zygodon & . & . . 500 & XXI & II & 119 \\
\hline
\end{tabular}




\section{INDEX OF ENGLISH NAMES.}

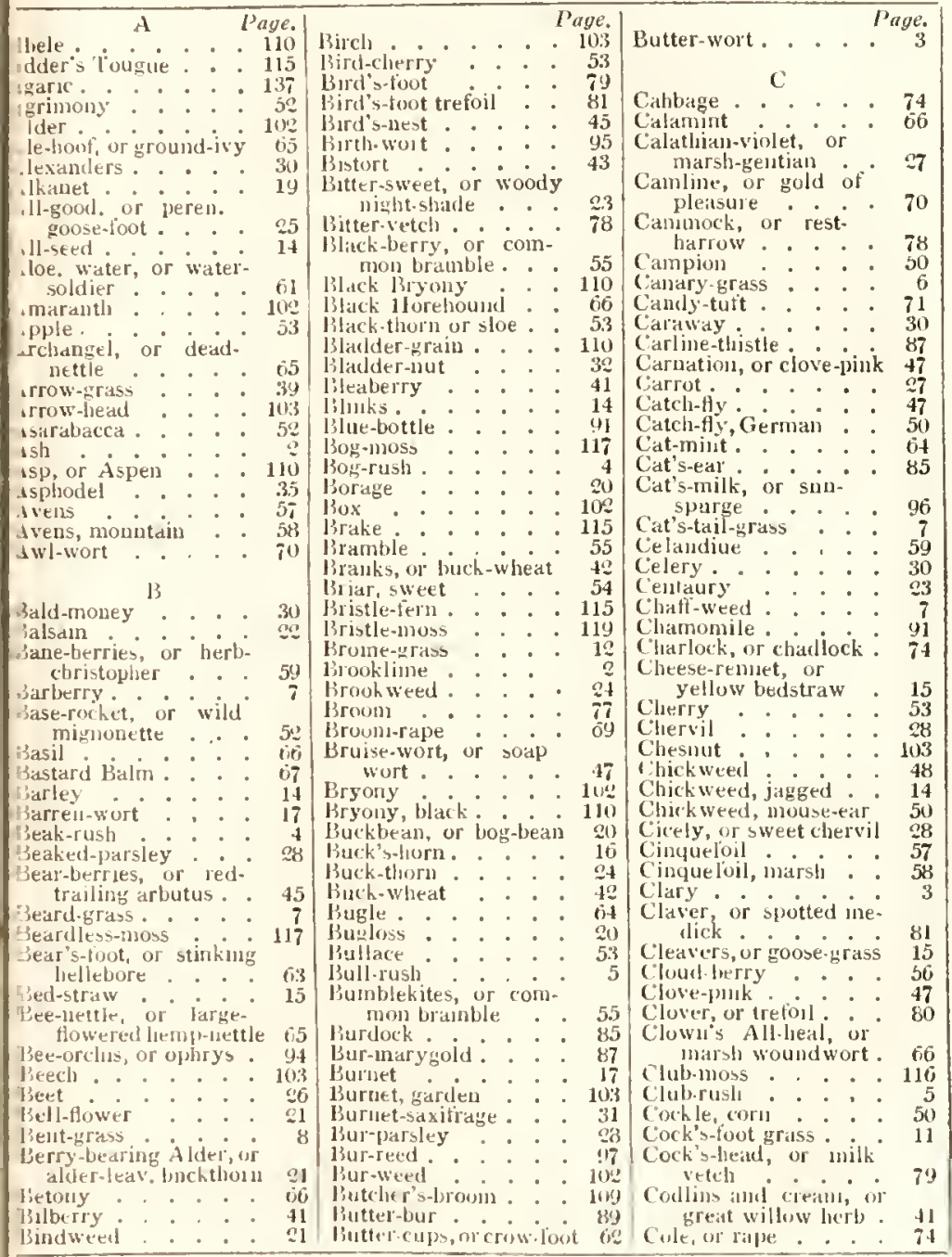




\section{INDEX OF ENGLISH NAMES.}

\begin{tabular}{|c|c|c|c|c|c|}
\hline & Page. & & & & \\
\hline r seit cabbils? & & Watch rusheses, or rongl & & (ilass-wort, or matsll- & \\
\hline 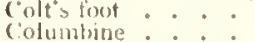 & $8 y$ & lorse-tail is & $11 \mathrm{fi}$ & situphlire . . & \\
\hline $\begin{array}{l}\text { Columbine : } \\
\text { Connfrey }\end{array}$ & iil & 1) wale, or derally night & & (ilote-Hluwer & $6 \%$ \\
\hline $\begin{array}{l}\text { Comfrey } \\
\text { Cone-moutied moss }\end{array}$ & * 50 & $\begin{array}{c}\text { slaide } \\
\text { Dyers-weed : }\end{array}$ & & $\begin{array}{l}\text { (iont w-beard } \\
\text { (ioliten-lod. }\end{array}$ & 8.3 \\
\hline $\begin{array}{l}\text { Cone-moutied moss } \\
\text { ('oral-ront. . . }\end{array}$ & $: \begin{array}{r}113 \\
95\end{array}$ & & & (ii)ldent-saxiliage: & 4.5 \\
\hline Coritl-wort . . & - $7 \%$ & E & & (iolel-ot-pleasure. & \\
\hline Cord-grass . . & il & liarthimoss . . . . & 117 & (joldy-locks - . & 87 \\
\hline $\begin{array}{l}\text { Corrlmoss : : } \\
\text { Cormander : }\end{array}$ & . 119 & 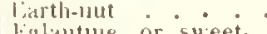 & 58 & Good hug lletury of & \\
\hline Cormalue-bottle: & gi & $\begin{array}{c}\text { Fongintue, or sweet. } \\
\text { brias. }\end{array}$ & & $\begin{array}{l}\text { peremot gilose-tuot } \\
\text { Gooseberry }\end{array}$ & $\begin{array}{l}25 \\
24\end{array}$ \\
\hline Conn-cochle. & 50 & Filde- . . . & 32 & Goose-foot: & 25 \\
\hline Cormel & 17 & lifectmpalue . & & liouse-grass... & 15 \\
\hline $\begin{array}{l}\text { money-wort } \\
\text { iryeold, ol yet }\end{array}$ & 68 & Elm $\quad \dot{0},{ }^{\prime}$ & & Guose-torgut, or sheed & \\
\hline $\begin{array}{l}\text { Irygold, ol yet } \\
\text { ox-tye }\end{array}$ & & Enchanter's nightshade & & wort ". . & \\
\hline $\begin{array}{l}\text { low ox-ye } \\
\text { conn-sillid. }\end{array}$ & $\begin{array}{c}90 \\
4\end{array}$ & Evenum primrose & & $\begin{array}{l}\text { forse, or furze } \\
\text { fout-weed }\end{array}$ & $\begin{array}{l}71 \\
3 i\end{array}$ \\
\hline & 6 & Everlisting . & & firang, alrow & 39 \\
\hline Cotton-thistle . & 67 & Herlastlly-peit. & & (ialass, barley & 14 \\
\hline Cotton-weed : & 87 & Extimingisluer moss & 117 & Grits, beard. & \\
\hline Couch-urass. & 14 & liye-briglt . . & & Gritss, beill. & \\
\hline Cow balle - & 29 & & & Girass, bronue & \\
\hline Cow-berry : & $\begin{array}{ll}11 \\
004\end{array}$ & Featluer-tuil, or water- & & Gritss, callaty ${ }^{\circ}$. & \\
\hline $\begin{array}{l}\text { arsieg, of cherei } \\
\text { arsuep }\end{array}$ & 83 & $\begin{array}{c}\text { Fether-full, or water- } \\
\text { vintet }\end{array}$ & & $\begin{array}{l}\text { (ilats, cat' tail. } \\
\text { (ilatss, coch, ticot: }\end{array}$ & \\
\hline $\begin{array}{l}\text { Cow-parsuep } \\
\text { Couslip }\end{array}$ & 20 & Featler-2rass & & 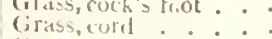 & 11 \\
\hline Cow-wheat: : & $i_{1}^{-}$ & leather-mo=s & & $\therefore$ cotton & \\
\hline Crato-apple . . . & 53 & felwort. & & (ilats, coucla or dong. & 14 \\
\hline Critke-belry, or crow & & lemuel . . . . & 30 & Girass, dog's tail: & 11 \\
\hline$y$. . . & $10 \%$ & lieru, comnnou, or blitke & 115 & 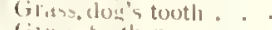 & \\
\hline (ranberiy. . & $4 !$ & liescuteglizss... & & 1ss.teather. & \\
\hline Cranesolibll. . & 75 & Fevertes & 90 & , tesc & 11 \\
\hline i ket. & .70 & fietel-midder. & & (i) its, huger. & \\
\hline & 73 & lig-wurt. . : & & (ili,(s), |ux-tat| & \\
\hline dit, or swines & 71 & Foluy ierng on lest' & 115 & (1) 193. gouse & \\
\hline r. . . & 70 & Finger-ntidss . . & & (ilass, lisule . & 13 \\
\hline. & 73 & is & & (i1,1se, hitir. & \\
\hline Cross-mort. & 15 & I. liag, sweel. & $3 i$ & lijaso, latale"s-tial & \\
\hline Crow-tont. & 62 & fllax $\cdot$. & & Glass, lueath . & 10 \\
\hline Ciow-garlick. . & 31 & lias-seed & & liram,lusly. & \\
\hline Conkow-flower, of rat & & fleit-bine & 8!! & (, riss, hust. & 49 \\
\hline 1 robun $\cdot$. . & 50 & Fleit-wort & (1) & Gritu, lyoue & 13 \\
\hline w-pint . & 30.3 & verd $\cdot$. B & & (rpiss, 11 ; & \\
\hline Curlbuar. & 132 & r-de-luce, or ills, & & (íits, meadow & \\
\hline Cutl-vecel & 87 & rims. $f+111$, or ()$_{5-}$ & & (irass, melic . & \\
\hline Cutrallt. & \& & $\mid$ |tul-yoy & 115 & Gilas, millet & \\
\hline () & & (III) (t) & & $\begin{array}{l}\text { (irils, luowor. } \\
\text { (ilass,uit }\end{array}$ & \\
\hline . . . . & 31 & Fonl's-parsley. & & Giras, prosuck: & \\
\hline & 90 & lorplet-me-not, or Water & & Giars,quiturug . & \\
\hline s-violet. & 73 & Mpull-grass. & 19 & (ilins, rye, or ditruel & 13 \\
\hline lefion . • . & 83 & Fork-1110) & & (irats, scorplou , . & \\
\hline 1)itne-wort, or dwall & & Finnt-touthed-moss & 117 & Gilats, worlyy. & il \\
\hline. & 32 & Fox-gluve . . & & (irass, sult . & \\
\hline or rye-naliss & $1: 3$ & -liail-ktasss. & & Girdss, sweet & \\
\hline intslidilt. & $2: 3$ & lirtillaty. & & rath, verditl & \\
\hline Dearlisetele. & 05 & liron-but. & 111 & Girass, wlieat & 11 \\
\hline 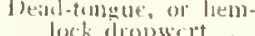 & & Finuitory . . . & $i z$ & (irass, whlutlow & (1) \\
\hline Devil's-bit? & 30 & lirze necille, ir yreet & & 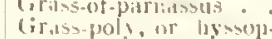 & \\
\hline & $5 b^{\circ}$ & wetd.... & 77 & .. leatred Is tarcuias. & \\
\hline Dittanuler, or pepper. & & & & firas-wrack . . & \\
\hline & ?! & $1 i$ & & Girendwed a & \\
\hline 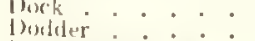 & $\begin{array}{l}3.3 \\
6 ;\end{array}$ & $\begin{array}{l}\text { Gintulugitle : } \\
\text { (iale. }\end{array}$ & 111 & 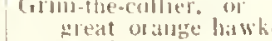 & \\
\hline on acis, or concis & it & Ciarclen-burnet: & 1018 & weed . . & \\
\hline -iail-fiass? & 11 & wk... & & (intuwetl . & 10! \\
\hline In grass, & 8 & & & (irallusd-ivy & \\
\hline d. ur corvel & 17 & (ientiantlid. & 10 & (1-ן)ाוc & \\
\hline & $-1 !$ & 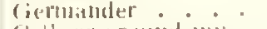 & & (iroubulsel & 89 \\
\hline t, watiol & 89 & (1) groulu4 $1 \mathrm{vy}$ & $(i, j)$ & Giuelikerelose - & \\
\hline & & ol'stinkilig & 1 & Gyjose. Wort, on "W. & \\
\hline [latele or white elesede] & 80 & 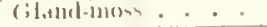 & Jis & & \\
\hline
\end{tabular}




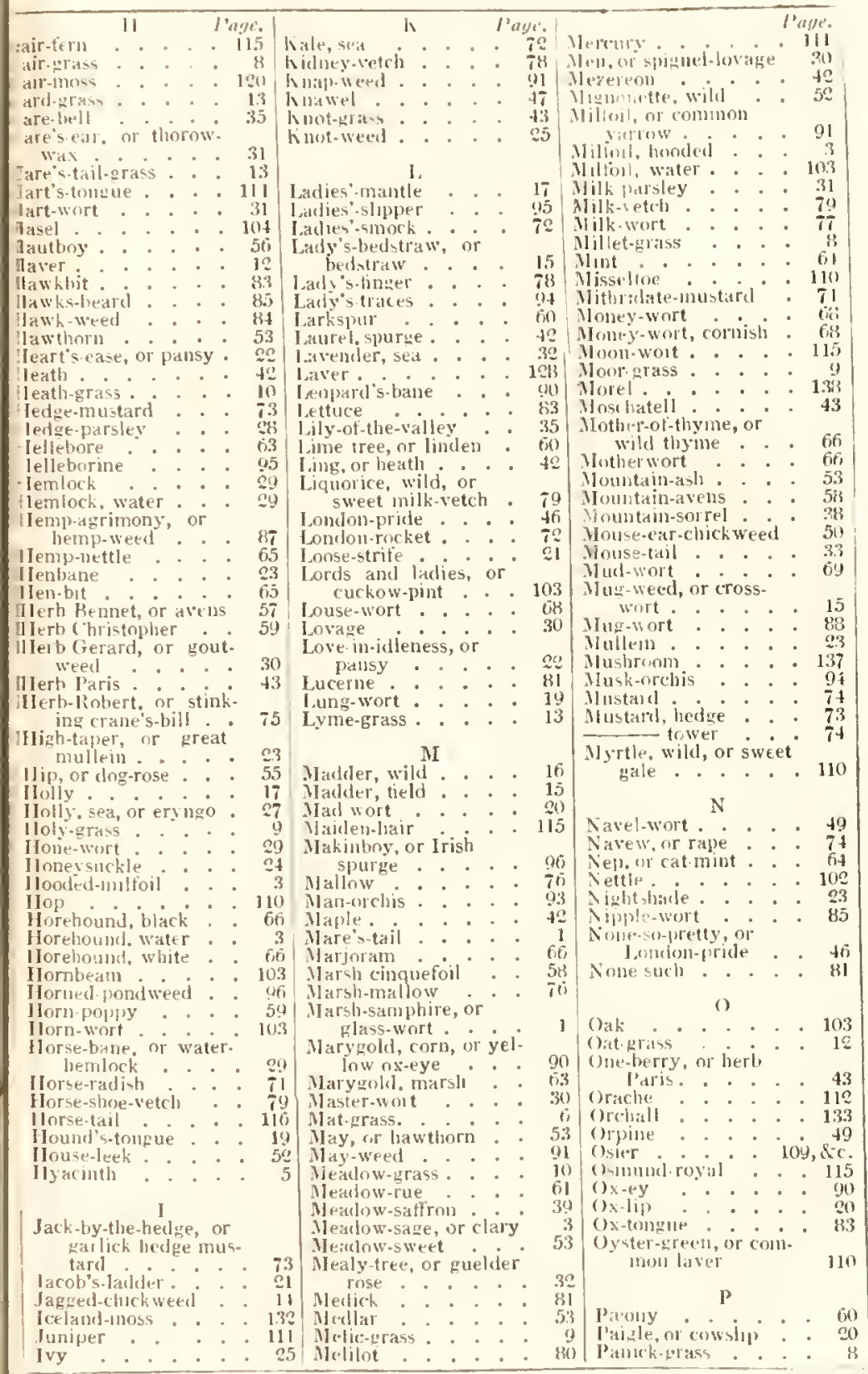




\begin{tabular}{|c|c|c|c|c|c|}
\hline & Prage. & & & & \\
\hline $\begin{array}{l}\text { Pansy } \\
\text { Park-leaves or tutsiu }\end{array}$ & & Rest-barrow & & Simson, or givundsel & \\
\hline Parnassus, grass of . & & $\begin{array}{l}\text { Rib-wort, or nob-grass } \\
\text { Roan-tree, or nounl. }\end{array}$ & & Skirret, or water pars. & \\
\hline Parsley, bur, or stone & & tain-ash & & skul1-cap & \\
\hline yolis: : & & Rock-cress, or Bristol & & Stoe $\cdot$ - & \\
\hline Parsley, milk. : & & Rock+t & & Smallage, or wild-celer & \\
\hline Parstev-piert, or field & & Rarket cress : : & & $\begin{array}{l}\text { Snake-weed : } \\
\text { Snap-lragon: }\end{array}$ & $\begin{array}{l}43 \\
60\end{array}$ \\
\hline mantle . & & Rocket, sed. . . & 71 & Sneeze-wort & \\
\hline $\begin{array}{l}\text { Parsuep } \\
\text { Parsnep co }\end{array}$ & & Rose $\cdot$. & 54 & Snow-cirop. & \\
\hline arsnep, cow & 31 & $\begin{array}{l}\text { Rose-ront } \\
\text { Rupture-wort: }\end{array}$ & 110 & Snow-flake . & 31 \\
\hline Pasque-flower. & 61 & Rush. : & $\frac{95}{36}$ & $\begin{array}{l}\text { Soalp-wort } \\
\text { Soft-s rass }\end{array}$ & 47 \\
\hline Pea.... & & IR usl, flowering & 44 & Soldier. water: & 61 \\
\hline Pear : • & & Rush, wood & 37 & Siolomou's seal & \\
\hline Pellitory-of-the-wall & & Rye-grass, or darnel & & Sorrel - borrel & $\begin{array}{l}38 \\
50\end{array}$ \\
\hline Penny-cress. & 71 & $\mathrm{~S}$ & & sow-hane, or redl goose & \\
\hline Penuy-royal . & 65 & Saffron $\cdot$ & 4 & toot i..... & \\
\hline per-saxitrage. & & Saffron, meadow & & Sow-bread . & 80 \\
\hline $\begin{array}{l}\text { wort : } \\
\text { kle. }\end{array}$ & 70 & Sage, meadow, or clar & & Sow-thistle. & 83 \\
\hline $\begin{array}{l}\text { Periwinkte } \\
\text { Persicaria : }\end{array}$ & & $\begin{array}{c}\text { Sa } \\
\text { der wood, or german }\end{array}$ & & $\begin{array}{l}\text { Spear-wort : } \\
\text { speedwell : }\end{array}$ & \\
\hline Petty-whin, or needle & & Saintfoin : : & & Speedwe or asparagus & \\
\hline veed · · & & Saint lobn's wort & & Sinder-orchis, or ophry & y4 \\
\hline Pig-nut, or earth-nut & $\begin{array}{l}62 \\
28\end{array}$ & $\begin{array}{l}\text { ter's wort, or } \\
\text { St. Jolnn's wo }\end{array}$ & & Spider-wort : : & \\
\hline Pile-wort . . . & 62 & . . . & $\cdot 108$ & Spikenard: & $\begin{array}{l}30 \\
89\end{array}$ \\
\hline Pill-wort & 116 & Sallow-thorn . & 110 & Spindie-tree & \\
\hline $\begin{array}{l}\text { Pimpernel } \\
\text { pimpernel, bastard, or }\end{array}$ & 21 & Silsafy * , & & Spleen-wort & 114 \\
\hline $\begin{array}{l}\text { Plmpernel, bastard, or } \\
\text { chatf-weed }\end{array}$ & 17 & Salt wort : & & $\begin{array}{c}\text { or scurvy- } \\
.\end{array}$ & \\
\hline Pine, or fir . . & 104 & Samphire : : & & Spuige : & \\
\hline Pink . * & 47 & Samplure, marsh, or & & Spurge-laurel. & \\
\hline Pipe-wort . . & & wlass-wort : & & Spur olive. & \\
\hline $\begin{array}{l}\text { Plantan } \\
\text { plantain, water : }\end{array}$ & 16 & Samphire, prickly & 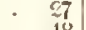 & Ypurrey . & \\
\hline $\begin{array}{l}\text { Plantain, water } \\
\text { Plowman's-spikenard }\end{array}$ & $\begin{array}{l}39 \\
: \quad 89\end{array}$ & Sand-wort $\cdot$ & 48 & Spurrey, sea & \\
\hline 3. & & Sauce-alone, or garlich & & Squinaticy-ivort or & \\
\hline Plume-thistle. & 86 & hed ze-mustard. & 73 & small woodrutt & \\
\hline Potypory • • & 113 & Saw-wort . . & & Stag's-horn & 133 \\
\hline Pol & 18 & Sixi & & Star-of-Rethlehem & \\
\hline Pond-weed, horned . & 96 & Saxilrage, golden & 45 & Star-thistle. . & 92 \\
\hline Poplar : : : & 110 & Silieep's & & ort $\cdot \dot{x}^{\circ}$ & \\
\hline Poppy, horn : : & $\begin{array}{l}59 \\
59\end{array}$ & Scorpion-grass. & ig & $\begin{array}{c}\text { ar-wort, water, or } \\
\text { star-leaf }\end{array}$ & \\
\hline Prickly samplire . & 97 & Scoteh fir. & 104 & Stitch-wort. & \\
\hline Prim & 20 & rrass & & itock. & \\
\hline ose, evening : & 41 & $\begin{array}{l}\text { Sea-lieath } \\
\text { Seatkale. }\end{array}$ & & or iock. & \\
\hline $\begin{array}{l}\text { nse-peerless, 01" } \\
\text { le narcissus. }\end{array}$ & 34 & levider: & & or rock- & 115 \\
\hline Privet . • . . . & & Sea-rocket . & $\pi 1$ & Stone-parsley, or bur- & \\
\hline Purslane $\cdot$. . & 38 & Sea.spurrey & & parsley • • & \\
\hline & & Seggrain, or ratwort & & Strap-wort: & \\
\hline ng-grass : : & 11 & Selt-heal. . & & stratwberry. & \\
\hline Quicken, or mountain & & or golden & & Strawberry-tree & \\
\hline Ouil wart & & & & Succory . . & \\
\hline Suill-wort & 110 & Sept-foil, or tormentil & & Sulphur-wort & \\
\hline & & or rough & & Siweet-briar. & \\
\hline & 74 & se-tail & 160 & Swet-thag. & \\
\hline 1-robiu. & & Sheep's bit, or scabious & & Sweetgale. & \\
\hline ort $\cdot$ & 89 & Shepherd's needle, or & & Sweet-yrass. & \\
\hline ons, or broad. & & $\begin{array}{l}\text { needle chervil } \\
\text { Sheplerd's ourse }\end{array}$ & & Switse's-cress, or wart & \\
\hline ed garlick. & 34 & $\begin{array}{l}\text { Shepherats purse } \\
\text { Shepherd's statt, or }\end{array}$ & & Sycanture: : & \\
\hline : $:$ : & it & teasel . . . & & & \\
\hline iw & $\begin{array}{l}55 \\
67\end{array}$ & $\begin{array}{l}\text { Shield-fern. } \\
\text { Shore-weed }\end{array}$ & 113 & & \\
\hline s, or tullitoit & 18 & Silver-weed : : & 57 & T'atusy. & 87 \\
\hline . . . . & 1.3 & Simpler's joy, or ver. & & late. & \\
\hline Reed-mate . . . . & & valls . . . . & & Teasel & \\
\hline
\end{tabular}


INDEX OF ENGLISH NAMES.

\begin{tabular}{|c|c|c|c|c|c|}
\hline & Page. & & P'age. & Whing or furce & \\
\hline $\begin{array}{l}\text { ender-twas-batele. } \\
\text { histle : . }\end{array}$ & . $\quad 95$ & Violet witer: & & $\begin{array}{l}\text { Whin, or furze } \\
\text { White-beam }\end{array}$ & $\begin{array}{l}71 \\
-53\end{array}$ \\
\hline histle & - 816 & $\begin{array}{l}\text { Yiolet, water } \\
\text { Viper's.bugloss }\end{array}$ & & White-horehonind" & \\
\hline $\begin{array}{l}\text { horn-apple }: \text { : } \\
\text { hrift - bort or water. }\end{array}$ & 38 & Vircins & & White-met, ol permy. & \\
\hline $\begin{array}{l}\text { hlum-wort, or water. } \\
\text { plantam }\end{array}$ & & iraveller's-joy & & White-thorm, or haw: & 31 \\
\hline 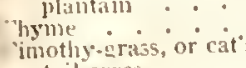 & 66 & $\begin{array}{c}\text { W } \\
\text { Wake-robin, or cuckow }\end{array}$ & & $\begin{array}{l}\text { thorn } \\
\text { White-tonth }\end{array}$ & \\
\hline $\begin{array}{l}\text { inothy-grass, or cat } \\
\text { tail-grass : : : } \\
\text { Toad-fitx : }\end{array}$ & s & Wake.robm, or cucnum & 10.3 & Whitlow-grass - & 70 \\
\hline $\begin{array}{l}\text { Toad-hiax, bistiri } \\
\text { load-flax, bets }\end{array}$ & 68 & Wall.cress. & & Whortle, or blea-berr & \\
\hline $\begin{array}{l}\text { Toad-flax, bastard } \\
\text { load-grass, or buttoin }\end{array}$ & 95 & Wall-thwer & & Willow bilow & 105 \\
\hline $\begin{array}{l}\text { l'oad-grass, or butfout } \\
\text { looth-wort. . . . }\end{array}$ & 17 & Wall-lettuce . - & $\begin{array}{r}83 \\
114\end{array}$ & $\begin{array}{l}\text { Willow-herb : } \\
\text { Winter-cress : }\end{array}$ & \\
\hline looth-wort : : & $\begin{array}{l}67 \\
57\end{array}$ & $\begin{array}{l}\text { Wallitue, ir yellow } \\
\text { Water-can, }\end{array}$ & & $\begin{array}{l}\text { Winter-cress } \\
\text { Winter-green : }\end{array}$ & \\
\hline $\begin{array}{c}\text { Tormentil } \\
\text { Tow balsand, or yei. }\end{array}$ & & water-fily?. & & Woad & \\
\hline $\begin{array}{l}\text { low balsam : } \\
\text { Tower-mustard : }\end{array}$ & 2 & $\begin{array}{l}\text { Water-cress } \\
\text { Water-drop-wort }\end{array}$ & $7 \cong$ & & 61 \\
\hline $\begin{array}{l}\text { Tower-mustard } \\
\text { I'raveller's.joy }\end{array}$ & 61 & $\begin{array}{l}\text { Water-drop-wort } \\
\text { Water-lomlock }\end{array}$ & 29 & $\begin{array}{l}\text { Woodbine, or honcy- } \\
\text { suckle }\end{array}$ & \\
\hline $\begin{array}{l}\text { Iraveller s-joy } \\
\text { Iretoil }\end{array}$ & $\begin{array}{l}01 \\
80\end{array}$ & Water-horehound & 3 & Woodruft" & $\begin{array}{l}24 \\
15\end{array}$ \\
\hline Iree-mallow herb $\mathrm{Par}$ & 70 & Water-lily: . & & Wond-rusít . . & 37 \\
\hline $\begin{array}{l}\text { lrue-love, or herb Par } \\
\text { liruttee }\end{array}$ & 43 & Water-mitioil. & 103 & Wood-sage, or germa & \\
\hline $\begin{array}{l}\text { lruttle : : : : } \\
\text { liulip }: \text { : }\end{array}$ & 139 & Water-moss & 190 & der & $\begin{array}{l}64 \\
50\end{array}$ \\
\hline lulip : : : & 31 & $\begin{array}{l}\text { Water-parsmep : } \\
\text { Water-plantain : }\end{array}$ & 39 & Wormwood.: & 88 \\
\hline Turmp : : : & 82 & Water-soldier, or aloe & & Wound-wort . & \\
\hline Tway-blade : & 94 & Water-star-leaf, or & & Wycli-eln . & 26 \\
\hline Twig-rush $\cdot$. & . & $\begin{array}{l}\text { star-wort : : } \\
\text { Water-violet : }\end{array}$ & & $\mathrm{Y}$ & \\
\hline $\mathrm{v}$ & & Water-wort. & & Farrow & \\
\hline Valerian $\cdot{ }^{\circ}$ & 4 & Wart-cress & 71 & Yellow-rattle & $\begin{array}{l}67 \\
59\end{array}$ \\
\hline $\begin{array}{l}\text { Valerian, Greek, or } \\
\text { Jacob's-ladder }\end{array}$ & & Wart-wort, or sun- & & Yellow-weed, or dye & \\
\hline Vernal-grass & 3 & Waytaring-tree, or & & weed & 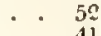 \\
\hline Vervain. . & 64 & inealy guelder-ros & 32 & Yellow-wort & \\
\hline 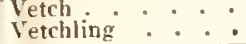 & $\begin{array}{r}79 \\
78\end{array}$ & $\begin{array}{l}\text { Weasel-snout : : } \\
\text { Wheat-grass }\end{array}$ & $\begin{array}{l}65 \\
14\end{array}$ & Yew. - & \\
\hline
\end{tabular}




\section{INDEX TO THE NATURAI, ORDERS.}

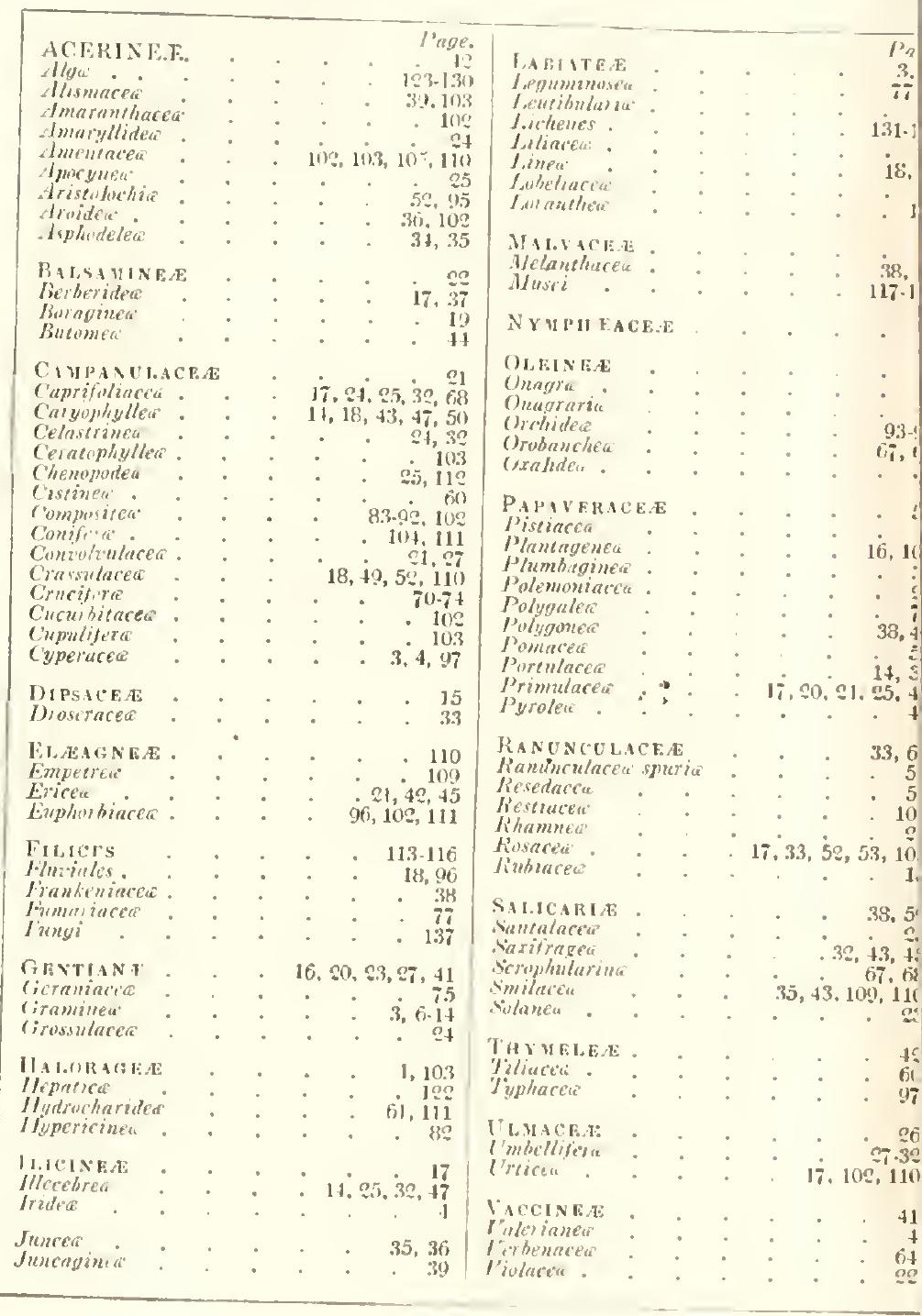





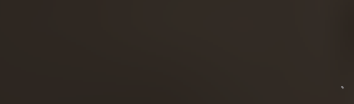

b
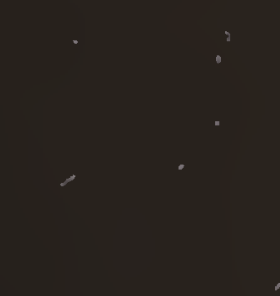
